DEDUCTIVE SYSTEMS AND THE DECIDABILITY PROBLEM FOR HYBRID LOGICS 
蓄 


\section{DEDUCTIVE SYSTEMS AND THE DECIDABILITY PROBLEM FOR}

HYBRID LOGICS

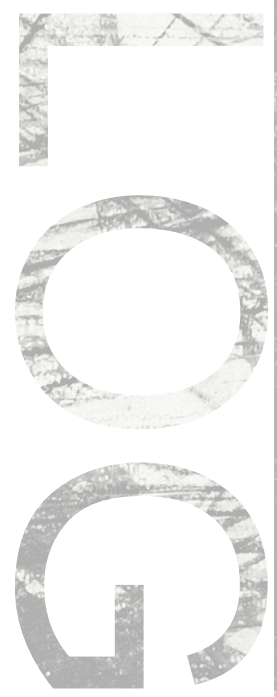

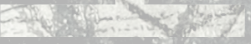
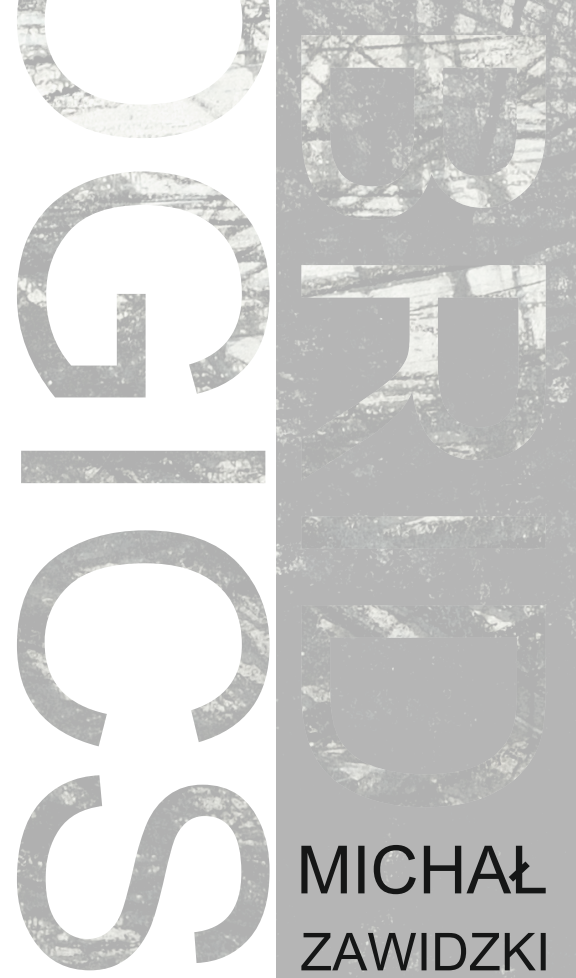

MICHAŁ ZAWIDZKI 
Michat Zawidzki - University of Łódź, Faculty of Philosophy and History

Department of Logic and Methodology of Sciences, 16/18 Kopcinskiego St., 90-232 Łódź

e-mail: michal.zawidzki@gmail.com

(C) Copyright by University of Łódź, Łódź 2014

(C) Copyright for this edition by Jagiellonian University Press

All rights reserved

No part of this book may be reprinted or utilised in any form or by any electronic, mechanical or other means, now known or hereafter invented, including photocopying and recording, or in any information storage or retrieval system, without permission in writing from the publishers

Published by Łódź University Press \& Jagiellonian University Press

First edition, Łódź-Kraków 2014

W.06463.14.0.D

ISBN 978-83-7969-042-8 paperback Łódź University Press

ISBN 978-83-233-3695-2 paperback Jagiellonian University Press

ISBN 978-83-7969-043-5 hard cover kódź University Press

ISBN 978-83-7969-054-1 electronic version kódź University Press ISBN 978-83-233-9018-3 electronic version Jagiellonian University Press

Łódź University Press

8 Lindleya St., 90-131 Łódź

www.wydawnictwo.uni.lodz.pl

e-mail: ksiegarnia@uni.lodz.pl

phone (42) 66558 63, fax (42) 6655862

WYDAWNICTWO
UNIWERSYTETU
ŁÓDZKIEGO

Distribution outside Poland

Jagiellonian University Press

9/2 Michałowskiego St., 31-126 Kraków

phone +48 (12) 63101 97, +48 (12) 66323 81, fax +48 (12) 6632383

cell phone: +48 506006 674, e-mail: sprzedaz@wuj.pl

Bank: PEKAO SA, IBAN PL 80124047221111000048563325

www.wuj.pl

$\chi \frac{\text { JAGIELLONIAN }}{\text { UNIVERSITY PRESS }}$ 
List of Figures viii

List of Tables $\quad \mathrm{x}$

Abbreviations $\quad$ xi

Symbols $\quad$ xii

Acknowledgements xiii

1 Introduction 1

I Logical Preliminaries - Hybrid Logics, Decidability, Deductive Systems $\quad 7$

2 Modal logic, decidability and complexity 9

2.1 Modal logic . . . . . . . . . . . . . . . . . . . . . . . . . 9

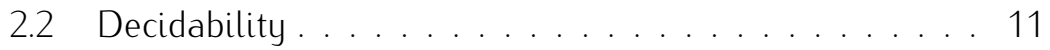

2.3 Complexity . . . . . . . . . . . . . . . . 12

3 Deductive systems 17

3.1 Sequent calculi . . . . . . . . . . . . . . . . . 18

3.2 Tableau calculi . . . . . . . . . . . . . . . . . . . . . 19

4 Hybrid logic 23

4.1 Motivation . . . . . . . . . . . . . . . . . . 23

4.2 Syntax and semantics ... . . . . . . . . . . . . 24

4.3 Expressivity... . . . . . . . . . . . . . . . . . . 27

4.3.1 Expressive power . . . . . . . . . . . . . . 27

4.3.2 Hierarchy of expressive power . . . . . . . . 29

4.4 Hilbert-style calculi . . . . . . . . . . . . . . . . . . . 30

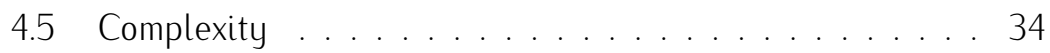


4.6 Historical overview . . . . . . . . . . . . . . . . 36

$5 \operatorname{Logic} \mathcal{M}\left(\mathrm{E}_{n}\right) \quad 39$

5.1 Motivation . . . . . . . . . . . . . . . . . . . . . 39

5.2 Syntax and semantics . . . . . . . . . . . . . . . . . 40

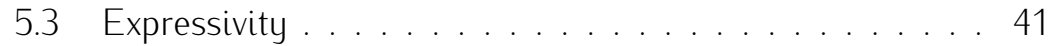

5.4 Hilbert-style calculus for $\mathcal{M}_{K}\left(E_{n}\right) \ldots \ldots \ldots . \ldots 42$

5.4.1 Axiomatization for $\mathcal{M}_{K}\left(E_{n}\right) \ldots \ldots . . . .442$

5.4 .2 Adequacy of $\mathcal{M}_{K}\left(E_{n}\right) \ldots \ldots \ldots 43$

5.5 Complexity . . . . . . . . . . . . . . . . . 57

5.6 Historical overview . . . . . . . . . . . . . . . . 84

6 Remarks on description logics contributions 87

II Deductive Systems for Hybrid Logics 91

7 Deductive systems for standard hybrid logics 93

7.1 Introduction to tableau calculi for the logic $\mathcal{H}_{\text {b-f }} \ldots \ldots 95$

7.2 Prefixed calculus for $\mathcal{H}_{\text {b-f }} \ldots \ldots \ldots \ldots$. . . . . 96

7.2.1 Meta-language for $\mathcal{H}_{\mathrm{b}-\mathrm{f}} \ldots \ldots \ldots \ldots 9 . \ldots 96$

7.2.2 Tableau calculus $\mathcal{T}_{\mathcal{H}_{\text {b-f }}}^{\mathrm{p}} \ldots \ldots \ldots . . . . . .99$

7.2.3 Soundness and completeness of $\mathcal{T}_{\mathcal{H}_{\mathrm{b}-\mathrm{f}}}^{\mathrm{p}} \ldots \ldots 103$

7.3 Rule refinement of $\mathcal{T}_{\mathcal{H}_{\mathrm{b}-\mathrm{f}}}^{\mathrm{p}} \ldots \ldots \ldots \ldots \ldots . \ldots \ldots$

7.4 Internalized calculus for $\mathcal{H}_{\mathrm{b}-\mathrm{f}} \ldots \ldots \ldots \ldots \ldots \ldots$

7.4.1 Encoding domain sort expressions ........ . 112

7.4.2 Tableau calculus $\mathcal{T}_{\mathcal{H}_{\text {b-f }}}^{\mathrm{i}} \ldots \ldots \ldots \ldots . \ldots . \ldots 113$

7.4.3 Decision procedure based on $\mathcal{T}_{\mathcal{H}_{\text {b-f }}}^{\mathrm{i}} \ldots \ldots . .115$

7.4.4 Soundness and Completeness of $\mathcal{T}_{\mathcal{H}_{\mathbf{b}-f}}^{i} \ldots \ldots 116$

7.5 Sequent Calculus for $\mathcal{H}_{\mathrm{b}-\mathrm{f}} \ldots \ldots \ldots \ldots \ldots$

7.6 Termination of $\mathcal{T}_{\mathcal{H}_{\mathrm{b}-\mathrm{f}}}^{\mathrm{i}} \ldots \ldots \ldots \ldots \ldots \ldots . \ldots \ldots$

7.7 Remarks on blocking mechanisms . . . . . . . . . 126

7.7.1 Subset blocking, equality blocking . . . . . . . 126

7.7.2 Pattern-based blocking . . . . . . . . . . . 132

7.8 Concluding remarks . . . . . . . . . . . . . . . 133 
8 Deductive systems for non-standard hybrid logics 135

8.1 Prefixed calculus for $\mathcal{M}\left(\mathrm{E}_{n}\right) \ldots \ldots . \ldots 136$

8.1.1 Meta-language for $\mathcal{M}\left(\mathrm{E}_{n}\right) \ldots \ldots . \ldots 136$

8.1.2 The tableau calculus $\mathcal{T}_{\mathcal{M}\left(\mathrm{E}_{n}\right)} \ldots \ldots$. . . . . . 137

8.1.3 Soundness and completeness of $\mathcal{T}_{\mathcal{M}\left(\mathrm{E}_{n}\right)}^{P} \ldots \ldots 138$

8.2 Termination of the calculus . . . . . . . . . . . . 141

8.3 Rule refinement of $\mathcal{T}_{\mathcal{M}\left(\mathrm{E}_{n}\right)} \ldots \ldots \ldots \ldots \ldots 146$

8.4 Internalized calculus for $\mathcal{M}\left(\mathrm{E}_{n}\right) \ldots \ldots . \ldots 147$

8.4.1 Encoding domain sort expressions . . . . . . . 147

8.5 MetTeL2 implementation . . . . . . . . . . . . . . . 151

8.6 Other existing decision procedures . . . . . . . . . . 152

8.6.1 Analytic tableau calculus with pattern-based blocking ...................... 152

8.6.2 Constructive tableau calculus with pairwise blocking ........................ 161

8.6.3 Constructive tableau calculus with arithmetic rea-

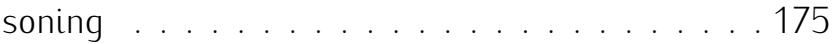

8.7 Concluding remarks . . . . . . . . . . . . . . . . . 191

9 Summary 195

9.1 Results . . . . . . . . . . . . . . . . . . . . . 195

9.2 Future research . . . . . . . . . . . . . . . . . 196

$\begin{array}{ll}\text { Bibliography } & 199\end{array}$

$\begin{array}{ll}\text { Index } & 211\end{array}$ 


\section{LIST OF FIGURES}

1 Hierarchy of hybrid languages with respect to their expressive power . . . . . . . . . . . . . . 30

2 The family $\bar{\Gamma}_{\varphi} \ldots \ldots \ldots \ldots \ldots \ldots \ldots$

3 Formulas that encode a grid and a tiling ........ 61

4 "Gluing together" states by Num . . . . . . . . . . . . 63

5 Formulas that encode a grid and a tiling over reflexive

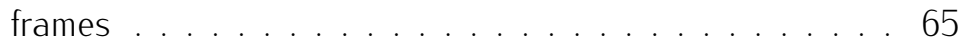

6 Formulas that encode a grid and a tiling over symmetric frames . . . . . . . . . . . . . . . . . . . 68 68

7 Formulas that encode a grid and a tiling over serial frames ... . . . . . . . . . . . . . . . . 71

8 Formulas that force a tree, the bottom "layer" of which will be arranged as a grid ... . . . . . . . . . . 74

9 Formulas that link up points from the $n$-th, bottom layer of the tree into a grid, and encode a tiling . . . . . . . 79

10 Semantic specification of connectives $S^{0}$ for the logic $\mathcal{H}_{\text {b-f }} \ldots \ldots \ldots \ldots$. . . . . . . . . . . . . 99

11 Sets $S_{+}^{0}$ and $S_{-}^{0}$ of Skolemized rules for connectives . . 100

12 Semantic specification of background theory $S^{b}$ for $\mathcal{H}_{\text {b-f }} 101$

13 The rules of $\mathcal{T}_{\mathcal{H}_{\text {b-f }}}^{\mathrm{p}} \ldots \ldots \ldots \ldots \ldots \ldots \ldots \ldots \ldots \ldots$

14 Refined rules of $\mathcal{T}_{\mathcal{H}_{\text {b-f }}^{\mathrm{p}}}^{\mathrm{p}} \ldots \ldots \ldots \ldots \ldots \ldots \ldots$

15 Derivation of the initial rule $(\neg D)$ using the refined rule $(\neg \mathrm{D})$ and $(\mathrm{ub}) \ldots \ldots \ldots \ldots . \ldots . \ldots \ldots$

16 Rules for the calculus $\mathcal{T}_{\mathcal{H}_{\text {b-f }}}^{\mathrm{i}} \ldots \ldots \ldots \ldots . \ldots 114$

17 Rules for the sequent calculus $\mathcal{S}_{\mathcal{H}_{\text {b-f }}} \ldots \ldots \ldots \ldots 118$

18 Various models for the formula $\mathrm{A}(\nabla \varphi) \ldots \ldots \ldots 121$

19 Formula $p \wedge \mathrm{A}\left(\diamond p \wedge \square^{-} \square^{-} \neg p\right)$ breaking the completeness of the calculus with with restriction $(R)$ for the

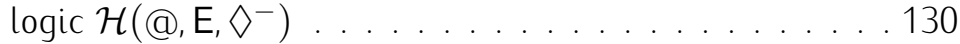


20 Semantic specification of connectives $S^{0}$ for the logic

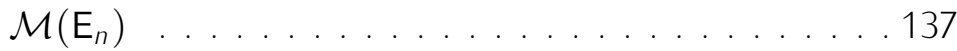

21 Sets $S_{+}^{0}$ and $S_{-}^{0}$ of Skolemised rules for connectives . . 137

22 Semantic specification of background theory $S^{b}$ for $\mathcal{M}\left(E_{n}\right) 138$

23 The rules of $\mathcal{T}_{\mathcal{M}\left(\mathrm{E}_{n}\right)}^{p} \ldots \ldots \ldots \ldots \ldots \ldots \ldots \ldots$

24 Formula $\mathrm{E}_{=0}\left(\mathrm{E}_{=0} \varphi\right)$ generating infinite $\mathcal{T}_{\mathrm{K}(\mathrm{E})_{n}}$-derivartion142

25 Hybrid translation function for domain-sort expressions 147

$26 \mathcal{M}\left(\mathrm{E}_{n}\right)$-translation function for domain-sort expressions 148

27 Rules for the calculus $\mathcal{T}_{\mathcal{M}\left(\mathrm{E}_{n}\right)}^{i} \ldots \ldots \ldots \ldots \ldots$

28 Rules for the calculus $\mathcal{T}_{\mathcal{S H O Q}} \ldots \ldots \ldots \ldots \ldots$

29 Models of a formula $\mathrm{E}(\diamond p)$ generated by $\mathcal{T}_{\mathcal{M}\left(\mathrm{E}_{n}\right)}^{p}$ and $\mathcal{T}_{\mathcal{S H O Q}} \ldots \ldots \ldots \ldots \ldots \ldots \ldots$

30 Step-by-step construction of a non-deterministic finite automaton . . . . . . . . . . . . . . . . . . 167

31 Rules of the constructive tableau calculus for the logic $\mathcal{M}\left(E_{n}\right)$, based on the calculus for the logic $\mathcal{S H I} \mathcal{Q} \ldots 171$

32 Explicit rules for frame conditions . . . . . . . . . . 174

33 Rules of the calculus for the logic $\mathcal{M}\left(\mathrm{E}_{n}\right)$, which involves an inequality solver . . . . . . . . . . . . 183 
1 Complexity classes for the logics $\mathcal{H}(@)$ and $\mathcal{H}(\mathrm{E})$ defined over various frame classes . . . . . . . . . . . 35

2 Complexity classes for the logic $\mathcal{M}\left(\mathrm{E}_{n}\right)$ defined over various frame classes . . . . . . . . . . . . . 83

3 Symbols denoting various elements of description logic

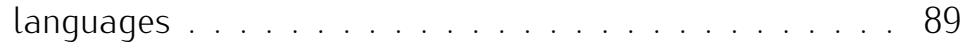




\section{ABBREVIATIONS}

TM Turing machine

$\operatorname{Card}(A)$ cardinality of a set $A$

CT classical tautologies

MP modus ponens

MCS maximal consistent set

$\log \quad$ logarithm to the base 2

NFA non-deterministic finite automaton

clos closure set of formulas

Sub set of subformulas

NNF negation-normal form 
$\mathcal{M}_{\mathrm{C}}(\mathrm{S})$ modal logic defined over a class of frames named $\mathrm{C}$, augmented with operators from a set $\mathrm{S}$

$\mathcal{H}_{C}(\mathrm{~S})$ hybrid logic defined over a class of frames named $\mathrm{C}$, augmented with operators from a set $\mathbf{S}$

$\mathfrak{M} \quad$ modal model

$\mathcal{B}$ branch of a tableau

$\mathcal{T}_{\mathcal{L}} \quad$ tableau calculus for a logic $\mathcal{L}$

$\mathcal{T}^{p} \quad$ prefixed tableau calculus

$\mathcal{T}^{i} \quad$ internalized tableau

$\mathcal{S}_{\mathcal{L}}$ sequent calculus for a logic $\mathcal{L}$

$\mathfrak{M}(\mathcal{B})$ modal model obtained from a branch $\mathcal{B}$

$\mathcal{P}(A)$ power set of a set $A$ 
The more he looked inside the more Piglet wasn't there.

- Alan A. Milne, "The house at Pooh Corner"

ACKNOWLEDGEMENTS

Deductive sciences have this strange property: sometimes the more you seek for a solution to a problem, the more it is not there. Logic is not an exception. That is why you need strong guidance on your scientific path. Otherwise, you can end up hopelessly going in circles. I had enough luck to meet the right people on my academic way.

Obviously, I would like to express my gratitude to all who have made even the tiniest contribution to this book which in its initial form was written as a PhD thesis, defended at the Faculty of Philosophy and History of the University of Lodz. Unfortunately, I will have to restrict myself to a finite number of people whose names I would like to mention, hoping that the rest of them will forgive me.

First and foremost, my gratefulness goes to my supervisor, Andrzej Indrzejczak, whose patience and kind insistence led me to the point at which I could say "Okay, I'm finished". I do not even want to think where I would be now but for him motivating and encouraging me to work. I could always count on him. In purely scientific terms, I am also in his debt for the observation concerning the behaviour of rules for the D operator in the calculus I previously devised.

I also owe words of gratitude to Renate Schmidt who was my academic host during my fruitful half-a-year research stay at the University of Manchester. Our collaboration inspired and taught me a lot, not to mention that it resulted in a joint paper and a conference talk. I hope that we resume our cooperation soon. In general, I am very grateful to my Manchester colleagues and officemates. Weekly meetings with Renate, Dmitry Tishkovsky and Mohammad Khodadadi entirely devoted to issues put forward by me not only brought a first draft of Chapter 8 (as our joint work) into being, but even more importantly, completely changed my viewpoint on doing science. Once again, many thanks. My Manchester officemates, Fabio Papacchini and Patrick Koopmann in- 
troduced the world of description logics to me. Since I think it is an extremely interesting world, I owe you guys. A single conversation with Ian Pratt-Hartmann appeared to be a real breakthrough in my thinking of modal logics with global counting operators. The inspiration for Theorems 5.16 and 5.17 comes from lan.

Of course, I am also grateful to my Polish colleagues, especially Marek Nowak, for their constant readiness to dispel my doubts. Obviously, much less would have been done if it were not for the financial support from the National Science Centre of Poland. Thanks to the grant I was awarded, I had the opportunity to spend a very fruitful period in Manchester, to attend several important international conferences, and finally, to publish this book.

Finally, a priceless contribution to the book was made by my friend Wojciech Szymański, who I could count on 24 hours a day. Notwithstanding the circumstances, he was always ready to proofread subsequent parts of my work and to introduce countless corrections. I really do not know how I can ever repay him. I will consider lifetime free logic classes.

In the end I want to thank my parents, whose support, encouragement and help were absolutely invaluable in coping with the very down-toearth problems that appeared during my writing. 
Hybrid logics are powerful extensions of modal logics, which, roughly speaking, allow for referring to the semantics of the logic by means of syntactic tools. Augmenting standard modal logics with expressions of an additional sort - nominals, and the so-called satisfaction operators caused a spectacular boost to their expressive power. The satisfaction relation, accessibility statements and equality assertions, hitherto only expressible in meta-language, became available within the object language of the logic. A natural question arose: how do these highly expressive logics behave in terms of decidability? How much must we sacrifice to the altar of this increased expressive power? However, whereas the sole origins of hybrid logics can be traced back to the 1960s and the works of Arthur Prior, some additional "computational" properties of these logics have become a subject of systematic research relatively recently. There has been a lot done since then, though. From the mid-1990s to the present times, a systematic theory of the computational complexity of hybrid logics has been developed by many researchers: Patrick Blackburn, Jerry Seligman, Carlos Areces, Maarten Marx, Thomas Schneider - just to mention a few. Up to now almost all the facts from this area of investigation have been revealed. Theorems placing particular hybrid logics in concrete complexity classes have been established for most of them, defined over most of the well-known frame classes. It seems that not much is left to be done.

This book stands at the intersection of two topics: the decidability and computational complexity of hybrid logics, and the deductive systems designed for them. As pointed out above, there are not many blanks to be filled left in the first field. Nonetheless, if we relax our definition of hybrid logics a bit, which captures them as modal logics involving an additional sort of expressions, a wide area for investigation opens. Taking as a starting point the expressive power of binder-free hybrid logics, we can indicate some variants of nominal-free modal logics whose expressivity matches the expressivity of the former, e.g. 
modal logics with the difference operator $D$ or with global counting operators $\mathrm{E}_{>n}, \mathrm{E}_{<n}$. If we take a closer look at logics augmented with counting modalities, it occurs that they can be seen as non-standard hybrid logics since they allow expressing nominals which are true in exactly one world, in the following way:

$$
i:=\left(\mathrm{E}_{=1} p_{i} \wedge p_{i}\right) .
$$

Also, the satisfaction operators can easily by mimicked by means of global counting operators:

$$
\mathfrak{a}_{i} \varphi:=\left(\mathrm{E}_{=1} p_{i} \wedge \mathrm{E}_{=0}\left(p_{i} \wedge \neg \varphi\right)\right) .
$$

This observation, made by Carlos Areces in [6], enables extending research conducted on hybrid logics also to modal logics with global cardinality restrictions. Apparently, even though systems with numerical constraints have been quite thoroughly investigated within the description logic community, numerous complexity problems remained open. Some of them are raised in this book.

In addition, in the case of deductive systems for hybrid logics a lot is happening on the market. Algorithms of various types have been developed over the last 20 years. Jerry Seligman gave foundations to the theory of sequent calculi for hybrid logics; Miroslava Tzakova and Patrick Blackburn provided the first, respectively, prefixed and internalized tableau-based decision procedures tailored for these logics; and Torben Braüner established a natural deduction system. On the other hand, many interesting tableau calculi for logics with numerical restrictions were invented by description logicians. So, where is the space for further investigations? It turns out that, still, many optimization problems await better solutions. We can set many criteria for evaluation of particular deductive systems: conceptual simplicity, range of application, computational complexity, behaviour in practical performance. Optima in several of these have not been reached by any existing decision procedures for binder-free hybrid logics. That leaves some room for new results. In the case of systems for logics with counting operators, another sort of problem emerges. Unfortunately, in description logics which, contrary to ordinary modal logics, allow so-called role hierarchies (i.e. the set of inclusions between accessibility relations), 
counting operators can only be attached to these roles that are simple (for a precise definition of a role being simple see Section 8.6.2 of Chapter 8) if decidability is to be retained. The universal role - the description logic counterpart of the universal accessibility relation - is not simple. Therefore, we cannot reason about global counting operators if we do not abandon the idea that they are assigned to a certain role. Since most algorithms designed for description logics treat the universal role as a proper role with all its features (like transitivity, symmetry, reflexivity), global cardinality restrictions are outside their scope. This is a gap that asks to be filled.

The book splits into two major parts. The first part is devoted to more "theoretical" issues like the decidability, axiomatizability and computational complexity of hybrid logics. Hybrid logics themselves are divided into two categories: logics involving nominals, called here standard hybrid logics, and logics with global counting operators which we refer to as non-standard hybrid logics. As mentioned in the first part of the introduction, our knowledge on both types is not even. Significantly more decidability and complexity results were established in the area of standard hybrid logics. We therefore present both categories in a consecutive manner. In the first place, we critically expose standard hybrid logics and provide the characterization of their syntax, semantics, expressivity, decidability and computational complexity. We also provide a brief overview of Hilbert-style calculi for particular logics. Subsequently, we proceed to non-standard hybrid logics. We keep the order of presentation from sections devoted to standard hybrid logics. Nonstandard hybrid logics are also the subject of two main contributions of the first part of the book, namely:

1. establishing a sound and complete axiomatization for the modal logic $K$ with global counting operators $\left(\mathcal{M}_{K}\left(E_{n}\right)\right.$ ), which can be easily extended onto other frame classes (Section 5.4),

2. establishing tight complexity bounds, namely NEXPTIME-completeness for the modal logic with global counting operators defined over the classes of arbitrary, reflexive, serial, symmetric and transitive frames $\left(\mathcal{M}_{\mathrm{K}}\left(\mathrm{E}_{n}\right), \mathcal{M}_{\mathrm{T}}\left(\mathrm{E}_{n}\right), \mathcal{M}_{\mathrm{D}}\left(\mathrm{E}_{n}\right), \mathcal{M}_{\mathrm{B}}\left(\mathrm{E}_{n}\right), \mathcal{M}_{\mathrm{K} 4}\left(\mathrm{E}_{n}\right)\right)$, where binary coding is assumed, and establishing the exponential-size model property 
for this logic defined over the classes of Euclidean and equivalential frames $\left(\mathcal{M}_{\mathrm{K} 5}\left(\mathrm{E}_{n}\right), \mathcal{M}_{\mathrm{S} 5}\left(\mathrm{E}_{n}\right)\right)$ (Section 5.5).

The second part of the book consists of a detailed study of particular deductive systems for, subsequently, standard and non-standard hybrid logics. In fact, the alternative title of the present book could be "Tableau-based decision procedures and the decidability problem for hybrid logics" since the vast majority of our considerations are devoted to tableau calculi (to be more precise, save Hilbert-style calculi from the first part and one sequent calculus, all deductive systems discussed in the book are tableau-based). The reason is that tableau calculi, as mostly semantic-based tools, seem to successfully blend modeltheoretic features, which can be interesting from an abstract point of view, and the potential of being implemented, which allows investigating them in many pragmatic respects. The remaining types of deductive systems, like sequent calculi, natural deduction systems or resolutionbased systems do not seem to be so attractive in both fields. In the book we only investigate those tableau-calculi that give foundations for decision procedures. It means that they not only must be sound and complete for a certain logic, but they also need to terminate. That is why blocking mechanisms that ensure termination for the calculi under consideration are one of the predominant topics of the second part of the book. The starting point for devising our tableau-based algorithm for standard and non-standard hybrid logics is the automated synthesis framework presented by Renate Schmidt and Dmitry Tishkovsky in [84]. Their automated procedure of generating tableau calculi is successfully employed in Chapters 7 and 8; nonetheless, for each introduced calculus independent proofs of completeness are given. The most ingenious idea of [84] which we exploit throughout the whole second part of the book is the unrestricted blocking mechanism that, consisting of one elementary rule equivalent to the law of excluded middle and two simple side-conditions, behaves very well when incorporated into tableau calculi for various kinds of logics. In fact, the unrestricted blocking mechanism is a sort of benchmark in our considerations. For all presented systems and blocking techniques employed, we take conceptual simplicity, liability to possible extensions of underlying logics and the 
size of returned models for satisfiable formulas as primary criteria of evaluation.

The main contributions of the second part of the book can be summarized in the following three points:

1. Devising prefixed and an internalized tableau calculi which are sound, complete, and terminating for a rich class of binder-free standard hybrid logics. The established tableau calculi meet the criteria of evaluation presented above. Moreover, for the first time in the literature of the subject the negative rule for the D operator is non-branching (Sections 7.2, 7.3, 7.4 and 7.6),

2. Devising prefixed and an internalized tableau calculi which are sound, complete, terminating for non-standard hybrid logics. The established tableau calculi meet the criteria of evaluation presented above. The internalization technique is novel in the literature (Sections 8.1, 8.2, 8.3 and 8.4),

3. Devising the first hybrid algorithm involving an inequality solver for modal logics with global counting operators (Section 8.6.3).

The book is structured as follows. Chapter 1 introduces the topic of the book and provides a general overview of the following chapters. The first major part of the book, titled "Logical Preliminaries", consists of five chapters. Chapter 2: "Modal logic, decidability and complexity" delivers a brief survey on modal logics and explains basic notions referring to decidability and computational complexity. Chapter 3: "Deductive Systems" introduces the notions of tableau and sequent calculi and explains how they are exploited throughout the book. Chapter 4: "Hybrid Logic" briefly describes hybrid logics, their syntax, semantics, expressive power and computational complexity. Chapter 5: "Logic $\mathcal{M}\left(\mathrm{E}_{n}\right)$ " explains the notion of non-standard hybrid logics, provides their axiomatization and establishes the results on their computational complexity. In Chapter 6, "Remarks on description logics contributions", the terminology and basic facts from the area of description logics are presented.

The second major part, "Deductive systems", splits into two chapters. Chapter 7, "Deductive systems for standard hybrid logics", focuses on deductive systems for hybrid logics we call standard. Two sound, complete and terminating tableau-based decision procedures are provided 
and compared with other existing tableau calculi in terms of blocking mechanisms involved. Chapter 8, "Deductive systems for non-standard hybrid logics", is devoted to tableau-based decision procedures for nonstandard hybrid logics. Again, two sound, complete and terminating calculi are shown. An extensive overview of concurrent decision procedures for logics with cardinality constraints is provided. Some parallels between these systems and the ones introduced in the preceding part of the chapter are drawn. The book is briefly concluded in the Summary. 


\section{Part I}

\section{LOGICAL PRELIMINARIES - HYBRID LOGICS, DECIDABILITY, DEDUCTIVE SYSTEMS}

This part introduces and explains some preliminary notions used throughout the book. The reader is assumed to have some basic knowledge of modal logics, however to a large extent this part of the book may be considered as selfcontained. The main issues discussed are: expressivity, decidability and computational complexity of standard and non-standard hybrid logics. 



\section{2}

MODAL LOGIC, DECIDABILITY AND COMPLEXITY

\subsection{MODAL LOGIC}

For starters, let us quote three slogans with which Patrick Blackburn, Maarten de Rijke and Yde Venema - the authors of one of the most influential textbooks of modal logic - characterize contemporary modal logic (see $[15,11])$ :

SLOGAN 1: Modal languages are simple yet expressive languages for talking about relational structures.

SLOGAN 2: Modal languages provide an internal, local perspective on relational structures.

SLOGAN 3: Modal languages are not isolated formal systems.

Yet, since our aim is not to give a versatile overview of modal logics - in fact, the discussion on standard modal logics only serves further considerations on hybrid logics - let us concisely comment on all three slogans.

First, since the ground-breaking paper of Kripke ([63]) appeared in which he exposes his possible world semantics for modal logics, it has been quite clear that models for these logics can be seen as relational structures (or directed graphs). More precisely, a Kripke model for a modal logic $\mathcal{M}$ is a triple $\langle W, R, V\rangle$, where $W$ is a non-empty set called a universe, $R$ is a binary (or $n$-ary) relation over $W$ called an accessibility relation, and $V$ is a valuation function mapping propositional formulas to subsets of $W$. Elements of $W$ are called worlds, states, points or nodes (in places where it does not lead to a confusion, we use all these names interchangeably). Primarily, elements of $W$ were considered to be possible worlds which are accessible to each other through $R$. However, the contemporary modal logic departs from such strict and narrow alethic understanding of relational structures. 
In fact, we can interpret $W$ and $R$ arbitrarily: as states of a computer linked by the execution of an appropriate program (the case of the multi-modal logic PDL), as states of knowledge of an agent (the case of epistemic logics), or as obligated and permitted states of affairs (the case of deontic logics) ${ }^{1}$. In either case, modal language is a simple and elegant tool for analysing underlying relational structures.

Second, the whole standard modal logic is wrapped around the satisfaction relation $\Vdash$, whose character is intrinsically local. Indeed, the expression:

$$
\mathfrak{M}, w \Vdash \varphi
$$

denotes the fact that $\varphi$ is satisfied at a world $w$ (i.e. locally). For the full inductive definition of the $\Vdash$ relation the reader is referred to Section 4.2. Standard modal languages enable us to penetrate a model from inside, moving along accessibility edges by using modal operators (usually denoted by $\diamond$ and $\square$ ). However, the standard modal logic lacks "a bird's eye view" which would allow to reason about a model as a whole. For instance, first-order sentences that are satisfied by a model refer to the entire model and do not depend on a particular assignment of a value to a variable. In modal logics, whether a formula is satisfied depends on a particular world at which it is evaluated.

Third, thanks to van Benthem ([94]), we know that for many popular frame classes, modal languages defined over these classes can be considered as fragments of $\mathcal{L}^{2}$ (first-order language with two variables) ${ }^{2}$. By means by the so-called standard translation ST we can translate modal formulas into $\mathcal{L}^{2}$ formulas in the following way:

$$
\begin{array}{ll}
\mathrm{ST}_{x}(p) & =P(x), \quad p \in \mathrm{PROP}, \\
\mathrm{ST}_{x}(\neg \varphi) & =\neg \mathrm{ST}_{x}(\varphi), \\
\mathrm{ST}_{x}(\varphi \wedge \psi) & =\mathrm{ST}_{x}(\varphi) \wedge \mathrm{ST}_{x}(\psi), \\
\mathrm{ST}_{x}(\diamond \varphi) & =\exists y\left(\operatorname{Rx} y \wedge \mathrm{ST}_{y}(\varphi)\right) .
\end{array}
$$

1 For a thorough discussion on a wide plethora of possible interpretations of relational structures, see [96]

2 However, if we want to "embed" some frame properties, like transitivity or Euclideaness, in first-order language, $\mathcal{L}^{2}$ does not provide enough expressive power - we need to turn to the language with three or even more variables. As an example of the latter, consider the formula $\square \diamond \diamond \varphi \rightarrow \diamond \varphi$. 
Keeping in mind the restriction from the footnote 2 , we obtain a theorem almost straightforwardly following the definition of ST. Let $[x \longleftarrow w]$ mean the assignment of the world $w$ to the free variable $x$ occurring in $\operatorname{ST}(\varphi)$. Then:

TheOREM 2.1 ([94], see also [11]). For any modal formula $\varphi$, any Kripke model $\mathfrak{M}$ and any world $w$ in $\mathfrak{M}$ we have $\mathfrak{M}, w \Vdash \varphi$ if, and only if $\mathfrak{M}=S T_{x}(\varphi)[x \longleftarrow w]$.

It follows that modal formulas encapsulate a particular fragment of first-order language, namely, these first-order formulas that are invariant under bisimulations.

Why then should we consider modal logics separately, and not as just an ordinary fragment of first-order logic? First of all, they form a nice toolkit for reasoning about relational structures. Second, they behave very well in terms of computational complexity - PSPACE-completeness for the basic modal logic $K$ looks much better than NEXPTIME-completeness for the whole $\mathcal{F} \mathcal{O}^{2}$ (first order logic defined over $\left.\mathcal{L}^{2}\right)([41,3]$ ).

Nevertheless, modal logic has its limitations, even in the context of reasoning about relational structures. It does not allow for an "external reference" to a model. The whole reasoning is conducted from an internal perspective, from which, however, certain discoveries are impossible to make. For instance, an ordinary modal formula $\varphi$ cannot distinguish between two identical (i.e. satisfying the same formulas) states in a model. Besides, we cannot directly refer to concrete points in a model and state that these points satisfy particular formulas or that they are $R$-linked to some other points. Hybrid logics appear to be at least a partial answer to this lack of expressive power of the ordinary modal logic. We get back to and carry on with this discussion in Chapter 4.

\subsection{DECIDABILITY}

We start this short section with recalling three of the possible decision problems for modal logic (cf. [85]):

SATISFIABILITY PROBLEM $\mathcal{L}(\mathcal{F})$-SAT. Given a modal formula $\varphi$, and a class of frames $\mathcal{F}$ determine whether there exists a model $\mathfrak{M}=$ 
$\langle W, R, V\rangle$ based on a frame from $\mathcal{F}$ and a world $w \in W$ such that $\mathfrak{M}, w \Vdash \varphi$.

Global Satisfiability problem $\mathcal{L}(\mathcal{F})$-GSAT. Given a modal formula $\varphi$, and a class of frames $\mathcal{F}$ determine whether there exists a model $\mathfrak{M}=\langle W, R, V\rangle$ based on a frame from $\mathcal{F}$ such that $\mathfrak{M} \Vdash \varphi$, where $\mathfrak{M} \Vdash \varphi$ denotes $\mathfrak{M}, w \Vdash \varphi$ for all $w \in W$.

MOdEL CHECKING PROBLEM $\mathcal{L}(\mathcal{F})$-MC. Given a modal formula $\varphi$ and a finite model $\mathfrak{M}=\langle W, R, V\rangle$ based on a frame from the class $\mathcal{F}$, determine whether there exists a world $w \in W$ such that $\mathfrak{M}, w \Vdash \varphi$.

The most common way of understanding the notion of decidability of a certain logic is boiling it down to the decidability of the satisfiability problem for this logic. In this book we follow this path. We call a modal logic $\mathfrak{M}$ decidable if its satisfiability problem is also decidable. Moving one step forward, a satisfiability problem for a logic is decidable whenever there exists an effective procedure for determining, for each formula $\varphi$ of the language of $\mathcal{M}$, whether $\varphi$ is satisfiable.

The reader might have already noticed that speaking on the decidability of a logic, we do not refer to the notion of validity whatsoever. This is caused by the fact that the validity problem for a logic ${ }^{3}$ is reducible to the satisfiability problem for this logic as its co-problem (complement). Finding a complement for a certain problem consists in reversing yes and no answers. Indeed, if we want to check if a formula $\varphi$ is valid, we need to verify whether its negation is satisfiable, and search for "no" as an answer. All logics discussed in this book are decidable and the presented deductive systems serve as decision procedures for these logics.

\subsection{COMPLEXITY}

In this section, we briefly comment on some of the computational properties of formal systems discussed in this book. Usually, if not indicated otherwise, by the computational complexity of a logic we mean the computational complexity of its satisfiability problem. Boldly speaking,

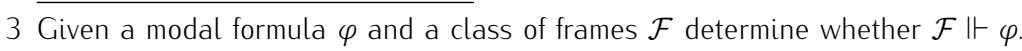


determining the computational complexity of a logic answers the question of how much resources, relatively to the size of a formula $\varphi$, we need to solve the satisfiability problem for $\varphi$. Resources we consider exploitable at performing a solution of the satisfiability problem are time and space. The size of a formula $\varphi$ is usually identified with the number of atomic propositions occurring in $\varphi$. However, if a numerical restriction is involved in a formula $\varphi$, the "propositional" size of $\varphi$ needs to be increased by the value of this restriction (if it is coded in unary) or the logarithm of the value of this restriction (if it is coded in binary).

Most commonly, a precise estimation of the amount (with respect to the size of a formula) of resources we need to solve the satisfiability problem is not possible. We therefore only use some rough complexity classes instead. The complexity classes recalled in this book are: $P, N P$, PSpace, ExpTime and NexpTime. They can be set up in the following hierarchy:

$$
P \subseteq N P \subseteq P S P A C E \subseteq E X P T I M E \subseteq N \text { EXPTIME }
$$

However, the only known strict inclusion between these classes is

$$
P \varsubsetneqq \text { EXPTIME. }
$$

What do all these abbreviations stand for? $P$ is the class of all problems solvable in deterministic polynomial time, NP names the class of all problems solvable in non-deterministic polynomial time, PSPACE denotes the class of all problems that need deterministic polynomial space to get a solution, EXPTIME gathers all problems solvable in deterministic exponential time and, ultimately, NEXPTIME is the class of all problems requiring non-deterministic exponential time to get solved. We still do not know what these mysterious names of classes mean. Let's start with a rough, informal explanation. If a problem belongs to $\mathrm{P}$, an algorithm that solves it is deterministic and terminates after a time bounded by a function which is polynomial in the size of an input (of a formula in the case of algorithms checking the satisfiability). If it belongs to NP, it means that an algorithm solving it is non-deterministic and terminates after a time bounded by a function which is polynomial in the size of an input. The non-determinism of an algorithm, pictorially, implies that whenever an algorithm ought to make a choice, it makes 
the right choice, as if it were using a "magic wand". For instance, if in a tableau derivation a splitting (e.g. $(V)$ ) rule has to be applied, an algorithm chooses a correct branch to carry on the derivation. If a problem is in PSPACE, a solving algorithm requires a space bounded by a function which is polynomial in the size of an input. The classes ExPTIME and NExpTIME can be explained in a similar way to $P$ and NP. Let's get to a more formal way of putting it forward. The reader is assumed to be familiar with the notion of a Turing machine (TM).

The problems of decidability and computational complexity (also with regard to logics) are discussed exhaustively in [74]. However, a concise and very handy introduction to decidability and complexity of modal logics can be found in the Appendix C of [15].

Definition 2.1 ([15]). A deterministic TM is polynomially time bounded if there is a polynomial $p(n)$ such that the machine always halts after at most $p(n)$ steps, where $n$ is the length of the input. a problem is solvable in polynomial time if there is a polynomially bounded $\mathrm{TM}$ that solves it. The class of all problems solvable in polynomial time is called $P$.

Definition 2.2 ([15]). A non-deterministic TM is polynomially time bounded if there is a polynomial $p(n)$ such that no computation of the machine continues for more than $p(n)$ steps where $n$ is the length of the input. NP is the class of all problems decided by a polynomially bounded non-deterministic TM.

Definition 2.3 ([15]). A deterministic TM is polynomially space bounded if there is a polynomial $p(n)$ such that no computation of the machine scans more than $p(n)$ tape squares, where $n$ is the length of the input. PSPACE is the class of all problems that are decided by a polynomially space bounded deterministic TM.

Now, since considerations devoted to the computational complexity involve the notions of membership in a complexity class and hardness, a few words of explication are in place. Boldly, if a problem is a member of a complexity class, it needs at most as many steps as indicated by this class to get solved. If it is C-hard, where $C$ is the name of a complexity class, then it requires at least as many steps as indicated by this class 
to get the solution. C-hardness and membership in $\mathrm{C}$ of a problem together establish $\mathrm{C}$-completeness. Again, more formally:

Definition 2.4 ([15]). Let $P_{1}, P_{2} \subseteq \Sigma^{*}$, where $\Sigma^{*}$ is a set of words, be problems, and $\Sigma$ - an alphabet. A polynomial time computable function $f: \Sigma^{*} \rightarrow \Sigma^{*}$ is called a polynomial time reduction from $P_{1}$ to $P_{2}$ if for each $s \in \Sigma^{*}$ we have that $s \in P_{1}$ if, and only if $f(s) \in P_{2}$.

Definition 2.5 ([15]). Let $C$ be a class of problems. A problem $P$ is $\mathrm{C}$-hard (with respect to polynomial time reductions) if every problem in $C$ is polynomial time reducible to $P$. I.e. there exists a polynomial time reduction from every problem in $C$ to $P$. $P$ is $C$-complete if it is $\mathrm{C}$-hard and moreover $P \in \mathrm{C}$. That is, the $\mathrm{C}$-complete problems are the hardest problems in $\mathrm{C}$.

Throughout the book we use two techniques to establish a particular complexity class for a problem: encoding of bounded tiling problem in a logic to establish its NExPTIME-hardness and proving that a logic enjoys the exponential-size model property to show its membership in NEXPTIME. Whereas the former is thoroughly explained in Section 5.17, the latter deserves a comment here. Assume that a logic $\mathcal{M}$ has the exponential-size model property. Then for each satisfiable formula $\varphi$ of the language of $\mathcal{M}$ we can construct, in a non-deterministic way, a model whose size is exponential in the size of a formula. The nondeterminism of this procedure assures that, indeed, we built a model satisfying $\varphi$. Verifying the fact that our construction is a model for $\varphi$ requires an exponential number of steps. 



\section{DEDUCTIVE SYSTEMS}

Since a major part of the book is devoted to concrete deductive systems for hybrid logics, providing a formal definition of a deductive system is in place. We use this notion in a similar way to Indrzejczak in [53], therefore, we repeat his very general definition of a deductive system.

Definition 3.1 (Deductive system ([53])). Every deductive system may be characterized on two elementary levels of description:

- the calculus,

- the realization.

By a calculus we understand a non-empty set of schemata of rules of the form:

$$
X_{1}, \ldots, X_{n} / Y_{1}, \ldots, Y_{m}, \quad n \geq 0, m \geq 1,
$$

with a possible list of side conditions. Symbols $X_{i}$, denote some data structures being transformed into data structures $Y_{i}$.

By a realization we usually understand the set of instructions of how to apply rules to perform a derivation/construct a proof.

In the book, we describe instantiations of two basic types of deductive systems: tableau calculi and sequent calculi. We dedicate to each of these types a short, introductory note. We skip all historical remarks as hardly relevant for our considerations. We also waive the idea of a profound exposition of Hilbert-style calculi, even though several such calculi are presented in this book. We assume that the notion of Hilbert-style calculus is rather familiar to most readers and we consider its thorough examination as lying outside the scope of the book. Considerations in this section are based on $[53,52]$. 


\subsection{SEQUENT CALCULI}

Originally invented by Gentzen in the 1930s, sequent calculi are finite sets of primitive rules of the form:

$$
S_{1}, \ldots, S_{n} / S_{n+1}
$$

where $S_{i}$ is a sequent schema.

By a sequent we understand an ordered pair of finite sequences of formulas with a symbol $\Rightarrow$ between these sequences. Every sequent is therefore of the form:

$$
\varphi_{1}, \ldots, \varphi_{n} \Rightarrow \psi_{1}, \ldots, \psi_{m} \quad n, m \geq 0
$$

A sequence standing in a sequence on the left hand-side of $\Rightarrow$ is called an antecedent of a sequent whereas a sequence which stands on the right-hand side of $\Rightarrow$ is called a succedent of a sequent. We can, but do not have to, interpret $\Rightarrow$ as a deducibility relation $\vdash$. According to the standard understanding of a sequent, formulas on the left-hand side of $\Rightarrow$ can be seen as a conjunction, whereas the ones standing on the right hand-side as a disjunction.

A sequent calculus consists of the schema of the so-called axiomatic sequent of the form:

$$
\varphi \Rightarrow \varphi
$$

and a set of rules that allow to derive a sequent out of a finite set of sequents. These rules split into structural rules and logical rules. All rules, save cut, go in pairs in case we want to introduce a formula to an antecedent or to a succedent of a sequent. Structural rules are: the (cut) rule, the $\mathrm{W}$ (weakening) rules, the $\mathrm{C}$ (contraction) rules and the $(P)$ (permutation) rules. E.g. the rule $(\Rightarrow W)$ (weakening of the succedent) has the form:

$$
\frac{\Gamma \Rightarrow \Delta}{\Gamma \Rightarrow \Delta, \varphi}
$$

and indicates (under the assumption that $\Rightarrow$ is understood as $\vdash$ ) that if we can derive a set $\Delta$ of formulas from a set $\Gamma$, we can also derive every superset of $\Delta$. This also makes sense if we keep in mind that $\Delta$ is in this case interpreted as $\bigvee \Delta$. 
Logical rules help us to handle logical operators (connectives, modal operators), i.e. to introduce them to an antecedent or a succedent of a sequent. For example, the pair of rules $(\wedge \Rightarrow)$ and $(\Rightarrow \wedge)$ for conjunction looks in the following way:

$$
\frac{\varphi, \psi, \Gamma \Rightarrow \Delta}{\varphi \wedge \psi, \Gamma \Rightarrow \Delta} \quad \frac{\Gamma \Rightarrow \Delta, \varphi \quad \Gamma \Rightarrow \Delta, \psi}{\Gamma \Rightarrow \Delta, \varphi \wedge \psi}
$$

In the rules above, the sets $\Gamma$ and $\Delta$ form a context.

A whole derivation process consists in a proof search for a formula $\varphi$. We always start with instances of the axiomatic sequent scheme. $\varphi$ is valid if a sequent-based procedure at some point returns a sequent with a sole $\varphi$ in a succedent and with an empty antecedent.

\subsection{TABLEAU CALCULI}

Let's imagine that we turn a sequent derivation upside-down, i.e. we start our derivation from a formula under question and finish with the atomic formulas or a clash symbol $(\perp)$. Roughly speaking, tableau derivations are performed in this way.

This, however, is jumping ahead. Let's begin with introducing the notion of a tableau calculus as a set of derivation rules of the form:

$$
\frac{X_{1}, \ldots, X_{n}}{Y_{1}|\cdots| Y_{m}}, \quad n \geq 0, m \geq 1,
$$

where $X_{i}$ denote some data structures being transformed into data structures $Y_{i}$. In most most tableau calculi $X_{i}$ and $Y_{i}$ stand for single formulas. The $\mid$ symbol in the denominator of a rule stands for splitting of a derivation. We can divide our tableau rules with respect to various criteria. First, we can distinguish decomposition rules, clash rules and, let's call them, auxiliary rules. Decomposition rules help us to deal with complex formulas and decompose them into simpler ones. Clash rules provide the conditions for obtaining a clash (an inconsistency) in a branch of a tableau. Auxiliary rules might help to deal with, e.g., some meta-language symbols used in a calculus (like the equality symbol) or with frame conditions for an underlying logic.

Following Smullyan ([89]) and Kaminski ([55]), we can also divide rules with respect to the number of occurrences of $\mid$ symbol in a denominator of a rule. Rules with no occurrence of $\mid$ symbol in a denominator 
are called $\alpha$ rules or deterministic rules. If $\mid$ occurs in a denominator at least once, the rule is labelled as $\beta$ rule, or called non-deterministic (other: splitting, branching).

Among tableau calculi themselves we can distinguish between analytical tableau calculi (also called declarative) and constructive tableau calculi (also called procedural). The former approach defines a tableau as a derivation tree in which nodes represent formulas or sets of formulas to which certain tableau rules were applied. When reasoning about modal logics, we realize that except formulas under consideration, we introduce another parameter to a derivation, namely a world in which a formula is evaluated. This is done by assigning to formulas labels that represent worlds of evaluation. These labels can be externalized, meta-language expressions (like in Gabbay's approach in [35]), or internalized whenever an object language of a logic is rich enough to express its own semantics (like in Blackburn's approach in [10]). Analytical tableau calculi are usually cumulative, which means that whenever an expression occurs in a branch, it is never deleted. Whenever a derivation results in an open tableau, a model can be extracted and built out of expressions occurring in an open branch.

In the constructive approach, a tableau is defined differently, namely, it is considered as an abstraction of a model. The existence of the former is proven equivalent to the existence of the latter. During a derivation performed by a constructive tableau algorithm, a completion graph is built which can be considered as an approximation of a tableau. The constructive approach uses external labelling. Labels for worlds are simply represented by names of nodes in a graph. Constructive tableau calculi are often non-cumulative, as they allow removing from a graph previously introduced nodes.

An analytical tableau calculus can always be turned into a constructive one by a suitable refinement. Concrete, detailed examples of both types of calculi are thoroughly presented in Chapters 7 and 8.

Since in this book we only cope with decidable logics, we want to turn tableau calculi we operate on into decision procedures. The crucial point in this case is the termination of such a calculus. If a calculus terminates, it means that for every input formula $\varphi$ it is determined in a finite number of steps whether a formula is satisfiable. For some logics no additional mechanisms beyond the ordinary tableau rules are 
necessary for ensuring termination of a calculus suited for them. There exist, however, logics whose expressive power interferes with the termination of a calculus designed for them. Calculi for such logics require augmentation with an extra mechanism called blocking. Finding an appropriate blocking mechanism for a calculus, i.e. the one that secures the termination and simultaneously does not break the completeness of a calculus, is often the most difficult task. In fact, most frequently different tableau calculi for the same logic are distinguished by their blocking mechanisms rather than by the rules whose shape is often a straightforward consequence of the semantics of such a logic.

Let's now consider the definitions of soundness and completeness of a tableau calculus which will occur in Chapters 7 and 8. In general, a tableau calculus is sound if for each satisfiable input formula each tableau is open, i.e., there exists a fully expanded branch in which no clash rule was applied. A tableau calculus is called complete if for each unsatisfiable input formula there exists a closed tableau, i.e. a tableau where a clash rule was applied in each branch. Both definitions correspond to the standard definitions of soundness and completeness known from Hilbert-style calculi. Taking the contraposition of the former, a (Hilbert-style) calculus is sound if, and only if, whenever a formula has a model, it is also consistent (with respect to this calculus). Using the terminology from tableaux, whenever a formula is satisfiable, it generates no clashes in some branch of the derivation. The case of completeness is similar. If a formula is unsatisfiable (i.e. its negation is a tautology), it is also inconsistent with respect to a tableau calculus, i.e. it generates clashes in all branches of the derivation.

We now can get back to our point from the beginning of this section. A tableau derivation is the process of searching a model for an input formula. It is, therefore, a decision procedure for satisfiability rather than validity problem. If we want to decide whether a formula $\varphi$ is valid, we turn our tableau-based procedure into a refutation one. We simply need to put $\neg \varphi$ at the initial node of a tableau and verify whether a tableau derivation refutes all attempts of model construction for $\varphi$ by applying a clash rule in each branch.

Further details of both sequent and tableau calculi are discussed in the forthcoming chapters when particular instantiations of these types of deductive systems are presented. 



\section{4}

HYBRID LOGIC

This chapter provides a concise survey on hybrid logics, including their expressive power, axiomatization, decidability and computational complexity. It also sketches a historical overview of the development of these logics.

\subsection{MOTIVATION}

Suppose that a modal logic $\mathcal{M}$ is defined over the signature $\left\langle\mathrm{PROP}, R, R^{-}\right\rangle$, where PROP is a denumerable set of propositional variables $\left\{p_{1}, p_{2}, \ldots\right\}$ and $R^{-}$is the inverse of the relation $R$. In fact, we can consider $\mathcal{M}$ as a temporal logic, so $R$ is a strict total order without endpoints. We can express the thought "in the future it will be cold" by a formula:

\section{$\mathrm{F} \varphi$,}

where $\varphi$ stands for "it is cold". As mentioned in Section 2.1, modal formulas do not distinguish between worlds satisfying formulas under the scope of a modal operator. Consequently, we do not know whether the $F$ sends us three days or three years ahead. By means of the standard modal logic (or standard temporal logic), we cannot express the thought "on John's birthday it will be cold" or "on September 15th it will be cold" since these sentences contain direct pointers that distinguish particular worlds in a model ("John's birthday", "September 15th") and, as such, have some kind of global flavour. If we introduced an additional sort of expressions each of which would label exactly one point in a model, we would be able to express all the thoughts quoted above.

Hybrid logic introduces such expressions and calls them nominals. Each of them indeed holds at exactly one world in a model (although many of them can hold at the same world). Additionally, it augments the basic modal language with the so-called satisfaction operators @ $@_{i}$ that enable us to retrieve all information from a world labelled by $i$. Note 
that by that means we can express the equality between worlds. For example, assume that $i$ means "the day of birth of my child" and $j$ stands for "September 15th". Then the formula @ $i j$ expresses the fact that the day of birth of my child is September 15th. In an analogous way we can express the time relation (or any relation that interprets the symbol $R$ from our signature) between points. For instance, by stating @ $\mathfrak{i}_{i}$ we indicate that my child was born (will be born) before September 15th.

With the aim of further increasing the expressive power of a hybrid language, we can introduce state variables and binders $\downarrow$ and $\exists$ that allow, respectively, for binding a state variable to the point of current evaluation of a formula, or quantify over all points in a model. A more precise formulation of the semantics of these operators can be found in the next section.

To sum up, the motivation standing behind hybrid logic is to cross the expressivity chasm between modal and first-order logic, whilst at the same time retaining nice properties of the former like the conceptual simplicity or a low computational complexity. We should see in Section 4.5 that although introducing nominals and the @ operators hardly involve some additional complexity costs, the case is different with the binders which make the logic undecidable.

For further comments and a more profound discussion on the informal motivation for hybrid logics see $[21,9,11]$.

\subsection{SYNTAX AND SEMANTICS}

Before we proceed to a less formal description of the hybrid language, we provide definitions of the syntax and the semantics of the full hybrid language.

Definition 4.1 (Syntax of the full hybrid language). Let PROP = $\left\{p_{1}, p_{2}, \ldots\right\}$ be a countable set of propositional letters, NOM = $\left\{i_{1}, i_{2}, \ldots\right\}$ be a countable set of nominals and $\operatorname{SVAR}=\left\{x_{1}, x_{2}, \ldots\right\}$ be a countable set of state variables. We define a set FORM of formulas of full hybrid language as follows:

$$
\text { FORM }:=p|i| x|\neg \varphi| \varphi \wedge \psi|\nabla \varphi| @_{i}\left|@_{x} \varphi\right| \mathrm{E} \varphi|\mathrm{D} \varphi| \downarrow x . \varphi \mid \exists x \varphi,
$$

where $p \in \operatorname{PROP}, i \in \mathrm{NOM}, x \in \operatorname{SVAR}$ and $\varphi, \psi \in$ FORM. 
We give explicit definitions of other Boolean connectives and modal operators ${ }^{1}$ :

$$
\begin{array}{ll}
\varphi \vee \psi:=\neg(\neg \varphi \wedge \neg \psi), & \mathrm{A} \varphi:=\neg \mathrm{E} \neg \varphi, \\
\varphi \rightarrow \psi:=\neg(\varphi \wedge \neg \psi), & \overline{\mathrm{D}} \varphi:=\neg \mathrm{D} \neg \varphi, \\
\square \varphi:=\neg \vee \neg \varphi, & \forall x \varphi:=\neg \exists x \neg \varphi .
\end{array}
$$

(a) and $\downarrow$ are self-dual.

Definition 4.2 (Semantics). A model $\mathfrak{M}$ for the full hybrid logic is a triple $\langle W, R, V\rangle$ where:

$W \neq \varnothing$ is called a domain,

$R \subseteq W^{2}$ is called an accessibility relation,

$V:$ PROP $\cup$ NOM $\longrightarrow \mathcal{P}(W)$ such that for each $i \in \operatorname{NOM} V(i)$ is a singleton set; $V$ is called a valuation function.

Given a model $\mathfrak{M}=\langle W, R, V\rangle$ and an assignment $g:$ sVAR $\longrightarrow W$, the relation $\Vdash$ is defined inductively:

$$
\begin{array}{ll}
\mathfrak{M}, g, w \Vdash p & \Leftrightarrow w \in V(p), \quad p \in \text { PROP, } \\
\mathfrak{M}, g, w \Vdash i & \Leftrightarrow\{w\}=V(i), \quad i \in \text { NOM, } \\
\mathfrak{M}, g, w \Vdash x & \Leftrightarrow w=g(x), \quad x \in \text { SVAR, } \\
\mathfrak{M}, g, w \Vdash \neg \varphi & \Leftrightarrow \mathfrak{M}, g, w \| \varphi,
\end{array}
$$$$
\mathfrak{M}, g, w \Vdash \varphi \wedge \psi \Leftrightarrow \mathfrak{M}, g, w \Vdash \varphi \text { and } \mathfrak{M}, g, w \Vdash \psi \text {, }
$$

$\mathfrak{M}, g, w \Vdash \nabla \varphi \Leftrightarrow$ there exists $z$ in $W$ such that $w R z$ and $\mathfrak{M}, g, z \Vdash \varphi$,

$\mathfrak{M}, g, w \Vdash @_{i} \varphi \Leftrightarrow$ there exists $z$ in $W$ such that $\{z\}=V(i)$ and $\mathfrak{M}, g, z \Vdash \varphi$,

$\mathfrak{M}, g, w \Vdash @_{x} \varphi \Leftrightarrow$ there exists $z$ in $W$ such that $z=g(x)$ and $\mathfrak{M}, g, z \Vdash \varphi$,

$\mathfrak{M}, g, w \Vdash E \varphi \quad \Leftrightarrow \quad$ there exists $z \in W$ such that $\mathfrak{M}, g, z \Vdash \varphi$,

$\mathfrak{M}, g, w \Vdash D \varphi \quad \Leftrightarrow \quad$ there exists $z \in W$ such that $\mathfrak{M}, g, z \Vdash \varphi$ and $z \neq w$,

$\mathfrak{M}, g, w \Vdash \downarrow x . \varphi \quad \Leftrightarrow \quad \mathfrak{M}, g^{\prime}, w \Vdash \varphi$, where $g \stackrel{x}{\sim} g^{\prime}$ and $w=g^{\prime}(x)$,

$\mathfrak{M}, g, w \Vdash \exists x \varphi \quad \Leftrightarrow \quad$ there exists $g^{\prime}$ such that $g \stackrel{x}{\sim} g^{\prime}$ and $\mathfrak{M}, g^{\prime}, w \Vdash \varphi$,

1 They can serve as rewrite rules in the tableau calculi. 
where $g^{\prime}$ such that $g \stackrel{x}{\sim} g^{\prime}$ is called an x-variant of $g$ and differs from $g$ at most on a value it assigns to $x$.

Comparing the ordinary modal language and the full hybrid language, we realize that two additional sorts of expressions are introduced to the latter: nominals and state variables. It is easy to note that they behave like, respectively, first-order constants and first-order variables. As such, they can be considered as labels for worlds - they enable a direct reference to states of a model within the language of the logic. It, therefore, abandons the local point of view - so characteristic for modal logic (see Section 2.1) - for the first-order, global point of view from which it can scan a semantic structure for itself. Now is a good point to answer the question: in what sense is hybrid logic hybrid? First, it is hybrid since it incorporates the traditional firstorder apparatus of variables and operators that bind variables into the classical modal landscape and merges the standard modal semantics with the Tarskian first-order semantics. Second, and probably more importantly, it is hybrid because its syntax considers both sorts of expressions - propositions and names (i.e. nominals and state variables) - to be well formed formulas. We can say that in this second respect hybrid logic is hybrid because nominals and state variables combine two roles: they are both formulas and terms (names labelling worlds) (cf. $[12,11,13]$ ).

To shed some light on the semantics of the full hybrid language, we present a standard translation of hybrid formulas into $\mathcal{L}^{2}([9])$

$$
\begin{aligned}
& \mathrm{ST}_{x}(i)=x=x_{i}, \quad i \in \mathrm{NOM}, \\
& \mathrm{ST}_{x}(y)=x=y, \quad y \in \mathrm{SVAR}_{\text {, }} \\
& \mathrm{ST}_{x}\left(@_{i} \varphi\right)=\left(\mathrm{ST}_{x}(\varphi)\right)\left[x_{i} / x\right] \text {. } \\
& \mathrm{ST}_{x}\left(@_{y} \varphi\right)=\left(\mathrm{ST}_{y}(\varphi)\right) \text {, } \\
& \mathrm{ST}_{x}(\downarrow y \cdot \varphi)=\exists y\left(x=y \wedge \mathrm{ST}_{x}(\varphi)\right) \text {, } \\
& \mathrm{ST}_{x}(\exists y \varphi)=\exists y\left(\mathrm{ST}_{x}(\varphi)\right) \text {. }
\end{aligned}
$$

The interpretation of nominals and state variables is rather obvious. The $\downarrow x$ operator binds a variable $x$ to the current world, i.e. it assigns the current worlds as a value of $x . \exists x$ operates like the classical first-order existential quantifier. It states that there exists a world which taken as 
a value assigned to $x$ verifies a formula under the scope of $\exists x$. Whereas $\exists x$ acts like a "storage" operator (it enables keeping particular states of a model in memory - see [13]), @ $@_{i}$ and @ $@_{x}$ are "retrieval" operators and allow scanning information from a world labelled by, respectively, $i$ or $x$. More boldly, @i, @x formulas state that at a world labelled by, respectively, $i$ or $x$ a particular formula holds. Note that @ is not a binder.

\subsection{EXPRESSIVITY}

The expressive power of the full hybrid language significantly extends the expressive power of the ordinary modal language. Before concrete examples of hybrid language expressivity are shown, let's distinguish between two types of formulas. Let formulas with only nominals occurring as atomic symbols be called pure formulas. Let formulas involving (a) operators as the main operator be called satisfaction formulas.

\subsubsection{Expressive power}

\section{First-order frame conditions}

As it is commonly known, not every first-order definable frame class is also modally definable. For instance, irreflexivity or asymmetry are not modally characterizable. However, if we augment our language with nominals, then, exploiting pure formulas, we can express a number of frame properties not expressible when only using standard modal language (see [10]):

$$
\begin{array}{ll}
i \rightarrow \neg \diamond i & \text { irreflexivity, } \\
i \rightarrow \square \neg \diamond i & \text { asymmetry, } \\
i \rightarrow \square(\diamond i \rightarrow i) & \text { antisymmetry, } \\
\diamond \diamond i \rightarrow \neg \diamond i & \text { intransitivity, } \\
\diamond i & \text { universality. }
\end{array}
$$

If we also allow satisfaction formulas, we can additionally get the condition for trichotomous relation:

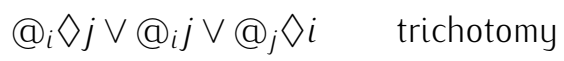




\section{Second-order frame conditions}

If we use the mixed power of temporal logic with two modalities $F$ and $P$ reverse to each other, and hybrid logic, we can even extend firstorder definability and characterize (up to isomorphism) $\mathbb{Z}$. Thanks to van Benthem ([95]), we know that the formula:

$$
(\mathrm{H}(\mathrm{H} p \rightarrow p) \rightarrow(\mathrm{PH} p \rightarrow \mathrm{H} p)) \wedge(\mathrm{G}(\mathrm{G} p \rightarrow p) \rightarrow(\mathrm{FG} p \rightarrow \mathrm{G} p))
$$

defines $\mathbb{Z}$ over strict total orders without endpoints. However, strict total orders are not definable when we confine ourselves to the ordinary modal language. But yet if we augment the language with nominals and the @ operators, we are able to express irreflexivity and trichotomy - the only missing conditions for defining a strict total order (the remaining two: transitivity and lack of endpoints are expressible with ordinary temporal formulas: FF $p \rightarrow F p$ and $F \top \wedge P \top$ ). Thus, we define $\mathbb{Z}$, which is not possible in first-order language.

\section{Equality and accessibility statements}

It is extremely easy to notice that pure formulas and satisfaction formulas enable us to express equality between worlds, or the fact that two worlds are linked by an accessibility edge. The equality between states labelled, respectively, by $i$ and $j$ is expressed by the formula:

\section{$@_{i} j$.}

The fact that worlds labelled, respectively, by $i$ and $j$ are linked by an accessibility edge can be expressed by the formulas:

$$
@_{i} \diamond j \text { or } i \wedge \diamond j \text {. }
$$

\section{Restricting cardinality of universe}

If we want to limit the cardinality of the universe to $n$ states, we can achieve it with the following formula (added to the set of axioms):

$$
@_{i_{1}}\left(\neg i_{2} \wedge \cdots \wedge \neg i_{n+1}\right) \rightarrow \bigvee_{2 \leq k<l \leq n+1} @_{i_{k}} i_{l} .
$$


The same can be done if we define the formula involving the universal modality (it can only be satisfied by models of the cardinality $n$ ):

$$
\mathrm{A}\left(i_{i} \vee \cdots \vee i_{n}\right) \wedge \mathrm{A}\left(\bigwedge_{1 \leq k<l \leq n} @_{i_{k}} \neg i_{l}\right)
$$

Full first-order expressive power

In fact, we can regain the full first-order expressive power out of the full hybrid language. In order to demonstrate it, we define the following translation function named the hybrid translation

$$
\begin{aligned}
& \mathrm{HT}_{x}(x R y)=@_{x} \triangleright y, \\
& \mathrm{HT}_{x}(P(x))=@_{x} p, \\
& \mathrm{HT}_{x}(x=y)=@_{x} y, \\
& \mathrm{HT}_{x}(\neg \varphi) \\
& \mathrm{HT}_{x}(\varphi \wedge \psi)=\neg \mathrm{HT}(\varphi), \\
& \operatorname{HT}_{x}(\exists y \varphi)=\operatorname{HT}(\varphi) \wedge \mathrm{HT}(\psi), \\
& \operatorname{HyH}_{y}(\varphi) .
\end{aligned}
$$

Since we obtained the full first-order expressivity (over the signature $\langle\mathrm{PROP}, R\rangle)$, we can expect that it interferes with the decidability of the full hybrid logic. That is indeed the case which we show in Section 4.5.

\subsubsection{Hierarchy of expressive power}

Figure 1 presents the hierarchy of hybrid languages with respect to their expressive power ([85]). It is clear that some operators are interdefinable, hence not all possible combinations of operators are displayed.

All red nodes represent languages of undecidable hybrid logics. The straightforward conclusion is that the involvement of any hybrid binder results in an undecidable logic. As it turns out in Section 4.5, it is in fact (almost) the case. The undecidability result for hybrid logics with binders is the reason why we confine ourselves to the binder-free hybrid logics in this book. It means that the operators we consider in 


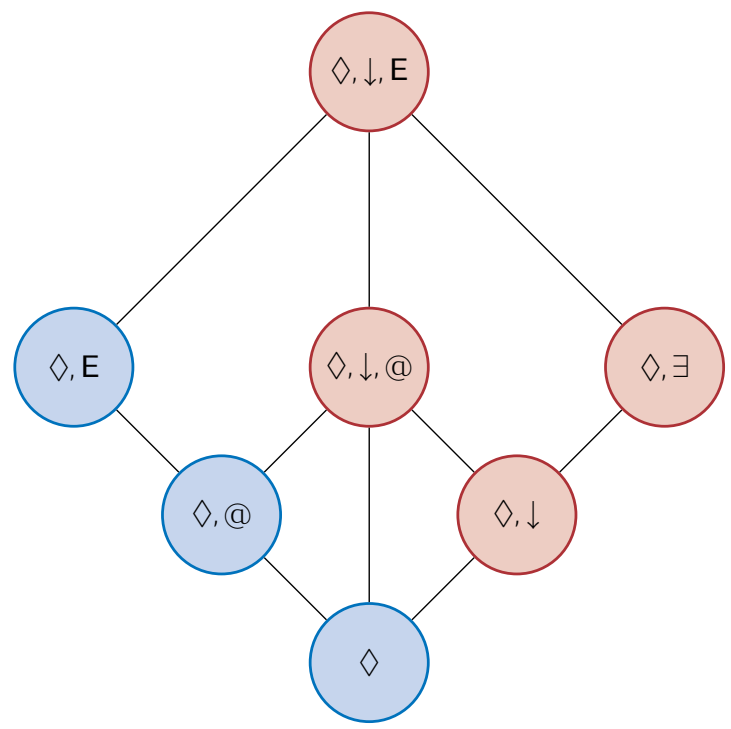

Figure 1: Hierarchy of hybrid languages with respect to their expressive power

connection with hybrid logics are: E, D and @. Of course, they are also mutually interderivable in the way shown below:

$$
\begin{array}{ll}
@_{i} \varphi & =\mathrm{E}(i \wedge \varphi), \\
\mathrm{E} \varphi & =\varphi \vee \mathrm{D} \varphi, \\
i & =p_{i} \wedge \overline{\mathrm{D}} \neg p_{i},
\end{array}
$$

where $p_{i}$ is a fresh propositional variable and $\bar{D}$ denotes the operator dual to D (its intuitive meaning is: "at all worlds different than the current one").

One may have noticed that $\mathrm{D}$ has the greatest expressive power since it can define both other operators and nominals.

\subsection{HILBERT-STYLE CALCULI}

In this section, sound and complete axiomatizations for the logics $\mathcal{H}_{K}$, $\mathcal{H}_{K}$ (@) and $\mathcal{H}_{K}(E)$ are presented ${ }^{2}$. Together with the inference rules

2 We did not manage to find the axiomatization for the logic $\mathcal{H}_{K}(D)$ in the literature of the subject. However, taking into account the axiomatizations for the logics $\mathcal{H}_{K}, \mathcal{H}_{K}(@)$ 
they form standard Hilbert-style calculi for these logics. Let's start with some auxiliary definitions:

Definition 4.3 (General frame $([24]))$. A general frame is a pair $\langle\mathfrak{F}, \mathbb{A}\rangle$, where $\mathfrak{F}=\langle W, R\rangle$ is a frame and $\mathbb{A} \subseteq \mathcal{P}(W)$ such that $W \in \mathbb{A}$ and $\mathbb{A}$ is closed under complement, finite intersection and $R$-successorship. Moreover, the general frame $\langle\mathfrak{F}, \mathbb{A}\rangle$ is:

Differentiated if for all $w, v \in W$ such that $w \neq v$ there is an $A \in \mathbb{A}$ such that $w \in A$ and $v \notin A$,

TIGHT if for all $W, v \in W$ such that $\langle w, v\rangle \notin R$ there is an $A \in \mathbb{A}$ such that $v \in A$ and $w \notin R(A)$,

COMPACT if every $\mathbb{A}^{\prime} \subseteq \mathbb{A}$ with the finite intersection property has a non-empty intersection

DESCRIPTIVE if it is differentiated, tight and compact.

DEFINITION 4.4 (Two-sorted general frame ([24])). A two-sorted general frame is a structure $\mathfrak{F}=\langle W, R, \mathbb{A}, \mathbb{B}\rangle$, where $\langle W, R, \mathbb{A}\rangle$ is a general frame, $\mathbb{B} \subseteq W$ is non-empty and for all $w \in \mathbb{B},\{w\} \in \mathbb{A}$.

and $\mathcal{H}_{K}(\mathrm{E})$, and axioms inherently following from the semantics for $\mathrm{D}$ (see [28]), we can conjecture that the following axiomatization will be sound and complete for the logic $\mathcal{H}_{K}(\mathrm{D})$ with respect to two-sorted descriptive frames

CT All classical tautologies,

$\mathrm{K} \quad \square(\varphi \rightarrow \psi) \rightarrow(\square \varphi \rightarrow \square \psi)$,

$\mathrm{K}_{\mathrm{D}} \quad \overline{\mathrm{D}}(\varphi \rightarrow \psi) \rightarrow(\overline{\mathrm{D}} \varphi \rightarrow \overline{\mathrm{D}} \psi)$,

Sym $_{\mathrm{D}} \quad \overline{\mathrm{D}} \varphi \rightarrow \varphi$,

PsTrans $_{D} \quad \operatorname{DD} \varphi \rightarrow(\varphi \vee \mathrm{D} \varphi)$,

Incl $\diamond \quad \nabla \varphi \rightarrow(\varphi \vee \mathrm{D} \varphi)$,

Incl $_{i} \quad i \vee \mathrm{D} i$,

$\mathrm{Excl}_{i} \quad i \rightarrow \overline{\mathrm{D}} \neg i$,

Nom $_{\mathrm{D}} \quad \mathrm{D}(i \wedge \varphi) \rightarrow \overline{\mathrm{D}}(i \rightarrow \varphi)$.

The rules of inference of $\mathcal{H}_{K}$ are:

MP If $\vdash \varphi \rightarrow \psi$ and $\vdash \varphi$ then $\vdash \psi$,

$\mathrm{N}_{\mathrm{D}} \quad$ If $\vdash \varphi$ then $\vdash \overline{\mathrm{D}} \varphi$ for $i \in$ NOM. 
Definition 4.5 (Two-sorted general descriptive frame ([24])). A twosorted general frame $\langle W, R, \mathbb{A}, \mathbb{B}\rangle$ is descriptive if $\langle W, R, \mathbb{A}\rangle$ is descriptive in the traditional sense.

Definition 4.6 (Two-sorted general descriptive frame ([24])). A twosorted general frame $\langle W, R, \mathbb{A}, \mathbb{B}\rangle$ is strongly descriptive if it is descriptive and satisfies the following further conditions:

1. for all $X \in \mathbb{A}$, if $X \neq \varnothing$, then $X \cap \mathbb{B} \neq \varnothing$,

2. for all $X \in \mathbb{A}$ and $w \in \mathbb{B}$, if $\{v \in X \mid w R v\} \neq \varnothing$, then $\{v \in X \mid$ $w R v\} \cap \mathbb{B} \neq \varnothing$.

Definition $4.7\left(\mathcal{H}_{K}\right.$ axiomatization $\left.([24])\right)$. The axioms of $\mathcal{H}_{K}$ are all instances of the following schemata

CT All classical tautologies,

$\mathrm{K} \quad \square(\varphi \rightarrow \psi) \rightarrow(\square \varphi \rightarrow \square \psi)$,

Nom $\quad \nabla^{n}(i \wedge \varphi) \rightarrow \square^{m}(i \rightarrow \varphi)$, for $n, m \in \mathbb{N}$

The rules of inference of $\mathcal{H}_{K}$ are:

MP If $\vdash \varphi \rightarrow \psi$ and $\vdash \varphi$ then $\vdash \psi$,

$\mathrm{N} \quad$ If $\vdash \varphi$ then $\vdash \square \varphi$,

Namelite If $\vdash \neg i$ then $\vdash \perp$.

The additional rules of inference of $\mathcal{H}_{\mathrm{K}}^{+}$are:

Name $\quad$ If $\vdash i \rightarrow \varphi$ then $\vdash \varphi$, for $i$ not occurring in $\varphi$,

Paste $\quad$ If $\vdash \diamond^{n}(i \wedge \diamond(j \wedge \varphi)) \rightarrow \psi$ then $\vdash \diamond^{n}(i \wedge \diamond \varphi) \rightarrow \psi$ for $i \neq j$ and $j$ not occurring in $\varphi$ and $\psi$.

THEOREM 4.1 (Completeness ([24])). $\mathcal{H}_{K}$ is sound and strongly complete for the class of all descriptive two-sorted general frames.

Definition $4.8\left(\mathcal{H}_{K}(@)\right.$ axiomatization $\left.([14,24])\right)$. The axioms of $\mathcal{H}_{K}$ are all instances of the following schemata:

CT All classical tautologies, 


$$
\begin{array}{ll}
\mathrm{K} & \square(\varphi \rightarrow \psi) \rightarrow(\square \varphi \rightarrow \square \psi), \\
\mathrm{K}_{@} & @_{i}(\varphi \rightarrow \psi) \rightarrow\left(@_{i} \varphi \rightarrow @_{i} \psi\right), \\
\text { Ref } & @_{i} i, \\
\text { Intro } & i \wedge \varphi \rightarrow @_{i} \varphi, \\
\text { Back } & \diamond_{i} \varphi \rightarrow @_{i} \varphi, \\
\text { Agree } & @_{i} @_{j} \varphi \rightarrow @_{j} \varphi \\
\text { Back } & \bigotimes_{i} \varphi \rightarrow @_{i} \varphi .
\end{array}
$$

The rules of inference of $\mathcal{H}_{K}$ are:

MP If $\vdash \varphi \rightarrow \psi$ and $\vdash \varphi$ then $\vdash \psi$,

$\mathrm{N} \quad$ If $\vdash \varphi$ then $\vdash \square \varphi$,

$\mathrm{N}_{@} \quad$ If $\vdash \varphi$ then $\vdash @_{i} \varphi$ for $i \in$ NOM.

The additional rules of inference of $\mathcal{H}_{\mathrm{K}}^{+}$(@) are:

Name@ If $\vdash @_{i} \varphi$ then $\vdash \varphi$, for $i$ not occurring in $\varphi$,

BG If $\vdash @_{i} \diamond j \rightarrow @_{j} \varphi$ then $\vdash @_{i} \square \varphi$ for $i \neq j$ and $j$ not occurring in $\varphi$ and $\psi$.

TheOREM 4.2 (Completeness $([14,24])) . \mathcal{H}_{K}$ (@) is sound and strongly complete for the class of all descriptive two-sorted general frames.

Definition $4.9\left(\mathcal{H}_{K}(E)\right.$ axiomatization ([24])). The axioms of $\mathcal{H}_{K}$ are all instances of the following schemata:

CT All classical tautologies,

$\mathrm{K} \quad \square(\varphi \rightarrow \psi) \rightarrow(\square \varphi \rightarrow \square \psi)$,

$\mathrm{K}_{\mathrm{A}} \quad \mathrm{A}(\varphi \rightarrow \psi) \rightarrow(\mathrm{A} \varphi \rightarrow \mathrm{A} \psi)$,

$\operatorname{Ref}_{\mathrm{E}} \quad \varphi \rightarrow \mathrm{E} \varphi$,

Trans $_{\mathrm{E}} \mathrm{EE} \varphi \rightarrow \mathrm{E} \varphi$, 


$$
\begin{array}{ll}
\text { Sym }_{\mathrm{E}} & \varphi \rightarrow \operatorname{AE} \varphi, \\
\text { Incl }_{\diamond} & \diamond \varphi \rightarrow \mathrm{E} \varphi, \\
\text { Incl }_{i} & \mathrm{E} i,
\end{array}
$$

Nom $_{\mathrm{E}} \quad \mathrm{E}(i \wedge \varphi) \rightarrow \mathrm{A}(i \rightarrow \varphi$.

The rules of inference of $\mathcal{H}_{K}$ are:

MP If $\vdash \varphi \rightarrow \psi$ and $\vdash \varphi$ then $\vdash \psi$,

$\mathrm{N}_{\mathrm{A}} \quad$ If $\vdash \varphi$ then $\vdash \mathrm{A} \varphi$ for $i \in$ NOM.

The additional rules of inference of $\mathcal{H}_{\mathrm{K}}^{+}(\mathrm{E})$ are:

Name $\quad$ If $\vdash i \rightarrow \varphi$ then $\vdash \varphi$, for $i$ not occurring in $\varphi$,

$\mathrm{BG}_{\mathrm{E}} \quad$ If $\vdash \mathrm{E}(i \wedge \diamond j) \rightarrow \mathrm{E}(j \wedge \varphi)$ then $\mathrm{E}(i \wedge \square \varphi)$ for $i \neq j$ and $j$ not occurring in $\varphi$ and $\psi$.

THEOREM 4.3 (Completeness ([24])). $\mathcal{H}_{K}(E)$ is sound and strongly complete for the class of all descriptive two-sorted general frames.

THEOREM 4.4 ([24]). All inference rules from $\mathcal{H}_{\mathrm{K}}^{+}, \mathcal{H}_{\mathrm{K}}^{+}(@), \mathcal{H}_{\mathrm{K}}^{+}(E)$ preserve validity on strongly descriptive general frames.

All axioms and rules of inference follow rather straightforwardly from the semantic conditions given in Section 4.2 .

\subsection{COMPLEXITY}

In this section, we will provide a concise overview of the computational complexity of hybrid logics. Except for making several comments and quoting three theorems, we restrict ourselves to collating complexity results in Table 1.

Our first remark concerns the computational complexity of the hybrid logic $\mathcal{H}_{K}$ (@). It occurs that (up to a polynomial) we do not bear any computational costs when we augment the ordinary modal logic $K$ with nominals and the @ operators. The complexity class for $\mathcal{H}_{K}$ (@) remains PSPACE-complete, which is grasped by the following theorem: 


\begin{tabular}{l|c|c}
\multirow{2}{*}{ Frame class } & \multicolumn{2}{|c}{ Complexity class } \\
\cline { 2 - 3 } & $\mathcal{H}(@)$ & $\mathcal{H}(\mathrm{E})$ \\
\hline \hline arbitrary & PSPACE-complete & ExPTIME-complete \\
\hline reflexive & PSPACE-complete & ExPTIME-complete \\
\hline symmetric & PSPACE-complete & EXPTIME-complete \\
\hline serial & PSPACE-complete & EXPTIME-complete \\
\hline transitive & PSPACE-complete & PSPACE-complete \\
\hline equivalential & NP-complete & NP-complete
\end{tabular}

Table 1: Complexity classes for the logics $\mathcal{H}(@)$ and $\mathcal{H}(\mathrm{E})$ defined over various frame classes.

THEOREM $4.5([4,5])$. The satisfiability problem for the modal logic $K$ enriched with nominals and the @ operators is PSPACE-complete.

In most cases, hybrid logics inherit the complexity class after their modal counterparts. An interesting exception is the basic temporal logic. In this case, adding only one nominal to the basic temporal language suffices for the complexity to jump up from PSPACE-complete to EXPTIME-complete. The following theorem expresses this fact:

THEOREM $4.6([4,5])$. The satisfiability problem for the basic temporal logic enriched with at least one nominal is EXPTIME-complete.

Things get worse if we consider hybrid logics with binders. It appears that even $\mathcal{H}(\diamond, \downarrow)$, the weakest hybrid logic with binders with respect to the hierarchy presented in Section 4.3, yields the undecidable satisfiability problem, as stated in the following theorem:

THEOREM $4.7([5,12])$. The fragment of $\mathcal{H}_{K}(\diamond, \downarrow)$ consisting of all pure nominal-free sentences has an undecidable satisfiability problem.

3 The proof of NP-completeness of the logic $\mathcal{H}(\mathrm{E})$ over the class of equivalential frames consists in establishing the polysize model property for this logic. It exploits the fact that equivalence relations partition a universe into disjoint clusters. Euclidean relations divide a universe into quasi-clusters (clusters with at most one "tail point" not linked with all other members of a quasi-cluster). We therefore strongly conjecture that the proof of NP-completeness for $\mathcal{H}(@)$ and $\mathcal{H}(\mathrm{E})$ over equivalential frames can be extended on these logics defined over Euclidean frames. 
The proof of this fact consists in establishing the reduction of the unbounded tiling problem to the logic $\mathcal{H}(\downarrow, @)$. It exploits the spy-point technique. For details see $[12,13,5]$.

As it is shown in numerous papers by Schneider et al. $([85,69,66$, $68,38])$, restricting frame conditions usually leads to a drop of the complexity class, even for the logics with binders. In fact, for most frame classes they turn out to be decidable again! However, if we turn to the multi-modal case for hybrid logics with binders, they get back to the undecidable class. It is summarized by Mundhenk and Schneider in the fancy remark:

Remark 4.1 ([67]). If you hybridize the multi-modal logic $\mathcal{M}$, expect it to become undecidable - even if you only consider frame classes over which the uni-modal hybridized $\mathcal{M}$ is decidable.

By "hybridization" the authors of the remark mean the extension of a modal logic to a hybrid logic with at least one binder. Again, the result is based on encoding the unbounded tiling problem in a multi-modal hybrid logic with binders.

Finally, we present the complexity results for the hybrid logics taken into consideration in this book (see Table 1).

We do not consider the logic $\mathcal{H}(\mathrm{D})$ as a separate case since for every hybrid logic that contains nominals and the $E$ operator, adding D does not modify the complexity of its satisfiability problem ([4]).

\subsection{HISTORICAL OVERVIEW}

The history of hybrid logic can be traced back to the late 1960s when two collections of Arthur Prior's works on tense logic, Past, present and future ([80]) and Papers on time and tense ([81]), were published. Not digging deeply into details, it suffices to mention that Prior, while considering tense logic, introduced another sort of atomic expressions called by him possible world propositions, which can be regarded as a prototype of contemporary nominals. He also invented the binder $\forall$ to quantify over possible world propositions, and satisfaction statements 
$\mathrm{T}(a, \varphi)$ (expressing the fact that a formula $\varphi$ holds at a world $a^{4}$ ), which are denoted by @a $\varphi$ in the contemporary notation. This enriched tense logic constituted what Prior named third grade tense logic. In his fourth grade tense logic, he also introduced the operator known as the universal modality, which can be defined using satisfaction statements and $\forall$. As we can see, Prior's ideas have the full flavour of contemporary hybrid logic.

Prior's student, Robert Bull, introduced in [22] a third sort of expressions (except propositional variables and possible-world variables), namely path variables which one could also quantify over. His account was that in considerations on time flow not only concrete moments are significant but also time paths leading to that moment.

The next big step towards the systematic theory of hybrid logics was noticed in the mid 1980s due to the so-called "Sofia School of Modal Logic". In [77, 78], Passy and Tinchev provide a thorough exposition of the hybrid propositional dynamic logic. In [37], Gargov and Goranko deliver axiomatic and semantic characterization, and completeness results for the hybrid logic $\mathcal{H}(E)$. In [39], Goranko augmented the basic hybrid logic with the binder $\downarrow$.

The two decades from 1990 onwards can be considered as the most thriving period in the history of hybrid logics. Three major research areas at that time can be distinguished: the theory of hybrid languages, proof systems for hybrid logics, and the complexity aspects of these systems.

The first systematic characterisation of hybrid languages, their expressive hierarchy and interrelations (and, in fact, the first occurrence of the term "hybrid" in reference to modal logics with nominals), is delivered by Blackburn and Seligman in $[12,13]$. Further investigations concerning model theoretical properties of hybrid languages are performed in [5]. A comprehensive survey on the model theory for hybrid languages can be found in ten Cate's [24].

In [88], Seligman introduces a sequent calculus for the hybrid logic $\mathcal{H}(\downarrow, \exists$, @ ), preceded by his calculi exhibited in [86, 87]. An internalized tableau calculus for $\mathcal{H}(@)$ is proposed by Blackburn in [10], whereas

4 The very same idea can be found in James Allen's work [2], where he introduces the operator Holds acting in the same way as Prior's T. 
Tzakova establishes a prefixed tableau calculus for $\mathcal{H}(\downarrow, \exists, @)$ in [93] (however, the latter system contains a flaw in the termination proof, detected by Bolander and Braüner in [18]). Later works on the proof theory for hybrid logics are mostly due to Blackburn, Bolander and Braüner ([20, 18, 19, 16, 17], and also by Kaminski, Smolka et al. ([56, 58]). An extensive study of proof systems for hybrid logics is given by Braüner [21].

The computational complexity of hybrid logics with binders was firstly investigated by Blackburn and Seligman in [12, 13]. First complexity results for binder-free hybrid logics were established by Areces, Blackburn and Marx in [3,4,5]. An exhaustive analysis of the computational complexity of most hybrid logics defined over various frame classes was provided in numerous works $([85,69,66,68,38])$ by Schneider, Mundhenk et al.

One cannot forget about contributions by the description logics community to the development of hybrid logics. Most works concerning the automated reasoning in description logics involve nominals which occur explicitly as formulas whose interpretations are singleton sets, or under the name of individuals as entities occurring in so-called $A B o x$ s $^{5}$. To get the flavour of the description logic approach to the hybrid logic tools, the reader is recommended to get acquainted with $[49,46,51,47]$.

More detailed and comprehensive discussion on the history of hybrid logic can be found in works of Blackburn $([9,11])$, Braüner ([21]) and Indrzejczak ([52]).

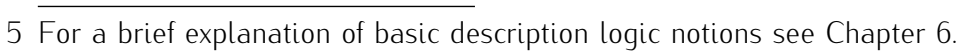




\section{$\operatorname{LOGIC} \mathcal{M}\left(\mathrm{E}_{n}\right)$}

The present chapter is more extensive than the previous one since the reported results are mostly new. They previously occurred in two papers by the author of the book: [102] and [104]

\subsection{MOTIVATION}

Ordinary modal logics do not distinguish between worlds satisfying the same formulas. It means that whenever a modal formula $\varphi$ is satisfied by a model $\mathfrak{M}$, it is also satisfied by a model $\mathfrak{M}^{\prime}$ which collapses identical states from $\mathfrak{M}^{\prime}$ to only one state. If we reason about concrete domains represented by modal models and we want to express the fact that a particular formula (representing, e.g., a particular state of affairs) occurs in a domain, we need to augment the ordinary modal language with some additional operators. Introducing graded modalities of the form $\nabla_{>n}, \nabla_{<n}, \nabla_{=n}$ allowed to express the cardinality of a set of a particular world's successors that satisfy a certain formula $\varphi$. However, graded modalities do not enable making global numerical restrictions. Only introducing global counting operators of the form $E_{>n}, E_{<n}$, and $\mathrm{E}_{=n}$ fills this gap. The syntax and semantics of a language enriched with global counting modalities is presented in Section 5.2. In Section 5.3, it is shown that taking into account modal logics with global counting operators in the book devoted to hybrid logics is not a whim and that it is fully justified by the expressive power of such logics.

1 Section 5.4 is reprinted from "Bulletin of the Section of Logic", Vol. 41(3-4), Michat Zawidzki, Adequacy of the logic $\mathrm{K}\left(\mathrm{E}_{n}\right)$, Pages No. 155-172, Copyright (2012), with permission from Łódź University Press ([102]).

The part of Section 5.5 on pages 57-64 is reprinted from "Information Processing Letters", Vol. 113(1-2), Michat Zawidzki, Renate A. Schmidt, Dmitry Tishkovsky, Satisfiability problem for modal logic with global counting operators coded in binary is NEXPTIME-complete, Pages No. 34-38, Copyright (2013), with permission from Elsevier ([104]). 
A few words of comment should be devoted to the problem of coding numerical subscripts in counting operators. Subscript $n$ coded in unary can be regarded as a sequence of $n$ consecutive $1 \mathrm{~s}$. The size of this sequence equals $n$. Subscript $n$ coded in binary is a sequence of consecutive bits which can take values from the set $\{0,1\}$, whose binary value equals $n$. The size of such a sequence equals $\log (n)^{2}$. In this book we make the binary coding assumption since it is more natural and better motivated by practical applications of decision procedures for this logic.

Henceforth, the modal logic with global counting operators is abbreviated as $\mathcal{M}\left(\mathrm{E}_{n}\right)^{3}$.

\subsection{SYNTAX AND SEMANTICS}

First, we need to define the language of $\mathcal{M}\left(\mathrm{E}_{n}\right)$.

Definition 5.1 (Syntax). Let PROP $=\left\{p_{1}, p_{2}, \ldots\right\}$ be a countable set of propositional letters. We define a set Form of formulas of $\mathcal{M}\left(E_{n}\right)$ as follows:

$$
\text { FORM }:=p|\neg \varphi| \varphi \wedge \psi|\diamond \varphi| \mathrm{E}_{>n} \varphi,
$$

where $p \in \operatorname{PROP}, \varphi, \psi \in$ FORM, $n \in \mathbb{N}$. We also give explicit definitions of other Boolean connectives and modal operators.

$$
\begin{array}{ll}
\varphi \vee \psi:=\neg(\neg \varphi \wedge \neg \psi), & \mathrm{E}_{<n} \varphi:=\neg \mathrm{E}_{>n-1} \varphi, \\
\varphi \rightarrow \psi:=\neg(\varphi \wedge \neg \psi), & \mathrm{E}_{=n+1} \varphi:=\mathrm{E}_{>n} \varphi \wedge \neg \mathrm{E}_{>n+1} \varphi, \\
\square \varphi:=\neg \diamond \neg \varphi, & \mathrm{E}_{=0} \varphi:=\neg \mathrm{E}_{>0} \varphi .
\end{array}
$$

The operators $\nabla_{<n}$ and $\nabla_{=n}$ are defined analogously to $\mathrm{E}_{<n}$ and $\mathrm{E}_{=n}$. Moreover, note that $E_{>0}$ and $\nabla_{>0}$ stand for, respectively, the ordinary global modality and the ordinary $\diamond$ modality.

2 Since we mostly discuss powers and logarithms with 2 as a base, by $\log (n)$ we denote $\log _{2}(n)$

3 Whenever we refer to a concrete modal logic augmented with global counting modalities, we add a subscript referring to the name of this logic. For instance, if we consider the logic $K$ with global counting operators, we denote it by $\mathcal{M}_{K}\left(E_{n}\right)$. 
Definition 5.2 (Semantics). A model for $\mathcal{M}\left(\mathrm{E}_{n}\right)$ is a triple $\langle W, R, V\rangle$ where $W$ is a non-empty set, $R$ is a binary relation on $W$, $V: \mathrm{PROP} \rightarrow \mathcal{P}(W)$ is a valuation function assigning to each $p \in \mathrm{PROP}$ a set of worlds $w \in W$ in which $p$ holds. Given a model $\langle W, R, V\rangle$ and $w \in W$, the semantics for $\mathcal{M}\left(\mathrm{E}_{n}\right)$ is defined as follows:

$$
\begin{array}{lll}
\mathfrak{M}, w \Vdash p & \text { iff } & w \in V(p), p \in \mathrm{PROP}, \\
\mathfrak{M}, w \Vdash \neg \varphi & \text { iff } & \mathfrak{M}, w \Vdash \varphi, \\
\mathfrak{M}, w \Vdash \varphi \wedge \psi & \text { iff } & \mathfrak{M}, w \Vdash \varphi \text { and } \mathfrak{M}, w \Vdash \psi, \\
\mathfrak{M}, w \Vdash \triangleright \varphi & \text { iff } & \text { there exists } v \text { such that } w R v \text { and } \mathfrak{M}, v \Vdash \varphi, \\
\mathfrak{M}, w \Vdash \mathrm{E}_{>n} \varphi & \text { iff } & \operatorname{Card}(\{v \mid \mathfrak{M}, v \Vdash \varphi\})>n,
\end{array}
$$

where $\operatorname{Card}(A)$ denotes the cardinality of the set $A$.

\section{$5 \cdot 3$ EXPRESSIVITY}

But why should we bother with $\mathcal{M}\left(\mathrm{E}_{n}\right)$ at all? - one might ask. Well, it turns out that its expressive power justifies our interest. Not only are we able to encode the size of a model in a formula of the form:

$$
\mathrm{E}_{=n} \top .
$$

What is more important, global counting operators enable us to gain the full expressive power of hybrid language with the universal modality, the difference modality and the satisfaction operators.

In order to demonstrate it, we introduce the following translation function: $\operatorname{Tr}_{\mathrm{E}_{n}}: \mathcal{H}(\mathrm{E}, \mathrm{D}, @) \longrightarrow \mathcal{M}\left(\mathrm{E}_{n}\right)$ :

$$
\begin{array}{ll}
\operatorname{Tr}_{\mathrm{E}_{n}}(p) & =p, \\
\operatorname{Tr}_{\mathrm{E}_{n}}(i) & =\mathrm{E}_{=1} p_{i} \wedge p_{i}, \\
\operatorname{Tr}_{\mathrm{E}_{n}}(\neg \varphi) & =\neg \operatorname{Tr}_{\mathrm{E}_{n}}(\varphi), \\
\operatorname{Tr}_{\mathrm{E}_{n}}(\varphi \wedge \psi) & =\operatorname{Tr}_{\mathrm{E}_{n}}(\varphi) \wedge \operatorname{Tr}_{\mathrm{E}_{n}}(\psi), \\
\operatorname{Tr}_{\mathrm{E}_{n}}(\diamond \varphi) & =\diamond \operatorname{Tr}_{\mathrm{E}_{n}}(\varphi), \\
\operatorname{Tr}_{\mathrm{E}_{n}}(@) & =\mathrm{E}_{=1} p_{i} \wedge \mathrm{E}_{>0}\left(p_{i} \wedge \operatorname{Tr}_{\mathrm{E}_{n}}(\varphi)\right), \\
\operatorname{Tr}_{\mathrm{E}_{n}}(\mathrm{E} \varphi) & =\mathrm{E}_{>0}\left(\operatorname{Tr}_{\mathrm{E}_{n}}(\varphi)\right), \\
\operatorname{Tr}_{\mathrm{E}_{n}}(\mathrm{D} \varphi) & =\left(\operatorname{Tr}_{\mathrm{E}_{n}}(\varphi) \wedge \mathrm{E}_{>1}\left(\operatorname{Tr}_{\mathrm{E}_{n}}(\varphi)\right)\right) \vee\left(\neg \operatorname{Tr}_{\mathrm{E}_{n}}(\varphi) \wedge \mathrm{E}_{>0}\left(\operatorname{Tr}_{\mathrm{E}_{n}}(\varphi)\right)\right),
\end{array}
$$


where $p_{i}$ is a fresh propositional variable not occurring in $\varphi$ and $V$ is defined in a standard way. Note that whenever a hybrid formula $\varphi$ is satisfiable on $\mathfrak{M}, \operatorname{Tr}_{\mathrm{E}_{n}}(\varphi)$ is satisfiable on $\mathfrak{M}^{\prime}$, where $\mathfrak{M}^{\prime}$ is a conservative extension of $\mathfrak{M}$ where we only add $p_{1}, \ldots, p_{n}$ introduced by $\operatorname{Tr}_{\mathrm{E}_{n}}$ to the signature. As we can see, we can consider $\mathcal{M}\left(E_{n}\right)$ as a nonclassical variant of hybrid logic! The translation function $\operatorname{Tr}_{E_{n}}$ is linear in the size of a translated formula. As we can find in [6], there also exists a reverse translation function, let's call it $\operatorname{Tr}_{\mathcal{H}(E)}$. It is defined as follows:

$$
\begin{aligned}
\operatorname{Tr}_{\mathcal{H}(\mathrm{E})}(p) & =p, \\
\operatorname{Tr}_{\mathcal{H}(\mathrm{E})} & =\neg \operatorname{Tr}_{\mathcal{H}(\mathrm{E})}(\varphi), \\
\operatorname{Tr}_{\mathcal{H}(\mathrm{E})}(\varphi \wedge \psi) & =\operatorname{Tr}_{\mathcal{H}(\mathrm{E})}(\varphi) \wedge \operatorname{Tr}_{\mathcal{H}(\mathrm{E})}(\psi), \\
\operatorname{Tr}_{\mathcal{H}(\mathrm{E})}(\diamond \varphi) & =\diamond \operatorname{Tr}_{\mathcal{H}(\mathrm{E})}(\varphi), \\
\operatorname{Tr}_{\mathcal{H}(\mathrm{E})}\left(\mathrm{E}_{>n} \varphi\right) & =\bigwedge_{1 \leq k<l \leq n+1} @_{i_{k}} \neg i_{l} \wedge \bigwedge_{1 \leq k \leq n+1} @_{i_{k}}\left(\operatorname{Tr}_{\mathcal{H}(\mathrm{E})}(\varphi)\right), \\
\operatorname{Tr}_{\mathcal{H}(\mathrm{E})}\left(\mathrm{E}_{<n} \varphi\right) & =\mathrm{A}\left(\neg \operatorname{Tr}_{\mathcal{H}(\mathrm{E})}(\varphi) \vee \bigvee_{1 \leq k \leq n-1} i_{k}\right),
\end{aligned}
$$

where $i_{1}, \ldots i_{n+1}$ are freshly introduced nominals, $V$ and $A$ are defined in a standard way. However, if we assume that numbers in $\mathcal{M}\left(\mathrm{E}_{n}\right)$ are coded in binary, $\operatorname{Tr}_{\mathcal{H}(\mathrm{E})}(\varphi)$ is exponentially larger than $\varphi$ whenever numbers occur in $\varphi$.

Henceforth, for the reasons given above, we consider $\mathcal{M}\left(\mathrm{E}_{n}\right)$ as a hybrid logic and present it as such in the book.

\subsection{HILBERT-STyLE CALCULUS For $\mathcal{M}_{K}\left(E_{n}\right)$}

In this section we provide a sound and complete axiomatization for $\mathcal{M}_{K}\left(E_{n}\right)$ and by that means give rise to a Hilbert-style calculus for this logic.

\subsubsection{Axiomatization for $\mathcal{M}_{K}\left(\mathrm{E}_{n}\right)$}

Now we give axiomatization for the logic $\mathcal{M}_{K}\left(E_{n}\right)$.

Definition $5.3\left(\mathcal{M}_{K}\left(E_{n}\right)\right.$ axiomatization). The axioms of $\mathcal{M}_{K}\left(E_{n}\right)$ are all instances of the following schemata: 
CT All substitutions of classical tautologies,

$\mathrm{K} \quad \square(\varphi \rightarrow \psi) \rightarrow(\square \varphi \rightarrow \square \psi)$,

$\mathrm{K}_{\mathrm{E}_{n}} \quad \mathrm{E}_{=0} \neg(\varphi \rightarrow \psi) \rightarrow\left(\mathrm{E}_{>n} \varphi \rightarrow \mathrm{E}_{>n} \psi\right)$, for $n \in \mathbb{N}$,

Disj $\mathrm{E}_{=0}(\varphi \wedge \psi) \rightarrow\left(\left(\mathrm{E}_{=n} \varphi \wedge \mathrm{E}_{=m} \psi\right) \rightarrow \mathrm{E}_{=n+m}(\varphi \vee \psi)\right)$, for $n, m \in \mathbb{N}$,

$\operatorname{lnt}_{\mathrm{E}_{n}} \quad \varphi \rightarrow \mathrm{E}_{>0} \varphi$,

$\operatorname{Tot}_{\mathrm{E}_{n}} \quad \mathrm{E}_{>n} \varphi \rightarrow \mathrm{E}_{=0}\left(\neg \mathrm{E}_{>n} \varphi\right)$, for $n \in \mathbb{N}$,

Num $\quad \mathrm{E}_{>n} \varphi \rightarrow \mathrm{E}_{>m} \varphi$, for $n, m \in \mathbb{N}, n>m$,

$\operatorname{Tot} 2 \mathrm{E}_{n} \quad \diamond \varphi \rightarrow \mathrm{E}_{>0} \varphi$.

The rules of inference of $\mathcal{M}_{K}\left(E_{n}\right)$ are:

MP If $\vdash \varphi \rightarrow \psi$ and $\vdash \varphi$ then $\vdash \psi$,

$\mathrm{N}_{\mathrm{E}} \quad$ If $\vdash \varphi$ then $\vdash \mathrm{E}_{=0} \neg \varphi$.

Note that the ordinary necessitation rule of the form "If $\vdash \varphi$ then

$\vdash \square \varphi^{\prime \prime}$ can be derived from $\mathrm{N}_{\mathrm{E}}$, Tot $2 \mathrm{E}_{n}$ and from the fact that $\mathrm{E}_{=0} \varphi:=$ $\neg \mathrm{E}_{>0} \varphi$.

5.4.2 Adequacy of $\mathcal{M}_{K}\left(E_{n}\right)$

We now prove that $\mathcal{M}_{K}\left(E_{n}\right)$ is complete with respect to the class of all frames. We proceed in two steps. First comes the proof of the easy part.

\section{Soundness}

THEOREM 5.1. The axiomatization from Definition 5.3 is sound with respect to the class of all frames, i.e. if $\vdash \mathcal{M}_{K}\left(\mathrm{E}_{n}\right) \varphi$ then $\mid=\mathcal{M}_{K}\left(\mathrm{E}_{n}\right) \varphi$.

Proof. We prove the theorem by checking validity of the axioms and testing whether the rules of inference preserve validity. For obvious reasons we skip the proof for $C T$, axiom $\mathrm{K}$ and for the rule MP.

Let $\mathfrak{F}=\langle W, R$, $\rangle$ be arbitrary frame, $V$ arbitrary valuation function, $\checkmark$ arbitrary world and $\mathfrak{M}=\langle\mathfrak{F}, V\rangle$. 
$\mathrm{K}_{\mathrm{E}_{n}}$ Assume that $\mathfrak{M}, v \Vdash \mathrm{E}_{=0} \neg(\varphi \rightarrow \psi)$ and $\mathfrak{M}, v \Vdash \mathrm{E}_{>n} \varphi$. By definition of $\mathrm{E}_{=0}$ we have that $\operatorname{Card}(\{w \mid \mathfrak{M}, w \Vdash \varphi\}) \leq 0$. Let $W^{\prime}$ be a set of all worlds satisfying $\varphi$. We know that $\operatorname{Card}\left(W^{\prime}\right)>n$. We also know that $\varphi \rightarrow \psi$ holds at all worlds $w \in W \supseteq W^{\prime}$, hence $\varphi \rightarrow \psi$ is satisfied by all worlds $w \in W^{\prime}$. But $\operatorname{Card}\left(W^{\prime}\right)>n$, whence, by $\mathrm{MP}, \mathfrak{M}, v \Vdash \mathrm{E}_{>n} \psi$.

Disj Assume that $\mathfrak{M}, v \Vdash \mathrm{E}_{=0}(\varphi \wedge \psi)$ and $\mathfrak{M}, v \Vdash \mathrm{E}_{=n} \varphi \wedge \mathrm{E}_{=m} \psi$. From the first part of the assumption we have that $\neg E_{>0}(\varphi \wedge \psi)$, whence, by definition of $\mathrm{E}_{=0}$, we get $\operatorname{Card}(\{w \mid \mathfrak{M}, w \Vdash \varphi \wedge \psi\}) \leq 0$ and what follows, $\operatorname{Card}(\{w \mid \mathfrak{M}, w \Vdash \neg(\varphi \wedge \psi)\})=\operatorname{Card}(W)$. So there is no such world $w$ that $\mathfrak{M}, w \Vdash \varphi \wedge \psi$. Now, the second part of the assumption says that $\mathrm{E}_{=n} \varphi \wedge \mathrm{E}_{=m} \psi$. In other words $\{w \mid$ $\mathfrak{M}, w \Vdash \varphi\} \cap\{w \mid \mathfrak{M}, w \Vdash \psi\}=\varnothing$, so, by elementary facts of set theory, we have $\operatorname{Card}(\{w \mid \mathfrak{M}, w \Vdash \varphi\} \cup\{w \mid \mathfrak{M}, w \Vdash \psi\})=$ $\operatorname{Card}(\{w \mid \mathfrak{M}, w \Vdash \varphi$ or $\mathfrak{M}, w \Vdash \psi\})=\operatorname{Card}(\{w \mid \mathfrak{M}, w \Vdash \varphi \vee$ $\psi\})=n+m$. Hence $\mathfrak{M}, v \Vdash \mathrm{E}_{=n+m}(\varphi \vee \psi)$.

$\operatorname{Int}_{\mathrm{E}_{n}}$ Assume that $\mathfrak{M}, v \Vdash \varphi$. Then there is at least one world in $W$ in which $\varphi$ holds. Therefore $\operatorname{Card}(\{w \mid \mathfrak{M}, w \Vdash \varphi\})>0$, whence $\mathfrak{M}, v \Vdash \mathrm{E}_{>0} \varphi$.

Tot $1_{\mathrm{E}_{n}}$ Assume that $\mathfrak{M}, v \Vdash \mathrm{E}_{>n} \varphi$. We have then that $\operatorname{Card}(\{w \mid$ $\mathfrak{M}, w \Vdash \varphi\})>n$. Obviously, by semantic definition of $\mathbf{E}_{>n}$ we have $\mathfrak{M}, v \Vdash \mathbf{E}_{>n} \varphi$ for every $v \in W$. Since $\left\{w \mid \mathfrak{M}, w \Vdash \mathbf{E}_{>n} \varphi\right\}=W$ we have that $U=\left\{w \mid \mathfrak{M}, w \Vdash \neg \mathrm{E}_{>n} \varphi\right\}=\varnothing$, so $\operatorname{Card}(U)=0$. Hence $\mathfrak{M}, v \Vdash \mathrm{E}_{=0}\left(\neg \mathrm{E}_{>n} \varphi\right)$.

Num Assume that $\mathfrak{M}, v \Vdash \mathrm{E}_{>n} \varphi$. Hence $\operatorname{Card}(\{w \mid \mathfrak{M}, w \Vdash \varphi\})>$ $n$. But if $m<n$, then obviously $\operatorname{Card}(\{w \mid \mathfrak{M}, w \Vdash \varphi\})>m$. Consequently, $\mathfrak{M}, v \Vdash \mathrm{E}_{>m} \varphi$.

Tot $2 \mathrm{E}_{n}$ Assume that $\mathfrak{M}, v \Vdash \diamond \varphi$. Then there is such a world $w \in W$ that $v R w$ and $\mathfrak{M}, w \Vdash \varphi$. Hence follows that there is at least one world $w \in W$ in which $\varphi$ holds, i.e. $\operatorname{Card}(\{w \mid \mathfrak{M}, w \Vdash \varphi\})>0$, whence we obtain $\mathfrak{M}, v \Vdash \mathrm{E}_{>0} \varphi$.

$\mathrm{N}_{\mathrm{E}}$. Assume that $\mathfrak{M} \Vdash \varphi$. Then $\varphi$ holds at all worlds $v \in W$ whence $U=\{w \mid \mathfrak{M}, w \Vdash \neg \varphi\}=\varnothing$, so $\operatorname{Card}(U)=0$ and, by definition of $\mathrm{E}_{=n}, \mathfrak{M} \Vdash \mathrm{E}_{=0} \neg \varphi$. 
Since $\mathfrak{F}$ was an arbitrary frame, $V$ and $v$ were arbitrary, the theorem holds.

\section{Completeness}

PRELIMINARIES Proof of the second part of the adequacy theorem, namely the completeness theorem, is, as usual, much more complicated. To establish it, we will first need the following fact:

FACT 5.2. A logic $\mathcal{L}$ is strongly complete with respect to a class of frames $\mathcal{F}$ iff every $\mathcal{L}$-consistent set of formulas is satisfiable on some $\mathfrak{F} \in \mathcal{F}$.

The above fact allows us to conduct a proof by constructing a model. Namely, we show that every $\mathcal{M}_{K}\left(E_{n}\right)$-consistent set is satisfiable in a model of the class of all frames. A major part of the proof and preliminary lemmas come from [32] and, in a simplified and more general form, from [23]. Fattorosi-Barnaba and Caro proved completeness for several normal modal logics with graded modalities. We can use their results for the "counting" part of our work and recall them in the book for the purpose of clarity of the argument.

We start with several introductory lemmas concerning properties of $\mathcal{M}_{K}\left(E_{n}\right)$-maximal consistent sets (MCS). First, it appears to be useful to generalize Disj.

LEMMA 5.3. Let $\varphi_{1}, \ldots, \varphi_{k} \in$ FORM. If $\vdash \bigwedge_{i, j \leq k}^{i \neq j} \neg\left(\varphi_{i} \wedge \varphi_{j}\right)$, then $\vdash \bigwedge_{i \leq k} \mathrm{E}_{=n_{i}} \varphi_{i} \rightarrow \mathrm{E}_{=\sum_{i=1}^{k} n_{i}} \bigvee_{i \leq k} \varphi_{i}, n_{i} \in \mathbb{N}$.

Proof. By induction on $k$.

BASE CASE: $k=1$. We have $\vdash \neg\left(\varphi_{0} \wedge \varphi_{1}\right)$. Hence, by $N_{\mathrm{E}}$, we obtain $\vdash \mathrm{E}_{=0}\left(\varphi_{0} \wedge \varphi_{1}\right)$ and by Disj we get the conclusion: $\mathrm{E}_{=n_{0}} \varphi_{0} \wedge \mathrm{E}_{=n_{1}} \varphi_{1} \rightarrow$ $\mathrm{E}_{=n_{0}+n_{1}}\left(\varphi_{0} \vee \varphi_{1}\right)$.

INDUCTIVE STEP: Suppose that the conclusion holds for $k$. $\vdash \bigwedge_{i, j \leq k}^{i \neq j} \neg\left(\varphi_{i} \wedge \varphi_{j}\right) \quad$ iff $\vdash \quad \bigwedge_{i, j \leq k}^{i \neq j} \neg\left(\varphi_{i} \wedge \varphi_{j}\right) \wedge \bigwedge_{i \leq k} \neg\left(\varphi_{i} \wedge \varphi_{k+1}\right)$. By the inductive hypothesis and base case we obtain: $\vdash \bigwedge_{i \leq k+1} \mathrm{E}_{=n_{i}} \varphi_{i} \rightarrow \mathrm{E}_{=\sum_{i=1}^{k} n_{i}} \bigvee_{i \leq k} \varphi_{i} \wedge \mathrm{E}_{=n_{k+1}} \varphi_{k+1}$ By $\quad \mathrm{CT}, \vdash \quad \bigwedge_{i \leq k} \neg\left(\varphi_{i} \wedge \varphi_{k+1}\right) \quad$ iff $\vdash \quad \neg \bigvee_{i \leq k}\left(\varphi_{i} \wedge \varphi_{k+1}\right)$ iff $\quad \vdash \quad \neg\left(\bigvee_{i \leq k}\left(\varphi_{i}\right) \wedge \varphi_{k+1}\right)$, so we obtain 
$\vdash \mathrm{E}_{=\sum_{i=1}^{k} n_{i}} \mathrm{~V}_{i \leq k}\left(\varphi_{i}\right) \wedge \mathrm{E}_{=n_{k+1}} \varphi_{k+1} \rightarrow \mathrm{E}_{\sum_{i=1}^{k+1} n_{i}} \mathrm{~V}_{i \leq k+1} \varphi_{i}$. Hence, we have $\vdash \bigwedge_{i \leq k+1} \mathrm{E}_{=n_{i}} \varphi_{i} \rightarrow \mathrm{E}_{=\sum_{i=1}^{k+1} n_{i}} \bigvee_{i \leq k+1} \varphi_{i}$

Therefore, by the proof of the base and the inductive step, the conclusion follows for every finite $k$.

LEMMA 5.4. Let $\Gamma$ be a MCS of formulas of $\mathcal{M}_{K}\left(E_{n}\right)$. We have:

1. If $\mathrm{E}_{=n} \varphi \in \Gamma$ then $\mathrm{E}_{=m} \varphi \notin \Gamma$ for all $m \in \mathbb{N}$ such that $n \neq m$.

2. For every $\varphi \in$ FORM exactly one of the following conditions holds:

a. for every $n \in \mathbb{N}: \mathbb{E}_{>n} \varphi \in \Gamma$,

b. there is $n \in \mathbb{N}$ such that $\mathrm{E}_{=n} \varphi \in \Gamma$.

3. If $\vdash \varphi \rightarrow \psi$ and $\mathrm{E}_{=n} \psi \in \Gamma$, then there is $m \in \mathbb{N}$ such that $\mathrm{E}_{=m} \varphi \in \Gamma$ and necessarily $m \leq n$.

Proof. 1. Suppose conversely that there is such a formula $\varphi$ that $\mathrm{E}_{=n} \varphi \in \Gamma, \mathrm{E}_{=m} \varphi \in \Gamma$ and $n \neq m$. Suppose without loss of generality that $n>m$. We have then that $n-1 \geq m$ and, by definition of $\mathrm{E}_{=n}$ and maximality of $\Gamma$, that $\mathrm{E}_{>n-1} \varphi \in \Gamma$ and thus that $\mathrm{E}_{>m} \varphi \in \Gamma$. But we also have that $\mathrm{E}_{>m} \varphi \notin \Gamma$. Contradiction.

2. Both conditions are complementary. Suppose that a. does not hold. Then it is the case that there is such $n \in \mathbb{N}$ that $\mathrm{E}_{>_{n}} \varphi \notin \Gamma$. Let $n_{0}$ be the least such $n$. We have that $\mathrm{E}_{>n_{0}} \varphi \notin \Gamma$, so $\neg \mathrm{E}_{>n_{0}} \varphi \in \Gamma$. By the assumption of minimality of $n_{0}$ we also have that $\mathrm{E}_{>n_{0}-1} \varphi \in \Gamma$. Thus we obtain $\mathrm{E}_{=n_{0}} \varphi \in \Gamma$. By 1. we get uniqueness of $n_{0}$. Now suppose that a. holds. Then for all $n \in \mathbb{N}_{>n} \varphi \in \Gamma$. By the consistency of $\Gamma$ for all $n \in \mathbb{N} \neg \mathrm{E}_{>n} \varphi \notin \Gamma$, therefore by the definition of $\mathrm{E}_{=n}$ there exists no $n \in \mathbb{N}$ such that $\mathrm{E}_{=n} \varphi \in \Gamma$.

3. Suppose that $\vdash \varphi \rightarrow \psi$ and $\mathrm{E}_{=n} \psi \in \Gamma$. By the monotonicity of $\mathrm{E}_{>n}$ with respect to implication we obtain $\vdash \mathrm{E}_{>n} \varphi \rightarrow \mathrm{E}_{>n} \psi$ and by definition of $\mathrm{E}_{=n}$ we have that $\neg \mathrm{E}_{>n} \psi \in \Gamma$. Therefore, $\neg \mathrm{E}_{>n} \varphi \in \Gamma$ and by Num $\neg \mathrm{E}_{>m} \varphi \in \Gamma$ for all $m>n\left(\neg \mathrm{E}_{>n} \varphi=\mathrm{E}_{<n+1} \varphi\right)$. By 2. it follows that there is $m \in \mathbb{N}$ such that $\mathrm{E}_{=m} \varphi \in \Gamma$. By the definition of $\mathrm{E}_{=n} m$ is not greater than $n$, so we have $\mathrm{E}_{=m} \varphi \in \Gamma, m \leq n$. 
LemMA 5.5. Let $\Gamma$ be a MCS and $\varphi_{0}, \ldots, \varphi_{k}$ - formulas of $\mathcal{M}_{K}\left(\mathrm{E}_{n}\right)$. Let $\psi_{i}$ be a formula of the form $\varphi_{0} \wedge \bar{\varphi}_{1} \wedge \cdots \wedge \bar{\varphi}_{k}$ where $\bar{\varphi}_{i}=\varphi_{i}$ or $\neg \varphi_{i}$. Then we have a set of distinct formulas $\left\{\psi_{i} \mid 0 \leq i<2^{k}\right\}$.

1. If there is such $n \in \mathbb{N}$ that $\mathrm{E}_{=n} \varphi_{0} \in \Gamma$ then there are also $n_{0}, \ldots, n_{2^{k}-1}$ such that $\sum_{i=0}^{2^{k}-1} n_{i}=n$ and $\mathrm{E}_{=n_{i}} \psi_{i} \in \Gamma,\left(0 \leq i<2^{k}\right)$.

2. If $\mathrm{E}_{>n} \varphi_{0} \in \Gamma$ for all $n \in \mathbb{N}$ then there is such $i, 0 \leq i<2^{k}$ that $\mathrm{E}_{>n} \psi \in \Gamma$ for all $n \in \mathbb{N}$.

Proof. 1. Assume that $\mathrm{E}_{=n} \varphi_{0} \in \Gamma$. By CT we have that $\psi_{i} \neq \psi_{j}$ for $i \neq j, 0 \leq i, j<2^{k}$. We show that $\vdash \varphi_{0} \leftrightarrow \bigvee_{i<2^{k}} \psi_{i}$. By the generalized law of excluded middle we have that $\vdash \bigvee_{i<2^{k}} \psi_{i}^{\prime} \leftrightarrow T$ where $\psi_{i}^{\prime}$ is a formula obtained from $\psi_{i}$ by deleting $\varphi_{0}$. Then we have $\vdash \bigvee_{i<2^{k}} \psi_{i} \leftrightarrow \varphi_{0} \wedge \bigvee_{i<2^{k}} \psi_{i}^{\prime} \leftrightarrow \varphi_{0} \wedge T \leftrightarrow \varphi_{0}$. Since $\vdash \psi_{i} \rightarrow \varphi_{0}$ for all $0 \leq i<2^{k}$, by Lemma 5.4 we have $\mathrm{E}_{=m_{1}} \psi_{i} \in \Gamma$ such that $m_{i} \leq n$. Then by Lemma 5.3 and the monotonicity of $E_{=n}$ with respect to equivalence we have that there is a set of indices $\left\{n_{i} \mid 0 \leq i<2^{k}\right\}$ such that $\mathrm{E}_{=n_{i}} \psi_{i}$ and $\sum_{i=0}^{2^{k}-1} n_{i}=n$.

2. Suppose conversely that there is a set of indices $\left\{n_{i} \mid 0 \leq i<2^{k}\right\}$ such that $\mathrm{E}_{=n_{i}} \psi_{i} \in \Gamma$ for $0 \leq i<2^{k}$. By 1. we have that $\mathrm{E}_{=n} \varphi_{0}$ for a certain $n \in \mathbb{N}$. But by the assumption, $\mathrm{E}_{>m} \in \Gamma$ for all $m \in \mathbb{N}$, so in particular $\mathrm{E}_{>n} \in \Gamma$. Contradiction.

We can now turn to the proper part of our work, namely the construction of a model that will satisfy arbitrarily chosen $\mathcal{M}_{K}\left(E_{n}\right)$-consistent set of formulas. Let $\Gamma^{-}$be our $\mathcal{M}_{K}\left(E_{n}\right)$-consistent set of formulas. Let us introduce a following notation:

Notation. We will denote a family of all $\mathcal{M}_{\mathrm{K}}\left(\mathrm{E}_{n}\right)-\mathrm{MCS}$ by $\mathfrak{G}$.

By Lindenbaum's Lemma there exists a $\mathcal{M}_{K}\left(E_{n}\right)-M C S$, name it $\Gamma$, that includes $\Gamma^{-}$(in fact, if $\Gamma^{-}$is finite then there are infinitely many such MCSs. We can arbitrarily choose one). Obviously $\Gamma \in \mathfrak{G}$.

We define a function that will help us set the universe of our intended model. 
Definition 5.4. Let $\Gamma_{1}, \Gamma_{2} \in \mathfrak{G}$. The function $\lambda: \mathfrak{G} \times \mathfrak{G} \longrightarrow \mathbb{N} \cup\{\omega\}$ is defined as follows $\lambda\left(\Gamma_{1}, \Gamma_{2}\right)=\left\{\begin{array}{l}\omega, \text { if for any } \varphi \in \Gamma_{2}, \mathrm{E}_{>n} \varphi \in \Gamma_{1} \text { for any } n \in \mathbb{N} \\ h=\min \left\{n \in \mathbb{N} \mid \mathrm{E}_{=n} \varphi \in \Gamma_{1} \text { and } \varphi \in \Gamma_{2}\right\}, \text { otherwise. }\end{array}\right.$

The above function is well defined. For if it is not the case that for any $\varphi \in \Gamma_{2}, \mathrm{E}_{>n} \varphi \in \Gamma_{1}$ for any $n \in \mathbb{N}$, there must exist such $\varphi \in \Gamma_{2}$ and such $n \in \mathbb{N}$ that $\mathrm{E}_{>n} \varphi \notin \Gamma_{1}$, therefore there must exist minimal (over all $\varphi \in \Gamma_{2}$ ) such $n$, call it $n_{0}$, and $\varphi_{0} \in \Gamma_{2}$. By the maximality of $\Gamma_{1}$ and the definition of $E_{=n}$ we obtain $E_{=n_{0}} \varphi_{0} \in \Gamma_{1}$. Function $\lambda$ will inform us how many copies of each MCS we should paste in the universe of created model.

Here we state and prove a useful lemma.

Lemma 5.6. Let $\Gamma_{1}, \Gamma_{2} \in \mathfrak{G}$. The following conditions are equivalent:

1. $\lambda\left(\Gamma_{1}, \Gamma_{2}\right) \neq 0$,

2. for any $\varphi \in$ FORM: if $\varphi \in \Gamma_{2}$ then $\mathrm{E}_{>0} \varphi \in \Gamma_{1}$,

3. for any $\varphi \in$ FORM: if $\mathrm{E}_{=0} \neg \varphi \in \Gamma_{1}$ then $\varphi \in \Gamma_{2}$.

Proof. $(1 \Rightarrow 2)$ Suppose there exists such $\varphi_{0} \in$ FORM that $\varphi_{0} \in \Gamma_{2}$ but $\mathrm{E}_{>0} \varphi_{0} \notin \Gamma_{1}$. By the maximality of $\Gamma_{1}$ we have that $\neg \mathrm{E}_{>0} \varphi_{0} \in \Gamma_{1}$, so by the definition of $E_{=0} E_{=0} \varphi \in \Gamma_{1}$. Thus, by the definition of $\lambda$ we obtain $\lambda\left(\Gamma_{1}, \Gamma_{2}\right)=0$.

$(2 \Rightarrow 3)$ Suppose there exists such $\varphi_{0} \in$ FORM that $\mathrm{E}_{=0} \neg \varphi_{0} \in \Gamma_{1}$ and $\varphi_{0} \notin \Gamma_{2}$. By the maximality of $\Gamma_{2} \neg \varphi_{0} \in \Gamma_{2}$ and by the consistency of $\Gamma_{1}$ it is not the case that $E_{>0} \neg \varphi_{0} \in \Gamma_{1}$.

(3 $\Rightarrow 1$ ) Suppose $\lambda\left(\Gamma_{1}, \Gamma_{2}\right)=0$. Then, by the definition of $\lambda$ there exists such $\varphi_{0} \in$ FORM that $\mathrm{E}_{=0} \varphi_{0} \in \Gamma_{1}$ and $\varphi_{0} \in \Gamma_{2}$, whence follows $\neg \varphi_{0} \notin \Gamma_{2}$.

It is easily noticeable that the function $\lambda$ will support our effort to establish a universe in the constructed model. Namely, a set $\Gamma_{2} \in \mathfrak{G}$ is a member of the universe if, and only if $\lambda\left(\Gamma_{1}, \Gamma_{2}\right) \neq 0$.

The following fact is an analog of Lemma 5.6 but it refers to ordinary modalities. 
FACT 5.7. Let $\Gamma_{1}, \Gamma_{2} \in \mathfrak{G}$. The following two conditions are equivalent:

1. for any $\varphi \in$ FORM if $\varphi \in \Gamma_{2}$ then $\diamond \varphi \in \Gamma_{1}$,

2. for any $\varphi \in$ FORM if $\square \varphi \in \Gamma_{1}$ then $\varphi \in \Gamma_{2}$.

The next fact states an important property of the consistency of a set of formulas provided that these formulas preceded by appropriate modalities build a consistent set. It will help in finding MCSs related to our set $\Gamma$ with respect to $\hat{R}$ which is defined in the following part of the section.

FACT 5.8. Let $\left\{\square \varphi_{1}, \ldots, \square \varphi_{n}, \nabla \psi\right\}$ be the consistent set of formulas. Then the set $\left\{\varphi_{1}, \ldots, \varphi_{n}, \psi\right\}$ is also consistent.

We now give a lemma which will be helpful in setting the universe of our model. Namely it assures that given a MCS $\Gamma \in \mathfrak{G}$ we will find MCSs $\Gamma^{\prime} \in \mathfrak{G}$ satisfying counting modal formulas of $\Gamma$.

LemMa 5.9. Let $\Gamma \in \mathfrak{G}$ and $\varphi \in$ FORM.

1. If $\mathrm{E}_{>0} \varphi \in \Gamma$ then there exists $\Gamma^{\prime} \in \mathfrak{G}$ such that $\varphi \in \Gamma^{\prime}$ and $\lambda\left(\Gamma, \Gamma^{\prime}\right)>0$.

2. If $\mathrm{E}_{>n} \varphi$ for all $n \in \mathbb{N}$ then there exists $\Gamma^{\prime} \in \mathfrak{G}$ such that $\varphi \in \Gamma^{\prime}$ and $\lambda\left(\Gamma, \Gamma^{\prime}\right)=\omega$.

Proof. We show that such MCSs exist by constructing them step-bystep. Let $\varphi=\varphi_{1}, \ldots, \varphi_{n}, \ldots$ be an ordered list of formulas of FORM. We define a chain of sets of formulas $\Delta_{0} \subseteq \ldots \subseteq \Delta_{n} \subset \ldots$ Let $\psi_{i}=\bigwedge\left\{\bar{\varphi} \mid \bar{\varphi} \in \Delta_{i}\right\}$ where $\bar{\varphi}_{i}=\varphi_{i}$ or $\neg \varphi_{i}$. Each $\Delta_{i}$ is such that:

i. $\mathrm{E}_{>0} \psi_{i} \in \Gamma$,

ii. $\mathbf{E}_{>n} \psi_{i} \in \Gamma$ for every $n \in \mathbb{N}$.

Gradual saturating of the set $\Delta$ resembles the one from a proof of Lindenbaum's Lemma. We proceed by induction on the degree of saturation.

BASE CASE: $n=1$. Let $\Delta_{1}=\{\varphi\}$. Then i. is the same as 1 . from the lemma.

2. Let $\Delta_{1}=\{\varphi\}$. Then ii. is the same as 2. from the lemma. 
INDUCTIVE STEP: Suppose that the conclusion holds for $n$.

1. Suppose that we have $\Delta_{n}$ such that $\mathrm{E}_{>0} \psi_{n} \in \Gamma$. By the maximality and the consistency of $\Gamma$ we have that $E_{>0}\left(\psi_{n} \wedge \varphi_{n+1}\right) \in \Gamma$ or $E_{>0}\left(\psi_{n} \wedge\right.$ $\left.\neg \varphi_{n+1}\right) \in \Gamma$ as $\mathrm{E}_{>0} \psi_{n} \in \Gamma \leftrightarrow \mathrm{E}_{>0}\left(\psi_{n} \wedge \top\right) \in \Gamma \leftrightarrow \mathrm{E}_{>0}\left(\psi_{n} \wedge\left(\varphi_{n+1} \vee\right.\right.$ $\left.\left.\neg \varphi_{n+1}\right)\right) \in \Gamma \leftrightarrow \mathrm{E}_{>0}\left(\left(\psi_{n} \wedge \varphi_{n+1}\right) \vee\left(\psi_{n} \wedge \neg \varphi_{n+1}\right)\right) \in \Gamma$. Since $\mathrm{E}_{>0}$ is the ordinary global modality $E$, it is distributive over disjunction, so we obtain $\mathrm{E}_{>0}\left(\psi_{n} \wedge \varphi_{n+1}\right) \vee \mathrm{E}_{>0}\left(\psi_{n} \wedge \neg \varphi_{n+1}\right) \in \Gamma$. So we define $\Delta_{n+1}$ as $\Delta_{n} \cup\left\{\varphi_{n+1}\right\}$ if $\mathrm{E}_{>0}\left(\psi_{n} \wedge \varphi_{n+1}\right) \in \Gamma$ or as $\Delta_{n} \cup\left\{\neg \varphi_{n+1}\right\}$ if $\mathrm{E}_{>0}\left(\psi_{n} \wedge \neg \varphi_{n+1}\right) \in \Gamma$.

2. Suppose that we have $\Delta_{n}$ such that $\mathrm{E}_{>_{n}} \psi_{n} \in \Gamma$ for each $n \in$ $\mathbb{N}$. By CT we know that $\psi_{n} \leftrightarrow\left(\psi_{n} \wedge \varphi_{n+1}\right) \vee\left(\psi_{n} \wedge \neg \varphi_{n+1}\right)$ and by Lemma 5.5 .1 we have $\mathrm{E}_{>n}\left(\psi_{n} \wedge \varphi_{n+1}\right) \in \Gamma$ for each $n \in \mathbb{N}$ or $\mathrm{E}_{>n}\left(\psi_{n} \wedge \neg \varphi_{n+1}\right) \in \Gamma$ for each $n \in \mathbb{N}$. We therefore define $\Delta_{n+1}$ as $\Delta_{n} \cup \varphi_{n+1}$ if the former is the case and as $\Delta_{n} \cup \neg \varphi_{n+1}$ otherwise.

Now we must show that $\Delta_{i}$ is consistent for every $i \in \mathbb{N}$. If it were not the case, then $\vdash \neg \psi_{i}$ and by $\mathrm{N}_{\mathrm{E}} \vdash \mathrm{E}_{=0} \psi_{i}$, so $\mathrm{E}_{=0} \psi_{i} \in \Gamma$ which contradicts the consistency of $\Gamma$. Let $\Gamma^{\prime}=\bigcup_{n \in \mathbb{N}} \Delta_{n}$. Since all $\Delta_{i}$ are consistent, $\Gamma^{\prime}$ is a MCS. We will show that $\lambda\left(\Gamma, \Gamma^{\prime}\right)>0$ in the first case and $\lambda\left(\Gamma, \Gamma^{\prime}\right)=\omega$ in the second case. Take arbitrary $\chi \in \Gamma^{\prime}$. It must have been included in a certain link of our chain of sets $-\Delta_{m}$. So if $\mathrm{E}_{>0} \psi_{m}$ it follows that $\mathrm{E}_{>0} \chi$. Since $\chi$ was arbitrary, by Lemma 5.6 we obtain $\lambda\left(\Gamma, \Gamma^{\prime}\right)>0$. Proof for the second case is analogous.

The following lemma states an interesting property of each MCS, namely the existence of a labelling formula for such a set - a formula that is included uniquely in a set labelled by it. Properties of so-defined labelling formulas strongly resemble properties of nominals from hybrid logic (for more information on the translation of hybrid logic with the global modality to modal logic with counting operators see Section 5.3 in this chapter).

LEMma 5.10. Let $\Gamma_{1}, \ldots, \Gamma_{n}$ be distinct MCSs. Then there exist labelling formulas $\varphi_{1}, \ldots, \varphi_{n} \in$ FORM such that $\varphi_{i} \in \Gamma_{j}$ for any $1 \leq i<j \leq n$ and for any $1 \leq i, j \leq n: \varphi_{i} \in \Gamma_{i}$ iff $i=j$.

Proof. We proceed by induction on $n$. 
BASE CASE: $n=2$. Let $\Gamma_{1}, \Gamma_{2}$ be two distinct MCSs. Since they are distinct, there exists a formula $\varphi$ such that $\varphi \in \Gamma_{1}$ but $\varphi \notin \Gamma_{2}$, so by maximality of $\Gamma_{2} \neg \varphi \in \Gamma_{2}$. By CT we also obtain $\vdash \neg(\varphi \wedge \neg \varphi)$.

INDUCTIVE STEP: Suppose that the conclusion holds for $n$. Let $\Gamma_{1}, \ldots, \Gamma_{n}$ be distinct MCSs and $\varphi_{1}, \ldots, \varphi_{n}$ be formulas satisfying the conditions of the lemma. We show that such a formula exists also for $\Gamma_{n+1}$ being an MCS distinct from the former ones. From the basic case we know that there exist such formulas $\psi_{1}, \ldots, \psi_{n}$ that $\psi_{i} \in \Gamma_{i}$ and $\neg \psi_{i} \in \Gamma_{n+1}$ for $i \leq n$. Let $\chi_{i}$ be a formula of the form $\varphi_{i} \wedge \psi_{i}$ for $i \leq n$ and $\bigwedge_{i \leq n} \neg \psi_{i}$ for $i=n+1$. By СТ we have that $\chi_{i} \in \Gamma_{j}$, $i, j \leq n+1$ iff $i=j$. Since $\vdash \chi_{i} \rightarrow \varphi_{i}$ for $i \leq n$, by CT we obtain $\vdash\left(\chi_{i} \wedge \chi_{j}\right) \rightarrow\left(\varphi_{i} \wedge \varphi_{j}\right), i, j \leq n$ and therefore, by taking the contrapositive $\vdash \neg\left(\chi_{i} \wedge x_{j}\right), i, j \leq n$. Since $\vdash \chi_{n+1} \rightarrow \neg \psi_{i}, i \leq n$ and $\vdash \chi_{i} \rightarrow \psi_{i}$, $i \leq n$, by CT we have $\vdash\left(x_{n+1} \wedge x_{i}\right) \rightarrow\left(\neg \psi_{i} \wedge \psi_{i}\right), i \leq n$, so by taking the contrapositive we obtain $\vdash \neg\left(X_{n+1} \wedge X_{i}\right), i \leq n$.

At the end of the preliminary part of the completeness proof of $\mathcal{M}_{K}\left(E_{n}\right)$, we introduce a notion that will be crucial in building a canonical model for $\mathcal{M}_{K}\left(E_{n}\right)$.

Definition 5.5. Let $\lambda\left(\Gamma, \Gamma^{\prime}\right)>0$. We call the satisfying family of $\Gamma$, denoted SF $(\Gamma)$, the family of MCSs together with their indices, defined as follows:

$$
\operatorname{SF}(\Gamma)=\left\{\left\{\Gamma^{\prime}\right\} \times\left\{1, \ldots, \lambda\left(\Gamma, \Gamma^{\prime}\right)\right\} \mid \Gamma^{\prime} \in \mathfrak{G}\right\} .
$$

Roughly speaking, the satisfying family of a set $\Gamma$ is a family of MCSs, each of which was copied exactly $\lambda\left(\Gamma, \Gamma^{\prime}\right)$ times. Formally speaking, each member of SF $(\Gamma)$ is an ordered pair with an MCS in the first place and an integer in the second. We identify these pairs with their first element, namely a MCS, taking integers as indices of the copies of particular MCSs. For each $\Gamma \in \mathfrak{G}$ we will call $\Gamma^{1}$ a primary copy of $\Gamma$. It is easy to note that no MCS $\Gamma^{\prime} \in \mathfrak{G}$ such that $\lambda\left(\Gamma, \Gamma^{\prime}\right)=0$ is a member of $\operatorname{SF}(\Gamma)$. It is coherent with the intuition that a set $\Gamma^{\prime}$ including a certain formula $\varphi$ such that $\mathrm{E}_{=0} \varphi \in \Gamma$ cannot be included in the same universe as $\Gamma$ because it does not satisfy all its counting formulas.

Finally, all tools necessary for building a canonical model for $\mathcal{M}_{K}\left(E_{n}\right)$ are at our disposal. 
CONSTRUCTING MODEL Now we construct a model for the arbitrarily chosen $\mathcal{M}_{K}\left(\mathrm{E}_{n}\right)-\mathrm{MCS}$.

Definition 5.6. We have the logic $\mathcal{M}_{K}\left(E_{n}\right)$ and the $\mathcal{M}_{K}\left(E_{n}\right)-M C S \Gamma$. We construct a model $\hat{\mathfrak{M}}=\langle\hat{W}, \hat{R}, \hat{V}\rangle$ for it in the following way

1. $\hat{W}=\operatorname{SF}(\Gamma)$, where $\Gamma$ is arbitrarily chosen $\mathcal{M}_{K}\left(\mathrm{E}_{n}\right)$-maximal consistent extension for $\Gamma^{-}$,

2. $\hat{R}=\left\{\left\langle\Gamma^{\prime}, \Gamma^{\prime \prime}\right\rangle \mid \Gamma^{\prime}, \Gamma^{\prime \prime} \in \hat{W}\right.$ and if $\varphi \in \Gamma^{\prime \prime}$ then $\nabla \varphi \in \Gamma^{\prime}$ for all $\left.\varphi \in \Gamma^{\prime \prime}\right\}$

3. $\hat{V}:$ PROP $\longrightarrow \mathcal{P}(\hat{W}): \hat{V}(p)=\left\{\Gamma^{\prime} \in \hat{W} \mid p \in \Gamma^{\prime}\right\}$.

Now, before we state and prove the Existence Lemma and the Truth Lemma, we need to show that the universe of the model, namely SF $(\Gamma)$ is not only the satisfaction family for $\Gamma$ itself, but also for each member of the universe. In order to prove this, it suffices to show that the satisfaction relation for $\Gamma$ defined as $\{\{\Gamma\} \times \operatorname{SF}(\Gamma) \mid \Gamma \in \hat{W}\}^{4}$ is the equivalence relation and, therefore, that $S F(\Gamma)$ is a cluster with respect to this relation.

LeMma 5.11. $\left\{\left\{\Gamma^{\prime}\right\} \times S F\left(\Gamma^{\prime}\right) \mid \Gamma^{\prime} \in \mathfrak{G}\right\}$ is an equivalence relation.

Proof. We prove that $\left\{\left\{\Gamma^{\prime}\right\} \times S F\left(\Gamma^{\prime}\right) \mid \Gamma^{\prime} \in \mathfrak{G}\right\}$ is reflexive, transitive and symmetric.

Reflexivity. We show that for each $\Gamma^{\prime} \in \mathfrak{G}, \Gamma^{\prime} \in S F\left(\Gamma^{\prime}\right)$. It suffices to prove that $\lambda\left(\Gamma^{\prime}, \Gamma^{\prime}\right)>0, \Gamma^{\prime} \in \mathfrak{G}$. By $\operatorname{lnt}_{E_{n}}$ we have $\mathrm{E}_{>0} \varphi \in \Gamma^{\prime}$ for each $\varphi \in \Gamma^{\prime}$. By Lemma 5.6 we obtain $\lambda\left(\Gamma^{\prime}, \Gamma^{\prime}\right)>0$.

Transitivity. Let $\Gamma^{\prime}, \Gamma^{\prime \prime}, \Gamma^{\prime \prime \prime} \in \mathfrak{G}$. We show that if $\Gamma^{\prime \prime} \in S F\left(\Gamma^{\prime}\right)$ and $\Gamma^{\prime \prime \prime} \in S F\left(\Gamma^{\prime \prime}\right)$ then $\Gamma^{\prime \prime \prime} \in S F\left(\Gamma^{\prime}\right)$. Suppose that $\lambda\left(\Gamma^{\prime}, \Gamma^{\prime \prime}\right)>0$ and $\lambda\left(\Gamma^{\prime \prime}, \Gamma^{\prime \prime \prime}\right)>0$. By Lemma 5.6 we have $\mathrm{E}_{>0} \varphi \in \Gamma^{\prime}$ for each $\varphi \in \Gamma^{\prime \prime}$ and $\mathrm{E}_{>0} \psi \in \Gamma^{\prime \prime}$ for each $\psi \in \Gamma^{\prime \prime \prime}$. Assume that $\lambda\left(\Gamma^{\prime}, \Gamma^{\prime \prime \prime}\right)=0$. Then there is such $\psi_{0} \in \Gamma^{\prime \prime \prime}$ that $\mathrm{E}_{=0} \psi_{0} \in \Gamma^{\prime}$. By $\operatorname{lnt}_{\mathrm{E}_{n}}$ we obtain $\mathrm{E}_{>0}\left(\mathrm{E}_{=0} \psi_{0}\right) \in \Gamma^{\prime}$. By Tot $1_{E_{n}}$ we have $E_{=0} \neg\left(E_{>0}\left(E_{=0} \psi_{0}\right)\right) \in \Gamma^{\prime}$. Since $\lambda\left(\Gamma^{\prime}, \Gamma^{\prime \prime}\right)>0$, we have $\mathrm{E}_{>0}\left(\mathrm{E}_{=0} \psi_{0}\right) \in \Gamma^{\prime \prime}$. Since $\lambda\left(\Gamma^{\prime \prime}, \Gamma^{\prime \prime \prime}\right)>0, \mathrm{E}_{>0} \psi_{0} \in \Gamma^{\prime \prime}$. By Tot $1_{\mathrm{E}_{n}}$ we obtain $\mathrm{E}_{=0}\left(\mathrm{E}_{=0} \psi_{0}\right) \in \Gamma^{\prime \prime}$. Contradiction.

$4 \overline{\text { We identify a pair belonging to } S F}(\Gamma)$ with its first element. 
Symmetry. Let $\Gamma^{\prime}, \Gamma^{\prime \prime} \in \mathfrak{G}$. We show that if $\Gamma^{\prime \prime} \in \operatorname{SF}\left(\Gamma^{\prime}\right)$ then $\Gamma^{\prime} \in \operatorname{SF}\left(\Gamma^{\prime \prime}\right)$, so $\lambda\left(\Gamma^{\prime \prime}, \Gamma^{\prime}\right)>0$ whenever $\lambda\left(\Gamma^{\prime}, \Gamma^{\prime \prime}\right)>0$. Assume that $\lambda\left(\Gamma^{\prime}, \Gamma^{\prime \prime}\right)>0$ and $\lambda\left(\Gamma^{\prime \prime}, \Gamma^{\prime}\right)=0$. Hence we have such $\varphi_{0} \in \Gamma^{\prime}$ that $\mathrm{E}_{=0} \varphi_{0} \in \Gamma^{\prime \prime}$. Since $\lambda\left(\Gamma^{\prime}, \Gamma^{\prime \prime}\right)>0$ we have $\mathrm{E}_{>0}\left(\mathrm{E}_{=0} \varphi_{0}\right) \in \Gamma^{\prime}$. By $\operatorname{lnt}_{\mathrm{E}_{n}}$ we obtain $\mathrm{E}_{>0} \varphi_{0} \in \Gamma^{\prime}$ and by $\operatorname{Tot}_{\mathrm{E}_{n}}$ we have $\mathrm{E}_{=0}\left(\mathrm{E}_{=0} \varphi_{0}\right) \in \Gamma^{\prime}$. Contradiction.

Since we proved that $\left\{\left\{\Gamma^{\prime}\right\} \times S F\left(\Gamma^{\prime}\right) \mid \Gamma^{\prime} \in \mathfrak{G}\right\}$ is the equivalence relation, it becomes obvious that $\hat{W}$ is the equivalence class of $\left\{\left\{\Gamma^{\prime}\right\} \times \operatorname{SF}\left(\Gamma^{\prime}\right) \mid \Gamma^{\prime} \in \mathfrak{G}\right\}$ and hence that $\hat{W}=\operatorname{SF}(\Gamma)=\operatorname{SF}\left(\Gamma^{\prime}\right)$ for all $\Gamma^{\prime} \in \hat{W}$.

Now we have all that we need to state and prove two lemmas of crucial importance for the whole argument.

Lemma 5.12 (Existence Lemma). Let $\hat{\mathfrak{M}}=\langle\hat{W}, \hat{R}, \hat{V}\rangle$ be a model for $\mathcal{M}_{\mathrm{K}}\left(\mathrm{E}_{n}\right)$ and $\Gamma_{0} \in \hat{W}$. Then the following conditions hold

1. $\mathrm{E}_{>n} \varphi \in \Gamma_{0}$ iff $\operatorname{Card}\left(\left\{\Gamma^{\prime} \in \hat{W} \mid \varphi \in \Gamma^{\prime}\right\}\right)>n$.

2. If $\diamond \varphi \in \Gamma_{0}$ then there exists such $\Gamma^{\prime} \in \hat{W}$ that $\Gamma_{0} \hat{R} \Gamma^{\prime}$ and $\varphi \in \Gamma^{\prime}$.

Proof. Let $\Gamma_{0} \in \hat{W}$.

1. $(\Leftarrow)$ Assume that $\operatorname{Card}\left(\left\{\Gamma^{\prime} \in \hat{W} \mid \varphi \in \Gamma^{\prime}\right\}\right)>n$. We show that $\mathrm{E}_{>n} \varphi \in \Gamma_{0}$. If there exists $\Gamma^{\prime} \in \hat{W}$ such that $\varphi \in \Gamma^{\prime}$ and $\lambda\left(\Gamma_{0}, \Gamma^{\prime}\right)>n$ then the case is trivial. Indeed, since $\lambda\left(\Gamma_{0}, \Gamma^{\prime}\right)=m$ iff $\mathrm{E}_{>m-1} \varphi \in \Gamma_{0}$ for all $\varphi \in \Gamma^{\prime}$ and since $m-1 \geq n$, by Num we obtain $\mathrm{E}_{>n} \varphi \in \Gamma_{0}$. Suppose then that for all $\Gamma^{\prime} \in \hat{W}$ such that $\varphi \in \Gamma^{\prime}, \lambda\left(\Gamma_{0}, \Gamma^{\prime}\right) \leq n$. We name the family $\bar{\Gamma}_{\varphi}=\left\{\Gamma^{\prime} \in \hat{W} \mid \varphi \in \Gamma^{\prime}\right\}$. Without loss of generality, we assume that $\bar{\Gamma}_{\varphi}$ is finite and therefore, includes finite subfamily of distinct primary copies, name them $\Gamma_{1}, \ldots, \Gamma_{m}$. The rest of the members of $\bar{\Gamma}_{\varphi}$ are copies of one of $\Gamma_{1}, \ldots, \Gamma_{m}$ (see Fig. 2). Let $\operatorname{Card}\left(\bar{\Gamma}_{\varphi}\right)=k$. We have that $\lambda\left(\Gamma_{0}, \Gamma_{i}\right)=n_{i}$ for $i \leq m$. By Definition 5.5 it follows that we have $n_{i}$ copies of each $\Gamma_{i}, i \leq m$. Hence, we obtain $\sum_{i=1}^{m} n_{i}=k$ and $k>n$. Now, by Definition 5.4 each $n_{i}$ is determined by such formula $\psi_{i} \in \Gamma_{i}$ that $\mathrm{E}_{=n_{i}} \psi_{i} \in \Gamma_{0}$ and $n_{i}=\min \left\{n \mid \mathrm{E}_{=n} \psi \in\right.$ $\Gamma_{0}$ and $\left.\psi \in \Gamma_{i}\right\}$. Let $\psi_{1} \in \Gamma_{1}, \ldots, \psi_{m} \in \Gamma_{m}$ be such formulas that $\mathrm{E}_{=n_{i}} \psi_{i} \in \Gamma_{0}, i \leq m$. Lemma 5.10 guarantees the existence of the socalled labelling formula for each $\Gamma_{i}, i \leq m$. Let $\chi_{1} \in \Gamma_{1}, \ldots, \chi_{m} \in \Gamma_{m}$ 
be such labelling formulas. Note that they distinguish primary copies from each other but do not distinguish copies of certain primary copy. Let $\vartheta_{i}=\varphi \wedge \psi_{i} \wedge x_{i}, i \leq m$. It is easy to notice that by maximality of $\Gamma_{i}, i \leq m, \vartheta_{i} \in \Gamma_{i}, i \leq m$. $\vartheta_{i}, i \leq m$ also work as labelling formulas, so we have $\vdash \neg\left(\vartheta_{i} \wedge \vartheta_{j}\right)$ for $i, j \leq m, i \neq j$. Indeed, since $\vdash \vartheta_{i} \rightarrow \psi_{i}$, and therefore $\vdash\left(\vartheta_{i} \wedge \vartheta_{j}\right) \rightarrow\left(\psi_{i} \wedge \psi_{j}\right)$, we have $\vdash \neg\left(\vartheta_{i} \wedge \vartheta_{j}\right)$ for $i \neq j$. The last property of $\vartheta_{i}, i \leq m$ that we are going to use is that $\mathrm{E}_{=n_{i}} \vartheta_{i} \in \Gamma_{0}$. In fact, since $\vdash \vartheta_{i} \rightarrow \chi_{i}$ and $\mathrm{E}_{=n_{i}} \chi_{i} \in \Gamma_{0}$ by Lemma 5.4 we obtain $\mathrm{E}_{=l} \vartheta_{i} \in \Gamma_{0}, l \leq n_{i}$. By Definition 5.4 we have that for all formulas $\varphi \in \Gamma_{i}, i \leq m, \mathrm{E}_{>n_{i}-1} \varphi \in \Gamma_{0}$. By the definition of $\mathrm{E}_{=n}$ we get $\mathrm{E}_{=n_{i}} \vartheta_{i} \in \Gamma_{0}, i \leq m$. Let $\mu=\bigvee_{i \leq m} \vartheta_{i}$. Obviously, $\mu \in \Gamma_{0}$. Since $\vartheta_{1}, \ldots, \vartheta_{m} \in \Gamma_{0}$ and $\mu \in \Gamma_{0}$ fulfil the conditions of Lemma 5.3, it is the case that $\mathrm{E}_{=\sum_{i=1}^{m} n_{i}} \mu=\mathrm{E}_{=k} \mu \in \Gamma_{0}$. Since $\vdash \mu \leftrightarrow \varphi \wedge \bigvee_{i \leq m}\left(\psi_{i} \wedge \chi_{i}\right)$, we obtain $\vdash \mu \rightarrow \varphi$ and hence by $\mathrm{N}_{\mathrm{E}} \vdash \mathrm{E}_{=0} \neg(\mu \rightarrow \varphi)$. Thus, by $\mathrm{K}_{\mathrm{E}_{n}}$ $\vdash \mathrm{E}_{>k-1} \mu \rightarrow \mathrm{E}_{>k-1} \varphi$. Again, by the definition of $\mathrm{E}_{=n}$ and by the fact that $\mathrm{E}_{=k} \mu \in \Gamma_{0}$ we have $\mathrm{E}_{>k-1} \mu \in \Gamma_{0}$, so by $\mathrm{MP} \mathrm{E}_{>k-1} \varphi \in \Gamma_{0}$. Since $k-1 \geq n$, by Num we obtain $\mathrm{E}_{>n} \varphi \in \Gamma_{0}$.

$(\Rightarrow)$ Assume that $\mathrm{E}_{>n} \varphi \in \Gamma_{0}$. What we have to show is that $\operatorname{Card}\left(\left\{\Gamma^{\prime} \in \hat{W} \mid \varphi \in \Gamma^{\prime}\right\}\right)>n$. Since $\mathrm{E}_{>n} \varphi \in \Gamma_{0}$, by Lemma 5.6 we obtain that there exists such $\Gamma^{\prime} \in \hat{W}$ that $\varphi \in \Gamma^{\prime}$ and $\lambda\left(\Gamma_{0}, \Gamma^{\prime}\right)>0$. Without loss of generality, suppose that the family of such $\Gamma^{\prime}$, name it $\bar{\Gamma}_{\varphi}$ is finite. We distinguish subfamily $\Gamma_{1}, \ldots, \Gamma_{m}$ of $\bar{\Gamma}_{\varphi}$ consisting of primary copies only, the rest of the sets being copies of primary copies. Finiteness of $\bar{\Gamma}_{\varphi}$ entails $\lambda\left(\Gamma_{0}, \Gamma_{i}\right)<\omega, i \leq m$. We prove that $k>n$, where $k=\sum_{i=1}^{m} n_{i}$. Analogously to the first part of the proof, we define formulas $\vartheta_{i}=\varphi \wedge \psi_{i} \wedge \chi_{i}$ for $i \leq$ $m$. Let $\mu=\bigvee_{i \leq m} \vartheta_{i}$. We have $\vdash \mu \leftrightarrow \varphi \wedge \bigvee_{i \leq m}\left(\psi_{i} \wedge \vartheta_{i}\right)$. Let $v=\bigvee_{i \leq m}\left(\psi_{i} \wedge \vartheta_{i}\right)$. We have then that $\mu \leftrightarrow \varphi \wedge v$. Therefore, by $\mathrm{CT}$ we have that $\vdash(\mu \vee(\varphi \wedge \neg v)) \leftrightarrow \varphi$. Additionally, by CT, for all $i \leq m$ we have that $\vdash \neg\left(\vartheta_{i} \wedge(\varphi \wedge \neg v)\right)$. Finally, we state that $\mathrm{E}_{=0}(\varphi \wedge \neg v) \in \Gamma_{0}$. Indeed, if it were not the case we would have $\mathrm{E}_{>0}(\varphi \wedge \neg v) \in \Gamma_{0}$. Hence, by Lemma 5.6 there would exist such $\Gamma^{\prime} \in \hat{W}$ that $\varphi \wedge \neg v \in \Gamma^{\prime}$ and $\lambda\left(\Gamma_{0}, \Gamma^{\prime}\right)>0$. It would be the case that $\Gamma^{\prime} \in \bar{\Gamma}_{\varphi}$, name it $\Gamma_{h}, h \leq m$ (without loss of generality, we can take it as one of the primary copies). So we would have $\vartheta_{h} \in \Gamma_{h}$. Together with $\vdash \neg\left(\vartheta_{h} \wedge(\varphi \wedge \neg v)\right)$ it would contradict 


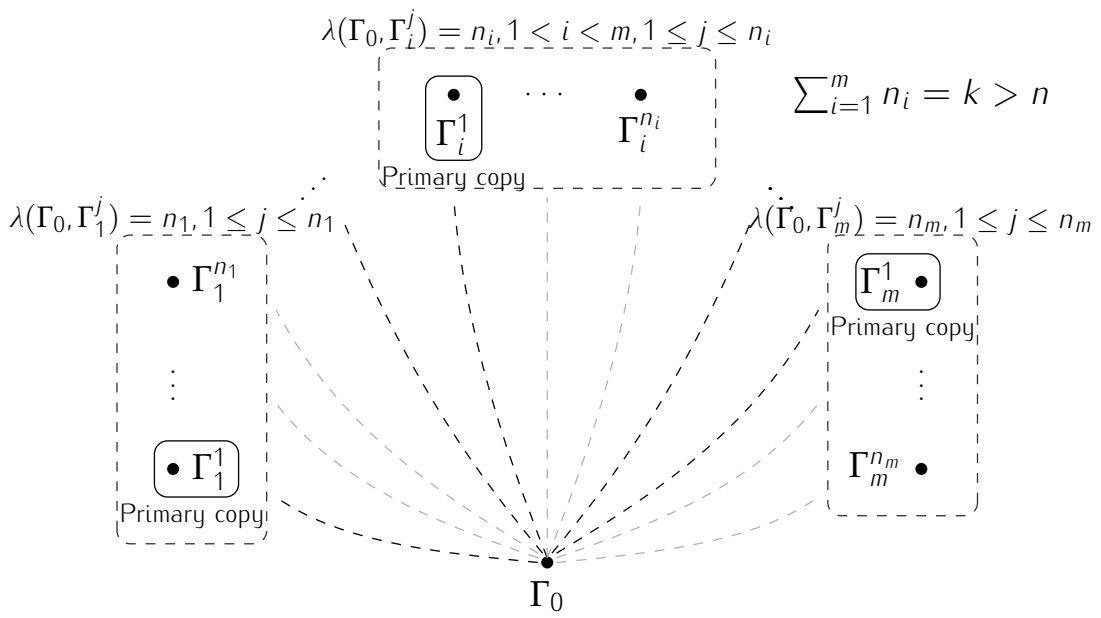

Figure 2: The family $\bar{\Gamma}_{\varphi}$.

the consistency of $\Gamma_{h}$. Let $\xi=\mu \vee(\varphi \wedge \neg v)$. Since $\vdash \neg\left(\vartheta_{i} \wedge \vartheta_{j}\right)$ for $i, j \leq m, i \neq j, \vdash \neg\left(\vartheta_{i} \wedge(\varphi \wedge \neg v)\right)$ for $i \leq m, \mathrm{E}_{=n_{i}} \vartheta \in \Gamma_{0}$ for $i \leq m$ and $E_{=0}(\varphi \wedge \neg v) \in \Gamma_{0}$, this set of formulas satisfies the conditions of Lemma 5.3, so we have $E_{=k} \xi \in \Gamma_{0}$. Since $\vdash \xi \leftrightarrow \varphi$ and the fact that $\mathrm{E}_{=n}$ is monotone over equivalence, we have $\mathrm{E}_{=k} \varphi \in \Gamma_{0}$. Hence $k>n$ for if it were not the case, by definition of $E_{=n}$ we would have $\neg \mathrm{E}_{>l \varphi} \in \Gamma_{0}$ for some $l \leq n$ and at the same time, by Num $\mathrm{E}_{>l} \varphi \in \Gamma_{0}$ which would contradict the consistency of $\Gamma_{0}$.

2. Assume that $\nabla \varphi \in \Gamma_{0}$. We show that there exists such $\Gamma^{\prime} \in \hat{W}$ that $\Gamma_{0} \hat{R} \Gamma^{\prime}$ and $\varphi \in \Gamma^{\prime}$. Let $\Sigma=\left\{\psi \mid \square \psi \in \Gamma_{0}\right\}$. By Fact 5.8 the set $\Sigma \cup\{\varphi\}$ is consistent and by Lindenbaum's lemma it can be extended to a MCS. We name this extension $\Gamma^{\prime}$. Now we must show that $\Gamma_{0} \hat{R} \Gamma^{\prime}$. Since $\Sigma \subseteq \Gamma^{\prime}$, Fact 5.7 and the definition of $R$ assure that $\Gamma_{0} \hat{R} \Gamma^{\prime}$. The only thing that is left to show is that $\Gamma^{\prime} \in \hat{W}$. It suffices to prove that $\Gamma^{\prime} \in S F\left(\Gamma_{0}\right)$, so that $\lambda\left(\Gamma_{0}, \Gamma^{\prime}\right)>0$. Since $\Gamma_{0} \hat{R} \Gamma^{\prime}$ it is the case that $\nabla \psi \in \Gamma_{0}$ for all $\psi \in \Gamma^{\prime}$. By axiom 3 we have that $\mathrm{E}_{>0} \psi \in \Gamma_{0}$ for all $\diamond \psi \in \Gamma_{0}$. Therefore, for all $\psi \in \Gamma^{\prime}$ we have $\mathrm{E}_{>0} \psi \in \Gamma_{0}$, which drives to the conclusion.

Since the first part of the Existence Lemma was of the form of equivalence, we therefore assured satisfiability of all formulas of one of the 
forms: $\mathrm{E}_{<n} \varphi, \mathrm{E}_{=n} \varphi$ for $\varphi \in$ FORM. Proof of the first part of the above lemma is much more complicated than the second and it is of great importance to grasp the idea standing behind it. The sole definition of the satisfaction family of a set $\Gamma$ (Def. 5.5) does not assure that the condition 1. of the Existence Lemma holds. Thus, a reader might state such a problem: Let $\Gamma_{1}, \Gamma_{2} \in \mathfrak{G}$ and $\lambda\left(\Gamma_{1}, \Gamma_{2}\right)=n$. It means that we copy $\Gamma_{2} n$ times. But, the reader might ask, what guarantee do we have that there is no such $\Gamma_{3} \in \mathfrak{G}$ that $\lambda\left(\Gamma_{1}, \Gamma_{3}\right)=m>0$ and $\varphi \in \Gamma_{3}$ ? It would mean that there are at least $n+m$ such sets $\Gamma^{\prime}$ in $\operatorname{SF}\left(\Gamma_{1}\right)$ that $\varphi \in \Gamma^{\prime}$ and that would affect satisfaction of the formula $\mathrm{E}_{=n} \varphi \in \Gamma^{\prime}$. Now, the proof of the latter part of the Existence Lemma states that it cannot be the case. For if it were, it would also be the case that $\mathrm{E}_{>n+m-1} \varphi \in \Gamma_{1}$ (we showed this using labelling formulas) and that would contradict the consistency of $\Gamma_{1}$.

Now we can prove a lemma that is an easy consequence of the former definitions and results.

Lemma 5.13 (Truth Lemma). Let $\hat{\mathfrak{M}}=\langle\hat{W}, \hat{R}, \hat{V}\rangle$ be a model constructed for $\mathcal{M}_{K}\left(E_{n}\right)$-MCS $\Gamma$. We have $\hat{\mathfrak{M}}, \Gamma \models \varphi$ iff $\varphi \in \Gamma$.

Proof. We proceed by induction on the degree of $\varphi$. Basic case holds due to the definition of $\hat{V}$, Boolean cases follow from maximality of $\Gamma$.

$\varphi=\diamond \psi .(\Rightarrow)$ Assume that $\hat{\mathfrak{M}}, \Gamma \models \diamond \psi$. By Definition 5.2 there exists $\Gamma^{\prime} \in \hat{W}$ such that $\Gamma \hat{R} \Gamma^{\prime}$ and $\hat{\mathfrak{M}}, \Gamma^{\prime} \models \psi$. By the inductive hypothesis $\psi \in \Gamma^{\prime}$. Hence, by definition of $\hat{R}, \nabla \psi \in \Gamma$.

$(\Leftarrow)$ Assume that $\nabla \psi \in \Gamma$. Then, by the Existence Lemma, there exists $\Gamma^{\prime} \in \hat{W}$ such that $\Gamma \hat{R} \Gamma^{\prime}$ and $\psi \in \Gamma^{\prime}$. By the inductive hypothesis $\hat{\mathfrak{M}}, \Gamma^{\prime}=\psi$ and since $\Gamma \hat{R} \Gamma^{\prime}, \hat{\mathfrak{M}}, \Gamma \models \diamond \psi$.

$\varphi=\mathrm{E}_{>n} \psi .(\Rightarrow)$ Assume that $\hat{\mathfrak{M}}, \Gamma \models \mathrm{E}_{>n} \psi$. By Definition 5.2 $\operatorname{Card}(\{\Gamma \in\{\hat{W} \mid \mathfrak{M}, \Gamma \Vdash \psi\})>n$. By induction hypothesis $\operatorname{Card}(\{\Gamma \in$ $\hat{W} \mid \psi \in \Gamma\})>n$. By the Existence Lemma we obtain $\mathrm{E}_{>n} \psi \in \Gamma$.

$(\Leftarrow)$ Assume that $\mathrm{E}_{>_{n}} \psi \in \Gamma$. By the Existence Lemma we have $\operatorname{Card}(\{\Gamma \in \hat{W} \mid \psi \in \Gamma\})>n$ and by the inductive hypothesis $\operatorname{Card}(\{\Gamma \in \hat{W} \mid \mathfrak{M}, \Gamma \Vdash \psi\})>n$. Hence, by Definition 5.2, $\hat{\mathfrak{M}}, \Gamma \Vdash \mathrm{E}_{>n} \psi$. 
We can now conclude our considerations with two theorems.

Theorem 5.14 (Strong Completeness of $\mathcal{M}_{K}\left(E_{n}\right)$ ). Logic $\mathcal{M}_{K}\left(E_{n}\right)$ is strongly complete with respect to the class of all frames $\mathfrak{F}=\langle W, R\rangle$.

Proof. By Lemma 5.2 it suffices to show that every $\mathcal{M}_{K}\left(E_{n}\right)$-consistent set has a model based on a frame of the class of all frames. Given the $\mathcal{M}_{K}\left(E_{n}\right)$-consistent set $\Gamma^{-}$, we can extend it to a MCS $\Gamma$ and build a canonical model for it in a way showed above. As we proved, a $\mathcal{M}_{K}\left(\mathrm{E}_{n}\right)$-model $\hat{\mathfrak{M}}=\langle\hat{W}, \hat{R}, \hat{V}\rangle$ is based on a frame of the class of all frames. Since $\Gamma^{-} \subseteq \Gamma$, by the Truth Lemma we obtain $\hat{\mathfrak{M}}, \Gamma \Vdash \Gamma^{-}$. $\dashv$

THEOREM 5.15 (Adequacy of $\mathcal{M}_{K}\left(\mathrm{E}_{n}\right)$ ). Logic $\mathcal{M}_{\mathrm{K}}\left(\mathrm{E}_{n}\right)$ is strongly adequate with respect to the class of all frames $\mathfrak{F}=\langle W, R\rangle$.

Proof. Soundness follows from Theorem 5.1. Strong completeness follows from Theorem 5.14.

The reader has probably already noticed that although the proof of adequacy was conducted for the logic $\mathcal{M}_{K}\left(E_{n}\right)$, it can be easily generalised on the case of all normal modal logics adequate with respect to a certain class of frames. It is so due Axiom 3 that makes the second part of the Existence Lemma always hold.

\subsection{COMPLEXITY}

In this section we prove several theorems establishing the computational complexity of modal logic with global counting operators defined over various classes of frames. We start with the result on the complexity of the logic $\mathcal{M}_{K}\left(E_{n}\right)$. In the remainder of the section we proceed to the cases of other frame classes.

TheOREM 5.16 (Finite Model Property). The logic $\mathcal{M}_{K}\left(E_{n}\right)$ has the effective finite model property with the bounding function $\mu=$ $2^{\operatorname{Card}(\{\operatorname{Sub}(\varphi)\})+\log (n+1)}$ for any given input formula $\varphi$, where $\operatorname{Sub}(\varphi)$ is the set of all subformulas of $\varphi$ and $n=\max \left\{m: E_{>m} \psi \in \operatorname{Sub}(\varphi)\right\}$, where $n$ is coded in binary, i.e., whenever $\varphi$ has a model, it also has a model of the size not exceeding $2^{\operatorname{Card}(\{\operatorname{Sub}(\varphi)\})+\log (n+1)}$. 
Proof. Let $\varphi$ be a formula satisfiable on a (possibly infinite) model $\mathfrak{M}=\langle W, R, V\rangle$. We show that there exists a finite model $\mathfrak{M}^{\prime}=$ $\left\langle W^{\prime}, R^{\prime}, V^{\prime}\right\rangle$ on which $\varphi$ is satisfiable.

We proceed in two steps.

In the first step we exploit a filtration-like method to divide the universe $W$ into a finite number of equivalence classes. We fix the equivalence relation ${ }^{m} \operatorname{Sub}(\varphi)$ in the following way:

$$
w \leadsto \operatorname{Sub}(\varphi) v \text { iff for all } \psi \in \operatorname{Sub}(\varphi)(\mathfrak{M}, w \Vdash \psi \text { iff } \mathfrak{M}, v \Vdash \psi) .
$$

It is straightforward that there are only finitely many such equivalence classes, namely $2^{\operatorname{Card}(\operatorname{Sub}(\varphi))}$.

In the second step, we abandon the ordinary filtration procedure. Instead of merging all worlds from the equivalence classes, we reduce the cardinality of each class in the following manner. Let $[w] \subseteq W$ be an arbitrary $\leadsto \operatorname{Sub}(\varphi)$-equivalence class. If $\operatorname{Card}([w])>n+1$ then we delete all but $n+1$ arbitrary worlds from $[w]$. If $\operatorname{Card}([w]) \leq n+1$ then we leave $[w]$ unchanged. Next, from each reduced equivalence class $[w]^{\prime}$ we pick an arbitrary representative $w_{0}$. We set a new model $\mathfrak{M}^{\prime}=\left\langle W^{\prime}, R^{\prime}, V^{\prime}\right\rangle$ where $\left.W^{\prime}=\bigcup_{[w] \in W / W^{\prime} \operatorname{Sub}(\varphi)}\left[W^{\prime}\right], R^{\prime}=R\right\rfloor W^{\prime} \cup$ $\bigcup_{[w],[v] \in W / W^{\prime} \operatorname{sub}(\varphi)}\left\{\left\langle w, v_{0}\right\rangle \mid w \in[w]^{\prime},\langle w, v\rangle \in R, v \in[v] \backslash[v]^{\prime}, v_{0} \in[v]^{\prime}\right\}$ and $\left.V^{\prime}=V\right\rfloor W^{\prime}$. Of course, $w$ such that $\mathfrak{M}, w \Vdash \varphi$ remains in $W^{\prime}$.

We prove that $\mathfrak{M}^{\prime}$ is a model for $\varphi$ by induction on the complexity of the elements of $\operatorname{Sub}(\varphi)$.

The Boolean cases are obvious and follow directly from the definition of $W^{\prime}$ and $V^{\prime}$.

Suppose that a formula $\nabla \psi$ is satisfiable on $\mathfrak{M}$. It means that there exists such $w \in W$ that $\mathfrak{M}, w \Vdash \Delta \psi$. We pick an arbitrary $w^{\prime} \in[w]^{\prime}$. By definition of ${ }^{m} \operatorname{Sub}(\varphi)$ it follows that $\mathfrak{M}, w^{\prime} \Vdash \Delta \psi$. Consequently, we can find $v \in W$ such that $\left(w^{\prime}, v\right) \in R$ and $\mathfrak{M}, v \Vdash \psi$. If $v \in[v]^{\prime}$ then we have also $\mathfrak{M}^{\prime}, w^{\prime} \Vdash \diamond \psi$. Otherwise, by definition of $\aleph^{\rightsquigarrow} \operatorname{Sub}(\varphi)$ and $R^{\prime}$ there exists $v_{0} \in[v]^{\prime}$ such that $\left(w^{\prime}, v_{0}\right) \in R^{\prime}$ and $\mathfrak{M}$, $v_{0} \Vdash \psi$. Therefore, $\diamond \psi$ is satisfiable on $\mathfrak{M}$.

Now, assume that a formula $\mathrm{E}_{>m} \psi$ is satisfied by $\mathfrak{M}$. It means that there exist more than $m$ worlds in which $\psi$ holds. Two cases may occur. Either $\psi$ holds in elements of (at least one) equivalence class $[w]$ such that $\operatorname{Card}([w])>n$. Then, by the construction of $[w]^{\prime}$, we 
obtain that $E_{>m}$ is satisfied by $\mathfrak{M}^{\prime}$. Otherwise $\psi$ holds in elements of equivalence classes $[w]_{i_{1}}, \ldots,[w]_{i_{k}}$ such that $\operatorname{Card}\left([w]_{i_{j}}\right) \leq n$ and $\sum_{j=1}^{k} \operatorname{Card}\left([w]_{i_{j}}\right)>n$. But by construction of $[w]_{i_{j}}^{\prime}$ these classes remained unchanged in $W^{\prime}$, therefore $\sum_{j=1}^{k} \operatorname{Card}\left([w]_{i_{j}}^{\prime}\right)>n$. It follows that $E_{>m}$ is satisfied by $\mathfrak{M}^{\prime}$. The reduction of the size of $W$ cannot disturb satisfiability of the formulas $E_{<m} \psi$ on $\mathfrak{M}^{\prime}$.

Since $\operatorname{Card}(W / \lll \operatorname{Sub}(\varphi)) \leq 2^{\operatorname{Sub}(\varphi)}$ and for each $[W]^{\prime}$ obtained from $[W] \in W /{ }_{\operatorname{Sub}(\varphi)} \operatorname{Card}\left([w]^{\prime}\right) \leq n+1$, it is clear that $\operatorname{Card}\left(W^{\prime}\right) \leq 2^{\operatorname{Card}(\{\operatorname{Sub}(\varphi)\})+\log (n+1)}$. This completes the proof.

THEOREM 5.17. The logic $\mathcal{M}_{K}\left(\mathrm{E}_{n}\right)$ is NEXPTIME-complete.

Proof. Membership of $\mathcal{M}_{K}\left(E_{n}\right)$ in NExPTIME is a corollary to Theorem 5.16. It also can be proven by giving a standard translation function from $\mathcal{M}_{K}\left(E_{n}\right)$ to $\mathcal{C}^{2}$ (two-variable fragment of first order logic with counting quantifiers) where number restrictions are given in binary, which was proven to be NExPTIME-complete [79]. However, in the light of our considerations, a precise bound of the size of a model is desirable, since we might want to turn the indeterministic procedure into a deterministic one ${ }^{5}$. Theorem 5.16 delivers such a bound.

NEXPTIME-hardness is proven by reducing a standard exponential bounded tiling problem [99].

By a tile type $T$ we understand a quadruple of colours $\left\langle\operatorname{left}_{T}, \operatorname{up}_{T}\right.$, right $_{T}$, down $\left._{T}\right\rangle$. Given a finite set of tile-types $\mathrm{T}=$ $\left\{T_{0}, \ldots, T_{m}\right\}$ and a finite square grid $k \times k$, the bounded tiling problem is: Does there exist a function $\tau: k \times k \longrightarrow \mathrm{T}$ (a tiling) such that the following all hold?

(a) $\tau(0,0)=T_{0}$

(b) $\operatorname{up}_{\tau(i, j)}=\operatorname{down}_{\tau(i, j+1)}, 1 \leq i \leq k, 1 \leq j<k$,

(c) $\operatorname{right}_{\tau(i, j)}=\operatorname{left}_{\tau(i+1, j)}, 1 \leq i<k, 1 \leq j \leq k$.

The bounded tiling problem was proven to be NP-complete ([99]), i.e., it is non-deterministically solvable in polynomial time in the size of a grid and the number of tiles.

5 In fact, we do so by providing the (op) condition in the following part of the chapter. 
In the following lemma we show that given a finite set of tile types $\mathrm{T}=\left\{T_{0}, \ldots, T_{m}\right\}$ and $k \in \mathbb{N}$ (coded in binary), there exists a $\mathcal{M}_{\mathrm{K}}\left(\mathrm{E}_{n}\right)$ formula $\phi$ computable in polynomial time in $m$ and $\log (k)$ such that the following equivalence holds:

$$
\begin{aligned}
& \text { A tiling } \tau \text { for } \mathrm{T} \text { and } k \times k \text { exists } \\
& \text { iff } \phi \text { is satisfiable. }
\end{aligned}
$$

Since the size of $\phi$, for which (1) holds is logarithmic in the size of a grid, the satisfiability problem for $\phi$ is NExPTIME-complete (cf. $[7,61])$.

LEMMA 5.18. The exponential bounded tiling problem is reducible in polynomial time to the satisfiability problem for the logic $\mathcal{M}_{K}\left(E_{n}\right)$.

Proof. To construct $\phi$ we proceed in several steps. First, we fix a set $\mathcal{T}=\left\{t_{0}, \ldots, t_{m}\right\}$ of propositional variables that will stand for tile types. We also introduce two adjacency relations UP, RIGHT $\subseteq \mathcal{T}$ such that:

$$
\begin{aligned}
& \operatorname{UP}\left(t_{i}\right)=\bigvee\left\{t_{j} \mid \operatorname{up}_{T_{i}}=\operatorname{down}_{T_{j}}\right\}, \\
& \operatorname{RIGHT}\left(t_{i}\right)=\bigvee\left\{t_{j} \mid \operatorname{right}_{T_{i}}=\operatorname{left}_{T_{j}}\right\} .
\end{aligned}
$$

Then we pick $2 n$ propositional variables $p_{1}, \ldots p_{n}, q_{1}, \ldots, q_{n}$ and let $2^{n}=k$. We use them to encode a binary representation of coordinates of points in a grid. Namely, the truth values of $p_{i}$ and $q_{j}$ constitute bit vectors for corresponding coordinates of each point in our $k \times k$ grid. For example, if $k=8$, a pair of coordinates $(1,3)$ is encoded as $\left(\neg p_{3}, \neg p_{2}, p_{1}, \neg q_{3}, q_{2}, q_{1}\right)$, representing $(001,011)$.

So equipped, we can form a grid. All formulas that we use are presented in Fig. 3.

We start with a formula IP that forces the existence of the initial $(0,0)$ point of a grid.

Next, we ensure that each point in a model that has a pair of coordinates, has an appropriate number of successors, namely:

(i) both a right-adjacent and an up-adjacent successor with appropriate coordinates, if it is an internal point of a grid (formulas $\operatorname{lnc}_{0,0}, \operatorname{lnc}_{0, j}$, and Inc $_{i, j}$. A formula $\mathbf{I n c}_{i, 0}$, which is not explicitly formulated in Fig. 3, is defined in an analogous way to $\left.\operatorname{lnc}_{0, j}\right)$, 


\section{Grid formulas:}

$$
\begin{aligned}
& \mathrm{IP}:=\mathrm{E}_{=1}\left(\bigwedge_{i=1}^{n} \neg p_{i} \wedge \bigwedge_{j=1}^{n} \neg q_{j}\right)
\end{aligned}
$$

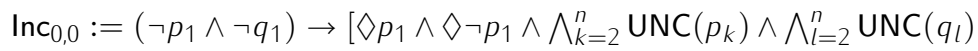

$$
\begin{aligned}
& \left.\wedge \square\left[\left(p_{1} \rightarrow \neg q_{1}\right) \wedge\left(\neg p_{1} \rightarrow q_{1}\right)\right]\right] \\
& \operatorname{Inc}_{0, j}:=\left(\neg p_{1} \wedge \neg q_{j+1} \wedge \bigwedge_{k=1}^{j} q_{k}\right) \rightarrow\left[\diamond p_{1} \wedge \diamond \neg p_{1} \wedge \bigwedge_{i=2}^{n} \operatorname{UNC}\left(p_{i}\right)\right. \\
& \wedge \bigwedge_{k=j+2}^{n} \operatorname{UNC}\left(q_{k}\right) \wedge \square\left[\left(p_{1} \rightarrow\left(\neg q_{j+1} \wedge \bigwedge_{k=1}^{j} q_{k}\right)\right)\right. \\
& \left.\left.\wedge\left(\neg p_{1} \rightarrow\left(q_{j+1} \wedge \wedge_{k=1}^{j} \neg q_{k}\right)\right)\right]\right], \quad 1 \leq j<n \\
& \operatorname{Inc}_{i, j}:=\left(\neg p_{i+1} \wedge \bigwedge_{k=1}^{i} p_{k} \wedge \neg q_{j+1} \wedge \bigwedge_{l=1}^{j} q_{l}\right) \rightarrow\left[\diamond p_{i+1} \wedge \diamond \neg p_{i+1}\right. \\
& \wedge \bigwedge_{k=i+2}^{n} \operatorname{UNC}\left(p_{k}\right) \wedge \bigwedge_{l=j+2}^{n} \operatorname{UNC}\left(q_{l}\right) \\
& \wedge \square\left[\left(p_{i+1} \rightarrow\left(\bigwedge_{k=1}^{i} \neg p_{k} \wedge \neg q_{j+1} \wedge \bigwedge_{l=1}^{j} q_{l}\right)\right)\right. \\
& \left.\left.\wedge\left(\neg p_{i+1} \rightarrow\left(\bigwedge_{k=1}^{i} p_{k} \wedge q_{j+1} \wedge \bigwedge_{l=1}^{j} \neg q_{l}\right)\right)\right]\right], \quad 0<i, j<n \\
& \operatorname{Inc}_{n, 0}:=\left(\bigwedge_{i=1}^{n} p_{i} \wedge \neg q_{1}\right) \rightarrow\left[\diamond \top \wedge \bigwedge_{j=2}^{n} \operatorname{UNC}\left(q_{j}\right) \wedge \square\left(\bigwedge_{i=1}^{n} p_{i} \wedge q_{1}\right)\right] \\
& \text { Inc }_{n, j}:=\left(\bigwedge_{i=1}^{n} p_{i} \wedge \neg q_{j+1} \wedge \bigwedge_{k=1}^{j} q_{k}\right) \rightarrow\left[\diamond \top \wedge \bigwedge_{k=j+2}^{n} \operatorname{UNC}\left(q_{k}\right)\right. \\
& \left.\wedge \square\left(\bigwedge_{i=1}^{n} p_{i} \wedge q_{j+1} \wedge \bigwedge_{k=1}^{j} \neg q_{k}\right)\right], \quad 1 \leq j<n \\
& \operatorname{lnc}_{n, n}:=\left(\bigwedge_{i=1}^{n} p_{i} \wedge \bigwedge_{j=1}^{n} q_{j}\right) \rightarrow \square \neg \top \\
& \text { Num }:=\mathrm{E}_{=2^{2 n}} \mathrm{~T}
\end{aligned}
$$

Tiling formulas:

$$
\begin{aligned}
\mathrm{Cov}:= & \bigvee_{i=0}^{m} t_{i} \\
\mathrm{Un}:= & \bigwedge_{0 \leq i<j \leq m}\left(t_{i} \rightarrow \neg t_{j}\right) \\
\mathrm{IPC}:= & \left(\bigwedge_{i=1}^{n} \neg p_{i} \wedge \bigwedge_{j=1}^{n} \neg q_{j}\right) \rightarrow t_{0} \\
\mathrm{Til}_{j}:= & t_{j} \rightarrow\left[\left[\neg p_{1} \rightarrow \square\left[\left(\neg p_{1} \rightarrow \operatorname{UP}\left(t_{j}\right)\right) \wedge\left(p_{1} \rightarrow \operatorname{RIGHT}\left(t_{j}\right)\right)\right]\right]\right. \\
& \wedge \bigwedge_{i=1}^{n-1}\left[( \neg p _ { i + 1 } \wedge \bigwedge _ { k = 1 } ^ { i } p _ { k } ) \rightarrow \square \left[\left(\neg p_{i+1} \rightarrow \operatorname{UP}\left(t_{j}\right)\right)\right.\right. \\
& \left.\left.\left.\wedge\left(p_{i+1} \rightarrow \operatorname{RIGHT}\left(t_{j}\right)\right)\right]\right] \wedge\left[\bigwedge_{i=1}^{n} p_{i} \rightarrow \square \operatorname{UP}\left(t_{j}\right)\right]\right], \quad 0 \leq j \leq m
\end{aligned}
$$

Figure 3: Formulas that encode a grid and a tiling. $\operatorname{Inc}_{0, j}, \operatorname{Inc}_{i, j}, \operatorname{Inc}_{n, j}$ and Til are formula-schemes with $i, j$ as parameters. 
(ii) either a right-adjacent or an up-adjacent successor, if it is an edge point of a grid (formulas $\operatorname{Inc}_{n, 0}$, Inc $c_{n, j}$. formulas $\operatorname{Inc}_{0, n}$, $\operatorname{Inc}_{i, n}$ are defined similarly to $\left.\operatorname{lnc}_{n, 0}, \ln c_{n, j}\right)$,

(iii) no successors if it is the end point of a grid (formula $I n c_{n, n}$ ).

To form these formulas, we use the two-dimensional version of the binary counter exploited in a proof of ExPTIME-completeness of PDL (cf. $[15,99])$. We abbreviate a formula $\left(p_{i} \rightarrow \square p_{i}\right) \wedge\left(\neg p_{i} \rightarrow \square \neg p_{i}\right)$ as $\operatorname{UNC}\left(p_{i}\right)$ and a formula $\left(q_{j} \rightarrow \square q_{j}\right) \wedge\left(\neg q_{j} \rightarrow \square \neg q_{j}\right)$ as $\operatorname{UNC}\left(q_{j}\right)$.

Together, the Inc formulas ensure that there exists at least $2^{2 n}$ distinct points in our model, each of which has a unique pair of coordinates. What is also secured is the adjacency conditions: each of the coordinated points has an up-adjacent and right-adjacent successor (with respect to the coordinates). However, apart from the $(k, k)$ point we have hitherto not ensured that each coordinated point has exactly the required number of successors (one or two). Moreover, commutativity of the up- and right-adjacency relations is not secured. Yet, if we constrain the cardinality of a model to $2^{2 n}$, it will mean that each pair of coordinates occurs only once and no other points are present in a model. Also, the commutativity condition will hold. We constrain the cardinality with the formula Num. Basically, what Num does is glue together all worlds with the same pairs of coordinates (cf. Fig. 4).

Now we are ready to define a formula that forms a grid-shaped model as:

$$
\text { Grid }:=\mathrm{IP} \wedge \mathrm{A}\left(\bigwedge_{i, j=0}^{n} \operatorname{lnc} c_{i, j}\right) \wedge \text { Num. }
$$

The initial segment of the resulting model looks as follows:

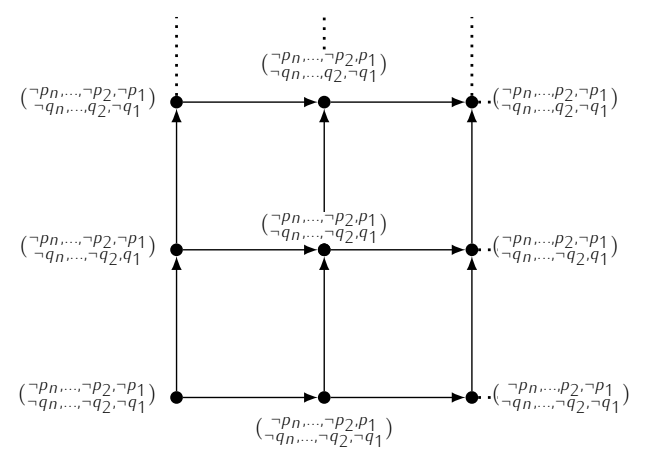




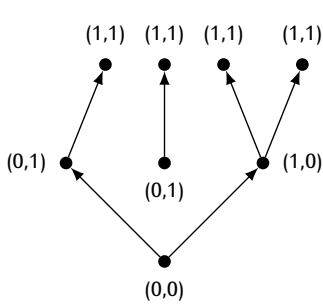

(a)

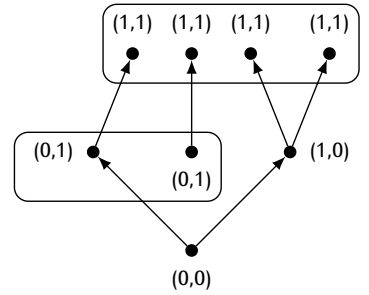

(b)

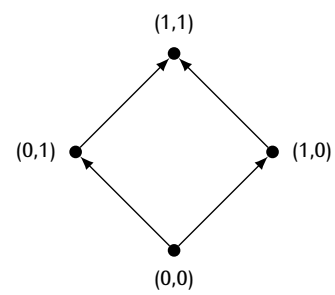

(c)

Figure 4: We consider a formula Grid for $n=1$. (a) presents one of the models satisfying IP $\wedge \mathrm{A}\left(\bigwedge_{i, j=0}^{1} \operatorname{lnc}_{i, j}\right)$ with no Num added (which is not grid-shaped), (b) shows that adding Num glues together all worlds with identical pairs of coordinates, (c) indicates that the unique shape of a model on which Grid is satisfied is grid.

Once forced into a grid-shaped model, we can define our tiling. All necessary tiling formulas are displayed in Fig. 3.

First, we need to ensure that our encoding of the tiling tiles all points of a grid and that not more than one tile type is matched with each point. We achieve it with formulas Cov and Un.

To accomplish the encoding of the tiling, we need to ensure satisfaction of the conditions (a)-(c) from Theorem 5.17. We obtain it by defining the formulas IPC and $\mathrm{Til}_{j}$.

A formula that fixes a tiling is defined as follows:

$$
\text { Tiling }:=\mathrm{A}\left(\operatorname{Cov} \wedge \mathrm{Un} \wedge \mathrm{IPC} \wedge \wedge_{j=0}^{m} \mathrm{Til}_{j}\right) .
$$

Now we can define our formula $\phi$ as Grid $\wedge$ Tiling.

We prove that (1) holds for $\phi$. For the left-to-right direction we assume that $\tau$ is a tiling for a $k \times k$ grid $\left(k=2^{n}\right)$ and a set of tile 
types $T=\left\{T_{0}, \ldots, T_{m}\right\}$. Given the sets of variables $\left\{p_{i}\right\}_{i=1}^{n},\left\{q_{i}\right\}_{i=1}^{n}$ and $\left\{t_{i}\right\}_{i=1}^{m}$ such that each $t_{i}$ is associated with $T_{i}$, we construct a model $\mathfrak{M}=\langle W, R, V\rangle$, where: $W=\left\{w_{i, j} \mid(i, j) \in k \times k\right\}, R=$ $\left\{\left(w_{i, j}, w_{s, t}\right) \in W^{2} \mid s=i+1, t=j\right.$ or $\left.s=i, t=j+1\right\}$, and the valuation of the propositional symbols is given by: $V\left(p_{i}\right)=\left\{w_{s, t} \in W \mid i\right.$-th bit of binary representation of $s$ is 1$\}, V\left(q_{i}\right)=\left\{w_{s, t} \in W \mid i\right.$-th bit of binary representation of $t$ is 1$\}$, and $V\left(t_{i}\right)=\left\{w_{s, t} \in W \mid \tau(s, t)=\right.$ $\left.T_{i}\right\}$. It is clear that $\phi$ is satisfied on $\mathfrak{M}$.

Now, the right-to-left direction. Suppose that $\mathfrak{M}$ satisfies $\phi$ for $\log (k)$ and $m$. From the construction of the formula Grid it follows that $\mathfrak{M}$ is grid-shaped. Let $w_{s, t}$ be a world in $\mathfrak{M}$ such that the formulas $p_{i}, q_{j}$ $(1 \leq i, j \leq n)$ are satisfied in $w_{s, t}$ in such a way that they form binary representations of $s$ and $t$ respectively. Next, we map $\mathfrak{M}$ to a grid via $f: W \longrightarrow k \times k$ such that $f\left(w_{s, t}\right)=(s, t)$. A function $\tau: k \times k \longrightarrow \mathrm{T}$ that satisfies the condition

$$
\tau(s, t)=T_{i} \quad \text { iff } \quad \mathfrak{M}, w_{s, t} \Vdash t_{i}
$$

is our tiling.

Even though $\phi$ has a complicated structure, it is a matter of routine to verify that it is computable in polynomial time in $m$ and $\log (k)$. Note that since number constraints are coded in binary, the actual size of the numerical subscript of the formula Num is $2 n$. This concludes the proof.

THEOREM 5.19. Modal logic with global counting operators defined over the class of reflexive frames $\left(\mathcal{M}_{\mathrm{T}}\left(\mathrm{E}_{n}\right)\right)$ is NEXPTIME-complete.

Proof. For the upper bound we exploit Theorem 5.16 and observe that a reduct of the initial, reflexive model, is also reflexive.

For the lower bound we employ the technique used for encoding the finite tiling problem in the logic $\mathcal{M}_{K}\left(E_{n}\right)$ in the proof of Theorem 5.17. However, it is easy to notice that due to the reflexivity it becomes impossible to shape a grid-shaped model as displayed in the proof of Lemma 5.17. It is therefore necessary to tamper a bit with the formulas encoding both, a grid- shaped model and a tiling (see Fig. 3). After certain modifications, the formula Grid $^{\text {Ref }}$, whose conjuncts are displayed 


\section{Grid $^{\text {Ref }}$ formulas:}

$$
\begin{aligned}
& \operatorname{Inc}_{0,0}^{\text {Ref }}:=\left(\neg p_{1} \wedge \neg q_{1}\right) \rightarrow\left[\diamond\left(\neg p_{1} \wedge \neg q_{1}\right) \wedge \diamond\left(\neg p_{1} \wedge q_{1}\right) \wedge \diamond p_{1} \wedge \wedge_{i=2}^{n} \operatorname{UNC}\left(p_{i}\right)\right. \\
& \left.\wedge \wedge_{j=2}^{n} \operatorname{UNC}\left(q_{j}\right) \wedge \square\left[\left(p_{1} \rightarrow \neg q_{1}\right)\right]\right] \\
& \operatorname{Inc}_{0, j}^{\text {Ref }}:=\left(\neg p_{1} \wedge \neg q_{j+1} \wedge \wedge_{k=1}^{j} q_{k}\right) \rightarrow\left[\diamond\left(\neg p_{1} \wedge \neg q_{j+1}\right)\right. \\
& \wedge \diamond\left(\neg p_{1} \wedge q_{j+1}\right) \wedge \diamond p_{1} \wedge \bigwedge_{i=2}^{n} \operatorname{UNC}\left(p_{i}\right) \wedge \bigwedge_{k=j+2}^{n} \operatorname{UNC}\left(q_{k}\right) \\
& \wedge \square\left[\left(\left(\neg p_{1} \neg q_{j+1}\right) \rightarrow\left(\bigwedge_{k=1}^{j} q_{k}\right)\right) \wedge\left(\left(\neg p_{1} \wedge q_{j+1}\right) \rightarrow\left(\bigwedge_{k=1}^{j} \neg q_{k}\right)\right)\right. \\
& \left.\left.\wedge\left(p_{1} \rightarrow\left(\neg q_{j+1} \wedge \wedge_{k=1}^{j} q_{k}\right)\right)\right]\right], \quad 1 \leq j<n \\
& \operatorname{Inc}_{i, j}^{\text {Ref }}:=\left(\neg p_{i+1} \wedge \bigwedge_{k=1}^{i} p_{k} \wedge \neg q_{j+1} \wedge \bigwedge_{l=1}^{j} q_{l}\right) \rightarrow\left[\diamond\left(\neg p_{i+1} \wedge \neg q_{j+1}\right)\right. \\
& \wedge \diamond\left(\neg p_{i+1} \wedge q_{j+1}\right) \wedge \diamond p_{i+1} \wedge \wedge_{k=i+2}^{n} \operatorname{UNC}\left(p_{k}\right) \wedge \bigwedge_{l=j+2}^{n} \operatorname{UNC}\left(q_{l}\right) \\
& \wedge \square\left[\left(\left(\neg p_{i+1} \wedge \neg q_{j+1}\right) \rightarrow\left(\bigwedge_{k=1}^{i} p_{k} \wedge \bigwedge_{l=1}^{j} q_{l}\right)\right)\right. \\
& \wedge\left(\left(\neg p_{i+1} \wedge q_{j+1}\right) \rightarrow\left(\bigwedge_{k=1}^{i} p_{k} \wedge \bigwedge_{l=1}^{j} \neg q_{l}\right)\right) \\
& \left.\left.\wedge\left(p_{i+1} \rightarrow\left(\bigwedge_{k=1}^{i} \neg p_{k} \wedge \neg q_{j+1} \wedge \bigwedge_{l=1}^{j} q_{l}\right)\right)\right]\right], \quad 0<i, j<n \\
& \operatorname{Inc}_{n, 0}^{\text {Ref }}:=\left(\bigwedge_{i=1}^{n} p_{i} \wedge \neg q_{1}\right) \rightarrow\left[\diamond \neg q_{1} \wedge \diamond q_{1} \wedge \bigwedge_{i=1}^{n} \operatorname{UNC}\left(p_{i}\right) \wedge \bigwedge_{j=2}^{n} \operatorname{UNC}\left(q_{j}\right)\right] \\
& \operatorname{Inc}_{n, j}^{\text {Ref }}:=\left(\bigwedge_{i=1}^{n} p_{i} \wedge \neg q_{j+1} \wedge \bigwedge_{k=1}^{j} q_{k}\right) \rightarrow\left[\diamond \neg q_{j+1} \wedge \diamond q_{j+1}\right. \\
& \wedge \bigwedge_{i=1}^{n} \operatorname{UNC}\left(p_{i}\right) \wedge \bigwedge_{k=j+2}^{n} \operatorname{UNC}\left(q_{k}\right) \wedge \square\left[\left(\neg q_{j+1} \wedge \bigwedge_{k=1}^{j} q_{k}\right)\right. \\
& \left.\left.\wedge\left(q_{j+1} \wedge \wedge_{k=1}^{j} \neg q_{k}\right)\right]\right], \quad 1 \leq j<n \\
& \operatorname{Inc}_{n, n}^{\text {Ref }}:=\left(\bigwedge_{i=1}^{n} p_{i} \wedge \bigwedge_{j=1}^{n} q_{j}\right) \rightarrow \square\left(\bigwedge_{i=1}^{n} p_{i} \wedge \bigwedge_{j=1}^{n} q_{j}\right)
\end{aligned}
$$

Tiling $^{\text {Ref }}$ formulas:

$$
\begin{aligned}
& \mathrm{Til}_{j}^{\text {Ref }}:=t_{j} \rightarrow\left[\left[\left(\neg p_{1} \wedge \neg q_{1}\right) \rightarrow \square\left[\left(\left(\neg p_{1} \wedge q_{1}\right) \rightarrow \mathrm{UP}\left(t_{j}\right) \wedge\left(p_{1} \rightarrow \operatorname{RIGHT}\left(t_{j}\right)\right)\right]\right]\right.\right. \\
& \wedge \bigwedge_{j=1}^{n-1}\left[( \neg p _ { 1 } \wedge \neg q _ { j + 1 } \wedge \bigwedge _ { k = 1 } ^ { j } q _ { k } ) \rightarrow \square \left[\left(\left(\neg p_{1} \wedge q_{j+1}\right) \rightarrow \operatorname{UP}\left(t_{j}\right)\right)\right.\right. \\
& \left.\left.\wedge\left(p_{1} \rightarrow \operatorname{RIGHT}\left(t_{j}\right)\right)\right]\right] \\
& \wedge\left[\left(\neg p_{1} \wedge \bigwedge_{j=1}^{n} q_{j}\right) \rightarrow \square\left(p_{1} \rightarrow \operatorname{RIGHT}\left(t_{j}\right)\right)\right] \\
& \wedge \bigwedge_{i=1}^{n-1}\left[( \neg p _ { i + 1 } \wedge \bigwedge _ { k = 1 } ^ { i } p _ { k } \wedge \neg q _ { 1 } ) \rightarrow \square \left[\left(\left(\neg p_{i+1} \wedge q_{1}\right) \rightarrow \operatorname{UP}\left(t_{j}\right)\right)\right.\right. \\
& \left.\wedge\left(p_{i+1} \rightarrow \operatorname{RIGHT}\left(t_{j}\right)\right]\right] \\
& \wedge \bigwedge_{i, j=1}^{n-1}\left[\left(\neg p_{i+1} \wedge \bigwedge_{k=1}^{i} p_{k} \wedge \neg q_{j+1} \wedge \bigwedge_{l=1}^{j} q_{l}\right)\right. \\
& \rightarrow \square\left[\left(\left(\neg p_{i+1} \wedge q_{j+1}\right) \rightarrow \operatorname{UP}\left(t_{j}\right) \wedge\left(p_{i+1} \rightarrow \operatorname{RIGHT}\left(t_{j}\right)\right)\right]\right] \\
& \wedge \bigwedge_{i=1}^{n-1}\left[\left(\neg p_{i+1} \wedge \bigwedge_{k=1}^{i} p_{k} \wedge \bigwedge_{j=1}^{n} q_{j}\right) \rightarrow \square\left(p_{i+1} \rightarrow \operatorname{RIGHT}\left(t_{j}\right)\right)\right] \\
& \wedge\left[\left(\bigwedge_{i=1}^{n} p_{i} \wedge \neg q_{1}\right) \rightarrow \square\left(q_{1} \rightarrow \operatorname{UP}\left(t_{j}\right)\right)\right] \\
& \left.\wedge \bigwedge_{j=1}^{n-1}\left[\left(\bigwedge_{i=1}^{n} p_{i} \wedge \neg q_{j+1} \wedge \bigwedge_{k=1}^{j} q_{k}\right) \rightarrow \square\left(q_{j+1} \rightarrow \mathrm{UP}\left(t_{j}\right)\right)\right]\right], \quad 0 \leq j \leq m
\end{aligned}
$$

Figure 5: Formulas that encode a grid and a tiling over reflexive frames. 
in Fig. $5^{6}$, enables us to form a model whose shape is very close to grid. It is visible that a model forced by Grid $^{\text {Ref }}$ is reflexive as exhibited in the following picture:

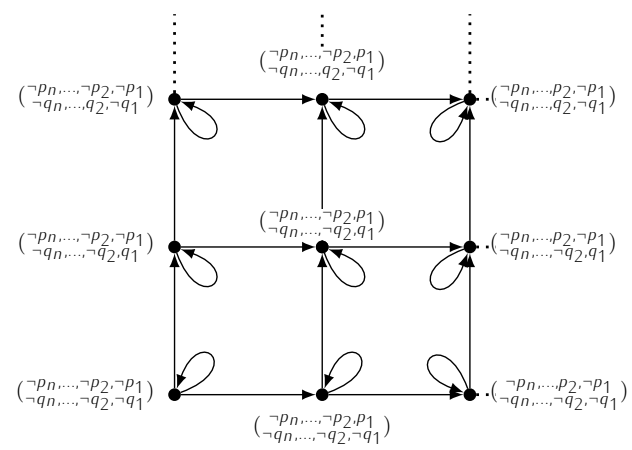

Tiling $^{\text {Ref }}$ encodes a tiling for a model tailored in this fashion.

Finally, Grid ${ }^{\text {Ref }} \wedge$ Tiling $^{\text {Ref }}$ is computable in polynomial time in $n$ and $m$, whence the conclusion follows.

THEOREM 5.20. Modal logic with global counting operators defined over the class of symmetric frames $\left(\mathcal{M}_{\mathrm{B}}\left(\mathrm{E}_{n}\right)\right)$ is NEXPTIME-complete.

Proof. For the upper bound, we suitably tamper with Theorem 5.16. Suppose that $\mathfrak{M}$ is a model for a formula $\varphi$. We construct a reduct $\mathfrak{M}^{\prime}=\left\langle W^{\prime}, R^{\prime}, V^{\prime}\right\rangle$ of a $\mathfrak{M}$ in the way described in the first part of the indicated theorem. Let $R^{\prime *}$ be a symmetric closure of $R$. We show that $\mathfrak{M}^{\prime *}=\left\langle W^{\prime}, R^{\prime *}, V^{\prime}\right\rangle$ is a model for $\varphi$, i.e. $R^{\prime *}$ is of the form $R^{\prime *}=R^{\prime} \cup$ $\left\{\langle w, v\rangle \mid\langle v, w\rangle \in R^{\prime}\right\}$. We first observe that closing the reduct under symmetry of the accessibility relation does not violate the satisfaction conditions for $\mathrm{E}_{<n}$ subformulas of the initial formula since by that means we do not increase the global number of accessibility arrows (and worlds satisfying, e.g., $\diamond T$ ) since each arrow removed by constructing $\mathfrak{M}^{\prime}$ was also symmetric. Now, the only thing that was jeopardized by adding symmetric complements to arrows was the satisfaction of modal formulas $\nabla \psi$. Let $\langle w, v\rangle \in R^{\prime}$ and $\langle v, w\rangle \notin R^{\prime}$ (but $\langle v, w\rangle \in R^{* *}$ ).

6 Figures only contain these formulas that require reformulating, relatively to the initial Grid and Tiling formulas. The remaining conjuncts are left unchanged. It therefore means that we obtain the formulas Grid ${ }^{\text {Frame }} \wedge$ Tiling ${ }^{\text {Frame }}$ by replacing appropriate conjuncts in the formula Grid $\wedge$ Tiling. 
Suppose that for some $\nabla \psi \in \operatorname{Sub}(\varphi) \mathfrak{M}^{*}, v \Vdash \neg \nabla \psi$ and $\mathfrak{M}^{*}, w \Vdash \psi$. Since $\langle v, w\rangle \notin R^{\prime}$, it follows that $\langle w, v\rangle \notin R$. For suppose otherwise. By symmetry of $R$, the fact that $w, v \in W^{\prime}$ and $\langle w, v\rangle \in R$, we would obtain $\langle v, w\rangle \in R^{\prime}$ and it is not the case. By construction of $\mathfrak{M}^{\prime}$ there exists $z$ such that $z{ }^{m} \operatorname{Sub}(\varphi) v$ and $\langle w, z\rangle \in R$. By symmetry of $R$, $\langle z, w\rangle \in R$. $v$ and $z$ satisfy the same formulas from $\operatorname{Sub}(\varphi)$, so by the assumption, $\mathfrak{M}, z \Vdash \neg \diamond \psi$. But that would contradict the consistency of $\mathfrak{M}$. Hence, the conclusion.

For the lower bound, again, we modify the formula Grid $\wedge$ Tiling obtaining Grid ${ }^{\text {Sym }} \wedge$ Tiling ${ }^{\text {Sym }}$.

However, since worlds in this model can have at most 4, and not 2 , successors, there occurs a necessity to re-categorise worlds in a model according to the "character" of their coordinates. We therefore distinguish two types of points within the category labelled formerly by 0 . The worlds which are not the "edge" worlds but whose coordinate's first digit equals 0 (which means that the formula $\neg p_{1}$ holds at this world) are named 0 worlds. The "edge" worlds in a coordinate of which every digit equals 0 is labelled by $0 *$. We need to distinguish between the two, because the former can have even 4 successors, whereas the latter at most three (in the case when a considered world is located "on the edge" of a grid but in neither of its corners. What follows, the formula Grid itself for symmetric frames needs a tiny reformulation in a following manner:

$$
\begin{aligned}
& \operatorname{Grid}^{\text {Sym }}:=\mathrm{IP} \wedge \mathrm{A}\left(\bigwedge_{i, j=0}^{n} \operatorname{Inc}_{i, j}^{\text {Sym }} \wedge \operatorname{Inc} c_{0 *, 0 *}^{\text {Sym }}\right. \\
& \left.\wedge \bigwedge_{i=0}^{n} \operatorname{Inc}_{i, 0 *}^{\text {Sym }} \wedge \bigwedge_{j=0}^{n} \operatorname{Inc}_{0 *, j}^{\text {Sym }}\right) \wedge \text { Num. }
\end{aligned}
$$

The resulting frame has a form of a "symmetric grid" 


$$
\begin{aligned}
& \text { Grid }^{\text {Sym }} \text { formulas: } \\
& \operatorname{Inc}_{0 *, 0 *}^{\mathrm{Sym}}:=\left(\bigwedge_{i=1}^{n} \neg p_{i} \wedge \bigwedge_{j=1}^{n} \neg q_{j}\right) \rightarrow\left[\diamond p_{1} \wedge \diamond \neg p_{1} \wedge \bigwedge_{i=2}^{n} \operatorname{UNC}\left(p_{i}\right)\right. \\
& \left.\wedge \wedge_{j=2}^{n} \operatorname{UNC}\left(q_{j}\right) \wedge \square\left(p_{1} \leftrightarrow \neg q_{1}\right)\right] \\
& \operatorname{Inc}_{0 *, 0}^{\mathrm{Sym}}:=\left(\bigwedge_{i=1}^{n} \neg p_{1} \wedge q_{j+1} \wedge \bigwedge_{k=1}^{j} \neg q_{k}\right) \rightarrow\left[\diamond p_{1} \wedge \diamond\left(\neg p_{1} \wedge q_{1}\right)\right. \\
& \wedge \diamond\left(\neg p_{1} \wedge \neg q_{j+1}\right) \wedge \bigwedge_{i=2}^{n} \operatorname{UNC}\left(p_{i}\right) \wedge \bigwedge_{l=j+2}^{n} \operatorname{UNC}\left(q_{l}\right) \\
& \wedge \square\left[\left(\left(\left(p_{1} \rightarrow\left(q_{j+1} \wedge \wedge_{k=1}^{j} \neg q_{k}\right)\right) \wedge\left(\left(\neg p_{1} \wedge q_{1}\right) \rightarrow\left(q_{j+1} \wedge \bigwedge_{k=1}^{j} \neg q_{j}\right)\right)\right.\right.\right. \\
& \left.\left.\wedge\left(\left(\neg p_{1} \wedge \neg q_{j+1}\right) \rightarrow\left(\bigwedge_{k=1}^{j} q_{k}\right)\right)\right]\right], \quad 1 \leq j<n \\
& \operatorname{Inc}_{0,0}^{\text {Sym }}:=\left(p_{i+i} \wedge \bigwedge_{k=1}^{i} \neg p_{k} \wedge q_{j+1} \wedge \wedge_{l=1}^{j}\right) \rightarrow\left[\diamond\left(\neg p_{1} \wedge q_{1}\right) \wedge \diamond\left(\neg p_{1} \wedge \neg q_{j+1}\right)\right. \\
& \wedge \diamond\left(p_{1} \wedge \neg q_{1}\right) \wedge \diamond \neg p_{i+1} \wedge \bigwedge_{k=i+2}^{n} \operatorname{UNC}\left(p_{k}\right) \wedge \bigwedge_{l=j+2}^{n} \operatorname{UNC}\left(q_{l}\right) \\
& \wedge \square\left[\left(\left(\neg p_{1} \wedge q_{1}\right) \rightarrow\left(p_{i+1} \wedge \bigwedge_{k=2}^{i} \neg p_{k} \wedge q_{j+1} \wedge \bigwedge_{l=2}^{j} \neg q_{l}\right)\right)\right. \\
& \wedge\left(\left(\neg p_{1} \wedge \neg q_{j+1}\right) \rightarrow\left(p_{i+1} \wedge \bigwedge_{k=2}^{i} \neg p_{k} \wedge \bigwedge_{l=1}^{j} q_{l}\right)\right) \\
& \wedge\left(\left(p_{1} \wedge \neg q_{1}\right) \rightarrow\left(p_{i+1} \wedge \bigwedge_{k=2}^{i} \neg p_{k} \wedge q_{j+1} \wedge \bigwedge_{l=2}^{j} \neg q_{l}\right)\right) \\
& \left.\left.\wedge\left(\neg p_{i+1} \rightarrow\left(\bigwedge_{k=1}^{i} p_{k} \wedge q_{j+1} \wedge \bigwedge_{l=1}^{j} \neg q_{l}\right)\right)\right]\right], \quad 0<i, j<n \\
& \operatorname{Inc}_{0 *, j}^{\mathrm{Sym}}:=\left(\bigwedge_{i=1}^{n} \neg p_{i} \wedge \neg q_{j+1} \wedge \bigwedge_{k=1}^{j} q_{k}\right) \rightarrow\left[\diamond p_{1} \wedge \diamond\left(\neg p_{1} \wedge \neg q_{1}\right) \wedge \diamond\left(\neg p_{1} \wedge q_{j+1}\right)\right. \\
& \wedge \bigwedge_{i=2}^{n} \operatorname{UNC}\left(p_{i}\right) \wedge \bigwedge_{k=j+2}^{n} \operatorname{UNC}\left(q_{j}\right) \wedge \square\left[\left(p_{1} \rightarrow\left(\neg q_{j+1} \wedge \bigwedge_{k=1}^{j} q_{k}\right)\right)\right. \\
& \wedge\left(\left(\neg p_{1} \wedge \neg q_{1}\right) \rightarrow\left(\neg q_{j+1} \wedge \wedge_{k=2}^{j} q_{k}\right)\right) \\
& \left.\left.\wedge\left(\left(\neg p_{1} \wedge q_{j+1}\right) \rightarrow \bigwedge_{k=1}^{j} \neg q_{k}\right)\right]\right], \quad 0<j<n \\
& \operatorname{Inc}_{0, j}^{\text {Sym }}:=\left(p_{i+1} \wedge \bigwedge_{k=1}^{i} \neg p_{k} \wedge \neg q_{j+1} \wedge \bigwedge_{l=1}^{j} q_{l}\right) \rightarrow\left[\diamond\left(p_{1} \wedge p_{i+1}\right) \wedge \diamond\left(p_{1} \wedge \neg p_{i+1}\right)\right. \\
& \wedge \diamond\left(\neg p_{1} \wedge \neg q_{j+1}\right) \wedge \diamond\left(\neg p_{1} \wedge q_{j+1}\right) \wedge \wedge_{k=i+2}^{n} \operatorname{UNC}\left(p_{k}\right) \wedge \bigwedge_{l=j+2}^{n} \operatorname{UNC}\left(q_{l}\right) \\
& \wedge \square\left[\left(\left(p_{1} \wedge p_{i+1}\right) \rightarrow\left(\bigwedge_{k=1}^{n} \neg p_{k} \wedge \neg q_{j+1} \wedge \bigwedge_{l=1}^{j} q_{l}\right)\right)\right. \\
& \wedge\left(\left(p_{1} \wedge \neg p_{i+1}\right) \rightarrow\left(\bigwedge_{k=1}^{i} p_{k} \wedge \neg q_{j+1} \wedge \bigwedge_{l=1}^{j} q_{l}\right)\right) \\
& \wedge\left(\left(\neg p_{1} \wedge \neg q_{j+1}\right) \rightarrow\left(p_{i+1} \wedge \bigwedge_{k=1}^{i} \neg p_{k} \wedge \neg q_{j+1} \wedge \bigwedge_{l=1}^{j} q_{l}\right)\right) \\
& \left.\left.\wedge\left(\left(\neg p_{1} \wedge q_{j+1}\right) \rightarrow\left(p_{i+1} \wedge \bigwedge_{k=1}^{i} \neg p_{k} \wedge \bigwedge_{l=1}^{j} \neg q_{l}\right)\right)\right]\right], \quad 1 \leq j<n
\end{aligned}
$$

Figure 6: Formulas that encode a grid and a tiling over symmetric frames. 


$$
\begin{aligned}
& \operatorname{Inc}_{i, j}^{\text {Sym }}:=\left(\neg p_{i+1} \wedge \bigwedge_{k=1}^{i} p_{k} \wedge \neg q_{j+1} \wedge \bigwedge_{l=1}^{j} q_{j}\right) \rightarrow\left[\diamond\left(\neg p_{1} \wedge \neg p_{i+1}\right)\right. \\
& \wedge \diamond\left(\neg p_{1} \wedge p_{i+1}\right) \wedge \diamond\left(p_{1} \wedge q_{j+1}\right) \wedge \diamond\left(p_{1} \wedge \neg q_{j+1}\right) \wedge \bigwedge_{k=i+2}^{n} \operatorname{UNC}\left(p_{k}\right) \\
& \wedge \bigwedge_{l=j+2}^{n} \operatorname{UNC}\left(q_{l}\right) \wedge \square\left[\left(\left(\neg p_{1} \wedge \neg p_{i+1}\right) \rightarrow\left(\bigwedge_{k=2}^{i} p_{k} \wedge \neg q_{j+1} \wedge \bigwedge_{l=1}^{j} q_{l}\right)\right)\right. \\
& \wedge\left(\left(\neg p_{1} \wedge p_{i+1}\right) \rightarrow\left(\bigwedge_{k=2}^{i} \neg p_{k} \wedge \neg q_{j+1} \wedge \bigwedge_{l=1}^{j} q_{l}\right)\right) \\
& \wedge\left(\left(p_{1} \wedge q_{j+1}\right) \rightarrow\left(\neg p_{i+1} \wedge \bigwedge_{k=2}^{i} p_{k} \wedge \bigwedge_{l=1}^{j} \neg q_{l}\right)\right) \\
& \left.\left.\wedge\left(\left(p_{1} \wedge \neg q_{j+1}\right) \rightarrow\left(\neg p_{i+1} \wedge \bigwedge_{k=2}^{i} p_{k} \wedge \neg q_{1} \wedge \bigwedge_{l=2}^{j} q_{l}\right)\right)\right]\right], \quad 1 \leq i, j<n \\
& \operatorname{Inc}_{n, 0 *}^{\mathrm{Sym}}:=\left(\bigwedge_{i=1}^{n} p_{i} \wedge \bigwedge_{j=1}^{n} \neg q_{j}\right) \rightarrow\left[\diamond p_{1} \wedge \neg p_{1} \wedge \bigwedge_{i=2}^{n} \operatorname{UNC}\left(p_{i}\right) \wedge \bigwedge_{j=2}^{n} \operatorname{UNC}\left(q_{j}\right)\right. \\
& \left.\wedge \square\left(p_{1} \leftrightarrow q_{1}\right)\right] \\
& \operatorname{Inc}_{n, 0}^{\text {Sym }}:=\left(\bigwedge_{i=1}^{n} p_{i} \wedge q_{j+1} \wedge \bigwedge_{k=1}^{j} \neg q_{k}\right) \rightarrow\left[\diamond \neg p_{1} \wedge \diamond\left(p_{1} \wedge q_{1}\right)\right. \\
& \wedge \diamond\left(p_{1} \wedge \neg q_{j+1}\right) \wedge \bigwedge_{i=2}^{n} \operatorname{UNC}\left(p_{i}\right) \wedge \bigwedge_{k=j+2}^{n} \operatorname{UNC}\left(q_{k}\right) \\
& \wedge \square\left[\left(\neg p_{1} \rightarrow\left(q_{j+1} \wedge \bigwedge_{k=1}^{j} \neg q_{k}\right)\right) \wedge\left(\left(p_{1} \wedge q_{1}\right) \rightarrow\left(q_{j+1} \wedge \bigwedge_{k=2}^{j} \neg q_{k}\right)\right)\right. \\
& \left.\left.\wedge\left(\left(p_{1} \wedge \neg q_{j+1}\right) \rightarrow \bigwedge_{k=1}^{j} q_{k}\right)\right]\right], \quad 1 \leq i, j<n \\
& \operatorname{Inc}_{n, j}^{\text {Sym }}:=\left(\bigwedge_{i=1}^{n} p_{i} \wedge \neg q_{j+1} \wedge \bigwedge_{k=1}^{j} q_{k}\right) \rightarrow\left[\diamond \neg p_{1} \wedge \diamond\left(p_{1} \wedge q_{j+1}\right)\right. \\
& \wedge \diamond\left(p_{1} \wedge \neg q_{j+1}\right) \wedge \bigwedge_{i=2}^{n} \operatorname{UNC}\left(p_{i}\right) \wedge \bigwedge_{k=j+2}^{n} \operatorname{UNC}\left(q_{k}\right) \\
& \wedge \square\left[\left(\neg p_{1} \rightarrow\left(\neg q_{j+1} \wedge \wedge_{k=1}^{j} q_{k}\right)\right) \wedge\left(\left(p_{1} \wedge q_{j+1}\right) \rightarrow \bigwedge_{k=1}^{j} \neg q_{k}\right)\right. \\
& \left.\left.\wedge\left(\left(p_{1} \wedge \neg q_{j+1}\right) \rightarrow\left(\neg q_{1} \wedge_{k=2}^{j} q_{k}\right)\right)\right]\right], \quad 1 \leq j<n \\
& \operatorname{Inc}_{n, n}^{\text {Sym }}:=\left(\bigwedge_{i=1}^{n} p_{i} \wedge \bigwedge_{j=1}^{n} q_{j}\right) \rightarrow\left[\diamond \neg p_{1} \wedge \diamond p_{1} \wedge \bigwedge_{i=2}^{n} \operatorname{UNC}\left(p_{i}\right) \wedge \bigwedge_{j=2}^{n} \operatorname{UNC}\left(q_{j}\right)\right. \\
& \left.\wedge \square\left(\neg p_{1} \leftrightarrow q_{1}\right)\right]
\end{aligned}
$$

Continued Figure 6: Formulas that encode a grid and a tiling over symmetric frames. 
Tiling Sym formulas:

$$
\begin{aligned}
& \mathrm{Til}_{j}^{\text {Sym }}:=t_{j} \rightarrow\left[\left[( \bigwedge _ { i = 1 } ^ { n } \neg p _ { i } \wedge \bigwedge _ { j = 1 } ^ { n } \neg q _ { j } ) \rightarrow \square \left[\left(\neg p_{1} \rightarrow \operatorname{UP}\left(t_{j}\right)\right.\right.\right.\right. \\
& \left.\left.\wedge\left(p_{1} \rightarrow \operatorname{RIGHT}\left(t_{j}\right)\right)\right]\right] \\
& \wedge \bigwedge_{j=1}^{n-1}\left[( \bigwedge _ { i = 1 } ^ { n } \neg p _ { i } \wedge q _ { j + 1 } \wedge \bigwedge _ { k = 1 } ^ { j } \neg q _ { k } ) \rightarrow \square \left[\left(\left(\neg p_{1} \wedge q_{j+1}\right) \rightarrow \operatorname{UP}\left(t_{j}\right)\right)\right.\right. \\
& \left.\left.\wedge\left(p_{1} \rightarrow \operatorname{RIGHT}\left(t_{j}\right)\right)\right]\right] \\
& \wedge \bigwedge_{j=1}^{n-1}\left[\left(\bigwedge_{i=1}^{n} \neg p_{i} \wedge \neg q_{j+1} \bigwedge_{k=1}^{n} q_{k}\right) \rightarrow \square\left[\left(\neg p_{1} \wedge q_{j+1}\right) \rightarrow \operatorname{UP}\left(t_{j}\right)\right)\right. \\
& \left.\left.\wedge\left(p_{1} \rightarrow \operatorname{RIGHT}\left(t_{j}\right)\right)\right]\right] \\
& \wedge\left[\left(\bigwedge_{i=1}^{n} \neg p_{i} \wedge \bigwedge_{j=1}^{n} q_{j}\right) \rightarrow \square\left(p_{1} \rightarrow \operatorname{RIGHT}\left(t_{j}\right)\right)\right] \\
& \wedge \bigwedge_{i=1}^{n-1}\left[( p _ { i + 1 } \wedge \bigwedge _ { k = 1 } ^ { i } \neg p _ { k } \wedge \bigwedge _ { j = 1 } ^ { n } \neg q _ { j } ) \rightarrow \square \left[\left(q_{1} \rightarrow \operatorname{UP}\left(t_{j}\right)\right)\right.\right. \\
& \left.\left.\wedge\left(\left(p_{i+1} \wedge \neg q_{1}\right) \rightarrow \operatorname{RIGHT}\left(t_{j}\right)\right)\right]\right] \\
& \wedge \bigwedge_{i, j=1}^{n-1}\left[\left(p_{i+1} \wedge \bigwedge_{k=1}^{i} \neg p_{k} \wedge q_{j+1} \wedge \bigwedge_{l=1}^{j} \neg q_{j}\right)\right. \\
& \left.\rightarrow \square\left[\left(\left(\neg p_{1} \wedge q_{j+1}\right) \rightarrow \mathrm{UP}\left(t_{j}\right)\right) \wedge\left(\left(p_{i+1} \wedge \neg q_{1}\right) \rightarrow \operatorname{RIGHT}\left(t_{j}\right)\right)\right]\right] \\
& \wedge \bigwedge_{i, j=1}^{n-1}\left[\left(p_{i+1} \wedge \bigwedge_{k=1}^{i} \neg p_{i} \wedge \neg q_{j+1} \wedge \bigwedge_{l=1}^{j} q_{l}\right)\right. \\
& \left.\rightarrow \square\left[\left(\left(\neg p_{1} \wedge q_{j+1}\right) \rightarrow \mathrm{UP}\left(t_{j}\right)\right) \wedge\left(\left(p_{i+1} \wedge q_{1}\right) \rightarrow \operatorname{RIGHT}\left(t_{j}\right)\right)\right]\right] \\
& \wedge \bigwedge_{i=1}^{n-1}\left[\left(p_{i+1} \wedge \bigwedge_{k=1}^{i} \neg p_{k} \wedge \bigwedge_{j=1}^{n} q_{j}\right) \rightarrow \square\left(\left(p_{1} \wedge p_{i+1}\right) \rightarrow \operatorname{RIGHT}\left(t_{j}\right)\right)\right] \\
& \wedge \bigwedge_{i=1}^{n-1}\left[( \neg p _ { i + 1 } \wedge \bigwedge _ { k = 1 } ^ { i } p _ { k } \wedge \bigwedge _ { j = 1 } ^ { n } \neg q _ { j } ) \rightarrow \square \left[\left(q_{1} \rightarrow \mathrm{UP}\left(t_{j}\right)\right)\right.\right. \\
& \left.\left.\wedge\left(p_{i+1} \rightarrow \operatorname{RIGHT}\left(t_{j}\right)\right)\right]\right] \\
& \wedge \bigwedge_{i, j=1}^{n-1}\left[\left(\neg p_{i+1} \wedge \bigwedge_{k=1}^{i} p_{k} \wedge q_{j+1} \wedge \bigwedge_{l=1}^{j} \neg q_{l}\right)\right. \\
& \left.\rightarrow \square\left[\left(\left(q_{1} \wedge q_{i+1}\right) \rightarrow \mathrm{UP}\left(t_{j}\right)\right) \wedge\left(p_{i+1} \rightarrow \operatorname{RIGHT}\left(t_{j}\right)\right)\right]\right] \\
& \wedge \bigwedge_{i, j=1}^{n-1}\left[\left(\neg p_{i+1} \wedge \bigwedge_{k=1}^{i} p_{k} \wedge \neg q_{j+1} \wedge \bigwedge_{l=1}^{j} q_{l}\right)\right. \\
& \left.\rightarrow \square\left[\left(q_{j+1} \rightarrow \mathrm{UP}\left(t_{j}\right)\right) \wedge\left(p_{i+1} \rightarrow \operatorname{RIGHT}\left(t_{j}\right)\right)\right]\right] \\
& \wedge \bigwedge_{i=1}^{n-1}\left[\left(\neg p_{i+1} \wedge \bigwedge_{k=1}^{i} p_{k} \wedge \bigwedge_{j=1}^{n} q_{j}\right) \rightarrow \square\left(p_{i+1} \rightarrow \operatorname{RIGHT}\left(t_{j}\right)\right)\right] \\
& \wedge\left[\left(\bigwedge_{i=1}^{n} p_{k} \wedge \bigwedge_{j=1}^{n} q_{j}\right) \rightarrow \square\left(q_{1} \rightarrow \operatorname{UP}\left(t_{j}\right)\right)\right] \\
& \wedge \bigwedge_{j=1}^{n-1}\left[\left(\bigwedge_{i=1}^{n} p_{k} \wedge q_{j+1} \wedge \bigwedge_{l=1}^{j} \neg q_{l}\right) \rightarrow \square\left(\left(q_{1} \wedge q_{j+1}\right) \rightarrow \operatorname{RIGHT}\left(t_{j}\right)\right)\right] \\
& \wedge ! \bigwedge_{j=1}^{n-1}\left[\left(\bigwedge_{i=1}^{n} p_{k} \wedge \neg q_{j+1} \wedge \bigwedge_{k=1}^{j} q_{k}\right) \rightarrow \square\left(q_{j+1} \rightarrow \mathrm{UP}\left(t_{j}\right)\right)\right], \quad 0 \leq j \leq m
\end{aligned}
$$

Continued Figure 6: Formulas that encode a grid and a tiling over symmetric frames. 
Grid $^{\text {Ser formuls: }}$

$\operatorname{Inc}_{n, n}^{\text {Ser }}:=\left(\bigwedge_{i=1}^{n} p_{i} \wedge \bigwedge_{j=1}^{n} q_{j}\right) \rightarrow \square\left(\bigwedge_{i=1}^{n} p_{i} \wedge \bigwedge_{j=1}^{n} q_{j}\right)$

Tiling ${ }^{\text {Ser }}$ formuls:

$$
\begin{aligned}
\operatorname{Til}_{j}^{\text {Ser }}:= & t_{j} \rightarrow\left[\left[\neg p_{1} \rightarrow \square\left[\left(\neg p_{1} \rightarrow \operatorname{UP}\left(t_{j}\right)\right) \wedge\left(p_{1} \rightarrow \operatorname{RIGHT}\left(t_{j}\right)\right)\right]\right]\right. \\
& \wedge \bigwedge_{i=1}^{n-1}\left[( \neg p _ { i + 1 } \wedge \bigwedge _ { k = 1 } ^ { i } p _ { k } ) \rightarrow \square \left[\left(\neg p_{i+1} \rightarrow \operatorname{UP}\left(t_{j}\right)\right)\right.\right. \\
& \left.\left.\left.\wedge\left(p_{i+1} \rightarrow \operatorname{RIGHT}\left(t_{j}\right)\right)\right]\right] \wedge\left[\left(\bigwedge_{i=1}^{n} p_{i} \wedge \bigvee_{i=1}^{n} \neg q_{i}\right) \rightarrow \square \operatorname{UP}\left(t_{j}\right)\right]\right], \quad 0 \leq j \leq m
\end{aligned}
$$

Figure 7: Formulas that encode a grid and a tiling over serial frames.

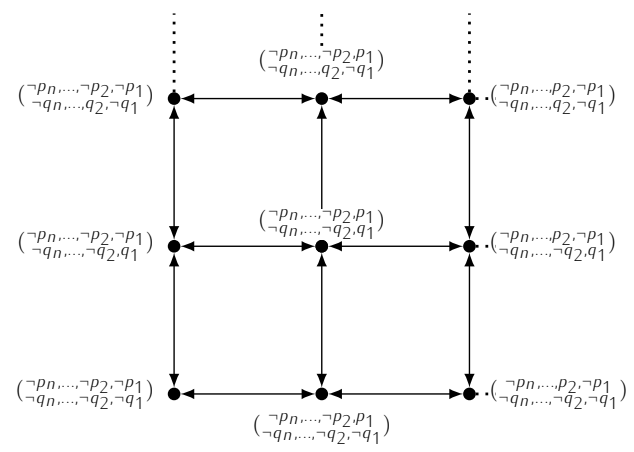

Tiling $^{\text {Sym }}$ encodes a suitable tiling for this model (see Fig. 6). Even though the formula-scheme Til $_{j}^{\text {Sym }}$ looks appallingly expanded, it is still computable in polynomial time in $n$ and $m$ (in fact, for the sake of transparency, it could be divided into 15 separate implications for each kind of point, but it would not affect the complexity of the whole Tiling ${ }^{\text {Sym }}$ formula) as well as Grid ${ }^{\text {Sym }}$. Hence, we get the conclusion. $\dashv$

THEOREM 5.21. Modal logic with global counting operators defined over the class of serial frames $\left(\mathcal{M}_{\mathrm{D}}\left(\mathrm{E}_{n}\right)\right)$ is NEXPTIME-complete.

Proof. For the upper bound, we employ Theorem 5.16. We note that a reduct of a serial model is also serial. It follows from the construction of a reduced model - every world that had a successor in the initial model will also have a successor in the reduct. 
Establishing the lower bound is relatively easy in the case of serial frames. We can see that a model forced by Grid is almost serial. This image is ruined solely by the "end" point $\left(p_{n}, \ldots, p_{1}, q_{n}, \ldots, q_{1}\right)$. It therefore suffices to add a reflexive arrow to this point, which Grid ${ }^{\text {Ser }}$ does as we can see in the picture below

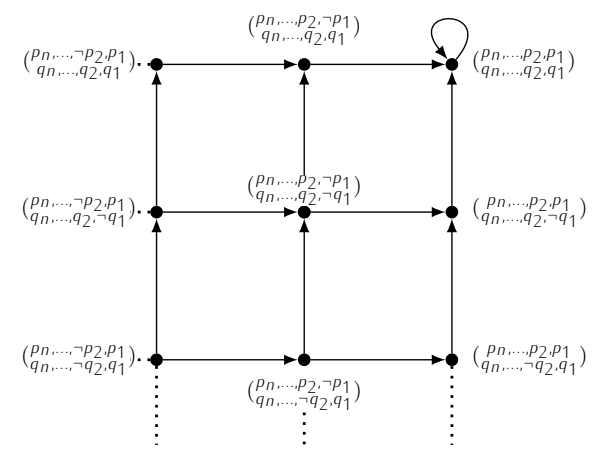

Tiling $^{\text {Ser }}$ deals with tiling for such a slightly modified model (see Fig. 7).

Grid $^{\text {Ser }} \wedge$ Tiling $^{\text {Ser }}$ is still computable in polynomial time in $n$ and $m$. Hence, the conclusion.

THEOREM 5.22. Modal logic with global counting operators defined over the class of transitive frames $\left(\mathcal{M}_{K 4}\left(E_{n}\right)\right)$ is NEXPTIME-complete.

Proof. For the upper bound we appropriately take advantage of Theorem 5.16. Let $\mathfrak{M}$ be a transitive model for a formula $\varphi$. We construct a reduced, finite model in two steps. First, we build a reduct $\mathfrak{M}^{\prime}$ in exactly the same way as in Theorem 5.16. After obtaining $\mathfrak{M}$ we take the transitive closure $R^{\prime+}$ of the relation $R^{\prime}$, which is defined as follows: $R^{\prime+}=R^{\prime} \cup\left\{\langle w, v\rangle \mid\right.$ there exists $z \in W^{\prime}$ such that $\langle w, z\rangle \in R^{\prime}$ and $\left.\langle z, v\rangle \in R^{\prime}\right\}$. By construction, $\mathfrak{M}^{\prime+}=\left\langle W^{\prime}, R^{\prime+}, V^{\prime}\right\rangle$ is a transitive structure. By Theorem $5.16 \mathfrak{M}^{\prime}$ satisfies $\varphi$ but is not necessarily a transitive model. The only thing to be shown is that taking $R^{\prime+}$ instead of $R^{\prime}$ does not violate the conditions a model has to fulfil. First, note that $R^{\prime+}$ does not introduce any pairs $\langle s, t\rangle$ such that $S$ is not already a predecessor in $R^{\prime}$. In other words, no $R^{\prime+}$-arrow goes out of $w \in W^{\prime}$ in $\mathfrak{M}^{\prime+}$ if there were no $R^{\prime}$-arrow going out of $w$ in $\mathfrak{M}^{\prime}$. The key point of this observation is this: no global "at-most" conditions can be violated by taking the transitive closure of $R^{\prime}$. For 
instance, if $\mathrm{E}_{<n} \diamond T \in \operatorname{Sub}(\varphi)$ (a formula expressing that there are at most $n-1$ worlds having $R^{\prime}$-successors) holds in $\mathfrak{M}^{\prime}$, then it will also hold in $\mathfrak{M}^{\prime+}$ since $R^{\prime+}$ introduces no additional predecessors ${ }^{7}$. Now for the main part. Assume that $\langle w, z\rangle,\langle z, v\rangle \in R^{\prime}$ but $R^{\prime+} \ni\langle w, v\rangle \notin R^{\prime}$. We need to show that no modal conflict arises between $w$ and $v$. The only situation violating the consistency of $\mathfrak{M}^{\prime+}$ that might occur is if $\mathfrak{M}^{+}, w \Vdash \neg \nabla \varphi$ and $\mathfrak{M}^{+}, v \Vdash \varphi$ for some $\nabla \varphi \in \operatorname{Sub}(\varphi)$. Suppose that this is the case. Since $\nabla \varphi \in \operatorname{Sub}(\varphi), \mathfrak{M}^{\prime+}, z \Vdash \nabla \varphi$. It follows from the fact that $\langle z, v\rangle \in R^{\prime}$, so $\mathfrak{M}^{\prime+}, z \Vdash \neg \nabla \varphi$ would contradict the consistency of $\mathfrak{M}^{\prime}$. By construction of $\mathfrak{M}^{\prime}$, there exists $z^{\prime} \in W$ (the universe of the initial model) such that $z^{\prime} \rightsquigarrow \operatorname{Sub}(\varphi) z$ and $\left\langle w, z^{\prime}\right\rangle \in R$. What follows, $\mathfrak{M}, z^{\prime} \Vdash \nabla \varphi$. Consequently, there exists $x \in W$ such that $\left\langle z^{\prime}, x\right\rangle \in R$ and $\mathfrak{M}, x \Vdash \varphi$. But by transitivity of $R$ we get $\langle w, x\rangle \in R$ and by the consistency of $\mathfrak{M} \mathfrak{M}, w \Vdash \diamond \varphi$ is the case. By construction of $\mathfrak{M}^{\prime+}$ that contradicts the assumption that $\mathfrak{M}^{\prime+}, w \Vdash \neg \diamond \varphi$. Thus, $\mathfrak{M}^{\prime+}$ is a finite and transitive model for $\varphi$.

For the lower bound, we establish the encoding of an exponentialsize tiling problem along the lines of the proof of NEXPTIME-completeness for graded modal logic defined over transitive frames, presented in [61]. However, we simplify the techniques employed by Kazakov and Pratt-Hartmann to obtain a grid-shaped model, and remove elements which are, in our opinion, superfluous.

First, we need to observe that due to the transitive character of the accessibility relation, the construction of a grid in a manner similar to (the one) in the proof of Lemma 5.18 immediately turns out infeasible. It is because after taking the transitive closure of the grid formed by the formulas shown in Fig. 3, each point with a pair of coordinates $(i, j)$ is linked to every point whose coordinates $(k, l)$ satisfy the following condition:

$$
(k>i \wedge l \geq j) \vee(k \geq i \wedge l>j) .
$$

The picture below visualises a fragment of a model obtained as a consequence. We can easily notice that it no longer preserves properties of a grid.

7 Also worth noting is that $E_{>n}$ and $E_{<n}$ operators do not directly detect the number of successors of a world. They are able to achieve it in a roundabout way by encoding nominals. This, however, is not a threat to the construction of a transitive reduct of $\mathfrak{M}$. For a detailed discussion see [6]. 
Tree $^{\text {Tran }}$ formulas:

$$
\begin{aligned}
\mathrm{IP}^{\operatorname{Tran}}:= & \mathrm{E}_{=1} v_{0} \\
\operatorname{Inc}_{i}^{\operatorname{Tran}}:= & v_{i} \rightarrow\left[\diamond\left(v_{i+1} \wedge \neg p_{i+1} \wedge \neg q_{i+1}\right) \wedge \diamond\left(v_{i+1} \wedge \neg p_{i+1} \wedge q_{i+1}\right)\right. \\
& \wedge \diamond\left(v_{i+1} \wedge p_{i+1} \wedge \neg q_{i+1}\right) \wedge \diamond\left(v_{i+1} \wedge p_{i+1} \wedge q_{i+1}\right) \\
& \left.\wedge \wedge_{k=1}^{i}\left(\mathrm{UNC}\left(p_{k}\right) \wedge \mathrm{UNC}\left(q_{i}\right)\right)\right], \quad 0 \leq i<n \\
\mathrm{Un}_{p}^{\text {Tran }}:= & \bigwedge_{0 \leq i<j<n}\left(v_{i} \rightarrow \neg v_{j}\right) \\
\operatorname{Num}_{i}^{\text {Tran }}:= & \mathrm{E}_{=2^{2 i} v_{i}, \quad 1 \leq i \leq n}
\end{aligned}
$$

Figure 8: Formulas that force a tree, the bottom "layer" of which will be arranged as a grid.

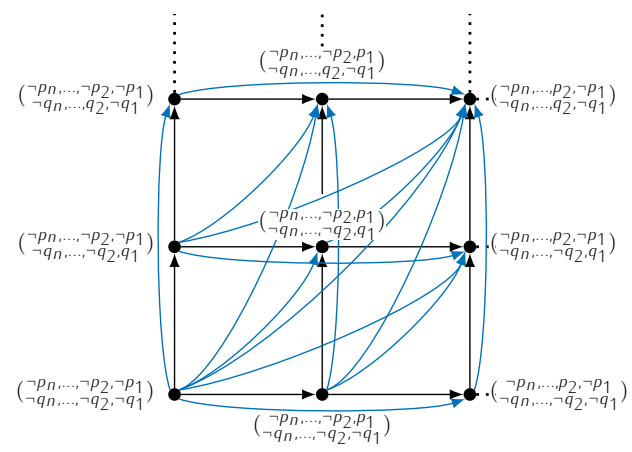

Keeping in mind that the previously employed way of linking-up coordinated points into a grid fails in the case of transitive frames, we need to split up this process into two separate parts. We first introduce $2^{2 n}$ distinct, coordinated points and afterwards we make an attempt to arrange links between them, so that the whole would form some kind of a grid.

We again pick $2 n$ propositional variables $p_{1}, \ldots p_{n}, q_{1}, \ldots, q_{n}$ which constitute our coordinates. Additionally, we take $n+1$ propositional variables $v_{0}, \ldots, v_{n}$, distinct from the former, which are used to determine, in a certain way, the location of a particular state in a model.

We begin with introducing the initial point, which is done by the formula IPTran in Fig. 8. Subsequently, we start to raise a construction that is a transitive, quaternary tree. As it is roughly visualised in the 
picture below, the tree consists of consecutive layers, each of which has its index depending on its placement relative to the initial point:

$(0,0)$

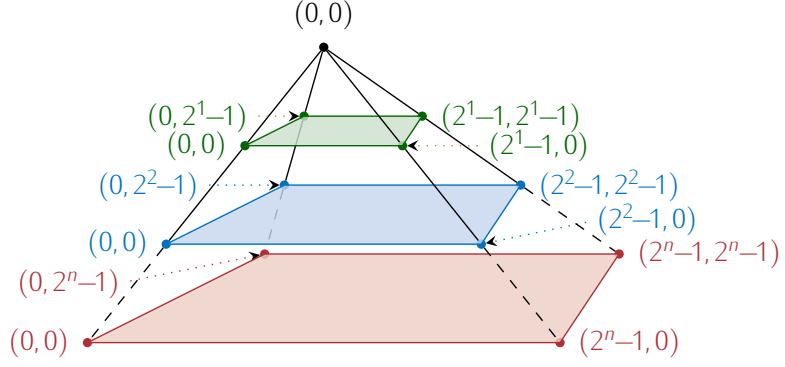

And so, the initial point, which is also the root of the tree, has an index 0 and satisfies $v_{0}$. The layer of direct successors of the initial point has an index 1 and satisfies $v_{1}$ :

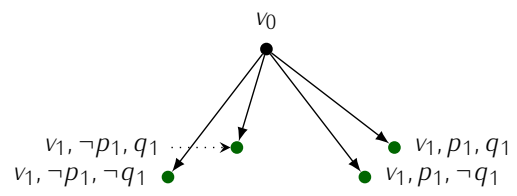

It works in exactly the same way for subsequent layers. The formula $U \mathrm{n}_{\mathrm{p}}^{\text {Tran }}$ asserts that for every $i \in\{1, \ldots, n\}$ the formula $v_{i}$ is uniquely associated with the $i$-th layer. As indicated by the formula-scheme Inc $_{i}^{\text {Tran }}$, every point situated in a layer with an index $i \in\{0, \ldots, n-1\}$ has four direct successors ${ }^{8}$. In the following image, a fragment of a tree separated in this way is shown. However, to get a full picture of how this tree-shaped fragment looks like, we need to take the transitive closure of the presented part.

8 To be more precise, every point has at least four direct successors. Formula-schemes $\operatorname{Inc}_{i}^{\text {Tran }}$ do not exclude the possibility that some of these points have more than four successors. However, only four of them are relevant for our construction. The tool that serves to distinguishing relevant successors from irrelevant ones is the set of propositional variables $v_{0}, \ldots, v_{n}$. The formula-scheme $\operatorname{Inc}_{i}^{T r a n}$ assures that in every relevant successor, located in an $i$-th layer, a formula $v_{i}$ is satisfied, and the formula scheme Num $_{i}^{\text {Tran }}$ secures that $v_{i}$ is only satisfied by relevant successors. Thus, we can safely focus on the states that satisfy a formula $v_{i}$ for some $i \in\{0, \ldots, n\}$. 


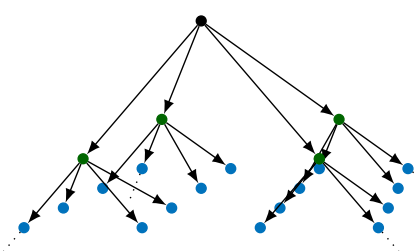

The formulas $\operatorname{Inc}_{i}^{\text {Tran }}$ act twofold. First, for each state they increment an index of its successors, indicating that the latter are located in a consecutive layer. Second, they introduce an additional digit (formulas $p_{i}$ and $q_{i}$ for the $i$-th layer) to each coordinate, thereby doubling its range. The following example explains how this works in practice.

EXAMPLE 5.1. We consider a point with coordinates $(001,110)$. Since either of its coordinates consist of 3 digits, it indicates that it is located in the 3rd layer, so it satisfies the formula $v_{3}$. It has four direct successors with 4-digit coordinates that preserve values of the first 3 digits (counting from the right). Every direct successor satisfies one of the four possible pairs of additional digits.

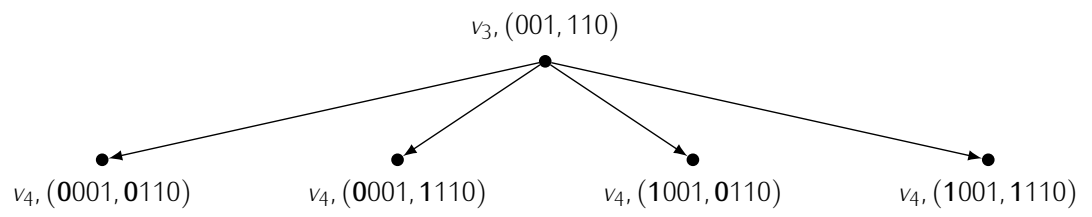

Obviously, each of the formulas $p_{1}, \ldots, p_{n}, q_{1}, \ldots, q_{n}$ has a particular logical value at every state of our model. However, this does not mean that each of these formulas is always a part of a coordinate. Formulas $v_{0}, \ldots, v_{n}$ point out which formulas should be read off as constituting a coordinate. For example, if $v_{i}$ is satisfied by a point, only the formulas $p_{1}, \ldots, p_{i}, q_{1}, \ldots, q_{i}$ form coordinates at this point, the rest being irrelevant.

Since we want to encode the exponential tiling problem, in which $k \times k, k=2^{n}$ is the size of the grid, the subject of our interest is the bottom, $n$-th layer ${ }^{9}$. It consists of $2^{2 n}$ distinct, coordinated points.

9 However, besides the 0 -th layer, each layer up to the $n$-th could be utilised to encode the exponential tiling problem. 
It can be easily verified by simple induction that for every pair of coordinates $(i, j) \in 2^{n}-1 \times 2^{n}-1$ there exists a point with the pair of coordinates $(i, j)$ in the $n$-th layer. The remaining part of our work is to appropriately link up these points, so that they form a grid.

As announced beforehand, we cannot directly connect the adjacent (with respect to coordinates) points, for due to the transitivity of the accessibility relation it would immediately ruin our grid. We therefore introduce another two distinct propositional variables: $n_{r}$ (for the right neighbour) and $n_{u}$ (for the up neighbour). They will label points that are located between, respectively, two right-adjacent (with respect to coordinates) points and two up-adjacent (with respect to coordinates) points. The accessibility arrows departing from $v_{n}$ points are directed to $n_{r}$ and $n_{u}$ points rather than to adjacent $v_{n}$ points, which allows us to avoid destructive effects of the transitivity of the accessibility relation. The formulas $U \mathrm{n}_{\mathrm{r}}^{\mathrm{Tran}}$ and $\mathrm{Un}_{\mathrm{u}}^{\text {Tran }}$ guarantee that $n_{r}$ and $n_{u}$ points are distinct from every point that has been thus far introduced in our construction. The formulas Num $\mathrm{r}_{r}^{\text {Tran }}$ and Num $\mathrm{N}_{\mathrm{u}}^{\text {Tran }}$ appropriately limit the number of $n_{r}$ and $n_{u}$ points. $n_{r}$ points "inherit" coordinates from their right-neighbours, whereas $n_{u}$ points take coordinates after their up-neighbours. It is assured by the formulas $\operatorname{Inh}_{i, j}^{\text {Tran }}$ displayed in Fig. 9. Again, different Inh formulas parallel different kinds of points, which, depending on coordinates, can have from two to four $n$ neighbours. In the following picture, the arrangement of the bottom layer of the tree is presented. Hollow dots represent $n_{r}$ and $n_{u}$ points, whereas full dots stand for points constituting a proper grid. It is easy to notice that $n_{r}$ and $n_{u}$ are "sink" points which allows to preserve adjacency relations despite the transitivity of the accessibility relation. 


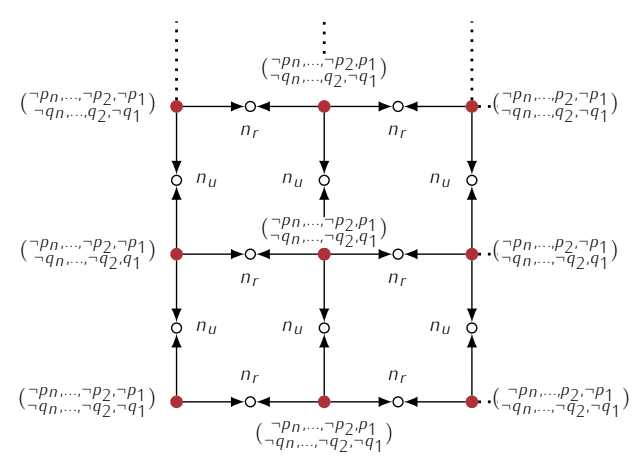

The only missing element of the puzzle is an appropriate encoding of a tiling. This is taken care of by the formulas in Fig. 9 (Note that the transitivity of the accessibility relation does not interfere with this setting). In Til Tran we can see that $n_{r}$-worlds and $n_{u}$-worlds were introduced to "store" the information about a tile type used to cover its, respectively, right- and up-neighbour. In this way $v_{n}$ points have the indirect access to points located adjacently (with respect to coordinates).

To complete the proof, we need to gather up all that has been established so far. The following formulas encode, respectively, a tree, a grid, and a tiling:

$$
\begin{aligned}
& \text { Tree }^{\text {Tran }}:=\mathrm{IP}^{\text {Tran }} \wedge \mathrm{A}\left(\mathrm{Un}_{p}^{\text {Tran }} \wedge \bigwedge_{i=0}^{n} \mathrm{Inc}_{i}^{\text {Tran }} \wedge \bigwedge_{i=1}^{n} \mathrm{Num}_{i}^{\text {Tran }}\right) \\
& \text { Grid }^{\text {Tran }}:=\mathrm{A}\left(\mathrm{Un}_{r}^{\text {Tran }} \wedge \mathrm{Un}_{u}^{\text {Tran }} \wedge \bigwedge_{i, j=0}^{n} \operatorname{lnh}_{i, j}^{\text {Tran }} \wedge \operatorname{lnh}_{0 *, 0 *}^{\text {Tran }}\right. \\
& \left.\wedge \bigwedge_{i=0}^{n} \operatorname{lnh}_{i, 0 *}^{\text {Tran }} \wedge \bigwedge_{j=0}^{n} \operatorname{lnh}_{0 *, j}^{\text {Tran }}\right) \wedge \operatorname{Num}_{r}^{\text {Tran }} \wedge \operatorname{Num}_{u}^{\text {Tran }} \\
& \text { Tiling }^{\text {Tran }}:=\mathrm{A}\left(\mathrm{Cov}^{\text {Tran }} \wedge \mathrm{Un}^{\text {Tran }} \wedge \mathrm{IPC}^{\text {Tran }} \wedge \bigwedge_{j=0}^{m} \operatorname{Til}_{j}^{\text {Tran }}\right)
\end{aligned}
$$

To verify that $\varphi:=$ Tree $^{\text {Tran }} \wedge$ Grid $^{\text {Tran }} \wedge$ Tiling $^{\text {Tran }}$ encodes a suitable tiling, we need to proceed in the analogous way to the proof of Lemma 5.18, and define an appropriate translation function. Checking that $\varphi$ is satisfiable on a transitive frame is a matter of routine. Note that all formulas constituting $\varphi$ have the size polynomial in $n$. The only formulas that might raise doubts are Num ${ }^{\text {Tran }}$. However, due to the binary coding of numerical subscripts, the actual size of the subscript in a formula $\operatorname{Num}_{i}^{\text {Tran }}=\mathrm{E}_{=22} v_{i}$ is $2 i$ for $i \in\{1, \ldots, n\}$ and 1 for $i=0$. What follows, the size of the numerical subscripts in formulas Num ${ }_{r}^{\text {Tran }}$ 


\section{Grid $^{\text {Tran }}$ formulas:}

$$
\begin{aligned}
& \operatorname{Inh}_{0 *, 0 *}^{\operatorname{Tran}}:=\left(\bigwedge_{i=1}^{n} \neg p_{i} \wedge \bigwedge_{j=1}^{n} \neg q_{j}\right) \rightarrow\left[\diamond n_{r} \wedge \diamond \neg n_{u} \wedge \bigwedge_{i=2}^{n} \operatorname{UNC}\left(p_{i}\right)\right. \\
& \wedge \bigwedge_{j=2}^{n} \operatorname{UNC}\left(q_{j}\right) \wedge \square\left[\left(n_{r} \rightarrow\left(p_{1} \wedge \neg q_{1}\right)\right) \wedge\left(\left(n_{u} \rightarrow\left(\neg p_{1} \wedge q_{1}\right)\right)\right]\right] \\
& \operatorname{Inh}_{0 *, 0}^{\operatorname{Tran}}:=\left(\bigwedge_{i=1}^{n} \neg p_{1} \wedge q_{j+1} \wedge \bigwedge_{k=1}^{j} \neg q_{k}\right) \rightarrow\left[\diamond n_{r} \wedge \diamond\left(n_{u} \wedge q_{1}\right)\right. \\
& \wedge \diamond\left(n_{u} \wedge \neg q_{1}\right) \wedge \bigwedge_{i=2}^{n} \operatorname{UNC}\left(p_{i}\right) \wedge \bigwedge_{j=2}^{n}\left(q_{j}\right) \\
& \left.\wedge \square\left[\left(n_{r} \rightarrow\left(p_{1} \wedge \neg q_{1}\right)\right) \wedge\left(n_{u} \rightarrow \neg p_{1}\right)\right]\right], \quad 1 \leq j<n \\
& \operatorname{Inh}_{0,0}^{\text {Tran }}:=\left(p_{i+i} \wedge \bigwedge_{k=1}^{i} \neg p_{k} \wedge q_{j+1} \wedge \wedge_{l=1}^{j}\right) \rightarrow\left[\diamond\left(n_{r} \wedge \neg p_{1}\right) \wedge \diamond\left(n_{r} \wedge p_{1}\right)\right. \\
& \wedge \diamond\left(n_{u} \wedge \neg q_{1}\right) \wedge \diamond\left(n_{u} \wedge q_{1}\right) \wedge \bigwedge_{i=2}^{n} \operatorname{UNC}\left(p_{i}\right) \wedge \bigwedge_{j=2}^{n} \operatorname{UNC}\left(q_{j}\right) \\
& \left.\wedge \square\left[\left(n_{r} \rightarrow \neg q_{1}\right) \wedge\left(n_{u} \rightarrow \neg p_{1}\right)\right]\right], \quad 0<i, j<n \\
& \operatorname{Inh}_{0 *, j}^{\operatorname{Tran}}:=\left(\bigwedge_{i=1}^{n} \neg p_{i} \wedge \neg q_{j+1} \wedge \bigwedge_{k=1}^{j} q_{k}\right) \rightarrow\left[\diamond n_{r} \wedge \diamond\left(n_{u} \wedge \neg q_{j+1}\right)\right. \\
& \wedge \diamond\left(n_{u} \wedge q_{j+1}\right) \wedge \bigwedge_{i=2}^{n} \operatorname{UNC}\left(p_{i}\right) \wedge \bigwedge_{k=j+2}^{n} \operatorname{UNC}\left(q_{k}\right) \\
& \wedge \square\left[\left(n_{r} \rightarrow\left(p_{1} \wedge \neg q_{j+1} \bigwedge_{k=1}^{j} q_{k}\right)\right)\right. \\
& \wedge\left(\left(n_{u} \wedge \neg q_{j+1}\right) \rightarrow\left(\neg p_{1} \wedge \wedge_{k=1}^{j} q_{k}\right)\right) \\
& \left.\left.\wedge\left(\left(n_{u} \wedge \neg q_{j+1}\right) \rightarrow\left(\neg p_{1} \wedge \wedge_{k=1}^{j} \neg q_{k}\right)\right)\right]\right], \quad 0<j<n \\
& \operatorname{Inh}_{0, j}^{\text {Tran }}:=\left(p_{i+1} \wedge \bigwedge_{k=1}^{i} \neg p_{k} \wedge \neg q_{j+1} \wedge \bigwedge_{l=1}^{j} q_{l}\right) \rightarrow\left[\diamond\left(n_{r} \wedge \neg p_{1}\right) \wedge \diamond\left(n_{r} \wedge p_{1}\right)\right. \\
& \wedge \diamond\left(n_{u} \wedge \neg q_{j+1}\right) \wedge \diamond\left(n_{u} \wedge q_{j+1}\right) \wedge \bigwedge_{i=2}^{n} \operatorname{UNC}\left(p_{i}\right) \wedge \bigwedge_{k=j+2}^{n} \operatorname{UNC}\left(q_{k}\right) \\
& \wedge \square\left[\left(n_{r} \rightarrow\left(\neg q_{j+1} \wedge \bigwedge_{k=1}^{j} q_{k}\right) \wedge\left(\left(n_{u} \wedge \neg q_{j+1}\right) \rightarrow\left(\neg p_{1} \wedge \bigwedge_{k=1}^{j} q_{k}\right)\right)\right.\right. \\
& \left.\left.\wedge\left(\left(n_{u} \wedge q_{j+1}\right) \rightarrow\left(\neg p_{1} \wedge \wedge_{k=1}^{j} \neg q_{k}\right)\right)\right]\right], \quad 1 \leq j<n \\
& \operatorname{Inh}_{i, j}^{\text {Tran }}:=\left(\neg p_{i+1} \wedge \bigwedge_{k=1}^{i} p_{k} \wedge \neg q_{j+1} \wedge \bigwedge_{l=1}^{j} q_{j}\right) \rightarrow\left[\diamond\left(n_{r} \wedge \neg p_{i+1}\right) \wedge \diamond\left(n_{r} \wedge p_{i+1}\right)\right. \\
& \wedge \diamond\left(n_{u} \wedge \neg q_{j+1}\right) \wedge \diamond\left(n_{u} \wedge q_{j+1}\right) \wedge \bigwedge_{k=i+2}^{n} \operatorname{UNC}\left(p_{k}\right) \wedge \bigwedge_{l=j+2}^{n} \operatorname{UNC}\left(q_{l}\right) \\
& \wedge \square\left[\left(\left(n_{r} \wedge \neg p_{i+1}\right) \rightarrow\left(\bigwedge_{k=2}^{i} p_{k} \wedge \neg q_{j+1} \bigwedge_{l=1}^{j} q_{l}\right)\right)\right. \\
& \wedge\left(\left(n_{r} \wedge p_{i+1}\right) \rightarrow\left(\bigwedge_{k=2}^{i} \neg p_{k} \wedge \neg q_{j+1} \wedge \bigwedge_{l=1}^{j} q_{l}\right)\right) \\
& \wedge\left(\left(n_{u} \wedge \neg q_{j+1}\right) \rightarrow\left(\neg p_{i+1} \wedge \bigwedge_{k=1}^{i} p_{k} \wedge \bigwedge_{l=1}^{j} q_{l}\right)\right) \\
& \left.\left.\wedge\left(\left(n_{u} \wedge q_{j+1}\right) \rightarrow\left(\neg p_{i+1} \wedge \bigwedge_{k=1}^{i} p_{k} \wedge \bigwedge_{l=1}^{j} \neg q_{l}\right)\right)\right]\right], \quad 1 \leq i, j<n
\end{aligned}
$$

Figure 9: Formulas that link up points from the $n$-th, bottom layer of the tree into a grid, and encode a tiling. 


$$
\begin{aligned}
& \operatorname{Inh}_{n, 0 *}^{\operatorname{Tran}}:=\left(\bigwedge_{i=1}^{n} p_{i} \wedge \bigwedge_{j=1}^{n} \neg q_{j}\right) \rightarrow\left[\diamond n_{r} \wedge \diamond \neg n_{u} \wedge \bigwedge_{i=2}^{n} \operatorname{UNC}\left(p_{i}\right) \wedge \bigwedge_{j=2}^{n} \operatorname{UNC}\left(q_{j}\right)\right. \\
& \left.\wedge \square\left[\left(n_{r} \rightarrow\left(\neg p_{1} \wedge \neg q_{1}\right)\right) \wedge\left(n_{u} \rightarrow\left(p_{1} \wedge q_{1}\right)\right)\right]\right] \\
& \operatorname{Inh}_{n, 0}^{\text {Tran }}:=\left(\bigwedge_{i=1}^{n} p_{i} \wedge q_{j+1} \wedge \bigwedge_{k=1}^{j} \neg q_{k}\right) \rightarrow\left[\diamond n_{r} \wedge \diamond\left(n_{u} \wedge \neg q_{1}\right)\right. \\
& \wedge \diamond\left(n_{u} \wedge q_{1}\right) \wedge \bigwedge_{i=2}^{n} \operatorname{UNC}\left(p_{i}\right) \wedge \bigwedge_{j=2}^{n} \operatorname{UNC}\left(q_{j}\right) \\
& \left.\wedge \square\left[\left(n_{r} \rightarrow\left(\neg p_{1} \wedge \neg q_{1}\right)\right) \wedge\left(n_{u} \rightarrow p_{1}\right)\right]\right], \quad 1 \leq i, j<n \\
& \operatorname{Inh}_{n, j}^{\text {Tran }}:=\left(\bigwedge_{i=1}^{n} p_{i} \wedge \neg q_{j+1} \wedge \bigwedge_{k=1}^{j} q_{k}\right) \rightarrow\left[\diamond n_{r} \wedge \diamond\left(n_{u} \wedge \neg q_{j+1}\right)\right. \\
& \wedge \diamond\left(n_{u} \wedge q_{j+1}\right) \wedge \bigwedge_{i=2}^{n} \operatorname{UNC}\left(p_{i}\right) \wedge \bigwedge_{k=j+2}^{n} \operatorname{UNC}\left(q_{k}\right) \\
& \wedge \square\left[\left(n_{r} \rightarrow\left(\neg p_{1} \wedge \neg q_{j+1} \wedge \wedge_{k=1}^{j} q_{k}\right)\right)\right. \\
& \wedge\left(\left(n_{u} \wedge \neg q_{j+1}\right) \rightarrow\left(p_{1} \wedge \wedge_{k=1}^{j} q_{k}\right)\right) \\
& \left.\left.\wedge\left(\left(n_{u} \wedge q_{j+1}\right) \rightarrow\left(p_{1} \wedge \wedge_{k=1}^{j} \neg q_{k}\right)\right)\right]\right], \quad 1 \leq j<n \\
& \operatorname{Inh}_{n, n}^{\operatorname{Tran}}:=\left(\bigwedge_{i=1}^{n} p_{i} \wedge \bigwedge_{j=1}^{n} q_{j}\right) \rightarrow\left[\diamond n_{r} \wedge \diamond_{u} \wedge \bigwedge_{i=2}^{n} \operatorname{UNC}\left(p_{i}\right) \wedge \bigwedge_{j=2}^{n} \operatorname{UNC}\left(q_{j}\right)\right. \\
& \left.\wedge \square\left[\left(n_{r} \rightarrow\left(\neg p_{1} \wedge q_{1}\right)\right) \wedge\left(n_{u} \rightarrow\left(p_{1} \wedge \neg q_{1}\right)\right)\right]\right]
\end{aligned}
$$

Tiling Tran formuls:

$$
\begin{aligned}
\operatorname{Num}_{r}^{\operatorname{Tran}} & :=\mathrm{E}_{=\left(2^{2 n}-2^{n}\right)^{n_{r}}} \\
\operatorname{Num}_{u}^{\operatorname{Tran}} & :=\mathrm{E}_{=\left(2^{2 n}-2^{n}\right)^{n_{u}}} \\
\mathrm{Un}_{r}^{\operatorname{Tran}} & :=n_{r} \rightarrow\left(\neg n_{u} \wedge \bigwedge_{i=0}^{n} \neg v_{n}\right) \\
\mathrm{Un}_{u}^{\operatorname{Tran}} & :=n_{u} \rightarrow\left(\neg n_{r} \wedge \bigwedge_{i=0}^{n} \neg v_{n}\right) \\
\operatorname{Cov}^{\text {Tran }} & :=v_{n} \rightarrow \bigvee_{i=0}^{m} t_{i} \\
\mathrm{Un}^{\text {Tran }} & :=v_{n} \rightarrow\left[\bigwedge_{0 \leq i<j \leq m}\left(t_{i} \rightarrow \neg t_{j}\right)\right] \\
\operatorname{IPC}^{\operatorname{Tran}} & :=\left(v_{n} \wedge \bigwedge_{i=1}^{n} \neg p_{i} \wedge \bigwedge_{j=1}^{n} \neg q_{j}\right) \rightarrow t_{0}
\end{aligned}
$$

Continued Figure 9: Formulas that link up points from the $n$-th, bottom layer of the tree into a grid, and encode a tiling. 


$$
\begin{aligned}
& \mathrm{Til}_{j}^{\text {Tran }}:=\left(v_{n} \wedge t_{j}\right) \rightarrow\left[( \bigwedge _ { i = 1 } ^ { n } \neg p _ { i } \wedge \bigwedge _ { j = 1 } ^ { n } \neg q _ { j } ) \rightarrow \square \left[\left(\left(n_{u}\right) \rightarrow \operatorname{UP}\left(t_{j}\right)\right.\right.\right. \\
& \left.\left.\wedge\left(n_{r} \rightarrow \operatorname{RIGHT}\left(t_{j}\right)\right)\right]\right] \\
& \wedge \bigwedge_{j=1}^{n-1}\left[( \bigwedge _ { i = 1 } ^ { n } \neg p _ { i } \wedge q _ { j + 1 } \wedge \bigwedge _ { k = 1 } ^ { j } \neg q _ { k } ) \rightarrow \square \left[\left(\left(n_{u} \wedge \neg q_{1}\right) \rightarrow t_{j}\right)\right.\right. \\
& \left.\left.\left.\wedge\left(n_{u} \wedge q_{1}\right) \rightarrow \operatorname{UP}\left(t_{j}\right)\right) \wedge\left(n_{r} \rightarrow \operatorname{RIGHT}\left(t_{j}\right)\right)\right]\right] \\
& \wedge \bigwedge_{j=1}^{n-1}\left[\left(\bigwedge_{i=1}^{n} \neg p_{i} \wedge \neg q_{j+1} \bigwedge_{k=1}^{n} q_{k}\right) \rightarrow \square\left[\left(n_{u} \wedge \neg q_{j+1}\right) \rightarrow t_{j}\right)\right. \\
& \left.\wedge\left(\left(n_{u} \wedge q_{j+1}\right) \rightarrow \operatorname{UP}\left(t_{j}\right) \wedge\left(n_{r} \rightarrow \operatorname{RIGHT}\left(t_{j}\right)\right)\right]\right] \\
& \wedge\left[\left(\bigwedge_{i=1}^{n} \neg p_{i} \wedge \bigwedge_{j=1}^{n} q_{j}\right) \rightarrow \square\left[\left(n_{u} \rightarrow \mathrm{t}_{j}\right) \wedge\left(n_{r} \rightarrow \operatorname{RIGHT}\left(t_{j}\right)\right)\right]\right] \\
& \wedge \bigwedge_{i=1}^{n-1}\left[( p _ { i + 1 } \wedge \bigwedge _ { k = 1 } ^ { i } \neg p _ { k } \wedge \bigwedge _ { j = 1 } ^ { n } \neg q _ { j } ) \rightarrow \square \left[\left(n_{u} \rightarrow \operatorname{UP}\left(t_{j}\right)\right.\right.\right. \\
& \left.\left.\wedge\left(\left(n_{r} \wedge \neg p_{1}\right) \rightarrow t_{j}\right) \wedge\left(\left(n_{r} \wedge p_{1}\right) \rightarrow \operatorname{RIGHT}\left(t_{j}\right)\right)\right]\right] \\
& \wedge \bigwedge_{i, j=1}^{n-1}\left[( p _ { i + 1 } \wedge \bigwedge _ { k = 1 } ^ { i } \neg p _ { k } \wedge q _ { j + 1 } \wedge \bigwedge _ { l = 1 } ^ { j } \neg q _ { j } ) \rightarrow \square \left[\left(\left(\neg p_{1} \wedge \neg q_{1}\right) \rightarrow t_{j}\right)\right.\right. \\
& \wedge\left(q_{1} \rightarrow \operatorname{UP}\left(t_{j}\right) \wedge\left(p_{1} \rightarrow \operatorname{RIGHT}\left(t_{j}\right)\right]\right] \\
& \wedge \bigwedge_{i, j=1}^{n-1}\left[( p _ { i + 1 } \wedge \bigwedge _ { k = 1 } ^ { i } \neg p _ { i } \wedge \neg q _ { j + 1 } \wedge \bigwedge _ { l = 1 } ^ { j } q _ { l } ) \rightarrow \square \left[\left(\left(\neg p_{1} \wedge \neg q_{j+1}\right) \rightarrow t_{j}\right)\right.\right. \\
& \left.\left.\wedge\left(q_{j+1} \rightarrow \operatorname{UP}\left(t_{j}\right)\right) \wedge\left(p_{1} \rightarrow \operatorname{RIGHT}\left(t_{j}\right)\right)\right]\right] \\
& \wedge \bigwedge_{i=1}^{n-1}\left[( p _ { i + 1 } \wedge \bigwedge _ { k = 1 } ^ { i } \neg p _ { k } \wedge \bigwedge _ { j = 1 } ^ { n } q _ { j } ) \rightarrow \square \left[\left(\neg p_{1} \rightarrow t_{j}\right)\right.\right. \\
& \left.\left.\wedge\left(p_{1} \rightarrow \operatorname{RIGHT}\left(t_{j}\right)\right)\right]\right] \\
& \wedge \bigwedge_{i=1}^{n-1}\left[( \neg p _ { i + 1 } \wedge \bigwedge _ { k = 1 } ^ { i } p _ { k } \wedge \bigwedge _ { j = 1 } ^ { n } \neg q _ { j } ) \rightarrow \square \left[\left(\left(n_{r} \wedge \neg p_{i+1}\right) \rightarrow t_{j}\right)\right.\right. \\
& \left.\left.\wedge\left(n_{u} \rightarrow \operatorname{UP}\left(t_{j}\right)\right) \wedge\left(\left(n_{r} \wedge p_{i+1}\right) \rightarrow \operatorname{RIGHT}\left(t_{j}\right)\right)\right]\right] \\
& \wedge \bigwedge_{i, j=1}^{n-1}\left[( \neg p _ { i + 1 } \wedge \bigwedge _ { k = 1 } ^ { i } p _ { k } \wedge q _ { j + 1 } \wedge \bigwedge _ { l = 1 } ^ { j } \neg q _ { l } ) \rightarrow \square \left[\left(\left(\neg p_{i+1} \wedge \neg q_{1}\right) \rightarrow t_{j}\right)\right.\right. \\
& \left.\left.\wedge\left(q_{1} \rightarrow \mathrm{UP}\left(t_{j}\right)\right) \wedge\left(p_{i+1} \rightarrow \operatorname{RIGHT}\left(t_{j}\right)\right)\right]\right] \\
& \wedge \bigwedge_{i, j=1}^{n-1}\left[( \neg p _ { i + 1 } \wedge \bigwedge _ { k = 1 } ^ { i } p _ { k } \wedge \neg q _ { j + 1 } \wedge \bigwedge _ { l = 1 } ^ { j } q _ { l } ) \rightarrow \square \left[\left(\left(\neg p_{i+1} \wedge \neg q_{j+1}\right) \rightarrow t_{j}\right)\right.\right. \\
& \left.\left.\wedge\left(q_{j+1} \rightarrow \operatorname{UP}\left(t_{j}\right)\right) \wedge\left(p_{i+1} \rightarrow \operatorname{RIGHT}\left(t_{j}\right)\right)\right]\right] \\
& \wedge \bigwedge_{i=1}^{n-1}\left[( \neg p _ { i + 1 } \wedge \bigwedge _ { k = 1 } ^ { i } p _ { k } \wedge \bigwedge _ { j = 1 } ^ { n } q _ { j } ) \rightarrow \square \left[\left(\neg p_{i+1} \rightarrow t_{j}\right)\right.\right. \\
& \left.\left.\wedge\left(p_{i+1} \rightarrow \operatorname{RIGHT}\left(t_{j}\right)\right)\right]\right] \\
& \wedge\left[\left(\bigwedge_{i=1}^{n} p_{k} \wedge \bigwedge_{j=1}^{n} q_{j}\right) \rightarrow \square\left[\left(n_{r} \rightarrow t_{j}\right) \wedge\left(n_{u} \rightarrow \mathrm{UP}\left(t_{j}\right)\right)\right]\right] \\
& \wedge \bigwedge_{j=1}^{n-1}\left[\left(\bigwedge_{i=1}^{n} p_{k} \wedge q_{j+1} \wedge \bigwedge_{l=1}^{j} \neg q_{l}\right) \rightarrow \square\left[\left(\neg q_{1} \rightarrow t_{j}\right) \wedge\left(q_{1} \rightarrow \mathrm{UP}\left(t_{j}\right)\right)\right]\right] \\
& \wedge \bigwedge_{j=1}^{n-1}\left[( \bigwedge _ { i = 1 } ^ { n } p _ { k } \wedge \neg q _ { j + 1 } \wedge \bigwedge _ { k = 1 } ^ { j } q _ { k } ) \rightarrow \square \left[\left(\neg q_{j+1} \rightarrow t_{j}\right)\right.\right. \\
& \left.\left.\wedge\left(q_{j+1} \rightarrow \mathrm{UP}\left(t_{j}\right)\right)\right]\right] \\
& \wedge\left[\left(\bigwedge_{i=1}^{n} p_{k} \wedge \bigwedge_{j=1}^{n} q_{j}\right) \rightarrow \square t_{j}\right], \quad 0 \leq j \leq m
\end{aligned}
$$

Continued Figure 9: Formulas that link up points from the $n$-th, bottom layer of the tree into a grid, and encode a tiling. 
and $\mathrm{Num}_{u}^{\text {Tran }}$ is strictly smaller than $2 n$. Let $S$ be the total size of numerical subscripts in all Num ${ }^{\text {Tran }}$ formulas. We have:

$$
S<1+2 \cdot 2 n+\sum_{i=1}^{n} 2 i=1+4 n+n(n+1)=n^{2}+5 n+1 .
$$

We therefore obtained a polynomial bound of the size of all numerical subscripts in formulas constituting $\varphi$. This concludes the proof.

THEOREM 5.23. Modal logic with global counting operators defined over the class of Euclidean frames has the finite model property with the bounding function from Theorem 5.16.

Proof. Let $\mathfrak{M}$ be a Euclidean model for $\varphi$. We build a reduct $\mathfrak{M}^{\prime}$ in a way described in Theorem 5.16. Afterwards, the proof follows similarly to the proof of Theorem 5.22. We define $R^{\prime \circ}$ as the Euclidean closure of $R^{\prime \circ}$. We only need to show that if $\langle w, v\rangle \in R^{\prime}$ and $\langle w, z\rangle \in R^{\prime}$ but $\langle v, z\rangle \notin R^{\prime}$, then adding $\langle v, z\rangle$ to $R^{\prime \circ}$ will not violate the satisfaction conditions for modal formulas in $\operatorname{Sub}(\varphi)$. This is done analogously to the proof for transitive frames.

THEOREM 5.24. Modal logic with global counting operators defined over the class of equivalence relation frames has the finite model property with the bounding function from Theorem 5.16.

Proof. Let $\mathfrak{M}=\langle W, R, V\rangle$ be a model for a formula $\varphi$, where $R$ is an equivalence relation. We construct a reduct $\mathfrak{M}^{\prime}=\left\langle W^{\prime}, R^{\prime}, V^{\prime}\right\rangle$ in the same way as in Theorem 5.16. We take the symmetric-transitive closure $R^{*+}$ of $R^{\prime}$. By Theorem $5.19 R^{\prime}$ is reflexive, and so is $R^{\prime *+}$. By Theorem $5.20 \mathfrak{M}^{\prime}$ preserves the satisfaction of all formulas from $\operatorname{Sub}(\varphi)$ under the symmetric closure. By Theorem $5.22 \mathfrak{M}^{\prime}$ preserves the satisfaction of all formulas from $\operatorname{Sub}(\varphi)$ under the transitive closure. $\mathfrak{M}^{*+}$ is therefore a model for $\varphi$ and $R^{*+}$ is an equivalence relation. The conclusion follows.

Theorems 5.23 and 5.24 establish membership of $\mathcal{M}_{\mathrm{S} 5}\left(\mathrm{E}_{n}\right)$ and $\mathcal{M}_{\mathrm{K} 5}\left(\mathrm{E}_{n}\right)$ in NEXPTIME. Currently, we lack the lower complexity bound for these logics. To be more precise, we have NP-hardness for both $\mathcal{M}_{\mathrm{S} 5}\left(\mathrm{E}_{n}\right)$ and $\mathcal{M}_{\mathrm{K} 5}\left(\mathrm{E}_{n}\right)$, which follows from the fact that they both contain the ordinary propositional logic. These bounds, however, are 


\begin{tabular}{l|l} 
Frame class & Complexity class \\
\hline \hline arbitrary & NEXPTIME-complete \\
\hline reflexive & NEXPTIME-complete \\
\hline symmetric & NEXPTIME-complete \\
\hline serial & NEXPTIME-complete \\
\hline transitive & NEXPTIME-complete \\
\hline Euclidean & $\begin{array}{l}\text { in NEXPTIME } \\
\text { exponential-size model property } \\
\text { lack of polysize model property }\end{array}$ \\
\hline equivalential & $\begin{array}{l}\text { in NEXPTIME } \\
\text { exponential-size model property } \\
\text { lack of polysize model property }\end{array}$
\end{tabular}

Table 2: Complexity classes for the logic $\mathcal{M}\left(\mathrm{E}_{n}\right)$ defined over various frame classes.

not tight ${ }^{10}$. The following theorem of Areces et al. sheds some light on the possibility of polynomial reasoning for modal logics with global counting operators in which numerical subscripts are coded in binary.

THEOREM 5.25 ([6]). If numerical subscripts are coded in binary, then there are formulas in $\mathcal{M}\left(E_{n}\right)$ whose only models are exponentially larger.

This follows straightforwardly from the fact that under the binary coding assumption formulas of the form $\mathrm{E}_{=n} \top$ force the existence of models whose cardinality equals $n$, whereas the approximate size of such formulas is $\log (n)$. What we can derive is that even though we do not have the lower complexity bound for $\mathcal{M}_{55}\left(E_{n}\right)$ and $\mathcal{M}_{\mathrm{K} 5}\left(\mathrm{E}_{n}\right)$, we know that they do not enjoy the polysize model property. Even if it turned out that the actual complexity class for $\mathcal{M}_{\mathrm{S} 5}\left(\mathrm{E}_{n}\right)$ and $\mathcal{M}_{\mathrm{K} 5}\left(\mathrm{E}_{n}\right)$ is lower than NEXPTIME, we could not exploit this fact in tableau reasoning described in Chapters 2 and 3, where the complexity of the

$10 \overline{\text { Our strong conjecture is that both }} \mathcal{M}_{\mathrm{S} 5}\left(\mathrm{E}_{n}\right)$ and $\mathcal{M}_{\mathrm{K} 5}\left(\mathrm{E}_{n}\right)$ are NP-complete; nevertheless, at the moment we lack the formal proof of the membership of these logics in NP. 
whole procedure depends directly on the size of a model it can yield as a result.

The complexity results for modal logics with global counting operators are summarised in Table 2.

\subsection{HISTORICAL OVERVIEW}

Counting modalities were first introduced by Fine ([33]) in the early 1970s under the name of graded modalities. They allowed expressing a number of successors of a particular world, at which a certain formula holds. In particular, a formula $\nabla_{=n} \top$ was expressing the fact that the current world has exactly $n$ successors. It already enhanced the expressive power of modal logic to a significant extent. For instance, the functionality of a model, i.e., the fact that each world in the universe has exactly one successor, could be expressed by setting a formula $\nabla_{=1} T$ as an axiom. Some further developments of the theory of graded modalities, especially concerning axiomatizations for these logics and decidability results, were established by Fattorosi-Barnaba, Caro and Cerrato in the late 1980s and early 1990s, and can be found in $[23,25,32]$. Van der Hoek and de Rijke [98, 97] established graded modal logics as a modal tool for investigating first-order counting quantifiers and introduced the notion of propositional logic with counting $(\mathcal{P} \mathcal{L C})$ as a name for the logic S5 with graded modalities (see also [15]). In the field of modal logic, the topic of counting modalities was re-established in 2010 by Areces et al. in [6]. With the aim of improving the expressivity of modal logics, they introduced modal logics with counting operators, which they abbreviated as $\mathcal{M L C}$. Global counting operators $\mathbf{E}_{>n}, \mathbf{E}_{<n}$ and $\mathrm{E}_{=n}$ were added to a modal language with the ordinary modalities. The problem that remained open in [6] was the axiomatization for $\mathcal{M L C}$. It was resolved by the author of this book in [102] and recapitulated in this chapter. The other issue posed in [6] was the question of the complexity of $\mathcal{M L C}$ under the binary coding assumption. It was addressed in [104] and presented in an extended form in this chapter.

Simultaneously to modal logicians, the description logic community was developing tools dealing with practical aspects of reasoning with numerical restrictions. Tobies $([91,92])$ provided numerous results on 
the complexity of description logics with cardinality constraints coded in unary. Horrocks et al. ([43, 44, 45, 46, 47, 48, 49, 50, 51]) established various decision procedures for different description logics involving such constraints. The recent developments in description logics with cardinality constraints are due to Faddoul et al. and concern splitting decision procedures for these logics into the arithmetical and the logical part $([30,31])$. 

REMARKS ON DESCRIPTION LOGICS CONTRIBUTIONS

This chapter is aimed to provide a concise overview of basic notions used in description logics. Description and modal (and also hybrid) logics have a lot in common. In fact, the former can be considered as a notational variant of the latter. For that reason, references to works from the description logic field occur throughout the whole book. A brief explanation of a description logic terminology we refer to in our considerations is therefore in order.

First, let's recall that the basic modal logic is defined over a signature $\langle$ PROP, $R\rangle$, where PROP is a denumerable set of propositional variables $p_{1}, p_{2}, \ldots$ and $R$ is a binary relation over the universe of a model. Description logics use the notion of atomic concepts rather than propositional formulas. Let $\mathcal{C}_{0}$ denote a set of all atomic concepts of (an abstract) description logic $\mathcal{D}$. Again, in description logics we talk about roles rather than about accessibility relations. Let $\mathcal{R}$ denote a set of all roles of $\mathcal{D}$. We have all at hand to define a syntax of $\mathcal{D}$.

Definition 6.1 (Syntax of $\mathcal{D}$ ). Let $\mathcal{C}_{0}$ be the set of atomic concepts and $\mathcal{R}$ be the set of roles in $\mathcal{D}$. We define the set $\mathcal{C}$ of all concepts of the language of the description logic $\mathcal{D}$ as follows:

$$
\mathcal{C}:=\top|A| \neg C|C \sqcap D| C \sqcup D|\exists R . C| \forall R . C|n \geq R . C| n \leq R . C,
$$

where $A \in \mathcal{C}_{0}, C, D \in \mathcal{C}, R \in \mathcal{R}$ and $n \in \mathbb{N}$.

Before we draw a parallel between concrete modal and description notions, let's define a model for $\mathcal{D}$ which is called an interpretation.

Definition 6.2 (Semantics of $\mathcal{D}$ ). An interpretation $\mathcal{I}$ for the description logic $\mathcal{D}$ is a pair $\left\langle\triangle^{\mathcal{I}}, \mathcal{I}\right\rangle$, where $\triangle^{\mathcal{I}}$ is called a domain of interpretation and ${ }^{\mathcal{I}}$ is a function that maps all atomic concepts $A \in \mathcal{C}_{0}$ to subsets of $\triangle^{\mathcal{I}}$ and all roles $R \in \mathcal{R}$ to subsets of $\triangle^{\mathcal{I}} \times \triangle^{\mathcal{I}}$ and is 
called an interpretation function. ${ }^{\mathcal{I}}$ is homomorphically lifted-up to all concepts in the following way:

$$
\begin{array}{ll}
\top^{\mathcal{I}} & =\triangle^{\mathcal{I}}, \\
(\neg C)^{\mathcal{I}} & =\triangle^{\mathcal{I}} \backslash C^{\mathcal{I}}, \\
(C \sqcap D)^{\mathcal{I}} & =C^{\mathcal{I}} \cap C^{\mathcal{I}}, \\
(C \sqcup D)^{\mathcal{I}} & =C^{\mathcal{I}} \cap C^{\mathcal{I}} \\
(\exists R . C)^{\mathcal{I}} & =\left\{a \in \triangle^{\mathcal{I}} \mid \exists b\left(\langle a, b\rangle \in R^{\mathcal{I}} \wedge b \in C^{\mathcal{I}}\right)\right\}, \\
(\forall R . C)^{\mathcal{I}} & =\left\{a \in \triangle^{\mathcal{I}} \mid \forall b\left(\langle a, b\rangle \in R^{\mathcal{I}} \rightarrow b \in C^{\mathcal{I}}\right)\right\}, \\
(n \geq R . C)^{\mathcal{I}} & =\left\{a \in \triangle^{\mathcal{I}} \mid \operatorname{Card}\left(\left\{b \mid\langle a, b\rangle \in R^{\mathcal{I}} \wedge b \in C^{\mathcal{I}}\right\}\right) \geq n\right\}, \\
(n \leq R . C)^{\mathcal{I}} & =\left\{a \in \triangle^{\mathcal{I}} \mid \operatorname{Card}\left(\left\{b \mid\langle a, b\rangle \in R^{\mathcal{I}} \wedge b \in C^{\mathcal{I}}\right\}\right) \leq n\right\} .
\end{array}
$$

It is now clear that our logic $\mathcal{D}$ is a full analog of a multi-modal version of the logic $\mathcal{M}_{K}\left(E_{n}\right)$.

What is fresh in description logics is the so-called knowledge base (abbreviated as $K B$ ). Let's introduce this notion in a more formal fashion:

Definition 6.3 (Knowledge base). By an $A B o x_{\mathcal{D}}$ for the logic $\mathcal{D}$ we understand a set of assertions of the form $a: C$ or $\langle a, b\rangle: R$, where $a, b \in \triangle^{\mathcal{I}}, C \in \mathcal{C}$ and $R \in \mathcal{R}$. Intuitively, each of these statements assert that a certain individual from a domain satisfies a certain concept or that a pair of such individuals are in a relation.

By a $T B x_{\mathcal{D}}$ for the logic $\mathcal{D}$ we understand a set of concept inclusions of the form $C \sqsubseteq D$, where $C, D \in \mathcal{C}$. These inclusions determine which pairs of concepts are in a subsumption relation.

By an $R B o x_{\mathcal{D}}$ for the logic $\mathcal{D}$ we understand a set of role inclusions of the form $R \sqsubseteq S$, where $R, S \in \mathcal{R}$. These inclusions determine which pairs of roles are in a subsumption relation ${ }^{1}$.

By a knowledge base for the logic $\mathcal{D}$ we understand a set $K B=$ $A B o x_{\mathcal{D}} \cup T B o x_{\mathcal{D}} \cup R B o x_{\mathcal{D}}$.

Usually, reasoning in description logics focuses on determining whether a knowledge base is consistent. As we can see, description logics

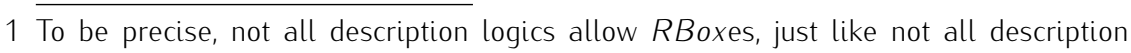
logics allow concepts to include numerical restrictions. However, in this section we draw the most general panorama of description logics possible. 


\begin{tabular}{c|c|l} 
Symbol & Operator & \multicolumn{1}{|c}{ Meaning } \\
\hline \hline $\mathcal{E}$ & $\exists R$ & full existential quantification \\
\hline $\mathcal{U}$ & $\sqcup$ & concept union \\
\hline $\mathcal{C}$ & $\neg$ & full concept negation \\
\hline $\mathcal{H}$ & $R \sqsubseteq S$ & role hierarchy \\
\hline $\mathcal{R}$ & $R \sqsubseteq S$ & limited complex role inclusions \\
\hline $\mathcal{O}$ & $o_{i}$ & nominals \\
\hline $\mathcal{I}$ & $R^{-}$ & inverse roles \\
\hline $\mathcal{N}$ & $n \geq, n \leq$ & unqualified numerical restrictions \\
\hline $\mathcal{Q}$ & $n \geq R, n \leq R$ & qualified numerical restrictions
\end{tabular}

Table 3: Symbols denoting various elements of description logic languages.

introduce hybrid language elements under the name of $A B o x$. The individuals occurring on the left-hand side of assertions of the form $a: C$ are, in fact, nominals and the assertions themselves are counterparts of hybrid satisfaction formulas @i $i$. The significant difference between $A B o x$ assertions and satisfaction formulas is that the former do not allow individuals to occur on the right hand-side ${ }^{2}$. As a consequence, we are not able to express the equality of two individuals or the fact that they are in a relation.

Description logics also allow for the explicit presence of nominals in their languages. However, there is no significant difference in behaviour between nominals in modal and description logics so we will not devote more space to a thorough explanation of the latter.

To conclude this section, we mention that even though names of particular description logics might seem odd to a modal logician, they are formed in a simple algorithmic manner. The basic description language is $\mathcal{A L C}$ (attributive language with full complement). If we want to augment our language with some operators or expressions, we simply add to the name of such a logic a letter ascribed to these elements. The description logic notation is displayed in Table 3. Additionally, $\mathcal{S}$ is an abbreviation for $\mathcal{A L C}$ with transitive roles. For an exhaustive introduction to description logics, see [8].

2 Provided that nominals are not explicitly introduced to the language of a logic. 



\section{Part II}

\section{DEDUCTIVE SYSTEMS FOR HYBRID LOGICS}

This part is devoted to concrete deductive systems for hybrid logics. The originally established tableau algorithms involving the unrestricted blocking mechanism are presented and serve as a starting point for a comparative study of existing decision procedures for hybrid logics. 

DEDUCTIVE SYSTEMS FOR STANDARD HYBRID LOGICS

In the first place, let's recall and briefly recapitulate some basic facts about hybrid logics that we use in the present chapter. Various issues related to hybrid logics are discussed more thoroughly in Chapter 4. Hybrid logics are powerful extensions of modal logics which allow for referring to particular states of a model without using meta-language. In order to achieve it, the language of standard modal logics is enriched with the countably infinite set of propositional expressions called nominals (we fix the notation $\mathrm{NOM}=\{i, j, k, \ldots\}$ to stand for the set of nominals), disjoint from the set of propositional variables PROP. Each nominal is true at exactly one world and therefore can serve both as a label and as a formula. Supplying a language with nominals significantly strengthens its expressive power. In the present chapter we consider a suitably modified hybrid logic obtained by adding the satisfaction operators, the universal modality, the difference modality and the inverse modality. As indicated in Chapter 4, the satisfaction operators of the form $@_{i}$ allow for stating that a particular formula holds at a world labelled by $i$, the universal modality $E$ expresses the fact that there exists a world in a domain, at which a particular formula holds, the difference modality D stands for the fact that a particular formula holds at a world different from the current one and, eventually, the inverse modality allows us to "jump back" to a predecessor-world along the accessibility relation edges.

As we mention in Section 4.2 of the preceding chapter, some hybrid logics additionally contain a different sort of expressions, the state variables, which allow quantifying over worlds, and additional operators like the $\downarrow$ operator or $\exists$. However, these logics are proven to be undecidable ([3]) so, in principle, they cannot be subjected to a terminating tableau-based decision procedure. We therefore hold our word from Chapter 4.3 and confine ourselves only to the foregoing decidable hybrid logic. According to the notation we set in the book, the symbol 
that should represent this logic is $\mathcal{H}\left(@, E, D, \diamond^{-}\right)$. However, due to its lengthy form, henceforth we refer to the logic as to the binder-free hybrid logic and denote it by $\mathcal{H}_{\text {b-f. }}$.

In the present chapter we introduce two sound, complete and terminating tableau calculi $\mathcal{T}_{\mathcal{H}_{\text {b-f }}}^{\mathrm{p}}$ and $\mathcal{T}_{\mathcal{H}_{\text {b-f }}}^{\mathrm{i}}$ for hybrid logics with @, E, D and $\diamond^{-}$operators. We can say that they are mutual analogs, the first of them being a prefixed calculus (which is capable of dealing with hybrid logics only containing nominals out of the whole hybrid toolkit), whereas the second being an internalized calculus. Our approach, unlike that in [40] and [16], is focused on the uniform treatment of all aforementioned logics, minimality of models generated by both $\mathcal{T}_{\mathcal{H}_{\text {b-f }}}^{\mathrm{p}}$ and $\mathcal{T}_{\mathcal{H}_{\text {b-f' }}}^{\mathrm{i}}$ and possible extensions of the calculus for different frame classes. Basing on [84], we exploit the unrestricted blocking mechanism that satisfies these conditions. As it occurs, blocking techniques involved in calculi presented in this chapter are the most significant distinctive features of them.

In Section 7.1 a general introduction into tableau calculi for hybrid logics is given. In Section 7.2 we introduce the prefixed tableau calculus $\mathcal{T}_{\mathcal{H}_{\text {b-f }}}^{\mathrm{p}}$ and prove soundness and completeness for $\mathcal{T}_{\mathcal{H}_{\text {b-f }}}^{\mathrm{p}}$. Also we

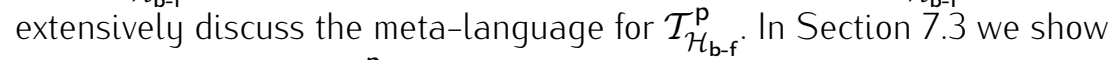
how certain rules of $\mathcal{T}_{\mathcal{H}_{\text {b-f }}}^{\text {p }}$ can be refined. Section 7.4 is devoted to the internalized calculus $\mathcal{T}_{\mathcal{H}_{\text {b-f }}^{\mathrm{i}-f}}$. A sequent calculus $\mathcal{S}_{\mathcal{H}_{\text {b-f }}}$ equivalent to $\mathcal{T}_{\mathcal{H}_{\text {b-f }}}^{\mathrm{i}}$ is introduced in Section 7.5. Termination of all calculi is demonstrated in Section 7.6. We allow ourselves to excurse towards a short overview of blocking mechanisms in other existing decision procedures in Section 7.7. Section 7.8 concludes the chapter.

We do not provide an exhaustive overview of existing deductive systems for hybrid logics since it can be found in a concise but very informative survey paper of Indrzejczak ([52]), and an extensive work on hybrid logics and the proof-theory for them of Braüner ([21]) 1 .

1 Sections 7.3, 7.4, 7.5 and 7.6 of the current chapter are reprinted from "Logic and Logical Philosophy", Vol. 22(4), Andrzej Indrzejczak, Michat Zawidzki, Decision procedures for some strong hybrid logics, Pages No. 389-409, Copyright (2013), with permission from Nicolaus Copernicus University Scientific Publishing House ([54]).

Some ideas shared in these sections appeared previously in the conference paper by the author of the book ([103]). 


\section{1 introduction to tableau CALCULI FOR the LOGIC $\mathcal{H}_{\text {B-F }}$}

Two main types of tableau calculi for hybrid logics are present in the literature, namely the prefixed and the internalized calculi. The prefixed calculi consist in introducing another sort of expressions, namely prefixes. They serve as labels for worlds, which, unlike nominals, are of meta-linguistic provenience. Another type of meta-language expressions occurring in prefixed tableaux are the accessibility expressions. The equality between two prefixes is expressed implicitly by imposing on them the satisfaction of the same nominal. Apparently, prefixed calculi are less complex than internalized calculi. Besides, basic hybrid logic $\mathcal{H}$ is not supplied with sufficient expressive power to internalize its own semantics. It therefore requires the domain expressions occurring in the calculus. The most widely known prefixed tableau calculi for hybrid logics come from Tzakova ([93]), Bolander and Braüner (who improved Tzakova's calculus to the terminating version) ([19]), Kaminski and Smolka ([57]). The tableau calculus for hybrid logics obtained from the synthesized framework from [84], which we present in the following part of the chapter, is also subsumed under the prefixed calculi class.

Internalized calculi for hybrid logics take advantage of the high expressive power of these logics which allows for encoding the domain expressions within the language. Although internalization of the logic allows for dispensing with certain rules present in prefixed tableau calculi, it also jeopardizes termination of the calculus by, e.g., using pure axioms (not including other formulas but nominals) to characterise frame conditions (cf. [16]).

In the remainder of the chapter we present both a prefixed and an internalized version of a tableau calculus covering hybrid logics with the satisfaction operators, the universal modality, the difference modality and the inverse modality. The internalized calculus resembles Blackburn's calculus from [10] modified by Bolander and Braüner in [19] and by Blackburn and Bolander in [16], although certain rules have been added (e.g. the rules for D). This, however, is jumping ahead. We start from presenting the prefixed version of the calculus since, unlike the internalized one, it is also suitable for the basic hybrid logic. 


\subsection{PREFIXED CALCULUS FOR $\mathcal{H}_{\text {B-F }}$}

\subsubsection{Meta-language for $\mathcal{H}_{b-f}$}

In this section we send the reader to Section 4.2 of Chapter 4 to take a glance at Definition 4.2 where the conditions of satisfaction for particular operators are presented. Since the logic we design our calculi for does not involve state variables, we can dispose of the valuation sign from the definition.

Our primary version of the calculus is prefixed, thus we shall start with a brief description of the whole meta-language machinery that is involved in a prefix algebra in our calculus. As we mentioned in Section 7.1 of this chapter, we exploit the tableau synthesis framework from [84], where the meta-language to specify the semantics of $\mathcal{H}_{\text {b-f }}$ is a many-sorted first-order language. (see [36]). Following [84] henceforth we denote it by $\mathrm{FO}\left(\mathcal{H}_{\mathrm{b}-\mathrm{f}}\right)$. A word of explanation needs to be devoted to the notion of a sort. Sorts of a particular language divide expressions of the language into distinct sets. In the case of modal logics, we can identify the sort of a particular expression $\varphi$ with the arity of first-order predicates resulting from the standard translation applied to $\varphi$. This means that nominals would have sort 0 as they translate to constants (nullary predicates), propositional variables would have sort 1 (as they become unary predicates after translation). Roles in description logics or programs in $\mathrm{PDL}^{2}$ would have sort 2 (since their first-order logic equivalents are binary relations). In fact, the above interpretation is used in [82] in regard to nominals. However, treating them as expressions of the sort 0 causes some difficulties if we want to make an explicit use of nominals as formulas. The remedy used in [82] is introducing additional unary operator $\{\cdot\}$, called the singleton set operator. It is only applicable to expressions of the sort 0 - nominals - and so applied it builds formulas of the form $\{i\}$ which are yet of the sort 1. As it turns out, this is the point where nominals reveal their hybrid nature, making the solution from [82] the only possible. We get back to this point after we present more extensively components of 
our many-sorted first-order meta-language of the hybrid logic under consideration.

For the object language of $\mathcal{H}_{\mathrm{b}-\mathrm{f}}$ we therefore need two sorts: 0 for nominals and 1 for formulas (expressions explicitly involving accessibility relations are not present in the language). The sorts of the metalanguage $\mathrm{FO}\left(\mathcal{H}_{\mathrm{b}-\mathrm{f}}\right)$ are then the sort 0 for nominals occurring in a hybrid formula, the sort 1 for formulas of $\mathcal{H}_{\mathrm{b}-\mathrm{f}}$ and the sort 2 as the domain sort. Expressions of sort 2 relate to the elements of the firstorder domain (under a particular valuation). $\mathrm{FO}\left(\mathcal{H}_{\mathrm{b}-\mathrm{f}}\right)$ as a first-order language is equipped with the usual logical constants and expressions. Furthermore, it contains the following symbols as first-order equivalents for $\mathcal{H}_{\text {b-f-expressions: }}$

1. functional symbols obtained from the connectives of $\mathcal{H}_{\mathrm{b}-\mathrm{f}}$ : unary functional symbols $\neg, \diamond, E, D, \diamond^{-}$(sort $1 \longrightarrow 1$ ), and binary functional symbol $\wedge$ (sort $1,1 \longrightarrow 1)$,

2. additional operator $\{\cdot\}$ of the sort $0 \longrightarrow 1$, whose meaning is explained in the following part of the section,

3. a constant binary predicate symbol $R(\operatorname{sort}(2,2))$,

4. the equality symbol $\approx(\operatorname{sort}(2,2))$,

5. interpretation function symbol $v_{0}($ sort $0 \longrightarrow 2)$,

6. interpretation predicate symbol $v_{1}($ sort $(1,2))$.

In the semantic specification $\mathcal{H}_{\mathrm{b}-\text { f }}$ formulas (of sort 1 ) are treated as $\mathrm{FO}\left(\mathcal{H}_{\text {b-f }}\right)$-terms. The $v_{1}$ predicate can be viewed as representing the $\Vdash$ relation, which means that the formula $v_{1}(\varphi, x)$ expresses $\mathfrak{M}, w \Vdash \varphi . v_{0}$ is a function symbol taking as its sole argument a nominal and switching it into a sort 2 term. $v_{i}$ symbols, $i \geq 1$, are $i+1$-ary predicates applicable to object-language expressions of the sort $i$. We therefore have only one $v$ predicate: $v_{1}$ which is a binary relation with a modal formula standing in place of the first argument and a domain sort expression as the second argument.

The atomic expressions of $\mathrm{FO}\left(\mathcal{H}_{\mathrm{b}-\mathrm{f}}\right)$ are terms of the form $v_{0}(i)$, for all terms $i$ (sort 0 ), and formulas of the form $v(\varphi, x), R(w, v)$ and $x \approx y$, for all terms $\varphi$ (sort 1), $x, y$ (sort 2). 
Now, let's jump back to the problem of the hybrid nature of nominals. Let's once again have a look at the standard translation conditions for nominals and satisfaction formulas from Section 4.2 of Chapter 4 :

$$
\begin{aligned}
& \mathrm{ST}_{x}(i)=x=x_{i}, \quad i \in \mathrm{NOM}, \\
& \mathrm{ST}_{x}\left(@_{i} \varphi\right)=\left(\mathrm{ST}_{x}(\varphi)\right)\left[x_{i} / x\right] .
\end{aligned}
$$

As we can see, nominals are translated to first-order constants. This is necessary to preserve their semantics of expressions that only hold at one world ${ }^{3}$. Unfortunately, as expressions of the sort 0 nominals cannot be treated as formulas and, correspondingly, they cannot be evaluated at particular worlds (this is represented by $v_{1}$ in our calculus and it only takes formulas as its first argument). As we can see, introducing another operator that would turn nominals-labels into nominals-formulas is indispensable. Hence, we augment our object language with the $\{\cdot\}$ operator, which is appropriately reflected in the calculus (see Fig. 13).

The foregoing instruments are sufficient to give a full semantic specification of $\mathcal{H}_{\text {b-f }}$ in $\mathrm{FO}\left(\mathcal{H}_{\text {b-f }}\right)$. We can look upon the semantic specification of a certain logic as a set of axioms for a class of first-order structures where each connective from the logic is unambiguously defined (see [84]). It consists of two parts: the definitions of the connectives $\left(S^{0}\right)$ and the background theory $\left(S^{b}\right)$.

The $\mathrm{FO}\left(\mathcal{H}_{\mathrm{b}-\mathrm{f}}\right)$ definitions for the connectives are easily obtained from Definition 5.2 by replacing $\mathfrak{M}, w \Vdash \varphi$ by $v_{1}(\varphi, w)$ and meta-linguistic connectives by $\mathrm{FO}\left(\mathcal{H}_{\mathrm{b}-\mathrm{f}}\right)$-connectives, which are shown in Fig. 10. All formulas are universally quantified. In order to obtain tableau rules for the connectives, we divide $S^{0}$ into two disjoint sets of formulas $\left(S_{+}^{0}\right.$ and $\left.S_{-}^{0}\right)$ and write them in implicational form as given in Fig. 11. Obtaining $S_{-}^{0}$ involves taking the contrapositives of the right-to-left implications of the formulas in $S^{0}$, turning them into prenex normal form and eliminating quantifiers via Skolemization.

The background theory provides a frame characterisation for the considered logic. If we consider the logic $K$, the background theory only contains equality axioms. Since we want to make the calculus as extensive as possible, we also formulate the conditions for various frame

3 If we want to handle them as formulas in our calculus, it would lead to undesirable consequences. For instance, as one can easily check by a short glance at Figs 20 and 21, we would no longer be able to preserve self-duality of @. 


$$
\begin{aligned}
& \mathrm{S}^{0}: \forall x\left(v_{1}(\{i\}, x) \equiv v_{0}(i) \approx x\right) \\
& \forall x\left(v_{1}(\neg \varphi, x) \equiv \neg v_{1}(\varphi, x)\right) \\
& \forall x\left(v_{1}(\varphi \wedge \psi, x) \equiv\left(v_{1}(\varphi, x) \wedge v(\psi, x)\right)\right) \\
& \forall x\left(v_{1}(\diamond \varphi, x) \equiv \exists y\left(R(x, y) \wedge v_{1}(\varphi, y)\right)\right) \\
& \forall x\left(v_{1}\left(@_{i} \varphi, x\right) \equiv v_{1}\left(\varphi, v_{0}(i)\right)\right) \\
& \forall x\left(v_{1}(\mathrm{E} \varphi, x) \equiv \exists y\left(v_{1}(\varphi, y)\right)\right) \\
& \forall x\left(v_{1}(\mathrm{D} \varphi, x) \equiv \exists y\left(v_{1}(\varphi, y) \wedge x \neq y\right)\right) \\
& \forall x\left(v_{1}\left(\diamond^{-} \varphi, x\right) \equiv \exists y\left(R(y, x) \wedge v_{1}(\varphi, y)\right)\right)
\end{aligned}
$$

Figure 10: Semantic specification of connectives $S^{0}$ for the logic $\mathcal{H}_{\text {b-f }}$.

classes. The background theory conditions are given in the usual manner in a universally quantified form. Obtaining tableau rules for the background theory involves eliminating quantifiers by using Skolemization. In the presentation of the background theory in Fig. 12 quantifiers have already been eliminated.

The specification in Figs 11 and 12 is normalized as defined in [84].

\subsubsection{Tableau calculus $\mathcal{T}_{\mathcal{H}_{b-f}}^{p}$}

The tableau calculus synthesized from the semantic specification provided in the previous section in Figs 11 and 12 is given in Fig. 13. We refer to it as $\mathcal{T}_{\mathcal{H}_{\text {b-f }}}^{\text {p }}$ (prefixed tableau calculus for the logic $\mathcal{H}_{\text {b-f }}$ ).

Intuitively, an expression of the form $v_{1}(\varphi, x)$ means that a formula $\varphi$ holds at a world $x$, and $\neg v_{1}(\varphi, x)$ means the contrary. If a rule is a multi-conclusion rule, we enumerate in the denominator all conclusions using commas. If a rule is branching, we write in the denominator all alternative conclusions, separating them with | signs. Given $\Gamma$, the input set of formulas, we start the derivation putting at the initial node of the tableau all formulas from the set $\left\{v_{1}(\varphi, x) \mid \varphi \in \Gamma\right\}$ where $x$ is a fresh constant of the domain sort.

The tableau rules for the Boolean connectives are quite straightforward. However, two different negation signs occur in the rule $(\neg)$, the one in the premise denoting $\mathcal{H}_{\mathrm{b}-\mathrm{f}}$-negation, and the one occurring in the conclusion denoting $\mathrm{FO}\left(\mathcal{H}_{\mathrm{b}-\mathrm{f}}\right)$-negation. 


$$
\begin{aligned}
& \mathrm{S}_{+}^{0}: v_{1}(\{i\}, x) \quad \rightarrow \quad v_{0}(i) \approx x \\
& v_{1}(\neg \varphi, x) \quad \rightarrow \quad \neg v_{1}(\varphi, x) \\
& \left.v_{1}(\varphi \wedge \psi, x) \rightarrow\left(v_{1}(\varphi, x) \wedge v_{1}(\psi, x)\right)\right) \\
& v_{1}(\diamond \varphi, x) \rightarrow\left(R(x, f(\diamond \varphi, x)) \wedge v_{1}(\varphi, f(\diamond \varphi, x))\right) \\
& v_{1}\left(@_{i} \varphi, x\right) \rightarrow v_{1}\left(\varphi, v_{0}(i)\right) \\
& v_{1}(\mathrm{E} \varphi, x) \quad \rightarrow \quad v_{1}(\varphi, f(\mathrm{E} \varphi, x)) \\
& v_{1}(\mathrm{D} \varphi, x) \rightarrow\left(v_{1}(\varphi, f(\mathrm{D} \varphi, x)) \wedge x \neq f(\mathrm{D} \varphi, x)\right) \\
& v_{1}\left(\diamond^{-} \varphi, x\right) \rightarrow\left(R\left(f\left(\diamond^{-} \varphi, x\right), x\right) \wedge v_{1}\left(\varphi, f\left(\diamond^{-} \varphi, x\right)\right)\right) \\
& \mathrm{S}_{-}^{0}: \neg v_{1}(\{i\}, x) \rightarrow v_{0}(i) \neq x \\
& \neg v_{1}(\neg \varphi, x) \quad \rightarrow \quad v_{1}(\varphi, x) \\
& \neg v_{1}(\varphi \wedge \psi, x) \quad \rightarrow \quad\left(\neg v_{1}(\varphi, x) \vee \neg v_{1}(\psi, x)\right) \\
& \neg v_{1}(\diamond \varphi, x) \quad \rightarrow \quad\left(\neg R(x, y) \vee \neg v_{1}(\varphi, y)\right) \\
& \neg v_{1}\left(@_{i} \varphi, x\right) \rightarrow \neg v_{1}\left(\varphi, v_{0}(i)\right) \\
& \neg v_{1}(\mathrm{E} \varphi, x) \quad \rightarrow \quad \neg v_{1}(\varphi, y) \\
& \neg v_{1}(\mathrm{D} \varphi, x) \quad \rightarrow \quad\left(\neg v_{1}(\varphi, y) \vee x \approx y\right) \\
& \neg v_{1}\left(\diamond^{-} \varphi, x\right) \quad \rightarrow \quad\left(\neg R(y, x) \vee \neg v_{1}(\varphi, y)\right)
\end{aligned}
$$

Figure 11: Sets $S_{+}^{0}$ and $S_{-}^{0}$ of Skolemized rules for connectives.

The rule $(\{\cdot\})$ equates the interpretation of $i$ with the world $x$ at which $\{i\}$ holds. $(\neg\{\cdot\})$ introduces the interpretation of $i$ as a label and states that the world at which $\neg\{i\}$ holds is not equal to $v_{0}(i)$. Note that it does not introduce a formula $\{i\}$ to a branch. This can be dispensed with since equality reasoning handles nominals (occurring both explicitly as $\{\cdot\}$ formulas, and as labels for particular worlds). The rules $(\diamond)$ and $\left(\diamond^{-}\right)$operate in a standard way. In this version of the tableau calculus, the rules $(\neg \diamond)$ and $\left(\neg \nabla^{-}\right)$are branching but in Section 8.3 we prove that it is possible to turn the $\neg R(x, y)$ conclusion into a premise and thus dispose of branching involved in this rule. The occurrence of the $y \approx y$ formula in the $(\neg \diamond)$ rule explicitly ensures that the rule is applied only to the worlds that occur on the current branch (and to them only). The (@) and ( $@ @$ ) rules distribute a formula under the scope of @i (or, respectively, its negation) to a world which is an interpretation of a nominal $i$. The $(\mathrm{E})$ rule simply introduces a new 


$$
\begin{aligned}
& \mathrm{S}^{\mathrm{b}}: x \approx x \quad x \approx y \rightarrow y \approx x \quad x \approx y \wedge y \approx z \rightarrow x \approx z \\
& \left(x \approx y \wedge v_{1}(\varphi, x)\right) \rightarrow v_{1}(\varphi, y) \quad x \approx y \rightarrow f(\varphi, x) \approx f(\varphi, y) \\
& (x \approx y \wedge R(x, z)) \rightarrow R(y, z) \quad(x \approx y \wedge R(z, x)) \rightarrow R(z, y) \\
& \text { (ref) } x \approx x \rightarrow R(x, x) \quad(\text { sym) } R(x, y) \rightarrow R(y, x) \\
& \text { (ser) } x \approx x \rightarrow R(x, f(x \approx x, x)) \quad(\operatorname{tran})(R(x, y) \wedge R(y, z)) \rightarrow R(x, z) \\
& \text { (Eucl) } R(x, y) \wedge R(x, z) \rightarrow R(y, z)
\end{aligned}
$$

Figure 12: Semantic specification of background theory $S^{b}$ for $\mathcal{H}_{\mathrm{b}-\mathrm{f}}$.

world at which $\varphi$ holds. ( $\neg \mathrm{E})$ determines that at no world $y$ already present on a branch a formula under the scope of $E$ holds. Again, we use $y \approx y$ formulas to ensure that the rule is applied only to domain terms occurring on the current branch. The positive rule for $D$ introduces a new world to a branch, distinct from the current one, at which a formula under the scope of $D$ holds. $(\neg D)$ states that for each world $y$ present on a branch it is either the case that a formula under the scope of $D$ does not hold at $y$, or $y$ is equal to the current world. The negative rule for $D$ is also splitting, which might appear odd. In most tableau calculi, if we want to turn one of the conclusions into premise, we loose the completeness of a calculus. Like for the $(\neg \diamond)$, however, we show that we can refine $(\neg D)$ to a non-branching version. In fact, to our best knowledge, it is the first tableau calculus involving $D$ in the literature, in which such a shift was proven possible.

In the positive rules for $\diamond$ and $E, D$ and $\diamond^{-}$after eliminating existential quantifiers, instead of introducing a fresh constant, a Skolem term of the form $f(\varphi, x)$ is used. The advantage of this approach is fewer rule applications during the derivation. If, for example, at a certain point in a derivation we obtain the formula $x \approx f(\varphi, x)$, by the appropriate equality rules we also automatically obtain $x \approx f(\varphi, f(\varphi, x))$, whereas this would not be possible if we use fresh constants.

The first three rules for equality express the fact that it is an equivalence relation. The remaining four rules state congruence of $\approx$ with respect to $v$ and $R$ predicates and Skolem functions. 
Rules for connectives:

$$
\begin{aligned}
& (\{\cdot\}) \frac{v_{1}(\{i\}, x)}{v_{0}(i) \approx x}(\neg\{\cdot\}) \frac{\neg v_{1}(\{i\}, x)}{x \neq v_{0}(i), v_{0}(i) \approx v_{0}(i)} \quad(\neg) \frac{v_{1}(\neg \varphi, x)}{\neg v_{1}(\varphi, x)} \quad(\neg \neg) \frac{\neg v_{1}(\neg \varphi, x)}{v_{1}(\varphi, x)} \\
& (\wedge) \frac{v_{1}(\varphi \wedge \psi, x)}{v_{1}(\varphi, x), v_{1}(\psi, x)} \quad(\neg \wedge) \frac{\neg v_{1}(\varphi \wedge \psi, x)}{\neg v_{1}(\varphi, x) \mid \neg v_{1}(\psi, x)} \\
& (\diamond) \frac{v_{1}(\nabla \varphi, x)}{R(x, f(\diamond \varphi, x)), v_{1}(\varphi, f(\diamond \varphi, x))} \quad(\neg \diamond) \frac{\neg v_{1}(\nabla \varphi, x), y \approx y}{\neg R(x, y) \mid \neg v_{1}(\varphi, y)} \\
& \text { (@) } \frac{v_{1}\left(@_{i} \varphi, x\right)}{v_{1}\left(\varphi, v_{0}(i)\right)} \quad(\neg @) \frac{\neg v_{1}\left(@_{i} \varphi, x\right)}{\neg v_{1}\left(\varphi, v_{0}(i)\right)} \\
& \text { (E) } \frac{v_{1}(\mathrm{E} \varphi, x)}{v_{1}(\varphi, f(\mathrm{E} \varphi, x))} \quad(\neg \mathrm{E}) \frac{\neg v_{1}(\mathrm{E} \varphi, x), y \approx y}{\neg v_{1}(\varphi, y)} \\
& \text { (D) } \frac{v_{1}(\mathrm{D} \varphi, x)}{v_{1}(\varphi, f(\mathrm{D} \varphi, x), x \not f f(\mathrm{D} \varphi, x))} \quad(\neg \mathrm{D}) \frac{\neg v_{1}(\mathrm{D} \varphi, x), y \approx y}{\neg v_{1}(\varphi, y) \mid x \approx y} \\
& \left(\diamond^{-}\right) \frac{v_{1}\left(\diamond^{-} \varphi, x\right)}{R\left(f\left(\diamond^{-} \varphi, x\right), x\right), v_{1}\left(\varphi, f\left(\diamond^{-} \varphi, x\right)\right)} \quad\left(\neg \diamond^{-}\right) \frac{\neg v_{1}\left(\diamond^{-} \varphi, x\right), y \approx y}{\neg R(y, x) \mid \neg v_{1}(\varphi, y)}
\end{aligned}
$$

Rules for equality:

$$
\begin{gathered}
\frac{v_{1}(\varphi, x)}{x \approx x} \quad \frac{x \approx y}{y \approx x} \quad \frac{x \approx y, y \approx z,}{x \approx z} \quad \frac{x \approx y, R(x, z)}{R(y, z)} \quad \frac{x \approx y, R(z, x)}{R(z, y)} \\
\frac{x \approx y, v_{1}(\varphi, x)}{v_{1}(\varphi, y)} \quad \frac{x \approx y}{f(\varphi, x) \approx f(\varphi, y)}
\end{gathered}
$$

Closure rules and unrestricted blocking rule:

$$
\frac{v_{1}(\varphi, x), \neg v_{1}(\varphi, x)}{\perp} \quad \frac{R(x, y), \neg R(x, y)}{\perp} \quad \frac{x \approx y, x \neq y}{\perp} \quad \text { (ub) } \frac{x \approx x, y \approx y}{x \approx y \mid x \neq y}
$$

Rules for frame conditions:

$$
\begin{gathered}
\text { (ref) } \frac{x \approx x}{R(x, x)} \quad(\text { sym }) \frac{R(x, y)}{R(y, x)} \quad(\operatorname{tran}) \frac{R(x, y), R(y, z)}{R(x, z)} \\
\text { (Eucl) } \frac{R(x, y), R(x, z)}{R(y, z)} \quad(\text { ser }) \frac{x \approx x}{R(x, f(x, x \approx x))}
\end{gathered}
$$

Figure 13: The rules of $\mathcal{T}_{\mathcal{H}_{\mathrm{b}-\text { f }}}^{\mathrm{p}}$. 
The closure rules and the frame rules are self-evident and require no explanation.

The (ub) rule is a special variant of the analytical cut rule which was sucessfully applied to reduce a branching factor and, in consequence, the size of proofs in the tableau-like calculus KE (cf. [26]). While applying full analytic cut, we can divide any branch with respect to a subformula and its negation of any formula already occurring on a branch. (ub) is more restrictive since it is applied only to domain sort expressions. Intuitively, if two domain sort expressions (labels) appear on a branch, they either label two distinct worlds or the same world. Thus, (ub) allows comparing any pair of labels that appeared on a branch. As it will turn out before long, this possibility is essential for termination of the whole calculus. The main rationale behind introduction of $(\mathrm{ub})$ is connected with termination problems. It is an explicit rule-realization of unrestricted blocking strategy. However, it appears that the introduction of this rule leads also to some other advantages connected with the form of rules for $\mathrm{D}$ and reduction of the branching factor, on which we elaborate in Section 7.3.

\subsubsection{Soundness and completeness of $\mathcal{T}_{\mathcal{H}_{b-f}}^{p}$}

In this section we prove soundness and completeness of $\mathcal{T}_{\mathcal{H}_{\mathbf{b}-\mathbf{f}}}^{\mathrm{p}}$. In general, a tableau calculus $\mathcal{T}$ is sound iff for each satisfiable input formula $\varphi$ each tableau $\mathcal{T}(\varphi)$ is open, i.e. there exists a fully expanded branch in which no closure rule was applied. A tableau calculus is called complete iff for each unsatisfiable input formula $\varphi$ there exists a closed tableau, i.e. a tableau where each branch is closed. We call a branch of a tableau closed if the closure rule was applied on it. If a branch is not closed, it is open. An open branch is fully expanded if no other rules are applicable on it.

Since each rule of the calculus preserves satisfiability, which can be proven by easy verification, we obtain:

TheOREM 7.1. The tableau calculus $\mathcal{T}_{\mathcal{H}_{b-f}}^{p}$ is sound.

The calculus $\mathcal{T}_{\mathcal{H}_{b-f}}^{\text {p }}$ is also constructively complete and, therefore, complete. Before we give a formal definition of constructive complete- 
ness and prove that $\mathcal{T}_{\mathcal{H}_{\text {b-f }}}^{\mathrm{p}}$ has this property, we will introduce several preliminary notions.

For an open, fully expanded branch $\mathcal{B}$, let $\sim_{\mathcal{B}}$ be a relation defined as follows:

$$
x \sim_{\mathcal{B}} y \text { iff } x \approx y \in \mathcal{B} .
$$

Because all the equality rules are applied on the branch, $\sim_{\mathcal{B}}$ is an equivalence relation.

For a model $\mathfrak{M}=(W, R, V)$ let $\gamma: \mathrm{FO}\left(\mathcal{H}_{\mathrm{b}-\mathrm{f}}\right) \rightarrow W \cup \mathrm{FO}\left(\mathcal{H}_{\mathrm{b}-\mathrm{f}}\right)$ be a function that maps each propositional variable of the sort 1 to itself and each term of the domain sort to an element of a domain $W$ of a model $\mathfrak{M}$. We say that a model $\mathfrak{M}$ reflects a formula $\varphi$ of the sort 1 that occurred on a branch $\mathcal{B}$ if the following two conditions are true:

(i) if $v_{1}(\varphi, x) \in \mathcal{B}$ then $\mathfrak{M}, \gamma(x) \Vdash \varphi$,

(ii) if $\neg v_{1}(\varphi, x) \in \mathcal{B}$ then $\mathfrak{M}, \gamma(x) \| \varphi$.

We say that $\mathfrak{M}$ reflects $\mathcal{B}$ iff it reflects all formulas of the sort 1 that appear in $\mathcal{B}$.

It is worth noting that, in general, $v_{1}(\varphi, x) \in \mathcal{B}$ and $\neg v_{1}(\varphi, x) \in \mathcal{B}$ are not complementary cases and there are tableau derivations where neither of such terms occur on a branch.

Definition 7.1. We call a tableau calculus $\mathcal{T}$ constructively complete iff for each input formula $\varphi$, for any open, fully expanded branch $\mathcal{B}$ in a tableau $\mathcal{T}(\varphi)$ there exists a model $\mathfrak{M}$ such that:

(i) the domain $W$ of $\mathfrak{M}$ is defined as follows: $W=\left\{[x]_{\sim_{\mathcal{B}}} \mid x \approx x \in \mathcal{B}\right\}$,

(ii) $\mathfrak{M}$ reflects $\mathcal{B}$ under the canonical projection valuation $\pi$ defined as follows: $\pi(x)=[x]_{\sim_{\mathcal{B}}}$ for every term $x$ of the domain sort that appeared in $\mathcal{B}$.

Now, suppose that $\varphi$ is a formula. Let $\varphi$ be an input for our tableau calculus $\mathcal{T}_{\mathcal{H}_{\text {b-f }}}^{\mathrm{p}}$. We denote a tableau for $\varphi$ by $\mathcal{T}_{\mathcal{H}_{\text {b-f }}}^{\mathrm{p}}(\varphi)$. Let $\mathcal{B}$ be an open, fully-expanded branch of $\mathcal{T}_{\mathcal{H}_{\text {b-f }}}^{\mathrm{p}}(\varphi)$. We associate with $\mathcal{B}$ a model $\mathfrak{M}(\mathcal{B})=\langle\mathrm{W}, \mathrm{R}, \mathrm{V}\rangle$ defined as follows

$\mathrm{W}=\{\pi(x) \mid x$ is a term of the domain sort and $x \approx x \in \mathcal{B}\}$, 
$\mathbf{R}=\{\langle\pi(x), \pi(y)\rangle \mid x, y$ are terms of the domain sort and $R(x, y) \in$ $\mathcal{B}\}$,

$V$ maps every propositional variable $p$ of the sort 1 to the set $\{\pi(x) \mid$ $\left.v_{1}(p, x) \in \mathcal{B}\right\}$ and every formula of the form $\{i\}$ to $\pi\left(v_{0}(i)\right)$ such that $v_{0}(i) \approx v_{0}(i) \in \mathcal{B}$.

Lemma 7.2. Let $\varphi$ be an input $\mathcal{H}_{\mathrm{b}-\mathrm{f}}$-formula. Let $\mathcal{B}$ be an open, fully expanded branch of a tableau for $\varphi$. Then $\mathfrak{M}(\mathcal{B})$ reflects $\mathcal{B}$, that is:

(i) for any $\mathcal{H}_{\text {b-f }}$ formula $\varphi$, if $v_{1}(\varphi, x) \in \mathcal{B}$ then $\mathfrak{M}(\mathcal{B}), \pi(x) \Vdash \varphi$,

(ii) if $R(x, y) \in \mathcal{B}$ then $\langle\pi(x), \pi(y)\rangle \in \mathbf{R}$,

(iii) if $x \approx y$ then $\pi(x)=\pi(y)$.

Proof. Since (ii) and (iii) follow directly from the construction of $\mathrm{R}$, the definition of $\mathfrak{M}(\mathcal{B})$ and the construction of $W$ respectively, we confine ourselves to the proof of (i). We proceed by induction on the complexity of $\varphi$.

$\varphi=p$. Since $v_{1}(p, x) \in \mathcal{B}$, by definition of $\mathrm{V}$ we obtain that $\pi(x) \in \mathrm{V}(p)$, whence $\mathfrak{M}(\mathcal{B}), \pi(x) \Vdash p$.

$\varphi=\{i\}$. By application of the $(\{i\})$ rule to $v_{1}(\{i\}, x)$, we obtain $x \approx v_{0}(i) \in \mathcal{B}$. By applying the rules for $\approx$ we obtain $v_{0}(i) \approx v_{0}(i)$. By the definition of $\mathrm{V}$ we obtain $\mathfrak{M}, \pi(x) \Vdash\{i\}$.

$\varphi=\neg \psi$. We consider the following cases:

$\psi=p . \quad v_{1}(\neg p, x)$ occurred on $\mathcal{B}$. Since $\mathcal{B}$ is fully expanded, we have that $(\neg)$ has been applied to $v_{1}(\neg p, x)$. Thus, $\neg v_{1}(p, x)$ occurred on $\mathcal{B}$. By the definition of $\mathrm{V}$ and $\Vdash$ we obtain $\mathfrak{M}(\mathcal{B}) \| p$, whence $\mathfrak{M}(\mathcal{B}) \Vdash \neg p$.

$\psi=\{i\} . \quad v_{1}(\neg\{i\}, x)$ occurred on $\mathcal{B}$. Since $\mathcal{B}$ is fully expanded, we have that $(\neg)$ and $(\neg\{i\})$ have been applied to $v_{1}(\neg\{i\}, x)$. Thus, $v_{0}(i) \neq x \in \mathcal{B}$ and $v_{0}(i) \approx v_{0}(i) \in \mathcal{B}$ hold. Since the formula $x \approx v_{0}(i)$ has not appeared on $\mathcal{B}$, it is not the case that $x \sim_{\mathcal{B}} v_{0}(i)$. By the inductive hypothesis we therefore obtain that there exists a world $\pi\left(v_{0}(i)\right)$ at which $\{i\}$ holds and that $\pi(x) \neq \pi\left(v_{0}(i)\right)$. 
$\psi=\neg \chi$. We have that $v_{1}(\neg \neg \chi, X) \in \mathcal{B}$. Branch $\mathcal{B}$ is fully expanded so the rules $(\neg)$ and $(\neg \neg)$ must have been already applied to $\varphi=\neg \neg X$. Hence $v_{1}(X, x) \in \mathcal{B}$. By the inductive hypothesis $\mathfrak{M}(\mathcal{B}), \pi(x) \Vdash \neg \neg X$.

$\psi=x \wedge \theta . \quad v_{1}(\neg(x \wedge \theta), x)$ occurred on $\mathcal{B}$. Because $\mathcal{B}$ is fully expanded, we have that $(\neg)$ and $(\neg \wedge)$ have been applied to $v(\neg(x \wedge \theta), x)$. Thus, either $\neg v_{1}(x, x) \in \mathcal{B}$ or $\neg v_{1}(\theta, x) \in \mathcal{B}$ holds. Suppose that the former is the case. Then, by the inductive hypothesis, $\mathfrak{M}(\mathcal{B}), \pi(x) \Vdash \neg \chi$. It follows that $\mathfrak{M}(\mathcal{B}), \pi(x) \Vdash \neg \chi$ or $\mathfrak{M}(\mathcal{B}), \pi(x) \Vdash \neg \theta$, so by the definition of $\mathfrak{M}(\mathcal{B})$ we derive $\mathfrak{M}(\mathcal{B}), \pi(x) \Vdash \neg(x \wedge \theta)$. The latter can be proven similarly.

$\psi=\diamond x$. We have that $v_{1}(\neg \diamond x, x) \in \mathcal{B}$. If there is no $y$ such that $y \approx y \in \mathcal{B}$ and $\langle\pi(x), \pi(y)\rangle \in \mathrm{R}$, then the case is trivial. Let $\pi(y)$ be an arbitrary element of $\mathrm{W}$ such that $\langle\pi(x), \pi(y)\rangle \in \mathbf{R}$. By construction of $\mathrm{R}$ there are terms $u, v$ such that $R(u, v) \in \mathcal{B}$, $x \approx u \in \mathcal{B}, y \approx v \in \mathcal{B}$. Since $\mathcal{B}$ is open and fully expanded, by the equality rules, we have that $R(x, y) \in \mathcal{B}$. Thus, by the rule $(\neg)$ and $(\neg \diamond)$, we obtain $\neg v_{1}(x, y)$. By the inductive hypothesis we have $\mathfrak{M}(\mathcal{B}), \pi(y) \Vdash x$, whence $\mathfrak{M}(\mathcal{B}), \pi(y) \Vdash \neg \chi$. Because $\pi(y)$ was arbitrarily chosen, we have $\mathfrak{M}(\mathcal{B}), \pi(x) \Vdash \neg \diamond x$.

$\psi=@_{i} X$. We have that $v_{1}\left(\neg @_{i} X, x\right) \in \mathcal{B}$. B is open and fullyexpanded, thus $(\neg)$ and ( $\neg @$ ) have been applied to our formula. It means that $\neg v_{1}\left(X, v_{0}(i)\right) \in \mathcal{B}$. By the inductive hypothesis $\mathfrak{M}, \pi\left(v_{0}(i)\right) \Vdash X$, whence $\mathfrak{M}, \pi\left(v_{0}(i)\right) \Vdash \neg \chi$. By the definition of $\Vdash$, we obtain that $\mathfrak{M}, \pi(x) \Vdash \neg @ i X$.

$\psi=\mathrm{E}_{X}$. We have that $v\left(\neg \mathrm{E}_{X}, x\right) \in \mathcal{B}$. Let $\pi(y) \in \mathrm{W}$. It follows that $y \approx y \in \mathcal{B}$. Since the rules $(\neg)$ and $(\neg E)$ have been applied to $v_{1}\left(\neg \mathrm{E}_{X}, x\right)$ on $\mathcal{B}$ we have $\neg v_{1}(X, y) \in \mathcal{B}$. By the inductive hypothesis we have $\mathfrak{M}(\mathcal{B}), \pi(y) \Vdash \chi$, whence $\mathfrak{M}(\mathcal{B}), \pi(y) \Vdash \neg \chi$. Since $\pi(y)$ was picked arbitrarily from $W$, by the definition of $\mathfrak{M}(\mathcal{B})$ we obtain $\mathfrak{M}(\mathcal{B}), \pi(x) \Vdash \neg \mathrm{E} X$.

$\psi=\mathrm{D} X$. Let $\pi(y) \in \mathrm{W}$. It follows that $y \approx y \in \mathcal{B}$. Since the rules $(\neg)$ and $(\neg \mathrm{D})$ have been applied to $v_{1}(\neg \mathrm{D} X, x)$ on $\mathcal{B}$, we have $\neg v_{1}(x, y) \in \mathcal{B}$ or $x \approx y \in \mathcal{B}$. If the former is the case, by the inductive hypothesis we get $\mathfrak{M}(\mathcal{B}), \pi(y)$ \& $\chi$, whence 
$\mathfrak{M}(\mathcal{B}), \pi(y) \Vdash \neg \chi$. If the latter is the case, then by construction of $\mathfrak{M}(\mathcal{B})$ we obtain $\pi(x)=\pi(y)$ Since $\pi(y)$ was picked arbitrarily from $W$, then by the definition of $\mathfrak{M}(\mathcal{B})$ we obtain $\mathfrak{M}(\mathcal{B}), \pi(x) \Vdash \neg \mathrm{D} X$.

$\psi=\diamond^{-} \chi$. This case is handled analogously to the case $\psi=\diamond \chi$.

$\varphi=\psi \wedge \chi$. By application of $(\wedge)$ to $v_{1}(\psi \wedge \chi, x)$, we have $v_{1}(\psi, x) \in \mathcal{B}$ and $v_{1}(x, x) \in \mathcal{B}$. By the inductive hypothesis we have $\mathfrak{M}(\mathcal{B}), \pi(x) \Vdash \psi$ and $\mathfrak{M}(\mathcal{B}), \pi(x) \Vdash x$ and, thus, $\mathfrak{M}(\mathcal{B}), \pi(x) \Vdash \psi \wedge \chi$.

$\varphi=\diamond \psi$. Since the rule $(\diamond)$ is applied to $v_{1}(\diamond \psi, x)$ in $\mathcal{B}$ we have $R(x, f(\psi, x)) \in \mathcal{B}$ and $v_{1}(\psi, f(\diamond \psi, x)) \in \mathcal{B}$. By construction of $\mathbf{R}$ we conclude that $\langle\pi(x), \pi(f(\psi, x))\rangle \in \mathbf{R}$. Furthermore, by the inductive hypothesis, $\mathfrak{M}(\mathcal{B}), \pi(f(\psi, x)) \Vdash \psi$. Hence, $\mathfrak{M}(\mathcal{B}), \pi(x) \Vdash \diamond \psi$ by definition of truth relation.

$\varphi=@_{i} \psi$. We have that $v_{1}\left(@_{i} \psi, x\right) \in \mathcal{B}$. $\mathcal{B}$ is open and fully-expanded, so (@) has been applied. Therefore $v_{1}\left(\psi, v_{0}(i)\right) \in \mathcal{B}$. By the inductive hypothesis, $\mathfrak{M}, \pi\left(v_{0}(i)\right) \Vdash \psi$. By the definition of $\Vdash$ we obtain that $\mathfrak{M}, \pi(x) \Vdash @_{i} \psi$.

$\varphi=\mathrm{E} \psi$. Since $\mathcal{B}$ is open and fully expanded, the $(\mathrm{E})$-rule has been applied to $v_{1}(E \psi, x)$, once it occurred on $\mathcal{B}$. Therefore, it follows that $v_{1}(\psi, f(\mathrm{E} \psi, x)) \in \mathcal{B}$. By the inductive hypothesis, we obtain that $\mathfrak{M}(\mathcal{B}), \pi(f(\mathrm{E} \psi, x)) \Vdash \psi$, and by the definition of $\Vdash$ we have $\mathfrak{M}(\mathcal{B}), \pi(x) \Vdash \mathrm{E} \psi$.

$\varphi=\mathrm{D} \psi$. Since $\mathcal{B}$ is open and fully expanded, the (D)-rule has been applied to $v_{1}(E \psi, x)$, once it occurred on $\mathcal{B}$. Therefore, it follows that $v_{1}(\psi, f(D \psi, x)) \in \mathcal{B}$ and $x \neq f(D \psi, x) \in \mathcal{B}$. Since $\mathcal{B}$ is open and fully expanded, $x \approx f(\mathrm{D} \psi, x)$ could not have appeared on $\mathcal{B}$, thus it is not the case that $x \sim_{\mathcal{B}} f(D \psi, x)$ and by the definition of $\pi, \pi(x) \neq \pi(f(\mathrm{D} \psi, x)$. By the inductive hypothesis, we obtain that $\mathfrak{M}(\mathcal{B}), \pi(f(D \psi, x)) \Vdash \psi$ and by the definition of $\Vdash$ we get $\mathfrak{M}(\mathcal{B}), \pi(x) \Vdash \mathrm{D} \psi$.

$\varphi=\diamond^{-} \psi$. This case is handled analogously to the case $\varphi=\diamond \psi$. 
If we consider some particular frame classes, we also need to verify whether a model resulting from a branch belongs to this class. This, however, is merely a matter of routine and follows almost directly from the construction of $\mathfrak{M}(\mathcal{B})$.

THEOREM 7.3. $\mathcal{T}_{\mathcal{H}_{b-f}}^{p}$ is a constructively complete tableau calculus for $\mathcal{H}_{b-f}$

Proof. Follows from the construction of $\mathfrak{M}(\mathcal{B})$ and Lemma 7.2.

\subsection{RULE REFINEMENT OF $\mathcal{T}_{\mathcal{H}_{\text {B-F }}^{\text {P }}}$}

In [84], cases are presented where the rules of synthesised tableau calculi can be refined. Sometimes we can refine a calculus by decreasing the branching factor of particular rules, ipso facto reducing the size of a tableau. In order to do that, we turn some conclusions of a branching rule into its premises and invert negation in front of them. More precisely, let $\beta$ be a tableau rule $X_{0} / X_{1}|\cdots| X_{n}$ and $X_{j}=\left\{X_{1}, \ldots, X_{m}\right\}$. Refinements of the rule $\beta$ with respect to its $j$-th branch are the rules $\beta_{k}^{j}$ for $k=1, \ldots, m$ defined by

$$
\beta_{k}^{j}=\frac{X_{0,} \sim X_{k}}{X_{1}|\cdots| X_{j-1}\left|X_{j+1}\right| \cdots \mid X_{n}} .
$$

Here, for any formula $\varphi, \sim \varphi=\psi$ if $\varphi=\neg \psi$, and $\sim \varphi=\neg \varphi$ otherwise. A refinement $\mathcal{T}^{R}$ of a given calculus $\mathcal{T}$ is obtained by replacing a rule $\beta$ with its refinements $\beta_{1}^{j}, \ldots, \beta_{m}^{j}$.

Notwithstanding the conceptual simplicity of the foregoing method, not every branching rule can be refined without loosing completeness for the whole calculus. Furthermore, it might occur that even the same branching rule behaves differently in different tableau calculi allowing for refinement in one case but not in another. It emerges in the case of the rule $(\neg D)$, as described below.

It is straightforward that refinements of a rule are derivable in any given calculus. Hence, every derivation step in the refined calculus can be simulated in the original calculus. To obtain the converse and, thus, equivalence of the refined and original calculi we scrutinise the notion of admissibility of the original rule in the refined calculus. Hence, we 
need to provide the condition that would help to decide whether a rule $\beta$ is admissible in a calculus $\mathcal{T}^{R}$. In [84] we can find such a condition: THEOREM 7.4 ([84]). Let $\mathcal{T}$ be a tableau calculus. Let $\beta$ be the rule of the form:

$$
\frac{X_{0}}{X_{1}|\cdots| X_{n}}
$$

and let $\mathcal{T}^{R}$ be a refined version of $\mathcal{T}$, including the rule $\beta^{R}$ of the form:

$$
\frac{X_{0}, \neg X_{1}}{X_{2}|\cdots| X_{n}} \text {. }
$$

Suppose that $\mathcal{B}^{R}$ is an arbitrary open and fully-expanded branch in a $\mathcal{T}^{R}$-tableau. Let $F=\left\{\varphi_{1}, \ldots, \varphi_{l}\right\}$ be a set of all formulas from $\mathcal{B}^{R}$ reflected in $\mathfrak{M}\left(\mathcal{B}^{R}\right)$. Then the refined rule $\beta^{R}$ is admissible (i.e. the resulting calculus $\mathcal{T}^{R}$ is still complete) if the following condition is satisfied:

$$
\begin{aligned}
& \text { If } X_{0}\left(\varphi_{i_{1}}, \ldots, \varphi_{i_{k}}\right) \in \mathcal{B}^{R} \\
& \text { then } \mathfrak{M}\left(\mathcal{B}^{R}\right) \models X_{m}\left(\varphi_{i_{1}}, \ldots, \varphi_{i_{k}}\right), \text { for some } m \in\{1, \ldots, n\},
\end{aligned}
$$

where $X_{i}\left(\varphi_{j_{1}}, \ldots, \varphi_{j_{l}}\right)$ is an instantiation of a formula $X_{i}$, involving formulas $\varphi_{j_{1}}, \ldots, \varphi_{j l}$.

In particular, the $(t)$ condition says that any hypothetical application of the rule $\beta$ is redundant on the branch $\mathcal{B}$ of the refined calculus. As a corollary, we obtain the following statement.

THEOREM 7.5 ([84]). If $\mathcal{T}$ is constructively complete and the $(t)$ condition holds for every open and fully expanded branch in a refined $\mathcal{T}$-tableau, then the refined $\mathcal{T}$ is constructively complete.

In $\mathcal{T}_{\mathcal{H}_{\text {b-f }}}^{\mathrm{p}}$ two branching rules are refinable by decreasing their branching factor: the $(\neg \diamond)$ rule and the $(\neg D)$ rule (as shown in Fig. 14).

Proposition 7.6. Condition $(t)$ is satisfied in $\mathcal{T}_{\mathcal{H}_{\text {b-f }}}^{\mathrm{p}}$ for the rules $(\neg \diamond)$ and $(\neg \mathrm{D})$ from Fig. 14.

Proof. The case of $(\neg \diamond)$ can be proven in the following way. Let $\varphi$ be an arbitrary formula occurred in $\mathcal{B}$ and reflected in $\mathfrak{M}(\mathcal{B})$. Assume $\neg v(\nabla \varphi, x) \in \mathcal{B}$ and a domain term $y$ is occurred in $\mathcal{B}$. We must prove 
Refined $(\neg \diamond)$-rule:

$$
(\neg \diamond) \frac{\neg v_{1}(\nabla \varphi, x), y \approx y}{\neg R(x, y) \mid \neg v_{1}(\varphi, y)} \quad \rightsquigarrow \quad(\neg \diamond)^{R} \frac{\neg v_{1}(\nabla \varphi, x), R(x, y)}{\neg v_{1}(\varphi, y)}
$$

Refined $(\neg \mathrm{D})$ :

$$
(\neg \mathrm{D}) \frac{\neg v_{1}(\mathrm{D} \varphi, x), y \approx y}{\neg v_{1}(\varphi, y) \mid x \approx y} \quad \rightsquigarrow \quad(\neg \mathrm{D})^{R} \frac{\neg v_{1}(\mathrm{D} \varphi, x), x \neq y}{v_{1}(\varphi, y)}
$$

Figure 14: Refined rules of $\mathcal{T}_{\mathcal{H}_{\text {b-f }}^{\mathrm{p}}}^{\mathrm{p}}$

that either $\langle\pi(x), \pi(y)\rangle \in \mathrm{R}$ does not hold in $\mathfrak{M}(\mathcal{B})$ or $\mathfrak{M}(\mathcal{B}), \pi(y) \|$ $\varphi$. Suppose $\langle\pi(x), \pi(y)\rangle \in \mathrm{R}$ holds. By construction of $\mathfrak{M}(\mathcal{B})$ it means that $R(z, v), z \approx x, v \approx y$ must have occurred in $\mathcal{B}$. Thus, by the equality rules $R(x, y) \in \mathcal{B}$ and, consequently, the rule $(\neg \diamond)^{R}$ has been applied in $\mathcal{B}$. Therefore, $\neg v_{1}(\varphi, y) \in \mathcal{B}$. Because $\varphi$ is reflected in $\mathfrak{M}(\mathcal{B})$ this means that $\mathfrak{M}(\mathcal{B}), \pi(y) \| \varphi$.

Obviously, the same holds for $\diamond^{-}$.

For the case of $(\neg D)$ we allow ourselves an extensive remark below.

REMARK 7.1. What is interesting about tableau negative rules defined for $D$ is that in the context of ordinary modal logic (i.e. with no nominals) they are quite complicated and lead to multiple branching dependent on the number of prefixes already occurring on a branch (see [29] or [34]). In hybrid logics, the possibility of expressing inequality of nominals simplifies matters greatly for the rule (D). But still a suitable rule for negated $D$ is branching in most systems (e.g. [70, 42, 57]).

In [16], Blackburn and Bolander noticed that the $(\neg D)$ rule in the form present in our calculus breaks the completeness of the whole calculus but they did not explain why the branching rule does not violate the completeness of the calculus, whereas the version without branching conclusions does. In [84], Schmidt and Tishkovsky introduced the $(t)$ condition which we presented in the foregoing part of the section. In most modal and description logics $(t)$ holds for $(\neg \diamond)$ but, as it turns out, it fails for $(\neg D)$. Usually, to ma]ke such refinements 
1.

2.

3.

4.

5.

$$
\neg v_{1}(\mathrm{D} \varphi, x)
$$$$
y \approx y
$$

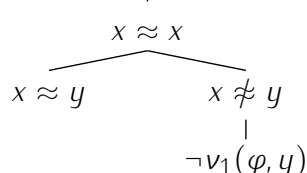

assumption

assumption

$$
(\approx): 1
$$

(ub): 2,3

$(\neg \mathrm{D})^{R}: 1,4$

Figure 15: Derivation of the initial rule $(\neg D)$ using the refined rule $(\neg D)$ and (ub).

possible without breaking completeness, we need the analytical cut rule of the form:

$$
\frac{x \approx x}{v_{1}(\varphi, x) \mid \neg v_{1}(\varphi, x)}, \varphi \in \operatorname{Sub}(\text { input) },
$$

which significantly increases the branching factor of the whole calculus. However, it appears that in the case of $(\neg D)$ rule we only need some restricted form of the analytical cut for expressions of the domain sort:

$$
\frac{x \approx x, y \approx y}{x \approx y \mid x \neq y}
$$

which is present in $\mathcal{T}_{\mathcal{H}_{\text {b-f }}}^{\mathrm{p}}$ under the name of (ub). It turns out that thanks to the tool that was introduced for ensuring termination, we obtain the refined $(\neg \mathrm{D})^{R}$ rule for free! Taking the refined rule $(\neg \mathrm{D})^{R}$ and (ub) as primitive, we can derive the initial rule $(\neg D)$ (see Fig. 15).

Refining any of the remaining branching rules turns $\mathcal{T}_{\mathcal{H}_{\text {b-f }}}^{\mathrm{p}}$ into an incomplete calculus. For instance, the rule $(\neg \wedge)$ is not refinable. An example showing that $(\neg \wedge)$ cannot be refined to

$$
\frac{\neg v_{1}(\varphi \wedge \psi, x), v_{1}(\varphi, x)}{\neg v_{1}(\psi, x)}
$$

is the formula $v_{1}(\neg(\neg p \wedge \neg q), x)$, where $p$ and $q$ are propositional variables. Obviously, $\neg(\neg p \wedge \neg q)$ is logically equivalent to $p \vee q$ and thus satisfiable. In the refined tableau calculus only one rule is applicable, namely $(\neg)$, resulting in $\neg v_{1}(\neg p \wedge \neg q, x)$. The model constructed from 
a branch is $\mathfrak{M}(\mathcal{B})=\langle\mathrm{W}, \mathrm{R}, \mathrm{V}\rangle$ where $\mathrm{W}=\{\{x\}\}, \mathbf{R}=\varnothing, \mathrm{V}(p)=\varnothing$, $\mathrm{V}(q)=\varnothing$. It therefore does not satisfy the input.

As indicated before, all such failures could be neutralized by introducing an analytical cut rule which is the tableau-counterpart to the law of excluded middle.

\subsection{INTERNALIZED CALCULUS FOR $\mathcal{H}_{\mathrm{B}-\mathrm{F}}$}

In the present section we establish an internalized calculus for the logic $\mathcal{H}_{\text {b-f. }}$. We call it $\mathcal{T}_{\mathcal{H}_{\text {b-f }}}^{\mathrm{i}}$. Since there are some significant differences between the rules of $\mathcal{T}_{\mathcal{H}_{\text {b-f }}}^{p}$ and of $\mathcal{T}_{\mathcal{H}_{\text {b-f }}}^{\mathrm{i}}$ especially in the part concerning equality reasoning, we conduct an independent completeness proof for $\mathcal{T}_{\mathcal{H}_{\text {b-f }}}^{\mathrm{i}}$ (for the part not covered in [16] and [19]).

\subsubsection{Encoding domain sort expressions}

In [10], Blackburn made an observation that the language of hybrid logic with@operators is sufficiently rich to express its semantics within itself. As we indicated in Section 4.3 of Chapter 4, there are three types of the domain expressions: satisfaction statements ( $\mathfrak{M}, w \Vdash$ $\varphi)$, accessibility statements $(w R v)$ and equality statements $(w=v)$. Hybrid equivalents of the foregoing expressions are shown below:

$$
\begin{aligned}
& \mathcal{H}(\mathfrak{M}, w \Vdash \varphi)=@_{i_{w}} \varphi, \quad \mathcal{H}(\mathfrak{M}, w \mathbb{H} \varphi)=@_{i_{w}} \neg \varphi \text {, } \\
& \mathcal{H}(R(w, v))=@_{i_{w}} \nabla j_{v}, \quad \mathcal{H}(\neg R(w, v))=@_{i_{w}} \neg \nabla j_{v}, \\
& \mathcal{H}(w=v)=@_{i_{w}} j_{v}, \quad \mathcal{H}(w \neq v)=@_{i_{x}} \neg j_{y} \text {. }
\end{aligned}
$$

Both $\mathrm{E}$ and D operators allow mimicking @ operators: @i $\varphi:=\mathrm{E}(i \wedge$ $\varphi)$ and @i $\varphi:=(i \wedge \varphi) \vee \mathrm{D}(i \wedge \varphi)$. Therefore, in the calculus we use the notation $i: \varphi$, rather than @ $i \varphi$, to keep its universal character. This colon notation will stand for one of the foregoing expressions, depending on a considered logic, except for the fact that whenever a logic includes@operators, $i: \varphi$ means @i $\varphi$. 


\subsubsection{Tableau calculus $\mathcal{T}_{\mathcal{H}_{b-f}}^{i}$}

Figure 16 presents the rules of the tableau calculus $\mathcal{T}_{\mathcal{H}^{\mathrm{b}-\mathrm{f}}}^{\mathrm{i}}$ for the logic $\mathcal{H}_{\text {b-f. }}$

The rule $(\neg)$ is only applied to negated nominals that appear in the scope of a label. The aim of introducing this rule to the calculus is to make subjected negated nominals to equality reasoning, or, more generally, not to distinguish between nominals that appear on a branch as labels and those appearing as formulas, i.e. we want to fully respect their hybrid nature. Other Boolean rules again are straightforward and require no additional comments. $(\diamond),(E),(D)$ and $\left(\diamond^{-}\right)$are rules introducing new labels, which was marked as the side-condition for them ${ }^{4}$. In the case of $(\neg E)$ and $(\neg D)$, the standard side-condition of a former occurrence of a label on a branch was replaced by introducing an explicit premiss stating that a particular nominal has appeared as a label on a branch. The rule (ref:) is a reflexivity rule that introduces to a branch an explicit information that a nominal occurred as a label within a branch. (sub) expresses the substitutability of two nominals as labels, provided that one of them is labelled by the other. As we can see the total number of rules for equality reasoning shrunk comparing to the calculus $\mathcal{T}_{\mathcal{H}_{\mathrm{b}-\mathrm{f}}}^{\mathrm{p}}$. In the forthcoming part of this section, we show that it suffices to ensure that equality reasoning follows properly. The $(\perp)$ rule is self-evident. The rules for frame conditions are standard. In some other internalized calculi for hybrid logics, like in [10], an additional rule for equality reasoning is introduced, namely:

$$
\text { (bridge) } \frac{i: \diamond j, j: k}{i: \diamond k}
$$

However, in our method of constructing a model out of a fully-expanded open branch this rule becomes superfluous and it is successfully covered by $(\neg)$, (ref:) and (sub).

As indicated in Section 7.2.2, (ub) rule is a variant of the analytical cut rule restricted to nominals only, which was sucessfully applied to ensure termination of the calculus. Simultaneously, it allows turning $(\neg \mathrm{D})$ into a non-branching rule.

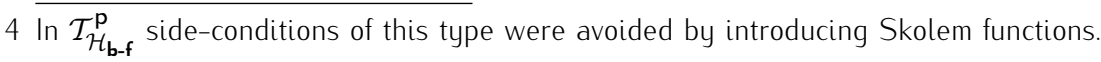


Rules for connectives:
(ᄀ) $\frac{i: \neg j}{j: j}$
$(\neg \neg) \frac{i: \neg \neg \varphi}{i: \varphi}$
$(\wedge) \frac{i: \varphi \wedge \psi}{i: \varphi, i: \psi}$
$(\neg \wedge) \frac{i: \neg(\varphi \wedge \psi)}{i: \neg \varphi \mid i: \neg \psi}$
$(\diamond)^{*} \frac{i: \diamond \varphi}{i: \diamond j, j: \varphi} \quad(\neg \diamond) \frac{i: \neg \nabla \varphi, i: \diamond j}{j: \neg \varphi}$
(@) $\frac{i: @ j \varphi}{j: \varphi}$
(ᄀ@) $\frac{i: \neg @ j \varphi}{j: \neg \varphi}$
$(\mathrm{E})^{*} \frac{i: \mathrm{E} \varphi}{j: \varphi}$
$(\neg \mathrm{E}) \frac{i: \neg \mathrm{E} \varphi, j: j}{j: \neg \varphi}$
$(\mathrm{D})^{*} \frac{i: \mathrm{D} \varphi}{i: \neg j, j: \varphi}$
$(\neg \mathrm{D}) \frac{i: \neg \mathrm{D} \varphi, i: \neg j}{j: \neg \varphi}$
$\left(\diamond^{-}\right)^{*} \frac{i: \diamond^{-} \varphi}{j: \diamond i, j: \varphi}$
$\left(\neg \diamond^{-}\right) \frac{i: \neg \nabla^{-} \varphi, j: \diamond i}{j: \neg \varphi}$

Rules for equality:

$$
\text { (ref:) } \frac{i: \varphi}{i: i} \quad \text { (sub) } \frac{i: j, i: \varphi}{j: \varphi}
$$

Closure rule and unrestricted blocking rule:
$(\perp) \frac{i: \varphi, i: \neg \varphi}{\perp}$
(ub) $\frac{i: i, j: j}{i: j \mid i: \neg j}$

Rules for frame conditions:

$$
\begin{aligned}
\text { (ref) } \frac{i: i}{i: \diamond i} \quad(\text { sym }) \frac{i: \diamond j}{j: \diamond i} & (\text { tran }) \frac{i: \diamond j, j: \diamond k}{i: \diamond k} \\
\left(\text { Eucl) } \frac{i: \diamond j, i: \diamond k}{j: \diamond k}\right. & (\text { ser })^{*} \frac{i: i}{i: \diamond j}
\end{aligned}
$$

* Nominals in the conclusions are fresh on the branch.

$$
\text { Figure 16: Rules for the calculus } \mathcal{T}_{\mathcal{H}_{\text {b-f }}}^{\mathrm{i}}
$$




\subsubsection{Decision procedure based on $\mathcal{T}_{\mathcal{H}_{b-f}}^{i}$}

Before we provide a proper method of constructing a tableau, let's recall several preliminary definitions.

DEFINITION 7.2. Let $\operatorname{NOM}(\mathcal{B})$ be a set of nominals occurring as labels on a fully expanded branch $\mathcal{B}$ of a $\mathcal{T}_{\mathcal{H}_{\text {b-f }}}$ tableau for a given input formula. Like in the case of $\mathcal{T}_{\mathcal{H}_{\text {b-f }}}^{\text {p }}$ we introduce the $\sim_{\mathcal{B}}$ relation over $\operatorname{NOM}(\mathcal{B})$ which we define in the following way:

$$
i \sim \mathcal{B} j \text { iff } i: j \in \mathcal{B} .
$$

Proposition 7.7. $\sim_{\mathcal{B}}$ is the equivalence relation.

Proof. Reflexivity is ensured by the (ref:) and the $(\neg)$ rule. For symmetry assume that $i: j$ is on $\mathcal{B}$. By (ref:) we obtain $i$ : $i$ and after applying (sub) to these two premises we obtain $j: i$. For transitivity suppose that $i: j$ and $j: k$ are on $\mathcal{B}$. By symmetry we have that $j: i$ is also on $\mathcal{B}$. We therefore take $j: i$ and $j: k$ as premises of (sub) and obtain $i: k$.

DEFINITION 7.3. A rule of the $\mathcal{T}_{\mathcal{H}_{\text {b-f }}}$ is applied eagerly in a tableau if, and only if whenever it is applicable, it is applied.

DEFINITION 7.4. Let $\prec_{\mathcal{B}}$ be an ordering on $\operatorname{NOM}(\mathcal{B})$ defined as follows:

$$
i \prec_{\mathcal{B}} j \text { iff } i: i \text { occurred on } \mathcal{B} \text { earlier than } j: j \text {. }
$$

Note that $\prec_{\mathcal{B}}$ is well-founded and linear since no rule introduces more than one labelling nominal as a conclusion.

Definition 7.5. To each $\mathcal{T}_{\mathcal{H}_{\text {b-f }}}$ rule we affix the priority number. It indicates what the order of application of particular rules should be. The lower the number is, the sooner the rule should be applied. We have: (ub): $1,($ ref $),(\neg),($ sub $),(\neg \neg),(\wedge),(\neg \wedge),(\neg \diamond),(\neg E),(\neg D)$, $\left(\neg \diamond^{-}\right): 2,(\diamond),(\mathrm{E}),(\mathrm{D}),\left(\diamond^{-}\right): 3$.

Now we are ready to provide the tableau construction algorithm. As usual, we do it inductively.

DEFINITION 7.6 (Tableau construction algorithm). We define the algorithm inductively. 
basic step: For a given input formula $\varphi$ put $i: \varphi$ at the initial node. $i$ is a nominal not occurring in $\varphi$.

inductive step: Suppose that you performed $n$ steps of a derivation. In the $n+1$-th step apply the rules of $\mathcal{T}_{\mathcal{H}_{\text {b-f }}}$ eagerly respecting the priority ordering given in Definition 7.5 and fulfilling the following conditions:

(c1) if the application of a rule results in a formula that is already present on a branch, do not perform this application,

(c2) rules of priority 3 can only by applied to labels that are the least elements (with respect to $\prec_{\mathcal{B}}$ ) of the equivalence class (with respect to $\sim \mathcal{B})$,

(c3) the $(\diamond)$ must not be applied to formulas of the form $i: \diamond j$. We call them the accessibility formulas,

(c4) apply the $(\perp)$ rule whenever it is possible.

If after the $n+1$-th step of derivation:

(a) all tableau branches are closed, stop and return: unsatisfiable,

(b) there is an open branch in a tableau and no further rules are applicable (respecting conditions (c1)-(c4)), stop and return: satisfiable,

(c) there is an open branch in a tableau and further rules are applicable (respecting conditions $(\mathrm{c} 1)-(\mathrm{c} 4)$ ), proceed to the $n+2$-th step.

We will explain a role that the (ub) rule plays in ensuring termination more carefully in Section 7.6.

\subsubsection{Soundness and Completeness of $\mathcal{T}_{\mathcal{H}_{b-f}}^{i}$}

In the current section we state and prove soundness and completeness of the foregoing calculus. For the definitions of soundness and completeness of a tableau calculus, we refer the reader to Section 7.2.3.

As usual, demonstrating soundness amounts to proving that particular rules preserve satisfiability. For the completeness, we take the contrapositive of the condition given at the beginning of Section 7.2.3 and demonstrate that if there exists an open, fully expanded branch $\mathcal{B}$ in a tableau for $\varphi$ then there exists a model for $\varphi$. 
THEOREM 7.8. $\mathcal{T}_{\mathcal{H}_{b-f}}^{i}$ is sound.

Proof. By easy verification of all the rules.

Suppose that $\mathcal{B}$ is an open, fully expanded branch in a $\mathcal{T}_{\mathcal{H}_{\mathrm{b}-\mathrm{f}}}^{\mathrm{i}}$ tableau for $\varphi$. We define a model $\mathfrak{M}(\mathcal{B})=\langle W, R, V\rangle$ derived from $\mathcal{B}$ in the following way:

$\mathrm{W}=\left\{[i]_{\sim \mathcal{B}} \mid i: i \in \mathcal{B}\right\}$,

$\mathrm{R}=\left\{\left\langle[i]_{\sim_{\mathcal{B}}}[j]_{\approx_{\mathcal{B}}}\right\rangle \mid i: \diamond j: \in \mathcal{B}\right\}$,

$\mathrm{V}=\left\{\left(i,[i]_{\sim_{\mathcal{B}}}\right) \mid i: i \in \mathcal{B}\right\} \cup\{(p, U) \mid p \in$ PROP, $p$ occurred in $\mathcal{B}$ and $\left.U=\left\{[i]_{\sim_{\mathcal{B}}} \mid i: p \in \mathcal{B}\right\}\right\}$.

Lemma 7.9. Suppose that $\mathcal{B}$ is an open, fully expanded branch in a $\mathcal{T}_{\mathcal{H}_{\text {b-f }}}^{\mathrm{i}}$ tableau for $\varphi$. Then if $i: \psi \in \mathcal{B}$ then $\mathfrak{M}(\mathcal{B}),[i]_{\sim_{\mathcal{B}}}=\psi$.

Proof. By induction on the complexity of $\psi$. Since all cases save $\psi=\mathrm{D} X$ and $\psi=\neg \mathrm{D} X$ are covered by proofs given in [16] and [19] (in a little more complicated form involving the notion of urfather instead of the ordinary equivalence relation $\approx$ ), we only consider missing cases.

$\varphi=\mathrm{D} \psi$. We have $i: \mathrm{D} \psi \in \mathcal{B}$. After applying (D) we obtain $i: \neg j \in \mathcal{B}$ and $j: \psi \in \mathcal{B}$. By the inductive hypothesis we have that $\mathfrak{M}(\mathcal{B}),[j]_{\sim_{\mathcal{B}}} \Vdash \psi$. It suffices to show that $[i]_{\sim_{\mathcal{B}}}$ and $[j]_{\sim_{\mathcal{B}}}$ are distinct. Suppose that they are the same equivalence class. But then, by Definition 7.2, $i: j \in \mathcal{B}$, which contradicts the fact that $\mathcal{B}$ is open.

$\varphi=\neg \mathrm{D} \psi$. We have $i: \neg \mathrm{D} \psi \in \mathcal{B}$. Let $\operatorname{Nom}(\mathcal{B})$ be the set of labels that appeared on $\mathcal{B}$. Since the (ub) rule is applied eagerly, for each label $j_{k} \in \operatorname{NOM}(\mathcal{B})$ either $i: j_{k} \in \mathcal{B}$ or $i: \neg j_{k} \in \mathcal{B}$. If for all labels $j_{k}$ from $\operatorname{Nom}(\mathcal{B})$ we have $i: j_{k} \in \mathcal{B}$, it means that $\mathrm{W}=\left\{[i]_{\sim_{\mathcal{B}}}\right\}$ and therefore $\mathfrak{M}(\mathcal{B}),[i]_{\sim_{\mathcal{B}}} \Vdash \neg \mathrm{D} \psi$ trivially holds. Suppose that there exists a label $j_{l} \in \mathcal{L}$ such that $i: \neg j_{l} \in \mathcal{B}$. Then, after applying $(\neg \mathrm{D})$ to $i: \neg \mathrm{D} \psi$ and $i: \neg j l$, we obtain that $j_{l}: \neg \psi \in \mathcal{B}$. By the inductive hypothesis, $\mathfrak{M}(\mathcal{B}),[j]_{\sim_{\mathcal{B}}} \Vdash \neg \psi$ and $[i]_{\sim_{\mathcal{B}}} \neq\left[j_{k}\right]_{\sim_{\mathcal{B}}}$. Since $j l$ was picked arbitrarily, we obtain the conclusion.

THEOREM 7.10. $\mathcal{T}_{\mathcal{H}_{\text {b-f }}}^{i}$ is complete.

Proof. By the definition of the completeness and Lemma 7.9. 


$$
\begin{aligned}
& \text { (ax) } \Gamma \Rightarrow \Delta \text {, where } \Gamma \cap \Delta \neq \varnothing \\
& (\neg \Rightarrow) \frac{\Gamma \Rightarrow \Delta, i: \varphi}{i: \neg \varphi, \Gamma \Rightarrow \Delta} \quad(\Rightarrow \neg) \frac{i: \varphi, \Gamma \Rightarrow \Delta}{\Gamma \Rightarrow \Delta, i: \neg \varphi} \\
& (\wedge \Rightarrow) \frac{i: \varphi, i: \psi, \Gamma \Rightarrow \Delta}{i: \varphi \wedge \psi, \Gamma \Rightarrow \Delta} \quad(\Rightarrow \wedge) \frac{\Gamma \Rightarrow \Delta, i: \varphi \mid \Gamma \Rightarrow \Delta, i: \psi}{\Gamma \Rightarrow \Delta, i: \varphi \wedge \psi} \\
& (@) \frac{j: \varphi, \Gamma \Rightarrow \Delta}{i: @_{j} \varphi, \Gamma \Rightarrow \Delta} \quad(\Rightarrow @) \frac{\Gamma \Rightarrow \Delta, j: \varphi}{\Gamma \Rightarrow \Delta, i: @ j \varphi} \\
& (\text { ref })^{2} \frac{i: i, \Gamma \Rightarrow \Delta}{\Gamma \Rightarrow \Delta} \quad \text { (sub) } \frac{j: \varphi, i: j, i: \varphi, \Gamma \Rightarrow \Delta}{i: j, i: \varphi, \Gamma \Rightarrow \Delta} \\
& (\diamond \Rightarrow)^{1} \frac{i: \diamond j, j: \varphi, \Gamma \Rightarrow \Delta}{i: \diamond \varphi, \Gamma \Rightarrow \Delta} \quad(\Rightarrow \diamond) \frac{i: \diamond j, \Gamma \Rightarrow \Delta, i: \diamond \varphi, j: \varphi}{i: \diamond j, \Gamma \Rightarrow \Delta, i: \diamond \varphi} \\
& \left(\diamond^{-} \Rightarrow\right)^{1} \frac{j: \diamond i, j: \varphi, \Gamma \Rightarrow \Delta}{i: \diamond^{-} \varphi, \Gamma \Rightarrow \Delta} \quad\left(\Rightarrow \diamond^{-}\right) \frac{j: \diamond i, \Gamma \Rightarrow \Delta, i: \diamond^{-} \varphi, j: \varphi}{j: \diamond i, \Gamma \Rightarrow \Delta, i: \diamond^{-} \varphi} \\
& (\mathrm{E} \Rightarrow)^{1} \frac{j: \varphi, \Gamma \Rightarrow \Delta}{i: \mathrm{E} \varphi, \Gamma \Rightarrow \Delta} \quad(\Rightarrow \mathrm{E}) \frac{j: j, \Gamma \Rightarrow \Delta, i: \mathrm{E} \varphi, j: \varphi}{j: j, \Gamma \Rightarrow \Delta, i: \mathrm{E} \varphi} \\
& (\mathrm{D} \Rightarrow)^{1} \frac{j: \varphi, \Gamma \Rightarrow \Delta, i: j}{i: \mathrm{D} \varphi, \Gamma \Rightarrow \Delta} \quad(\Rightarrow \mathrm{D}) \frac{\Gamma \Rightarrow \Delta, i: \mathrm{D} \varphi, i: j, j: \varphi}{\Gamma \Rightarrow \Delta, i: \mathrm{D} \varphi, i: j} \\
& 1 \text { where } j \text { does not occur in } \Gamma \cup \Delta \cup\{\varphi\} \text {. } \\
& 2 \text { provided that } i \text { occurs in } \Gamma \cup \Delta \text {. }
\end{aligned}
$$

Figure 17: Rules for the sequent calculus $\mathcal{S}_{\mathcal{H}_{\text {b-f }}}$.

\subsection{SEQUENT CALCULUS FOR $\mathcal{H}_{\mathrm{B}-\mathrm{F}}$}

Before we go to termination matters, we develop the sequent calculus corresponding strictly to the tableau calculus presented above. We use sequents built from finite sets of formulas which allow to avoid structural rules and to keep strong resemblance to the rules of the tableau calculus (see Fig. 17).

The special rule for unrestricted blocking has the form:

$$
\text { (ub) } \frac{i: i, j: j, \Gamma \Rightarrow \Delta, i: j \mid i: j, i: i, j: j, \Gamma \Rightarrow \Delta}{i: i, j: j, \Gamma \Rightarrow \Delta}
$$


The proof is defined in a standard way as a tree of sequents where each leaf is labelled with an instance of an axiom and all edges are obtained by means of specified rules.

REMARK 7.2. All the rules are in one-to-one correspondence to tableau rules, except two rules for negation. Here instead of one tableau rule $(\neg \neg)$ we have two rules; on the other hand, the effect of the tableau rule $(\neg)$ is covered by the more general formulation of the rule (ref). We explicitly introduced $j: j$ into antecedents of schemata of $(\Rightarrow E)$, $(\Rightarrow \mathrm{D})$ and (ub) in order to closely follow the formulation of $(\neg E)$, $(\neg D)$, (ub). Clearly, one can formulate this demand as a side condition for both rules and thus avoid their presence.

Remark 7.3. Duplication of main formulas in premises of (sub), $(\Rightarrow \mathrm{D}),(\Rightarrow \mathrm{E}),(\Rightarrow \diamond)$ and $\left(\Rightarrow \diamond^{-}\right)$(as well as $j: j$ in $(\Rightarrow \mathrm{D})$ and $(\Rightarrow \mathrm{E}))$ is necessary for keeping completeness due to lack of contraction and using sets (not multisets) of formulas in sequents.

Completeness of this calculus follows from the completeness result established for the tableau calculus, since one can easily rewrite each tableau proof as a proof in the sequent calculus specified above.

One can easily prove in the standard way (cf. e.g. Negri, von Plato [71]) that rules of weakening in antecedent and succedent are admissible in this calculus. Moreover, admissibility of (cut) may be proven constructively, in the similar way as for Myers' and Pattinson's sequent calculus in $[70]$ if we replace $(\Rightarrow D)$ with its two-premise counterpart corresponding to the unrefined negative tableau rule for $\mathrm{D}^{5}$ :

$$
\frac{j: j, \Gamma \Rightarrow \Delta, i: \mathrm{D} \varphi, j: \varphi \mid i: j, j: j, \Gamma \Rightarrow \Delta, i: \mathrm{D} \varphi}{j: j, \Gamma \Rightarrow \Delta, i: \mathrm{D} \varphi}
$$

In fact, from the standpoint of syntactical purity of the rules, normally required from sequent calculi, such a rule is better. Well-behaved rules

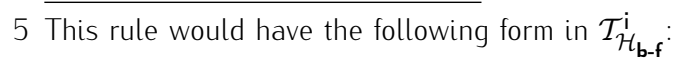

$$
(\neg \mathrm{D}) \frac{i: \neg \mathrm{D} \varphi, j: j}{j: \neg \varphi \mid i: j} .
$$


of sequent calculi should have only (one) occurrence of the main formula in conclusion-sequent and this requirement is not satisfied by our calculus since in some rules we have also explicit occurences of some additional formulas. In order to avoid this situation, one can delete occurences of $j: j$ in the antecedents of $(\Rightarrow \mathrm{E}),(\Rightarrow \mathrm{D})$ and $(\mathrm{ub})$ in favour of suitable side condition, and replace $(\Rightarrow \diamond)$ and $\left(\Rightarrow \diamond^{-}\right)$with two-premise variants of the form:

$$
\frac{\Gamma \Rightarrow \Delta, i: \diamond \varphi, i: \diamond j \mid \Gamma \Rightarrow \Delta, i: \diamond \varphi, j: \varphi}{\Gamma \Rightarrow \Delta, i: \diamond \varphi}
$$

In this way, we obtain a calculus with bigger branching factor but with rules which better fit the ordinary shape of sequent calculus' rules and with no need of (cut) (including (ub)). But from the standpoint of simpler proof-trees, the reduction of branching factor is essential and we can move in the other direction as well. One can introduce the full analytic (cut) as the only branching rule (which covers (ub) as a special case) and get rid with all other branching rules. In this case we additionaly replace $(\Rightarrow \wedge)$ with its one-premise equivalents:

$$
\frac{i: \varphi, \Gamma \Rightarrow \Delta, i: \psi}{i: \varphi, \Gamma \Rightarrow \Delta, i: \varphi \wedge \psi} \quad \text { or } \quad \frac{i: \psi, \Gamma \Rightarrow \Delta, i: \varphi}{i: \psi, \Gamma \Rightarrow \Delta, i: \varphi \wedge \psi}
$$

Such a system is a sequent calculus counterpart of Mondadori and D'Agostin0 [26] KE system. In such a system (ub), and generally, analytical (cut) is not eliminable but such a system still provides decision procedure, and moreover, the length of proofs is essentially smaller (see [26]).

\subsection{TERMINATION OF $\mathcal{T}_{\mathcal{H}_{\text {B-F }}}^{\text {I }}$}

In this section, a proof that a decision procedure designed on the basis of $\mathcal{T}_{\mathcal{H}_{\text {b-f }}}^{\mathrm{i}}$ terminates is conducted. However, as we realize soon, the slightly modified proof of this fact also covers an analogous case of the calculus $\mathcal{T}_{\mathcal{H}_{\text {b-f }}}^{\mathrm{p}}$ and possible decision procedures given rise by it.

Exploiting the (ub) rule and the conditions (c1)-(c4) we show that $\mathcal{T}_{\mathcal{H}_{\text {b-f }}}^{\mathrm{i}}$ is terminating for the logic $\mathcal{H}_{\mathrm{b}-\mathrm{f}}$, provided that it has the finite model property for a certain class of frames. 


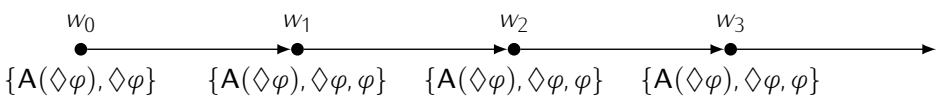

(a)

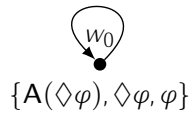

(b)

Figure 18: (a) and (b) present, respectively, an infinite and a finite (minimal) model for the formula $\mathrm{A}(\diamond \varphi)$. Both of them can be obtained from a tableau if the $(\mathrm{ub})$ rule is involved, since it allows merging worlds in an arbitrary way, provided that the consistency is preserved.

First, we make a remark that will be useful afterwards (cf. [83]).

REMARK 7.4. For each $[i]_{\sim_{\mathcal{B}}}$ the number of applications of the rules introducing a new label, namely $(\diamond),(E),(D),\left(\diamond^{-}\right)$to members of $[i]_{\sim_{\mathcal{B}}}$ is finite.

Proof. Indeed, if the (ub) is eagerly applied and the conditions (c2) and (c3) are fulfilled, it ensures that no superfluous application of $(\diamond),(E)$, (D), $\left(\diamond^{-}\right)$is performed, since they are only applied to one member of $[i]_{\sim_{\mathcal{B}}}$ and are not applied to accessibility formulas (otherwise it would lead to an infinite derivation that could not be subjected to blocking). Since the input formula $\varphi$ is assumed to be finite, therefore for each $i$ that occurred in $\mathcal{B}$, the number of $(\diamond),(E),(D),\left(\diamond^{-}\right)$applications to $[i]_{\sim_{\mathcal{B}}}$ is finite.

Corollary 7.11. For each $\mathcal{T}_{\mathcal{H}_{\mathrm{b}-\mathrm{f}}}^{\mathrm{i}}$ tableau branch $\mathcal{B}$ is finite iff $W$ of $\mathfrak{M}(\mathcal{B})$ is finite.

Now we are ready to state the lemma that is essential for termination of $\mathcal{T}_{\mathcal{H}_{\text {b-f }}}^{\mathrm{i}}$. However, before we do this, we explain informally how the (ub) rule works. Our tableau calculus by default handles all distinct nominals that were introduced to a branch as labelling distinct worlds. It leads to a situation where a satisfiable formula having a simple model generates an infinite tableau (see Fig. 18 ). The (ub) rule compares all labels that occurred on a branch and its left conclusion merges each 
pair unless it leads to the inconsistency. As a consequence, if a formula has a model $\mathfrak{M}$ of a certain cardinality, it will be reflected by a finite, fully expanded open branch of a $\mathcal{T}_{\mathcal{H}_{\text {b-f }}}$ tableau. The reason is that the left conclusion of the ( $u b)$ rule decreases the cardinality of a model whenever possible, so a model of the cardinality not-greater than the cardinality of $\mathfrak{M}$ will eventually be obtainable from one of the branches of a tableau. The formal argument is presented in the following lemma.

LEMMA 7.12. Suppose that a finite model $\mathfrak{N}=\langle\mathbf{U}, \mathbf{S}, \mathbf{Z}\rangle$ satisfies a formula $\varphi$. Then there exists an open branch $\mathcal{B}$ in a $\mathcal{T}_{\mathcal{H}_{\text {b-f }}}^{\mathrm{i}}$ tableau and $\mathfrak{M}(\mathcal{B})=\langle\mathrm{W}, \mathrm{R}, \mathrm{V}\rangle$ such that $\operatorname{Card}(\mathrm{W}) \leq \mathrm{Card}(\mathrm{U})$.

Proof. We proceed by induction on the number of steps in the derivation. During the derivation we construct a branch $\mathcal{B}$ in such a way that $\mathfrak{M}(\mathcal{B})$ is partially isomorphic to $\mathfrak{N}$ (cf. [83]).

basic step: $\varphi$ is satisfiable on $\mathfrak{N}$, so there must exist $\mathbf{w} \in U$ such that $\mathfrak{N}, \mathbf{w} \Vdash \varphi$. If also $\mathfrak{N}$, w $\Vdash i$ such that $i$ does not occur in $\varphi$, we put at the initial node of the derivation $i: \varphi$. If no such nominal holds in $\mathbf{w}$, we conservatively extend $\mathfrak{N}$ by adding fresh nominal $i$ to $\mathbf{w}$ and put at the initial node of the derivation $i: \varphi$.

inductive step: Application of each tableau rule should be considered as a separate case. Nevertheless, only five rules seem to be essential for this proof, namely $(\diamond),(E),(D),\left(\diamond^{-}\right)$and $(u b)$, i.e. rules that either introduce a new label to a branch or identify labels already present on a branch. We consider each of them.

$(\diamond)$. Suppose that a formula $\diamond \psi$ occurred at the $n$-th node of the derivation. It means that we associated the label $i$ of this node with a world in $U$ that satisfies $\diamond \psi$ and $i$. It follows that there must exist a world $\mathbf{v}$ such that $w S v$ and $\mathfrak{N}, v=\psi$. If $v$ does not satisfy any nominal $/$ that has not yet occurred on the branch either as a label or as a subformula, we conservatively extend $\mathfrak{N}$ by ascribing $l$ to $\mathbf{v}$. Applying $(\diamond)$ to $\diamond \psi$ we obtain $i: \diamond j$ and $j: \psi$. We put $l$ in place of $j$. (E). Suppose that a formula $E \psi$ occurred at the $n$-th node of the derivation. It means that we associated the label $i$ of this node with a world in $U$ that satisfies $\mathrm{E} \psi$ and $i$. Therefore, there exists a world $\mathrm{v}$ such that $\mathfrak{N}, \mathbf{v} \Vdash \psi$. If $\mathrm{v}$ does not satisfy any nominal $/$ that has 
not yet occurred on a branch either as a label or as a subformula, we conservatively extend $\mathfrak{N}$ by ascribing / to $v$. Applying (E) to $E \psi$ we obtain $j: \psi$. We put $l$ in place of $j$.

(D). Suppose that a formula $\mathrm{D} \psi$ occurred at the $n$-th node of the derivation. It means that we affixed the label $i$ of this node to a world in $U$ that satisfies $\mathrm{D} \psi$ and $i$. Therefore, there exists a world $\mathrm{v}$ such that $\mathfrak{N}, \mathbf{v} \Vdash \psi \wedge \neg i$. If $\mathrm{v}$ does not satisfy any nominal $/$ that has not yet occurred on a branch either as a label or as a subformula, we conservatively extend $\mathfrak{N}$ by ascribing / to v. Applying (D) to $\mathrm{D} \psi$ we obtain $j: \neg i$ and $j: \psi$. We put $l$ in place of $j$.

$\left(\diamond^{-}\right)$. Suppose that a formula $\diamond^{-} \psi$ occurred at the $n$-th node of the derivation. It means that we associated the label $i$ of this node with a world in $U$ that satisfies $\nabla^{-} \psi$ and $i$. It follows that there must exist a world $\mathbf{v}$ such that $\mathrm{vSw}$ and $\mathfrak{N}, \boldsymbol{v} \Vdash \psi$. If $\mathbf{v}$ does not satisfy any nominal $l$ that has not yet occurred on the branch either as a label or as a subformula, we conservatively extend $\mathfrak{N}$ by ascribing $l$ to $\mathbf{v}$. Applying $(\diamond)^{-}$to $\diamond^{-} \psi$ we obtain $j: \diamond i$ and $j: \psi$. We put $l$ in place of $j$.

( $u b$ ). Suppose that during the derivation two labels $i$ and $j$ have been introduced to $\mathcal{B}$. By the inductive hypothesis we mapped these labels to worlds $w$ and $v$ of (the conservative extension of) $U$. Either the world $\mathrm{w}$ satisfies $i \wedge j$ (which would mean that $\mathbf{w}$ and $\mathbf{v}$ are the same world) or it satisfies $i \wedge \neg j$ (which indicates that $\mathbf{w}$ and $\mathbf{v}$ are distinct). If the former is the case, we pick the left conclusion of ( $u b)$ and add it to $\mathcal{B}$, if the latter is the case, we choose the right conclusion of ( $u b$ ) and add it to $\mathcal{B}$.

Since $\mathcal{B}$ is open, we can construct a model $\mathfrak{M}(\mathcal{B})=\langle\mathrm{W}, \mathrm{R}, \mathrm{V}\rangle$ out of it. Now we show that $\operatorname{Card}(\mathrm{W}) \leq \operatorname{Card}(\mathrm{U})$ (we consider $\mathfrak{N}$ as already conservatively extended in progress of constructing $\mathcal{B})$. We set a function $f: U \longrightarrow \mathrm{W}$ as follows:

$$
f(\mathbf{w})= \begin{cases}{[i]_{\approx_{\mathcal{B}}},} & \text { if there is } i: i \in \mathcal{B} \text { such that } \\ & i \text { was affixed to } \mathbf{w} \text { during the } \\ & \text { derivation } \\ \text { arbitrary element of } \mathbf{W}, & \text { otherwise. }\end{cases}
$$


$f$ is injective and if we cut it to these elements of $U$ to which we assigned a nominal during the derivation, it is also an isomorphism. That concludes the proof.

To conclude our considerations it is sufficient to prove that the logic $\mathcal{H}_{\text {b-f }}$ has the finite model property. The following proposition deals with it.

Proposition 7.13. The logic $\mathcal{H}_{\mathrm{b}-\mathrm{f}}$ has the effective finite model property with the bounding function $\mu=2^{\operatorname{Card}(\operatorname{Sub}(\varphi))+1}$, where $\operatorname{Sub}(\varphi)$ is a set of all subformulas of a formula $\varphi$, i.e., whenever $\varphi$ has a model, it also has a model of the size not exceeding $2^{\operatorname{Card}(\operatorname{Sub}(\varphi))+1}$.

Proof. We use the standard, filtration-based argument. Suppose that a formula $\varphi$ is satisfied on a (possibly infinite) model $\mathfrak{M}=\langle W, R, V\rangle$. It means that there exists $w \in W$ such that $\mathfrak{M}, w \models \varphi$. We show that there exists a finite model $\mathfrak{M}^{\prime}$ that satisfies $\varphi$ and whose cardinality does not exceed $2^{\operatorname{Card}(\operatorname{Sub}(\varphi))+1}$.

First, we set the relation $\varkappa_{\varphi}$ on $W$ in the following way:

$w \leadsto \varphi v \quad$ iff: $\quad$ for all $\psi \in \operatorname{Sub}(\varphi) \mathfrak{M}, w \models \psi$ iff $\mathfrak{M}, v \models \psi$.

It is straightforward that $\varkappa_{\varphi}$ is an equivalence relation.

Now we are ready to construct our finite model that will satisfy $\varphi$. Let $\mathfrak{M}^{\prime}=\left\langle W^{\prime}, R^{\prime}, V^{\prime}\right\rangle$ such that:

$W^{\prime}=W / \min _{\varphi} \uplus W / \min _{\varphi}$

$R^{\prime}=\left\{\left\langle|v|_{\min _{\varphi}}|u|_{\operatorname{mi}_{\varphi}}\right\rangle \mid R(v, u)\right\}$,

$V^{\prime}(p)=\left\{|v|_{m_{\varphi}} \mid v \in V(p)\right\}$ for all proposition letters in $\varphi$,

$V^{\prime}(i)=\left\{|v|_{\operatorname{mon}_{\varphi}} \mid v \in V(i)\right\}$ for all nominals in $\varphi$.

We prove that $\mathfrak{M}^{\prime}$ satisfies $\varphi$ by induction on the complexity of subformulas of $\varphi$. Since the proof for the modal part of $\mathcal{H}_{\mathrm{b}-\mathrm{f}}$ is well known (cf. [15]) and the case of $\psi=i$ follows immediately from the definition of $V^{\prime}$, we confine ourselves to proving the cases of @ $@_{i}, \mathrm{E}_{X}, \mathrm{D}_{X}$ and $\diamond^{-} \chi$. In the forthcoming part of the proof we abbreviate $|\cdot| \operatorname{mon}_{\varphi}$ as $|\cdot|$. 
$\psi=@_{i} \chi$. Suppose that $\mathfrak{M}, v \Vdash @_{i} \chi$. It means that $\chi$ holds at a world $u$ at which also $i$ holds. This world is transformed to a singleton equivalence class $\{u\}$ in $W^{\prime}$. By the inductive hypothesis it follows that $\mathfrak{M}^{\prime},\{u\} \Vdash i$ and $\mathfrak{M}^{\prime},\{u\} \Vdash \chi$. Hence $\mathfrak{M}^{\prime},|v| \Vdash @_{i} \chi$.

$\psi=\mathrm{E}_{\chi}$. Suppose that $\mathfrak{M}, v \Vdash \mathrm{E} \chi$. It means that there exists a world $u$ at which $\chi$ holds. By the inductive hypothesis $\mathfrak{M}^{\prime},|u| \Vdash \chi$. Hence $\mathfrak{M}^{\prime},|v| \Vdash \mathrm{E}_{\chi}$.

$\psi=\mathrm{D}_{\chi}$. Suppose that $\mathfrak{M}, v \Vdash \mathrm{D}_{\chi}$. It means that there exists a world $u$ different than $v$, at which $\chi$ holds. By the inductive hypothesis $\mathfrak{M}^{\prime},|u| \Vdash \chi$. Two complementary cases might occur. If $|v| \neq|u|$, then we obtain $\mathfrak{M}^{\prime},|v| \Vdash \mathrm{D}_{\chi}$. If, however, $|v|=|u|$, it means that $\chi$ is also satisfied by a copy of $|v|$ that we pasted to $W^{\prime}$ at the stage of the construction of $\mathfrak{M}^{\prime}$. Since $|v|$ and its copy are distinct, we obtain $\mathfrak{M}^{\prime},|v| \Vdash \mathrm{D} \chi$.

$\psi=\diamond^{-} \chi$. Suppose that $\mathfrak{M}, v \Vdash \nabla^{-} \chi$. It means that $\chi$ holds at a world $u$ such that $u R v$. By the inductive hypothesis it follows that $\mathfrak{M}^{\prime},|u| \Vdash \chi$ and by the definition of $R^{\prime}$ we have that $|u| R^{\prime}|v|$. Hence $\mathfrak{M}^{\prime},|v| \Vdash \diamond^{-} \chi$.

Observe that pasting a distinct copy of $W / m_{\varphi}$ to $W^{\prime}$ is only necessary if $\mathrm{D}$ is involved. Therefore, in other cases the bounding function $\mu=2^{\operatorname{Card}(\operatorname{Sub}(\varphi))}$.

Of course, proving the above result for $\mathcal{H}_{\text {b-f }}$ defined over remaining considered classes of frames is only a matter of routine.

Consequently, we obtain the following result:

THEOREM 7.14. $\mathcal{T}_{\mathcal{H}_{b-f}}^{i}$ is terminating.

Proof. Follows from Corollary 7.11, Lemma 7.12 and Proposition 7.13.

Obviously, the bounding function $\mu$ from Proposition 7.13 can be reduced (cf. $[67,3]$ ), however, the logic $\mathcal{H}_{\text {b-f }}$ defined over the class of arbitrary frames (as well as reflexive, symmetric, transitive and serial) does not enjoy the polysize model property, therefore for some formulas 
the smallest possible model on which they are satisfied is still exponential in their size. It means that we cannot obtain any improvement in the complexity of the calculus. It will still be running in NEXPTIME. The case is different for the logic $\mathcal{H}_{\text {b-f }}$ defined over the classes of Euclidean and equivalential frames. As we can see in [68], for equivalential frames (and what is the most likely, for Euclidean frames too) $\mathcal{H}_{\text {b-f }}$ has the polysize model property. However, since the (ub) rule guarantees the great "pay-as-you-go" complexity ${ }^{6}$, in that case the calculus will be running in NP.

Lemmas 7.12 and 7.13 allow us turning our indeterministic decision procedure based on $\mathcal{T}_{\mathcal{H}_{\text {b-f }}}^{\mathrm{i}}$ into the deterministic one. We do so by abiding the following condition:

(det 1 ) Expand a branch of $\mathcal{T}_{\mathcal{H}_{\mathrm{b}-\mathrm{f}}}$-tableau until the number of equivalence classes of individuals in $\mathcal{B}$ exceeds the bound given by the $\mu$ function. Then stop.

\subsection{REMARKS ON BLOCKING MECHANISMS}

At the end of the present chapter, we would like to briefly answer the question of whether the unrestricted blocking mechanism is not using a cannon to kill a fly? Indeed, it is a powerful device that subsumes all blocking mechanisms exploited in tableau calculi for logics with the finite model property. However, some cost must be borne. As an explicit tableau rule, unrestricted blocking introduces an additional branching factor to the derivation. We consider two concise examples of tableau calculi for hybrid logics with blocking mechanisms different than ours.

\subsubsection{Subset blocking, equality blocking}

In [16] and [19] Blackburn, Bolander and Braüner provide a prefixed tableau calculus for the logic $\mathcal{H}\left(@, E, \diamond^{-}\right)$and an internalized calculus for $\mathcal{H}(@)$. They tailor the latter calculus in such a way that

6 It means that the complexity of a calculus supplied with the (ub) rule depends on the size of a bounding function from a statement of the finite model property for an underlying logic. If the bounding function is polynomial in the size of a formula, so is the size of one of tableau branches. Hence NP-completeness in that case. 
it does not produce infinite derivations. Therefore, the internalized tableau procedure will not be a subject of our considerations. Let's focus on the prefixed version. It is taken from Tzakova's paper [93]. We will not refer to the calculus as a whole since most of its rules are standard $^{7}$.

\subsubsection{The case of $\mathcal{H}$ and $\mathcal{H}$ (@)}

The thing that mostly distinguishes our calculus from that of Blackburn et $a l$. is that in the latter rules for @ and the $(\neg)$ rule explicitly introduce nominals to a branch (as well as new prefixes). It is significant enough to interfere with termination of a calculus. The rules in question look as follows:

$$
\text { (@) } \left.\frac{\sigma @_{i} \varphi}{\tau i, \tau \varphi} \quad \text { ( } @\right) ~ \frac{\sigma \neg @_{i} \varphi}{\tau i, \tau \neg \varphi}
$$

and

$$
(\neg) \frac{\sigma \neg i}{\tau i}
$$

where $\sigma$ and $\tau$ are meta-language labels for worlds and in both rules $\tau$ is fresh on a branch. Together with the rule handling the identity between worlds:

$$
\text { (Id) } \frac{\sigma \varphi, \sigma i, \tau i}{\tau \varphi}
$$

it can lead to infinite derivations. For consider a formula $i \wedge \diamond i$. The $\diamond$ operator creates a new label where $i$ is propagated. After applying (Id) we again obtain a $\diamond$ formula with $i$ under its scope. The derivation is performed all over again. Fresh labels are being indefinitely introduced to a branch despite the fact that they label the same world. It means that the derivation is infinite even though the model extracted from a branch is finite!

Blackburn and Bolander remove this flaw from the calculus by introducing a precedence order $\prec_{\mathcal{B}}$ over the set of all (meta-language) labels that occurred on a branch $\mathcal{B}$ during a derivation. The ordering relation is analogous to that introduced in Definition 7.4. $\sigma \prec_{\mathcal{B}} \tau$ whenever $\tau$

7 As we mentioned beforehand, in tableau calculi the shape of rules follows directly from the semantics of an underlying logic. Therefore, main differences in various tableau calculi lie in blocking mechanisms. 
was introduced to a branch by an applications of a generating rule to $\sigma \varphi$. We denote the reflexive, transitive closure of $\prec_{\mathcal{B}}$ by $\prec_{\mathcal{B}}^{*}$. Afterwards, they augment the $(\mathrm{Id})$ rule with the appropriate side-condition:

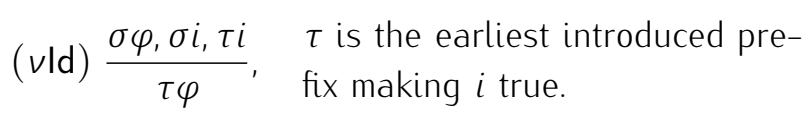

Clearly, (vld) only allows propagating formulas backwards, i.e. to the unique prefix which is the least element with respect to $\prec_{\mathcal{B}}$ at which the nominal from the current prefix holds. Still, retaining the possibility of transferring nominals between prefixes is essential for applications of the side condition of $(v \mathrm{ld})$. It is done by introducing a nominal variant of (Id):

$$
\text { (Nom) } \frac{\sigma i, \sigma j, \tau i}{\tau j}
$$

which is not restricted by any side-conditions. The calculus enriched in this fashion does not yield infinite derivations and preserves completeness. Before we state a formal completeness theorem for this calculus, we recall the following definition:

Definition 7.7 (Nominal urfather $([16])$ ). Let $\mathcal{B}$ be a branch of a tableau, and let $\sigma$ be a prefix occurring in $\mathcal{B}$. The nominal urfather of $\sigma$ in $\mathcal{B}$, written $s_{\mathcal{B}}(\sigma)$, is defined as follows

(1) If no nominals are true at $\sigma$ on $\mathcal{B}$, then $s_{\mathcal{B}}(\sigma)=\sigma$.

(2) Otherwise $s_{\mathcal{B}}(\sigma)$ is the earliest introduced prefix on $\mathcal{B}$ which makes some nominal true that $\sigma$ also makes true.

A prefix $\sigma$ is called a nominal urfather in $\mathcal{B}$ if $\sigma=s_{\mathcal{B}}(\tau)$ for some prefix $\tau$.

TheOREM 7.15 (Completeness ([16])). Let $\mathcal{B}$ be an open, fully-expanded branch in the calculus for $\mathcal{H}(@)$. For any formula $\sigma \varphi$ on $\mathcal{B}$ where $\sigma$ is a nominal urfather we have $\mathfrak{M}(\mathcal{B}), \sigma \Vdash \varphi$. In particular, we have $\mathfrak{M}(\mathcal{B}), \sigma_{0} \Vdash \varphi_{0}$, where $\sigma_{0}$ is the root of the branch.

Restoring termination for the initial version of the calculus was achieved by introducing a new rule and restricting the application range of one of the existing ones. Comparing to our prefixed calculus 
this seems to be a lot of effort since $\mathcal{T}_{\mathcal{H}_{\text {b-f }}}^{\mathrm{p}}$ restricted to the @ operators (i.e. without $E, D$ and $\diamond^{-}$) is terminating even when the unrestricted blocking rule is not involved! It is thanks to the fact that the whole equality reasoning is performed at the level of domain sort expressions where no nominals treated as formulas are involved.

\subsubsection{The case of $\mathcal{H}(@, E)$}

As displayed in Fig. 18, enriching a calculus with E can lead to generating infinite branches which yield infinite models (i.e. not only renaming the same world infinitely many times). It follows that devices introduced in the previous section are no longer sufficient to ensure termination of the calculus ${ }^{8}$. Therefore, some sort of additional blocking technique becomes indispensable. Blackburn et al. bring in the so-called loopchecking mechanism. Before we informally explain what it consists of, let's formulate the following definition:

Definition 7.8 (Inclusion urfather $([16])$ ). Let $\mathcal{B}$ be a branch of a tableau. We define the inclusion urfather of a prefix $\sigma$ in $\mathcal{B}$, written $u_{\mathcal{B}}(\tau)$, to be the earliest introduced prefix $\tau$ for which $L_{\mathcal{B}}(\sigma) \subseteq L_{\mathcal{B}}(\tau)$, where $L_{\mathcal{B}}(\sigma)$ is the set of all formulas that hold at a prefix $\sigma$ on a branch $\mathcal{B}$. A prefix $\sigma$ is called an inclusion urfather in $\mathcal{B}$ if $\sigma=u_{\mathcal{B}}(\tau)$ for some prefix $\tau$.

Now we fix the condition that restricts applications of prefix generating rules, namely $(\diamond)$ and $(E)$.

(R) A prefix generating rule is only allowed to be applied to a formula $\sigma \varphi$ on a branch if $\sigma$ is an inclusion urfather on that branch.

The intuition standing behind the condition above is that whenever a prefix $\sigma$ such that $L_{\mathcal{B}}(\sigma) \subseteq L_{\mathcal{B}}(\sigma)$ for some prefix $\tau$ is already present in $\mathcal{B}$, we can treat $\sigma$ and $\tau$ as prefixes labelling the same world. Following this trail, if some formula of the form $\nabla \varphi$ or $\mathrm{E} \varphi$ holds at $\sigma$, it also holds at $\tau$, thus already having been subjected to the application of $(\diamond)$ or $(E)$ respectively. In the model $\mathfrak{M}(\mathcal{B})$ resulting from a branch

8 Which is a straightforward result of the fact that infinite branches can be yielded in which no nominals occur (see Fig. 18). 
1.

2.

3.

4.

5.

6.

7.

8.

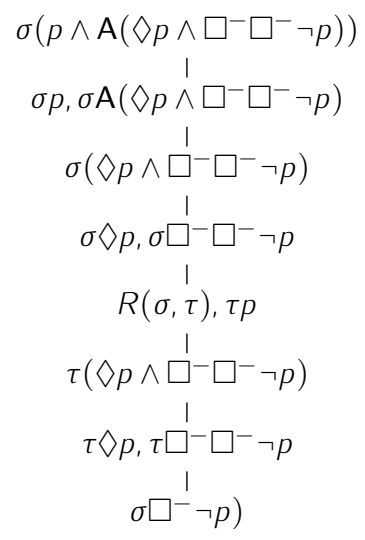

input

$(\wedge): 1$

$(\neg \mathrm{E}),(\neg \neg): 2$

$(\wedge): 3$

$(\diamond): 4$

$(\neg \mathrm{E}),(\neg \neg): 2,5$

$(\wedge): 6$

$\left(\neg \diamond^{-}\right),(\neg \neg): 7$

Figure 19: Formula $p \wedge \mathrm{A}\left(\diamond p \wedge \square^{-} \square^{-} \neg p\right)$ breaking the completeness of the calculus with with restriction $(\mathrm{R})$ for the logic $\mathcal{H}\left(@, \mathrm{E}, \diamond^{-}\right)$.

$\mathcal{B}$ we direct the accessibility arrow departing from the predecessor of $\sigma$ to $\tau$, creating a loop. Such a calculus is terminating and complete:

THEOREM 7.16 (Completeness ([16])). The calculus for $\mathcal{H}(@, E)$ with the restriction $(R)$ is complete.

Note that in our calculus even better effects can be obtained in terms of the size of a yielded model. Since we can freely compare between all prefixes that occurred within a branch, it can occur that we successfully merge two prefixes $\sigma$ and $\tau$ (in our case rather $x$ and $y$ ) such that $L_{\mathcal{B}}(\sigma) \cap L_{\mathcal{B}}(\tau)=\varnothing$, which is not possible in the Blackburn's et al. calculus.

\subsubsection{The case of $\mathcal{H}\left(@, E, \diamond^{-}\right)$}

However, that is not the end of our problems. Adding $\nabla^{-}$to our logic generates further trouble in assuring termination of the tableau procedure. In [16], the authors find a counterexample which shows that the notion of inclusion urfather and the restriction formulated upon it do not suffice as a blocking mechanism any more (see Fig. 19). It ensures termination of the calculus but instead it breaks the completeness. 
Admittedly, it does not yield infinite branches but completeness is lost. Therefore, a necessity occurs to modify our notion of urfather, which we do next.

Definition 7.9 (Quasi-urfather $([16])$ ). Let $\mathcal{B}$ be a branch of a tableau. If two prefixes $\sigma$ and $\tau$ make the same formulas true on $\mathcal{B}$, we call them twins in $\mathcal{B}$. A quasi-urfather in $\mathcal{B}$ is a prefix $\sigma$ for which there are no pair of distinct twins $\tau$, $\rho$ such that $\tau, \rho \prec_{\mathcal{B}} \sigma$.

Basing on the definition above, we restrict applications of generating rules as follows:

(D) A prefix generating rule is only allowed to be applied to a formula $\sigma \varphi$ on a branch if $\sigma$ is a quasi-urfather on that branch.

Thanks to (D) the termination of the calculus is retained - we only restricted the condition of merging prefixes. Now they must satisfy exactly the same set of formulas. For the proof of the completeness we need an additional notion:

Definition 7.10 (Identity urfather $([16])$ ). Let $\mathcal{B}$ be a branch of a tableau and let $\sigma$ be a prefix occurring in $\mathcal{B}$. The identity urfather of $\sigma$ in $\mathcal{B}$, written $v_{\mathcal{B}}(\sigma)$ is the earliest introduced prefixed $\tau$ satisfying:

1. $\tau$ is a twin of $\sigma$,

2. $\tau$ is a quasi-urfather.

If such a prefix does not exist, we leave $v_{\mathcal{B}}(\sigma)$ undefined. Thus $v_{\mathcal{B}}$ is only a partially defined mapping. A prefix $\sigma$ is called an identity urfather in $\mathcal{B}$ if $\sigma=v_{\mathcal{B}}(\tau)$ for some prefix $\tau$.

So equipped, we can state the following theorem:

TheOREM 7.17 (Completeness ([16])). Let $\mathcal{B}$ be an open, fully-expanded branch in the calculus for the logic $\mathcal{H}\left(@, E, \diamond^{-}\right)$with restriction $(D)$. For any formula $\sigma \varphi \in \mathcal{B}$ where $\sigma$ is an identity urfather we have $\mathfrak{M}(\mathcal{B}), \sigma \Vdash \varphi$. In particular, we have $\mathfrak{M}(\mathcal{B}), \sigma_{0} \Vdash \varphi_{0}$, where $\sigma_{0} \varphi_{0}$ is the root of the tableau.

Again, our calculus supplied with the unrestricted blocking rule handles inverse modality at ease. We try any possible merging anyway, so 
we do not need to introduce additional conditions once new operators occur in an underlying logic. As long as this logic enjoys the finite model property and a formula $\varphi$ itself is satisfiable, the (ub) rule will guide us to find a finite (minimal) model for $\varphi$.

Since the urfather of a particular prefix $\sigma$ is necessarily its ancestor with respect to $\prec_{\mathcal{B}}^{*}$, we call blocking mechanisms employed throughout this section ancestor blocking mechanisms.

\subsubsection{Pattern-based blocking}

In [56] Kaminski and Smolka present a terminating tableau calculus for the logic $\mathcal{H}\left(E, D, \diamond^{-}\right)$. They employ a brand new way of ensuring termination of their calculus, namely pattern-based blocking. This blocking mechanism is described in detail in Section 8.6.1 of Chapter 8 where we present a tableau calculus for the logic $\mathcal{M}\left(\mathrm{E}_{n}\right)$ also exploiting pattern-based blocking. We therefore confine ourselves to a short comment.

First, let's jump ahead a bit and define a pattern:

Definition 7.11 (Pattern). Given a branch $\mathcal{B}$, by a pattern $P$ we understand a set of expressions of the form $\square \varphi$ or $\nabla \varphi$ occurring in $\mathcal{B}$. Without loss of generality, we assume that each pattern only contains one $\diamond$ formula. By $P_{\varphi}^{i}$ we denote a set $\{\nabla \varphi\} \cup \square(i)$ such that $\nabla \varphi \in \diamond(i)$. We call a pattern $P$ expanded if there exist labels $i, j$ such that $P \subseteq P_{\varphi}^{i}$ for some $\varphi$, and $j: \varphi, R(i, j) \in \tilde{\mathcal{B}}$, where $\square(i)$ is the set of all $\square$ formulas that hold at a world labelled by $i, \nabla(i)$ is the set of all $\diamond$ formulas that hold at a world labelled by $i$ and $\tilde{\mathcal{B}}$ is the equational closure of a branch $\mathcal{B}$ (for the definition of equational closure see Section 8.6.1 of Chapter 8). We say that the label $i$ expands $P$ on $\mathcal{B}$.

A simple idea of Kaminski and Smolka assumes that before each application of a generating rule to a prefix $\sigma$, which introduces a new prefix $\tau$ to a branch, we check whether there exists any prefix $\rho$ that already expands a pattern of $\sigma$ and that can be linked to $\sigma$ as its successor. If our search fails, we can apply a rule to $\sigma$. If there exists a prefix containing an expansion set for a pattern of $\sigma$, we do not apply our rule to $\sigma$. Since we seek potential successors of $\sigma$ among all prefixes 
on a branch, not necessarily only those preceding $\sigma$ with respect to $\prec_{\mathcal{B}^{\prime}}^{*}$ we call pattern-based blocking an everywhere blocking mechanism. It works efficiently, especially if we augment it with some special searching strategies like the lazy branching strategy, however it faces several difficulties. First, we need to be cautious if we impose some restrictions on an accessibility relation (e.g. transitivity, reflexivity, symmetry etc.). As shown in Section 8.6.1, after switching the accessibility relation into a transitive or a reflexive one, we need to notably interfere with tableau rules and the notion of quasi-evidence which is a key notion in the termination proof for the calculi involving pattern-based blocking (both for $M E n$ and $\mathcal{H}\left(E, D, \diamond^{-}\right)$).

Second, if the inverse modality is involved in an underlying logic, pattern-based blocking cannot be an everywhere blocking strategy any more and needs to become an ancestor blocking strategy (which Kaminski and Smolka call chain-based blocking). Potential successors of a prefix $\sigma$ are only searched among $\prec_{\mathcal{B}}^{*}$-predecessors of $\sigma$.

This shows how careful we need to be when extending the logic we design a calculus for with additional operators or frame conditions. It might turn out that a blocking mechanism which has worked well so far, does not guarantee the termination of a calculus any longer or it causes a loss of completeness.

\subsection{CONCLUDING REMARKS}

In this chapter, we presented a prefixed and an internalised tableaubased decision procedure for the logic $\mathcal{H}_{\text {b-f. }}$ Both tableau calculi $\mathcal{T}_{\mathcal{H}_{\text {b-f }}}^{\mathrm{p}}$ and $\mathcal{T}_{\mathcal{H}_{\text {b-f }}}^{\mathrm{i}}$ were proven to be sound, complete and terminating.

Even though in the existing literature of the subject several approaches to systematic treatment of decision procedures for hybrid logics can be found, their main concern seems to be different from ours. The attempts of Blackburn, Bolander and Braüner are focused on tailoring a suitable tableau calculus for each hybrid logic separately. Therefore, they introduce two different ancestor blocking mechanisms, namely subset blocking and equality blocking for the logics, respectively, $\mathcal{H}$ (@) and $\left.\mathcal{H}\left(@, E, \diamond^{-}\right)\right)$and modify the notion of urfather subject to a par- 
ticular logic. The resulting calculus is conceptually complex but seems to avoid any superfluous performances of the rules.

Götzmann, Kaminski and Smolka in $[56,40]$ bring into being a calculus (and an automated prover) for quite a powerful hybrid logic $\mathcal{H}\left(\mathrm{E}, \mathrm{D}, \diamond^{-}\right)$. They also appropriately adapt their blocking mechanism, subject to the presence of particular operators or frame conditions.

The decision procedures introduced in this chapter present an approach which is different from the aforementioned. They introduce (ub) as an explicit tableau rule which is sound and, together with the conditions (c1)-(c4), ensures termination of the whole calculus. (ub) allows a direct comparison of every pair of labels that occurred on a branch and, therefore, subsumes any other blocking mechanisms (provided that the finite model property holds for a logic). (ub) is a generic rule which means that it generates every possible configuration of labels occurring on a branch. In comparison to [16] and [40], many of these configurations are superfluous. However, a huge advantage of this approach is conceptual simplicity which allows to avoid introducing complicated strategies of searching for a pair of labels that are liable to the blocking mechanism. We do not need to keep track of all changes brought to a calculus by augmenting a logic with additional operators or frame conditions as long as it still enjoys the finite model property - in all these cases (ub) suffices to ensure termination and retain completeness. Additionally, for each satisfiable formula $\varphi(\mathrm{ub})$ ensures that a minimal model for $\varphi$ (in terms of a domain size) will be generated, which cannot be guaranteed by the systems of [16] and [40]. Moreover, $\mathcal{T}_{\mathcal{H}_{\text {b-f }}}^{\mathrm{p}}$ and $\mathcal{T}_{\mathcal{H}_{\text {b-f }}}^{\mathrm{i}}$ provide a uniform approach to all hybrid logics mentioned in the chapter and covers the case of the difference modality which is omitted in [16]. 
DEDUCTIVE SYSTEMS FOR NON-STANDARD HYBRID LOGICS

In the chapter, we provide tableau-based decision procedures for the logic $\mathcal{M}\left(\mathrm{E}_{n}\right)$. In the existing literature, several approaches for deciding modal/description logics with counting operators can be found. [6] describes a decision procedure for modal logics with counting operators that exploits the translation function from the modal counting language to the hybrid language with the universal modality $\mathcal{H}(\mathrm{A})$. Tableau-based decision procedures for analogs of modal logics with counting operators were established in the field of description logics, where counting operators are known under the guise of cardinality constraints. Sound, complete and terminating tableau-calculi for these logics can be found in $[65,60,31]$. These calculi, in general, do not differ in the rules for cardinalities, however, they utilise different blocking mechanisms for ensuring termination, such as pairwise blocking or pattern-based blocking.

We exploit the framework from [84] to synthesise a sound, complete and terminating prefixed tableau calculus for $\mathcal{M}\left(E_{n}\right)$. We also provide a refinement of this calculus consisting in internalizing the semantics of the logic within the language of the logic. We only exploit global counting operators to dispense with meta-linguistic expressions like $\mathfrak{M}, x \models \varphi$ or $R(x, y)$ to achieve a full internalization.

We also describe a successful implementation of the calculus using the METTEL tableau prover generator $[90,1]$.

The chapter is structured as follows. In Section 8.1, we introduce a prefixed tableau calculus $\mathcal{T}_{\mathcal{M}\left(\mathrm{E}_{n}\right)}^{p}$ for the logic $M E n$. We also prove soundness and completeness of the calculus. Termination of $\mathcal{T}_{\mathcal{M}\left(\mathrm{E}_{n}\right)}^{p}$ is proven in Section 8.2. Section 8.3 is devoted to possible refinements of particular rules of $\mathcal{T}_{\mathcal{M}\left(\mathrm{E}_{n}\right)}^{p}$. The internalized version of the calculus named $\mathcal{T}_{\mathcal{M}\left(\mathrm{E}_{n}\right)}^{i}$ is presented in Section 8.4. We briefly comment on a successful implementation of $\mathcal{T}_{\mathcal{M}\left(E_{n}\right)}^{P}$ on METTER prover. Section 8.6 is 
an extensive overview of other decision procedures for logics with global counting operators. In Subsection 8.6.3, we provide, to the best of our knowledge, the first hybrid tableau algorithm involving the inequality solver that deals with global cardinality restrictions in an explicit form ${ }^{1}$.

\subsection{PREFIXED CALCULUS FOR $\mathcal{M}\left(\mathrm{E}_{n}\right)$}

In this section, we provide a sound and complete prefixed tableau calculus for the logic $\mathcal{M}\left(E_{n}\right)$. For a more extensive discussion on prefixed and internalized tableau calculi for hybrid logics, see Section 7.1 of Chapter 7.

\subsubsection{Meta-language for $\mathcal{M}\left(\mathrm{E}_{n}\right)$}

The meta-language of the tableau synthesis framework to specify the semantics of $\mathcal{M}\left(E_{n}\right)$ is a many-sorted first-order language. We denote it by $\mathrm{FO}\left(\mathcal{M}\left(E_{n}\right)\right)$. Since we have already given an extensive description of such a language for the logic $\mathcal{H}_{\mathrm{b}-\mathrm{f}}$ in Section 7.2.1 of Chapter 7, we restrict ourselves to two brief comments on features that distinguish $\mathrm{FO}\left(\mathcal{M}\left(\mathrm{E}_{n}\right)\right)$ from $\mathrm{FO}\left(\mathcal{H}_{\mathrm{b}-\mathrm{f}}\right)$. We also display the set $\boldsymbol{S}^{b}$ presenting the skolemized background theory for $\mathcal{M}\left(E_{n}\right)$ and the sets $S^{0}, S_{-}^{0}$ and $S_{+}^{0}$, which include first-order (respectively, positive skolemized first-order and negative skolemized first-order) formulas defining connectives of $\mathcal{M}\left(E_{n}\right)$ (see Figs. 20, 21 and 22).

Now for the remarks. First, the logic $\mathcal{M}\left(E_{n}\right)$, in contrast to $\mathcal{H}_{\text {b-f }}$, does not involve nominals. It means that there are no expressions of the sort 0 in the object language of $\mathcal{M}\left(E_{n}\right)$. It follows that we no longer need the predicate $v_{0}$ in our meta-language since there are no arguments it could be applied to. The only remaining $v$ predicate is $v_{1}$, which we henceforth denote simply as $v$.

Second, note that the rule for $E_{>n}$ in $S^{0}$ (Fig. 20) is in fact a scheme with $n$ as a parameter. It follows that it generates an infinite set $\Xi$ of actual $E_{>n}$ formulas, in principle causing an infinite blow-up of $S^{0}$.

1 The chapter is an extended and revised version of the joint manuscript by the author of the book with M. Khodadadi, R. Schmidt, and D. Tishkovsky ([62]). 


$$
\begin{aligned}
\mathrm{S}^{0}: & \forall x(v(\neg \varphi, x) \equiv \neg v(\varphi, x)) \\
\forall x(v(\varphi \wedge \psi, x) \equiv & (v(\varphi, x) \wedge v(\psi, x))) \\
\forall x(v(\diamond \varphi, x) \equiv & \exists y(R(x, y) \wedge v(\varphi, y))) \\
\forall x\left(v\left(\mathrm{E}_{>n} \varphi, x\right) \equiv\right. & \exists y_{1}, \ldots, y_{n+1}\left(\bigwedge_{0<i \leq n+1} v\left(\varphi, y_{i}\right)\right. \\
& \left.\left.\wedge \bigwedge_{0<i<j \leq n+1}\left(y_{i} \neq y_{j}\right)\right)\right)
\end{aligned}
$$

Figure 20: Semantic specification of connectives $S^{0}$ for the logic $\mathcal{M}\left(E_{n}\right)$.

$$
\begin{aligned}
& \mathrm{S}_{+}^{0}: v(\neg \varphi, x) \quad \rightarrow \quad \neg v(\varphi, x) \\
& v(\varphi \wedge \psi, x) \rightarrow(v(\varphi, x) \wedge v(\psi, x)) \\
& v(\diamond \varphi, x) \quad \rightarrow \quad(R(x, f(\diamond \varphi, x)) \wedge v(\varphi, f(\diamond \varphi, x))) \\
& v\left(\mathrm{E}_{>n} \varphi, x\right) \rightarrow\left(\bigwedge_{0<i \leq n+1} v\left(\varphi, f_{i}\left(\mathrm{E}_{>n} \varphi, x\right)\right)\right. \\
& \left.\wedge \bigwedge_{0<i<j \leq n+1}\left(f_{i}\left(\mathrm{E}_{>n} \varphi, x\right) \not f_{j}\left(\mathrm{E}_{>n} \varphi, x\right)\right)\right) \\
& \mathrm{S}_{-}^{0}: \neg v(\neg \varphi, x) \quad \rightarrow \quad v(\varphi, x) \\
& \neg v(\varphi \wedge \psi, x) \rightarrow(\neg v(\varphi, x) \vee \neg v(\psi, x)) \\
& \neg v(\diamond \varphi, x) \quad \rightarrow \quad(\neg R(x, y) \vee \neg v(\varphi, y)) \\
& \neg v\left(\mathrm{E}_{>n} \varphi, x\right) \rightarrow\left(\bigvee_{0<i \leq n+1} \neg v\left(\varphi, y_{i}\right) \vee \bigvee_{0<i<j \leq n+1}\left(y_{i} \approx y_{j}\right)\right)
\end{aligned}
$$

Figure 21: Sets $S_{+}^{0}$ and $S_{-}^{0}$ of Skolemised rules for connectives.

However, $\Xi$ is recursively enumerable so it can be turned into one tableau rule for $\mathrm{E}_{>n}$.

\subsubsection{The tableau calculus $\mathcal{T}_{\mathcal{M}\left(\mathrm{E}_{n}\right)}$}

The prefixed tableau calculus synthesised from the semantic specification provided in the previous section in Figs 21 and 22 is given in Fig. 23. We refer to it as $\mathcal{T}_{\mathcal{M}\left(\mathrm{E}_{n}\right)}^{p}$.

Since most of the rules have the same form as rules of the calculus $\mathcal{T}_{\mathcal{H}_{\text {b-f }}}^{\mathrm{p}}$, which were explained in detail in Section 7.2.2 of Chapter 7, we confine ourselves to providing an explanation for both the positive and the negative rule for $\mathrm{E}_{>n}$. 


$$
\begin{array}{rlr}
\mathrm{S}^{\mathrm{b}}: & x \approx x \quad x \approx y \rightarrow y \approx x & x \approx y \wedge y \approx z \rightarrow x \approx z \\
& (x \approx y \wedge v(\varphi, x)) \rightarrow v(\varphi, y) & x \approx y \rightarrow f(\varphi, x) \approx f(\varphi, y) \\
(x \approx y \wedge R(x, z)) \rightarrow R(y, z) & (x \approx y \wedge R(z, x)) \rightarrow R(z, y) \\
& & \\
(\text { ref }) x \approx x \rightarrow R(x, x) & (\text { sym }) R(x, y) \rightarrow R(y, x) \\
(\text { ser }) x \approx x \rightarrow R(x, f(x \approx x, x)) & (\operatorname{tran})(R(x, y) \wedge R(y, z)) \rightarrow R(x, z) \\
(\text { Eucl }) R(x, y) \wedge R(x, z) \rightarrow R(y, z) &
\end{array}
$$

Figure 22: Semantic specification of background theory $S^{b}$ for $\mathcal{M}\left(E_{n}\right)$.

The $\left(E_{>n}\right)$ rule reflects the semantics of the global counting operator. It is generic, since applied to $\mathrm{E}_{>n} \varphi$, it produces $n+1$ new, distinct worlds in which $\varphi$ holds. The intuitive meaning of $\left(\neg \mathrm{E}_{>n}\right)$ is as follows. Since it is not the case that there are more than $n$ worlds in which $\varphi$ holds, there are at most $n$ worlds in which $\varphi$ holds. So, for each $n+1$-tuple of worlds that have already appeared in the branch either some of them do not satisfy $\varphi$ or some of them are equal. Again, we use $y \approx y$ formulas to ensure the rule is applied only to domain terms occurring in the current branch. It follows that $\left(\neg \mathrm{E}_{>n}\right)$ is not applicable until at least $n+1$ worlds occur in the branch. We show in Section 8.3, the rule $\left(\neg \mathbf{E}_{>n}\right)$, contrary to the rule $(\neg \diamond)$, is not refinable by turning some of its conclusions to premises.

\subsubsection{Soundness and completeness of $\mathcal{T}_{\mathcal{M}\left(\mathrm{E}_{n}\right)}^{p}$}

In this section, soundness and completeness of $\mathcal{T}_{\mathcal{M}\left(\mathrm{E}_{n}\right)}^{p}$ is established. All necessary definitions, including the definition of soundness, completeness and constructive completeness for a calculus can be found in Section 7.2.3 of Chapter 7.

Suppose that $\varphi$ a $\mathcal{M}\left(E_{n}\right)$ formula. Let $\varphi$ be an input for our tableau calculus $\mathcal{T}_{\mathcal{M}\left(\mathrm{E}_{n}\right)}^{p}$. We denote a tableau for $\varphi$ by $\mathcal{T}_{\mathcal{M}\left(\mathrm{E}_{n}\right)}^{p}(\varphi)$. Let $\mathcal{B}$ be an open, fully-expanded branch of $\mathcal{T}_{\mathcal{M}\left(\mathrm{E}_{n}\right)}^{p}(\varphi)$. We associate with $\mathcal{B}$ a model $\mathfrak{M}(\mathcal{B})=\langle\mathrm{W}, \mathrm{R}, \mathrm{V}\rangle$ defined as follows:

$\mathrm{W}=\{\pi(x) \mid x$ is a term of the domain sort and $x \approx x \in \mathcal{B}\}$, 
Rules for connectives:

$$
\begin{aligned}
& (\neg) \frac{v(\neg \varphi, x)}{\neg v(\varphi, x)} \quad(\neg \neg) \frac{\neg v(\neg \varphi, x)}{v(\varphi, x)} \\
& (\wedge) \frac{v(\varphi \wedge \psi, x)}{v(\varphi, x), v(\psi, x)} \quad(\neg \wedge) \frac{\neg v(\varphi \wedge \psi, x)}{\neg v(\varphi, x) \mid \neg v(\psi, x)}
\end{aligned}
$$

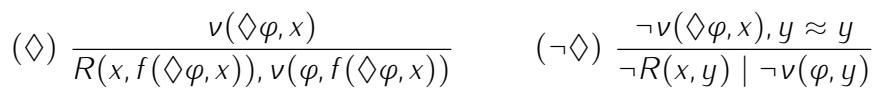

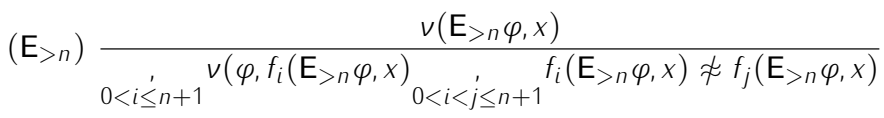

$$
\begin{aligned}
& \left(\neg \mathrm{E}_{>n}\right) \frac{\neg v\left(\mathrm{E}_{>n} \varphi, x\right)_{0<i \leq n+1} y_{i} \approx y_{i}}{\left.\right|_{0<i \leq n+1} \neg v\left(\varphi, y_{i}\right)_{0<i<j \leq n+1}\left(y_{i} \approx y_{j}\right)}
\end{aligned}
$$

Rules for equality:

$$
\begin{gathered}
\frac{v(\varphi, x)}{x \approx x} \quad \frac{x \approx y}{y \approx x} \quad \frac{x \approx y, y \approx z,}{y \approx z} \quad \frac{x \approx y, R(x, z)}{R(y, z)} \quad \frac{x \approx y, R(z, x)}{R(z, y)} \\
\frac{x \approx y, v(\varphi, x)}{v(\varphi, y)} \quad \frac{x \approx y}{f(\varphi, x) \approx f(\varphi, y)}
\end{gathered}
$$

Closure rules and unrestricted blocking rule:

$$
\frac{v_{1}(\varphi, x), \neg v_{1}(\varphi, x)}{\perp} \quad \frac{R(x, y), \neg R(x, y)}{\perp} \quad \frac{x \approx y, x \neq y}{\perp} \quad \text { (ub) } \frac{x \approx x, y \approx y}{x \approx y \mid x \neq y}
$$

Rules for frame conditions:

$$
\begin{aligned}
\text { (ref) } \frac{v(x \approx x)}{R(x, x)} \quad(\operatorname{sym}) \frac{R(x, y)}{R(y, x)} \quad(\operatorname{tran}) \frac{R(x, y), R(y, z)}{R(x, z)} \\
\text { (Eucl) } \frac{R(x, y), R(x, z)}{R(y, z)} \quad(\text { ser }) \frac{x \approx x}{R(x, f(x, x \approx x))}
\end{aligned}
$$

Figure 23: The rules of $\mathcal{T}_{\mathcal{M}\left(\mathrm{E}_{n}\right)}^{p}$ 
$\mathrm{R}=\{\langle\pi(x), \pi(y)\rangle \mid x, y$ are terms of the domain sort and $R(x, y) \in$ $\mathcal{B}\}$,

$\checkmark$ maps every propositional variable $p$ of the sort 1 to the set $\{\pi(x) \mid$ $v(p, x) \in \mathcal{B}\}$,

where $\pi$ is the canonical projection valuation defined as follows: $\pi(x)=[x]_{\sim \mathcal{B}}$ for every term $x$ of the domain sort that appeared in $\mathcal{B}$.

In the following lemma we prove that if there is an open and fullyexpanded branch $\mathcal{B}$ in $\mathcal{T}_{\mathcal{M}\left(\mathrm{E}_{n}\right)}^{p}(\varphi)$, then a model constructed in the way described above reflects $\mathcal{B}$. For all cases, save $\mathrm{E}_{>n} \psi$ and $\neg \mathrm{E}_{>n} \psi$, we refer the reader to the proof of Lemma 7.2 in Chapter 7, which was performed for the logic $\mathcal{H}_{\mathrm{b}-\mathrm{f}}$ and can be extended on the logic $\mathcal{M}\left(\mathrm{E}_{n}\right)$.

Lemma 8.1. Let $\varphi$ be an input set of $\mathcal{M}\left(E_{n}\right)$-formulas. Let $\mathcal{B}$ be an open, fully expanded branch of a tableau for $\Gamma$. Then $\mathfrak{M}(\mathcal{B})$ reflects $\mathcal{B}$, that is:

(i) for any $\mathcal{M}\left(\mathrm{E}_{n}\right)$-formula $\varphi$, if $v(\varphi, x) \in \mathcal{B}$ then $\mathfrak{M}(\mathcal{B}), \pi(x) \Vdash \varphi$,

(ii) if $R(x, y) \in \mathcal{B}$ then $\langle\pi(x), \pi(y)\rangle \in \mathbf{R}$,

(iii) if $x \approx y$ then $\pi(x)=\pi(y)$.

Proof. We proceed by induction on the complexity of $\varphi$.

$\varphi=E_{>n} \psi$. Because $\mathcal{B}$ is open and fully expanded, so $\left(E_{>n}\right)$-rule has been applied to $E_{>n} \psi$. Therefore, it follows that $v\left(\psi, x_{1}\right) \in$ $\mathcal{B}, \ldots, v\left(\psi, x_{n+1}\right) \in \mathcal{B}$ and $x_{i} \neq x_{j} \in \mathcal{B}$ for $0<i<j \leq n+1$. By induction hypothesis and by construction of $W$ we obtain that $\mathfrak{M}(\mathcal{B}), \pi\left(x_{i}\right) \mid=\psi$ for $i=1, \ldots, n+1$. Since $\mathcal{B}$ is open, $x_{i} \approx x_{j} \notin \mathcal{B}$ for $0<i<j \leq n+1$ and, hence, $\pi\left(x_{i}\right) \neq \pi\left(x_{j}\right)$ for $0<i<j \leq n+1$. By the definition of the $\Vdash$ relation this means that $\mathfrak{M}(\mathcal{B}), \pi(x) \Vdash \mathrm{E}_{>n} \psi$.

$\varphi=\neg \mathrm{E}_{>n} \psi$. Let $\pi\left(x_{1}\right), \ldots, \pi\left(x_{n+1}\right) \in \mathrm{W}$ and such that $\pi\left(x_{i}\right) \neq \pi\left(x_{j}\right)$ for $0<i<j \leq n+1$. It follows that $x_{i} \approx x_{i} \in \mathcal{B}$ for $i=1, \ldots, n+1$ and $x_{i} \neq x_{j} \in \mathcal{B}$ for $0<i<j \leq n+1$. Since the rules $(\neg)$ and $\left(\neg \mathrm{E}_{>n}\right)$ are applied to $v\left(\neg \mathrm{E}_{>n} \chi_{,} x\right)$ on $\mathcal{B}$ 
we have $\neg v\left(\psi, x_{i}\right) \in \mathcal{B}$ for some $i=1, \ldots, n+1$. By the inductive hypothesis we get $\mathfrak{M}(\mathcal{B}), \pi\left(x_{i}\right) \Vdash \neg \chi$. Since $n+1$-tuple of elements of $\mathrm{W}$ was picked arbitrarily, by the definition of $\mathfrak{M}(\mathcal{B})$ we obtain $\mathfrak{M}(\mathcal{B}), \pi(x) \Vdash \mathrm{E}_{>n} \psi$, whence $\mathfrak{M}(\mathcal{B}), \pi(x) \Vdash \neg \mathrm{E}_{>n} \psi . \quad-$

THEOREM 8.2. $\mathcal{T}_{\mathcal{M}\left(\mathrm{E}_{n}\right)}^{P}$ is a constructively complete tableau calculus for $\mathcal{M}\left(\mathrm{E}_{n}\right)$.

Proof. Follows from the construction of $\mathfrak{M}(\mathcal{B})$ and Lemma 8.1.

\subsection{TERMINATION OF THE CALCULUS}

Due to the presence of global operators, the calculus $\mathcal{T}_{\mathcal{M}\left(\mathrm{E}_{n}\right)}$ can generate infinite derivations for a satisfiable input set of formulas. A good example of such input is the formula $E_{=0}\left(E_{=0} \varphi\right)$ (see Fig. 24). No such problem occurs if the input set is unsatisfiable. The logic $\mathcal{M}\left(E_{n}\right)$ is embeddable into the two-variable fragment of first order logic with counting quantifiers and is therefore compact. It means that if an input is unsatisfiable any $\mathcal{T}_{\mathcal{M}\left(\mathrm{E}_{n}\right)}$-derivation eventually closes all branches. However, the presence of $\mathrm{E}_{>n}$-operators, which are universal modalities with numerical restrictions, can interfere with termination if a particular input set of formulas is satisfiable. A rule that causes difficulties is the $\left(\neg \mathrm{E}_{>n}\right)$ rule due to the fact that it spreads a particular content to all worlds that occurred in a branch up to the point of its application.

Various blocking techniques were already applied to logics with counting operators, mainly in the area of description logics. In [60], pattern-based blocking was used in logic $\mathcal{S H O Q}$ which can be considered as a more-expressive analog of the logic $\mathcal{M}\left(\mathrm{E}_{n}\right)$. In [65], pair-

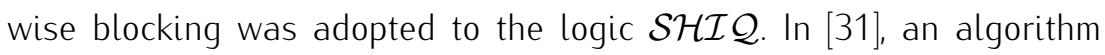
for $\mathcal{A L C Q}$ was presented in which no particular blocking mechanism was distinguished. Termination was ensured by an algorithm with hybrid character, exploiting an inequality solver to cope with numerical restrictions. We refer to these results more thoroughly in Section 8.6.

The blocking mechanism applied to the logic $\mathcal{M}\left(E_{n}\right)$ in this chapter, namely unrestricted blocking, is different than instruments introduced in the abovementioned papers. 


$\begin{array}{lrr}\text { 1. } & v\left(\mathrm{E}_{=0}\left(\mathrm{E}_{=0} \varphi\right), x\right) & \text { input } \\ \text { 2. } & v\left(\neg \mathrm{E}_{>0}\left(\neg \mathrm{E}_{>0} \varphi\right), x\right) & \text { rewriting: } 1 \\ \text { 3. } & x \approx x & (\approx): 2 \\ \text { 4. } & \neg v\left(\mathrm{E}_{>0}\left(\neg \mathrm{E}_{>0} \varphi\right), x\right) & (\neg): 2 \\ \text { 5. } & \neg v\left(\neg \mathrm{E}_{>0} \varphi, x\right) & \left(\neg \mathrm{E}_{>n}\right): 3,4 \\ \text { 6. } & v\left(\mathrm{E}_{>0} \varphi, x\right) & (\neg \neg): 4 \\ \text { 7. } & v\left(\varphi, f\left(\mathrm{E}_{>0} \varphi, x\right)\right) & \left(\mathrm{E}_{>n}\right): 5 \\ \text { 8. } & f\left(\mathrm{E}_{>0} \varphi, x\right) \approx f\left(\mathrm{E}_{>0} \varphi, x\right) & (\approx): 6 \\ \text { 9. } & v\left(\neg \neg \mathrm{E}_{>0} \varphi, f\left(\mathrm{E}_{>0} \varphi, x\right)\right) & \left(\neg \mathrm{E}_{>n}\right): 4,8 \\ & \quad \cdots & \\ & (\text { infinite derivation) } & \end{array}$

Figure 24: Formula $\mathrm{E}_{=0}\left(\mathrm{E}_{=0} \varphi\right)$ generating infinite $\mathcal{T}_{\mathrm{K}(\mathrm{E})_{n}}$-derivartion.

We now gather some information that already occurred in Chapter 7 and re-use it in establishing termination for the calculus $\mathcal{T}_{\mathcal{M}\left(\mathrm{E}_{n}\right)}^{p}$.

DEFINITION 8.1. Let $\leq_{\mathcal{B}}$ be an order of occurrences of expressions in a branch $\mathcal{B}$. The unrestricted blocking mechanism consists of the following rule and two strategy conditions added to the calculus:

$$
\text { (ub) } \frac{x \approx x, y \approx y}{x \approx y \mid x \neq y}
$$

(d1) The (ub)-rule should be applied exhaustively on a branch $\mathcal{B}$ before each application of the $\left(E_{>n}\right)$ and $(\diamond)$-rules,

(d2) If $\mathrm{E}_{>n^{-}}$or $\diamond$-formula $\varphi$ occurs in $x_{1}, \ldots, x_{n} \in[x]_{\approx}$, then the $\left(\mathrm{E}_{>n}\right)$ rule and $(\diamond)$ rule, respectively, are only applied to the least element in $[x] \approx$ with respect to $\leq_{\mathcal{B}}$.

Here, we assume that an input set of formulas $\Gamma$ is finite. As a consequence of conditions (d1) and (d2), we obtain an easy observation that for each $x$ occurring on $\mathcal{B}$ the $\left(E_{>n}\right)$ and $(\diamond)$-rules can be applied to elements of $[x] \approx$ only finitely many times, subject to the number of 
distinct $\mathrm{E}_{>n}$ and $\diamond$-formulas in $\Gamma$. It follows that a fully expanded, open $\mathcal{T}_{\mathcal{M}\left(\mathrm{E}_{n}\right)}^{\text {(ub) }}$-tableau branch $\mathcal{B}$ is finite iff $\mathfrak{M}(\mathcal{B})$ is finite.

Now we formulate a stronger result (cf. [83, Lemma 13]) that links arbitrary models and $\mathcal{T}_{\mathcal{M}\left(\mathrm{E}_{n}\right)}^{p}$-tableau for a satisfiable set of input formulas $\Gamma$. Namely, the following lemma states that if an input formula $\varphi$ has a finite model then any $\mathcal{T}_{\mathcal{M}\left(\mathrm{E}_{n}\right)}^{P}$-tableau derivation will include a branch in which the number of equivalence classes of worlds will not exceed the number of worlds in this model. This is a strict analog of Lemma 7.12 from Chapter 7.

Proposition 8.3. Let $\varphi$ be an arbitrary $\mathcal{M}\left(E_{n}\right)$-formula. Suppose that $\mathfrak{N}=\langle\mathrm{U}, \mathrm{S}, \mathrm{Z}\rangle$ is a model for $\varphi$. Then, there exists a branch $\mathcal{B}$ in $\mathcal{T}_{\mathcal{M}\left(\mathrm{E}_{n}\right)}^{P}$ tableau for $\varphi$ such that $\operatorname{Card}(\mathrm{U}) \geq \operatorname{Card}(\mathcal{B})$, where $\operatorname{Card}(\mathcal{B})=\operatorname{Card}(\{[x] \approx \mid x \approx x \in \mathcal{B}\})$.

Proof. We search for a branch $\mathcal{B}$ and $\mathfrak{M}(\mathcal{B})=\langle\mathrm{W}, \mathrm{R}, \mathrm{V}\rangle$ such that $\operatorname{Card}(\mathrm{W}) \leq \mathrm{Card}(\mathrm{U})$. We construct $\mathcal{B}$ inductively by applying the rules in derivation.

BASE CASE: We have $v(\varphi, x)$ in an initial step of our derivation. Since $\varphi$ is satisfied by $\mathfrak{N}$, there exists $\mathbf{x} \in U$ such that $\mathfrak{N}, \mathbf{x} \Vdash \varphi$. We set $x=\mathrm{x}$.

INDUCTIVE STEP: We assume that up to the $n$-th step of derivation we matched each expression of the domain sort that have occurred in the tableau with an appropriate world in $\mathfrak{N}$.

$(\wedge)$ Assume that in the $n$-th step of derivation we obtained a formula of the form $v(\psi \wedge \chi, x)$. By the inductive hypothesis, there exists $\mathrm{x} \in \mathrm{U}$ such that $\mathfrak{N}, \mathbf{x} \Vdash \psi \wedge \chi$. By the definition of a model, it follows that $\mathfrak{N}, \mathbf{x} \Vdash \psi$ and $\mathfrak{N}, \mathbf{x} \Vdash \chi$, so after applying $(\wedge)$ and obtaining $v(\psi, x)$ and $v(x, x)$, matching $x=x$ still holds.

$(\neg \wedge)$ We have a formula of the form $\neg v(\psi \wedge \chi, x)$. By the inductive hypothesis, there exists $\mathrm{x} \in \mathrm{U}$ such that $\mathfrak{N}, \mathrm{x} \Vdash \neg(\psi \wedge \chi)$. By the definition of a model, it follows that $\mathfrak{N}, \mathbf{x} \| \psi$ or $\mathfrak{N}, \mathbf{x} \neq \chi$. After applying $(\neg \wedge)$ which is a branching rule, we obtain $\neg \nu(\psi, x)$ on the left branch and $\neg v(X, x)$ on the second branch. We choose the left 
branch in the first case and the right branch in the second and leave the matching $\mathrm{x}=x$.

$(\diamond)$ In the $n$-th step of derivation we have a formula of the form $v(\diamond \psi, x)$. By the inductive hypothesis, there exists $\mathrm{x} \in \mathrm{U}$ such that $\mathfrak{N}, \mathbf{x} \Vdash \nabla \psi$ and, therefore, that there is $\mathrm{y} \in \mathrm{U}$ such that $\mathrm{x} \mathrm{S} y$ holds and $\mathfrak{N}, \mathbf{y} \Vdash \psi$. After applying $(\diamond)$ we obtain $R(x, f(\diamond \psi, x))$ and $v(\psi, f(\diamond \psi, x))$. We set $\mathbf{y}=f(\diamond \psi, x)$.

$(\neg \diamond)$ We have formulas of the form $\neg v(\diamond \psi, x)$ and $y \approx y$. Since $y$ must have occurred in $\mathcal{B}$ so far, we have already fixed a particular $\mathbf{y} \in \mathbf{U}$ that $\mathbf{y}=y$. By the inductive hypothesis, there exists $\mathbf{x} \in \mathbf{U}$ such that $\mathfrak{N}, \mathbf{x} \Vdash \neg \diamond \psi$ and, therefore, that either $\mathrm{xSy}$ does not hold or $\mathfrak{N}, \mathbf{x} \| \psi$. After applying $(\neg \diamond)$ being a branching rule we obtain $\neg R(x, y)$ and $\neg v(\psi, y)$. If the former is the case we choose the left branch, if the latter is the case we choose the right branch and leave the matching $\mathrm{x}=\mathrm{x}, \mathrm{y}=\mathrm{y}$.

$\left(E_{>n}\right)$ In the $n$-th step of derivation we have a formula of the form $v\left(\mathrm{E}_{>n} \psi, x\right)$. By the inductive hypothesis, there exists $\mathrm{x} \in \mathrm{U}$ such that $\mathfrak{N}, \mathrm{x} \Vdash \mathrm{E}_{>n} \psi$ and, therefore, that there is $n+1$-tuple of distinct worlds $\mathrm{x}_{1}, \ldots, \mathrm{x}_{\mathrm{n}+1} \in \mathrm{U}$ such that $\mathfrak{N}, \mathrm{x}_{1} \Vdash \psi, \ldots, \mathfrak{N}, \mathbf{x}_{\mathbf{n}+1} \Vdash \psi$. After applying $\left(\mathrm{E}_{>n}\right)$ we obtain and $v\left(\psi, f_{1}\left(\mathrm{E}_{>n} \psi, x\right)\right), \ldots$, $v\left(\psi, f_{n+1}\left(\mathrm{E}_{>n} \psi, x\right)\right)$ and $x_{i} \neq x_{j}$ for $0<i<j \leq n+1$. We set $x_{1}=f_{1}\left(E_{>n} \psi, x\right), \ldots, x_{n+1}=f_{n+1}\left(E_{>n} \psi, x\right)$.

$\left(\neg \mathrm{E}_{>n}\right)$ We have the following formulas $\neg v\left(\mathrm{E}_{>n} \psi, x\right)$, $y_{1} \approx y_{1}, \ldots, y_{n+1} \approx y_{n+1} . y_{1}, \ldots, y_{n+1}$ must have already occurred on $\mathcal{B}$, so we matched them with particular $y_{1}, \ldots, y_{n+1}$. By the inductive hypothesis, there exists $\mathrm{x} \in \mathrm{U}$ such that $\mathfrak{N}, \mathrm{x} \mathbb{H} \mathrm{E}_{>n} \varphi$. It means that either $\mathfrak{N}, \boldsymbol{y}_{\mathrm{i}} \| \boldsymbol{\psi} \varphi$ for some $i \in\{1, \ldots, n+1\}$ or $\mathrm{y}_{\mathrm{i}} \neq \mathrm{y}_{\mathrm{j}}$ for some $0<i<j \leq n+1$. After application of $\left(\neg \mathrm{E}_{>n}\right)$ we pick the correct branch, subject to the case that turned out to hold for $\mathfrak{N}$.

(ub) Two formulas $x \approx x$ and $y \approx y$ have already occurred on the branch. It means that we affixed two worlds $x, y \in U$ to them, respectively. It either is the case that $\mathbf{x}=\mathbf{y}$ or $\mathbf{x} \neq \mathbf{y}$. After applying 
(ub) we chose left branch if the former is the case and we chose the right branch if the latter holds.

Thus, we showed that if there is a $\Gamma$-model $\mathfrak{N}=\langle\mathbf{U}, \mathbf{S}, \mathbf{Z}\rangle$ then in a tableau derivation we can find a branch $\mathcal{B}$ and a model $\mathfrak{M}(\mathcal{B})=$ $\langle\mathrm{W}, \mathrm{R}, \mathrm{V}\rangle$, and subsequently fix a function $f: \mathrm{U} \longrightarrow \mathrm{W}$ defined as follows:

$$
f(\mathbf{x})= \begin{cases}{[x]_{\approx \prime}} & \text { if there is } x \approx x \in \mathcal{B} \text { such that } \\ & x \text { was affixed to } x \text { during the } \\ & \text { derivation } \\ \text { arbitrary element of } \mathbf{W}, & \text { otherwise. }\end{cases}
$$

By construction of $\mathfrak{M}(\mathcal{B})$ it follows that $f$ is onto. Hence, the conclusion.

Definition 8.2. We say that a tableau calculus $\mathcal{T}$ is terminating (for satisfiability) iff for every formula $\varphi$ every closed tableau $\mathcal{T}(\varphi)$ is finite and every open tableau $\mathcal{T}(\varphi)$ has a finite open branch.

To ensure termination for $\mathcal{T}_{\mathcal{M}\left(\mathrm{E}_{n}\right)}^{P}$ it suffices therefore to establish the finite model property for $\mathcal{M}\left(E_{n}\right)$, which we did in Theorem 5.16 in Chapter 5. It guarantees that for every satisfiable formula $\varphi$ there is a finite model satisfying it, which will be reflected by one of the branches of $\mathcal{T}_{\mathcal{M}\left(\mathrm{E}_{n}\right)}^{P}$. Since a model reflected by a branch is finite, a branch itself is finite too.

Hence, as a corollary to Proposition 8.3, Theorem 5.16 and the fact that each rule of $\mathcal{T}_{\mathcal{M}\left(\mathrm{E}_{n}\right)}^{p}$ is finitely branching we get:

THEOREM 8.4. $\mathcal{T}_{\mathcal{M}\left(\mathrm{E}_{n}\right)}^{(u b)}$ is terminating.

To provide a deterministic decision procedure involving $\mathcal{T}_{\mathcal{M}\left(\mathrm{E}_{n}\right)}^{p}$ we formulate the following condition for the derivation strategy:

(det2) Expand a branch of a $\mathcal{T}_{\mathcal{M}\left(\mathrm{E}_{n}\right)}^{p}$-tableau until the number of equivalence classes of worlds in $\mathcal{B}$ exceeds the bound from Theorem 5.16. Then stop. 
Since the logic $\mathcal{M}\left(E_{n}\right)$ is NEXPTIME-complete for the classes of arbitrary, reflexive, symmetric, serial and transitive frames, which was proven in Section 5.5 of Chapter 5, the calculus $\mathcal{T}_{\mathcal{M}\left(\mathrm{E}_{n}\right)}^{p}$ is complexity optimal for these classes of frames, as exploiting the exponential-size model property. The situation might be worse in the case of Euclidean and equivalential frames. The complexity of the logic $\mathcal{M}\left(E_{n}\right)$ over these classes is conjectured to be lower than NEXPTIME-complete (the most probably it is NP-complete). However, the logic $\mathcal{M}\left(E_{n}\right)$ only enjoys the exponential-size model property and lacks the polysize model property, which means that the performance of the derivation in $\mathcal{T}_{\mathcal{H}_{\text {b-f }}}^{\mathrm{p}}$ will not be complexity-optimal for these classes of frames if the conjecture happens to be true. However, it is not only a problem of the calculus $\mathcal{T}_{\mathcal{M}\left(\mathrm{E}_{n}\right)}^{p}$. In fact, every calculus which returns a description of a model when a satisfiable formula is given as an input, will perform not optimally (since for some formulas it will return a description of a model whose size will be exponentially larger than the size of a formula).

\subsection{RULE REFINEMENT OF $\mathcal{T}_{\mathcal{M}\left(\mathrm{E}_{n}\right)}$}

Since in the calculus $\mathcal{T}_{\mathcal{M}\left(\mathrm{E}_{n}\right)}^{p}$ a refinement of the $(\neg \diamond)$ rule is also possible, we refer the reader to Section 7.3 of Chapter 7 where we described in details the process of refining $(\neg \diamond)$. In this section, we limit ourselves to the comment on the (im)possibility of refining the rule $\left(\neg \mathrm{E}_{>n}\right)$.

Due to the large branching factor of the $\left(\neg E_{>n}\right)$ rule, its unrefinability to

$$
\frac{\neg v\left(\mathrm{E}_{>n} \varphi, x\right), v\left(\varphi, y_{1}\right), \ldots, v\left(\varphi, y_{n+1}\right)}{\prod_{0<i<j \leq n+1}\left(y_{i} \approx y_{j}\right)}
$$

is a significant loss. As an example, consider the input set $\Gamma=\left\{v\left(\neg \mathrm{E}_{>1} \neg p, x\right), v(q, y)\right\}$. No rules are applicable to $\Gamma$ therefore the derivation stops at the initial node. A model reflecting the branch $\mathfrak{M}(\mathcal{B})=\langle\mathrm{W}, \mathrm{R}, \mathrm{V}\rangle$ is defined by: $\mathrm{W}=\{\{x\},\{y\}\}, \mathrm{R}=\varnothing$, $\mathrm{V}(p)=\varnothing, \mathrm{V}(q)=\{y\}$. It therefore follows that both worlds $x$ and $y$ satisfy $\neg p$, so $\neg \mathrm{E}_{>1} \neg p$ is not satisfied by $\mathfrak{M}(\mathcal{B})$ although the formula is evidently satisfiable. 


$$
\begin{array}{llll}
\operatorname{Tr}_{\mathcal{H}}(v(\varphi, x)) & =@_{i_{x}} \varphi & \operatorname{Tr}_{\mathcal{H}}(\neg v(\varphi, x)) & =@_{i_{x}} \neg \varphi \\
\operatorname{Tr}_{\mathcal{H}}(R(x, y)) & =@_{i_{x}} \diamond j_{y} & \operatorname{Tr}_{\mathcal{H}}(\neg R(x, y)) & =@_{i_{x}} \neg \nabla j_{y} \\
\operatorname{Tr}_{\mathcal{H}}(x \approx y) & =@_{i_{x}} j_{y} & \operatorname{Tr}_{\mathcal{H}}(x \neq y y) & =@_{i_{x}} \neg j_{y}
\end{array}
$$

Figure 25: Hybrid translation function for domain-sort expressions.

\subsection{INTERNALIZED CALCULUS FOR $\mathcal{M}\left(\mathrm{E}_{n}\right)$}

A second refinement described in [84] allows the synthesised rules to be reformulated without the use of the $v$-predicate or any other domain sort symbols. This is possible for logics allowing the encoding of domain expressions by its own means. As described in Section 7.4 of Chapter 7, this can be done for hybrid logic with the @-operator. There are three basic cases of domain expressions (modulo negation): $v(\varphi, x), R(x, y), x \approx y$. Each of them can be mimicked by a suitable hybrid logic $\mathcal{H}$ (@)-formula [84] as exhibited in Fig. 25.

\subsubsection{Encoding domain sort expressions}

The logic $\mathcal{M}\left(E_{n}\right)$ is not equipped with syntactic constructs for naming worlds such as nominals in hybrid logic $\mathcal{H}$ (@). However, due to the fact that the $\mathbf{E}_{>n}$-operators combine counting properties and global range, we are able to bypass explicit labelling expressions by exploiting the $E_{=1}$-operator in an appropriate way. For each input set of formulas $\Gamma$ we can encode domain sort expressions as specified in Fig. 26. We only need to show that the respective formulas in the figure actually encode the respective domain sort expressions.

Proposition 8.5. The domain sort expressions $v(\varphi, x), R(x, y), x \approx y$ hold in a first-order model $\mathfrak{A}$ iff the respective $\mathcal{M}\left(E_{n}\right)$-formulas from Fig. 26 hold in a suitable conservative extension $\mathfrak{M}^{\prime}$ of a Kripke model $\mathfrak{M}=\langle W, R, V\rangle$, where $W=\operatorname{Dom}(\mathfrak{A}), R^{\mathfrak{A}}=R^{\mathfrak{M}}$ and $v(p, x)$ iff $x \in V(p)$ for each $p \in$ PROP.

Proof. $v(\varphi, x), \neg v(\varphi, x) . \quad(\Leftarrow)$ Suppose that a formula $\varphi$ holds at a world $x$ (which is expressed by $v(\varphi, x)$ in $\mathfrak{A})$. Then we pick a conser- 


$$
\begin{aligned}
\operatorname{Tr}_{\mathcal{M}\left(\mathrm{E}_{n}\right)}(v(\varphi, x)) & =\mathrm{E}_{=1} p_{x} \wedge \mathrm{E}_{=0}\left(p_{x} \wedge \neg \varphi\right) \\
\operatorname{Tr}_{\mathcal{M}\left(\mathrm{E}_{n}\right)}(\neg v(\varphi, x)) & =\mathrm{E}_{=1} p_{x} \wedge \mathrm{E}_{=0}\left(p_{x} \wedge \varphi\right) \\
\operatorname{Tr}_{\mathcal{M}\left(\mathrm{E}_{n}\right)}(R(x, y)) & =\mathrm{E}_{=1} p_{x} \wedge \mathrm{E}_{=1} p_{y} \wedge \mathrm{E}_{=0}\left(p_{x} \wedge \neg \nabla p_{y}\right) \\
\operatorname{Tr}_{\mathcal{M}\left(\mathrm{E}_{n}\right)}(\neg R(x, y)) & =\mathrm{E}_{=1} p_{x} \wedge \mathrm{E}_{=1} p_{y} \wedge \mathrm{E}_{=0}\left(p_{x} \wedge \diamond p_{y}\right) \\
\operatorname{Tr}_{\mathcal{M}\left(\mathrm{E}_{n}\right)}(x \approx y) & =\mathrm{E}_{=1} p_{x} \wedge \mathrm{E}_{=1} p_{y} \wedge \mathrm{E}_{=0}\left(p_{x} \wedge \neg p_{y}\right) \\
\operatorname{Tr}_{\mathcal{M}\left(\mathrm{E}_{n}\right)}(x \neq y) & =\mathrm{E}_{=1} p_{x} \wedge \mathrm{E}_{=1} p_{y} \wedge \mathrm{E}_{=0}\left(p_{x} \wedge p_{y}\right)
\end{aligned}
$$

In each case $p_{x}, p_{y}$ are fresh propositional variables not occurring in $\Gamma$.

Figure 26: $\mathcal{M}\left(\mathrm{E}_{n}\right)$-translation function for domain-sort expressions.

vative extension of $\mathfrak{M}$ by introducing a fresh propositional variable $p_{x}$ and extending $V$ to $V^{\prime}$ in such a way that $V^{\prime}$ agrees with $V$ on all propositional variables from formulas under consideration and $V^{\prime}\left(p_{x}\right)=\{x\}$. Then, by definition of $\mathrm{E}_{=1}, \mathrm{E}_{=1} p_{x}$ holds and since $p_{x}$ holds at a unique world, namely $x$ where also $\varphi$ holds, for no world it is the case that both $p_{x}$ and $\neg \varphi$ hold. Therefore, $\mathrm{E}_{=1} p_{x} \wedge \mathrm{E}_{=0}\left(p_{x} \wedge \neg \varphi\right)$ is satisfied on $\mathfrak{M}^{\prime}$.

$(\Rightarrow)$ Suppose that a formula $\mathrm{E}_{=1} p_{x} \wedge \mathrm{E}_{=0}\left(p_{x} \wedge \neg \varphi\right)$ is satisfied on a conservative extension $\mathfrak{M}^{\prime}=\left\langle W, R, V^{\prime}\right\rangle$, where $v^{\prime}\left(p_{x}\right)=\{x\}$. It therefore follows that for no world both $p_{x}$ and $\neg \varphi$ hold. Since $x$ is the only world where $p_{i}$ holds, it must be the case that $\varphi$ holds in $x$. The case of $\neg v(\varphi, x)$ proceeds analogically.

$R(x, y), \neg R(x, y) . \quad(\Leftarrow)$ Suppose that $R(x, y)$ holds. We pick a conservative extension of $\mathfrak{M}$ by introducing two fresh propositional variables $p_{x}$ and $p_{y}$ and extending $V$ to $V^{\prime}$ such that they agree on all propositional variables from $\Gamma$ and, additionally, $V^{\prime}\left(p_{x}\right)=\{x\}$ and $V^{\prime}\left(p_{y}\right)=\{y\}$. Since $p_{y}$ holds in $y$ and $(x, y) \in R$, then by Definition 5.2 we obtain that $\nabla p_{y}$ holds in $x . x$ is the unique world where $p_{x}$ holds so for all worlds it cannot be the case that $p_{x} \wedge \neg \diamond p_{y}$ holds. Hence the conclusion.

$(\Rightarrow)$ Assume that $\mathrm{E}_{=1} p_{x} \wedge \mathrm{E}_{=1} p_{y} \wedge \mathrm{E}_{=0}\left(p_{x} \wedge \neg \nabla p_{y}\right)$ is satisfied in a conservative extension of $\mathfrak{M}$ where $V^{\prime}\left(p_{x}\right)=\{x\}$ and $V^{\prime}\left(p_{y}\right)=\{y\}$. For no world in which $p_{x}$ is true $\neg \nabla p_{y}$ is also true. It follows that 
$\nabla p_{y}$ holds in $x$ and, by Definition 5.2, $R(x, y)$. We proceed similarly for $\neg R(x, y)$.

$x \approx y, x \neq y . \quad(\Leftarrow)$ Suppose that $x \approx y$ holds. It means that worlds $x$ and $y$ coincide on $\mathfrak{M}$. We pick a conservative extension of $\mathfrak{M}$ by adding two fresh propositional variables $p_{x}$ and $p_{y}$ and extending $V$ to $V^{\prime}$ in such way that $V^{\prime}\left(p_{x}\right)=\{x\}$ and $V^{\prime}\left(p_{y}\right)=\{y\}$. Since $x$ and $y$ are the same world which is the unique world where either, $p_{x}$ and $p_{y}$ hold, it means that there is no world in which $p_{x} \wedge \neg p_{y}$ hold. It completes this part of the proof.

$(\Rightarrow)$ Suppose that $\mathrm{E}_{=1} p_{x} \wedge \mathrm{E}_{=1} p_{y} \wedge \mathrm{E}_{=0}\left(p_{x} \wedge \neg p_{y}\right)$ is satisfied on $\mathfrak{M}^{\prime}$, a conservative extension of $\mathfrak{M}$ where $p_{x}, p_{y}$ are fresh and $V^{\prime}\left(p_{x}\right)=\{x\}, V^{\prime}\left(p_{y}\right)=\{y\}$. Since there is no world where both $p_{x}$ and $\neg p_{y}$ hold, it must be the case that in all worlds in which $p_{x}$ holds, $p_{y}$ also holds. But there is only one world satisfying $p_{x}$, namely $x$, and one world satisfying $y$, namely $y$. Since conjunction of both these hold in both worlds, by the definition of $\Vdash$ they must coincide.

We conduct a similar proof for $x \neq y$.

For convenience and economy of space, we introduce a new colon notation. We abbreviate formulas of the form: $E_{=1} \varphi \wedge E_{=0}(\varphi \wedge \neg \psi)$ as $\varphi: \psi$.

To accomplish the refinement of our calculus, we need to introduce one additional element. We define a countable set $\mathcal{F}=\left\{f_{j}\right\}_{j \in \mathbb{N}}$ where each $f_{j}:$ FORM $\times$ FORM $\rightarrow$ FORM is a function. The intention is to have a countable set of function symbols to obtain an expression class analogous to a class of Skolem terms in the first-order meta-language.

\subsubsection{Tableau calculus $\mathcal{T}_{\mathcal{M}\left(\mathrm{E}_{n}\right)}^{i}$}

Figure 27 presents the rules of the internalized calculus $\mathcal{T}_{\mathcal{M}\left(E_{n}\right)}^{i n-}$ cluding the unrestricted blocking mechanism. A benefit resulting from dispensing with domain symbols is fewer rules.

The colon notation makes the refined tableau calculus resemble standard internalized calculi for hybrid logics. For a given input formula $\varphi$ a label of the initial node is a propositional variable obtained by translating $v(\varphi, x)$ based on the encoding in Fig. 26. New labels introduced 
Rules for connectives:

$$
\begin{aligned}
& (\neg \neg) \frac{\varphi: \neg \neg \psi}{\varphi: \psi} \quad(\wedge) \frac{\varphi: \psi \wedge \chi}{\varphi: \psi, \varphi: \chi} \quad(\neg \wedge) \frac{\varphi: \neg(\psi \wedge \chi)}{\varphi: \neg \psi \mid \varphi: \neg \chi} \\
& (\diamond) \frac{\varphi: \diamond \psi}{\varphi: \diamond f(\diamond \psi, \varphi), f(\diamond \psi, \varphi): \psi} \quad(\neg \diamond) \frac{\varphi: \neg \nabla \psi, \varphi: \diamond \chi, \chi: \chi}{\varphi: \neg \psi} \\
& \left(\mathrm{E}_{>n}\right) \frac{\varphi: \mathrm{E}_{>n} \psi}{f_{1}\left(\mathrm{E}_{>n} \psi, \varphi\right): \psi, \ldots, f_{n+1}\left(\mathrm{E}_{>n} \psi, \varphi\right): \psi_{0<k<i \leq n+1} f_{k}\left(\mathrm{E}_{>n} \psi, \varphi\right): \neg f_{l}\left(\mathrm{E}_{>n} \psi, \varphi\right)} \\
& \left(\neg \mathrm{E}_{>n}\right) \frac{\varphi: \neg \mathrm{E}_{>n} \psi, \chi_{1}: \chi_{1}, \ldots, \chi_{n+1}: \chi_{n+1}}{\chi_{1}: \neg \psi|\cdots| \chi_{n+1}: \neg \psi||_{0<k<l \leq n+1} \chi_{k}: \neg \chi_{l}}
\end{aligned}
$$

Rules for equality, closure rule, unrestricted blocking rule:

$$
\begin{array}{lll}
\text { (ref:) } \frac{\varphi: \psi}{\varphi: \varphi} \quad \text { (con) } \frac{\varphi: \psi, \psi: \psi}{f(\chi, \varphi): f(\chi, \psi)} & \text { (sub1) } \frac{\varphi: \psi, \psi: \psi, \varphi: \chi}{\psi: \chi} \\
(\perp) \frac{\varphi: \psi, \varphi: \neg \psi}{\perp} & \text { (ub) } \frac{\varphi: \varphi, \psi: \psi}{\varphi: \psi \mid \varphi: \neg \psi} & \text { (sub2) } \frac{\varphi: \psi, \psi: \psi, \chi: \theta}{\chi: \theta[\psi / \varphi]}
\end{array}
$$

Rules for frame conditions:

$$
\begin{aligned}
& \text { (ref) } \frac{\varphi: \varphi}{\varphi: \diamond \varphi} \quad(\text { sym }) \frac{\varphi: \diamond \psi}{\psi: \diamond \varphi} \quad(\text { tran }) \frac{\varphi: \diamond \psi, \psi: \diamond \chi}{\varphi: \diamond \chi} \\
& \text { (ser) } \frac{\varphi: \varphi}{\varphi: \diamond f(\varphi: \varphi, \varphi)} \quad\left(\text { Eucl) } \frac{\varphi: \diamond \psi, \varphi: \diamond \chi}{\psi: \diamond \chi}\right.
\end{aligned}
$$

Figure 27: Rules for the calculus $\mathcal{T}_{\mathcal{M}\left(\mathrm{E}_{n}\right)}^{i}$. 
by the rules $(\diamond)$ and $\left(E_{>n}\right)$ are arbitrary formulas (by definition of functional symbols $\left.f_{i}\right)$. The reader might be surprised by the rule $(\diamond)$, since the first formula in the conclusion is of the same form as the premise formula, which might in principle lead to infinite derivations. What distinguishes these two is the fact that the formula under the scope of $\diamond$ in the premise is not necessarily a labelling formula, whereas a formula that appears under the scope of $\diamond$ in the conclusion certainly is. This makes it subjected to applications of the (sub1), (sub2) and (ub) rules which ensure finiteness (with respect to $(\diamond)$-application) of at least one of the branches.

The refinement consisting in refining away domain sort symbols, as described, preserves soundness, constructive completeness and termination. In order to conduct a proof of completeness, we define a "branch induced model" $\mathfrak{M}(\mathcal{B})=\langle\mathrm{W}, \mathrm{R}, \mathrm{V}\rangle$ as follows. Let $\varphi \sim_{\mathcal{B}} \psi$ iff $\varphi: \psi, \psi:$ $\psi \in \mathcal{B}$. Then:

$\mathrm{W}=\left\{[\varphi]_{\sim_{\mathcal{B}}} \mid \varphi: \varphi \in \mathcal{B}\right\}$,

$\mathrm{R}=\left\{\left\langle[\varphi]_{\sim_{\mathcal{B}}}[\psi]_{\sim_{\mathcal{B}}}\right\rangle \mid \varphi: \diamond \psi, \psi: \psi \in \mathcal{B}\right\}$

$\mathrm{V}=\left\{(p, U) \mid p \in\right.$ PROP,$p$ occurs on $\mathcal{B}$ and $\left.U=\left\{[\varphi]_{\sim_{\mathcal{B}}} \mid \varphi: p ß \in \mathcal{B}\right\}\right\}$.

\subsection{MetTEL2 implementation}

In order to implement a prover based on the prefix tableau calculus $\mathcal{T}_{\mathcal{M}\left(\mathrm{E}_{n}\right)}^{p}$ we used METTER $[90,1]$, a new tableau prover generator. METTEL automatically generates JAVA code of a tableau prover from the syntax specification of a logical theory and a set of tableau rules provided by the user. METTEL fully supports dynamic backtracking and backjumping and uses the unrestricted blocking mechanism to ensure termination. At the moment, METTEL does not support specifications of logical connectives and tableau rules which are parameterized by numerical values. Because of this, all the operators $\mathbf{E}_{>n}$ for different $n$ are specified as separate connectives up to some fixed number $m$. For each particular number $m$ required for the formalisation of a set of test problems, we wrote a script to automatically produce a language specification and a tableau specification for the logic $\mathcal{M}\left(E_{n}\right)$, where the parameter $n$ in the operators $E_{>n}$ is limited by $m$. The obtained 
language and tableau specifications are passed to METTER which generates a tableau prover for the logic of the restricted language. The generated prover is not intended to be a state-of-the-art prover but can be used for experimenting with the tableau calculus. During our investigation we found it useful to test problems and experiment with different rule refinements. For example, experimenting with the $\left(\neg \mathrm{E}_{>n}\right)$ rule and its refined variant we discovered that the calculus loses completeness under such a refinement. This directed us to look for a counterexample to the condition $(t)$ and to realize subsequently that this rule is not refinable. Various further experiments showed that it is possible to eliminate nominals from the calculus replacing them by formulas without loosing good properties of the calculus. The generated provers can be optimised and tuned further by integrating user implemented proof strategies using the of provided API of METTEL .

A specifications of the syntax of $\mathcal{M}\left(E_{n}\right)$ and the presented tableau calculus are available at the METTEL on-line demo page [1], where the user is allowed to amend the specifications and to regenerate the prover.

\subsection{OTHER EXISTING DECISION PROCEDURES}

Several decision procedures for logics with counting operators have been established so far, all of them in the field of description logics. Since, contrary to hybrid logics, there is no systematic survey on different decision procedures for modal (description) logics with global counting operators, the following section aims at providing a short, preliminary version of such a survey. In the remainder of the chapter, we therefore mention three tableau-based decision procedures to draw some parallels between them and our system.

\subsubsection{Analytic tableau calculus with pattern-based blocking}

Kaminski, Schneider and Smolka in $[59,60]$ introduce a tableau algorithm for the logic $\mathcal{S H O Q}$ (a description logic with nominals, graded modalities and global counting modalities). They also extend their construction on the logic with reflexive and transitive accessibility relations. 
We provide a brief description of their system and explain all necessary technical notions introduced on the way. Since the original paper of Kaminski et al. concerns the description logic $\mathcal{S H O Q}$, the notation used by them differs significantly from the standard notation from modal logic that has been used so far in this book. We therefore appropriately adapt the notation from [60] when necessary, not interfering with the content. Furthermore, the logic $\mathcal{S H O Q}$ is multi-modal, whereas the logic $\mathcal{M}\left(E_{n}\right)$ is uni-modal. Consequently, we skip the description of all those parts of Kaminski's et al. system, in which multi-modality is intrinsically involved (e.g. the part concerning role inclusion). All theorems and definitions in the following section come from [60].

In $[59,60]$, the authors assign to each expression a type. They distinguish two basic types: $B$ (from Boolean), whose interpretation is a two-element set including truth values: true and false, and $S$ (from states), whose range is a set of all worlds in a model. Let Nom be a set of nominals (which explicitly belong to the language of the logic, unlike in the case of the logic $\mathcal{M}\left(E_{n}\right)$ ), PROP be a set of propositional variables and let $R$ denote the accessibility relation. Then the types are ascribed to expressions in the following way:

$$
\begin{aligned}
i & : S, \quad \text { for all } i \in \text { NOM, } \\
p & : B, \quad \text { for all } p \in \text { PROP, } \\
R & : S S B, \\
\top, \perp & : B, \\
\neg & : B B, \\
\vee, \wedge & : B B B, \\
\approx & : S S B,
\end{aligned}
$$

The language of the logic is defined in its negation-normal form ${ }^{2}$ :

$$
\text { FORM }:=p|\neg p| i|\neg i| \varphi \wedge \psi|\varphi \vee \psi| \nabla \varphi|\square \varphi| \mathrm{E}_{>n} \varphi \mid \mathrm{E}_{<n} \varphi,
$$

where $i \in$ NOM, $p \in \operatorname{PROP}$ and $\varphi, \psi \in$ FORM.

2 The negation-normal form of a formula $\varphi$ is a formula $\psi$ such that $\varphi \equiv \psi$ and the negation sign $\neg$ only occurs in $\psi$ in front of propositional variables or nominals. 
On the other side, we define a set EXP which includes expressions occurring in a tableau derivation:

$$
\operatorname{EXP}::=\varphi i|\operatorname{Rij}| i \approx j|i \neq j| \perp,
$$

where $i \in \operatorname{NOM}$ and $\varphi, \psi \in$ FORM.

Clearly, $R, \approx, \neq$ belong to the meta-language rather than to the object language of the logic. In what follows, we can regard the calculus from [60] as a prefixed calculus. It is justified despite a twofold character of nominals. On the one hand, they are a part of the object language; on the other hand, they are exploited as labels in tableau expressions.

In the approach presented, a tableau is a derivation tree. Its nodes can be identified with sets of formulas from EXP that have been thus far introduced to a tableau by applications of tableau rules. A branch $\mathcal{B}$ is a set of formulas from EXP, which is a union of all nodes from a leaf to the root of a tree. Subsequent nodes therefore cumulate formulas from preceding ones. Since subsequent nodes are supersets of all their predecessors, we call the calculus cumulative - once a formula is introduced to a branch, it is never discarded. We can therefore identify a branch with its leaf.

A model for the logic is defined in an analogous manner to Definition 5.2. Tableau rules presented in Fig. 28 resemble the calculus $\mathcal{T}_{\mathcal{M}\left(\mathrm{E}_{n}\right)}$, however several significant differences appear, which are discussed more thoroughly in the ensuing part of the chapter.

The rules for the connectives are rather clear, however their side conditions require more thorough explanation. To provide such an explanation, we need to define the notions of equational closure, evidence and quasi-evidence.

By equational closure $\tilde{\mathcal{B}}$ of a branch $\mathcal{B}$ we denote a set of the form:

$$
\begin{aligned}
\tilde{\mathcal{B}}=\mathcal{B} & \cup\left\{\varphi i \mid \varphi \in \text { FORM } \wedge \exists j i \sim_{\mathcal{B}} j \wedge \varphi j \in \mathcal{B}\right\} \\
& \cup\left\{\operatorname{Rel}(i, j) \in\{R, \approx, \neq\} \mid \exists k, l i \sim_{\mathcal{B}} k\right. \\
& \left.\wedge j \sim_{\mathcal{B}} l \wedge \operatorname{Rel}(k, l) \in \mathcal{B}\right\} .
\end{aligned}
$$

Boldly speaking, if two labels (nominals) on a branch $\mathcal{B}$ are merged during a derivation and, as such, form the same equivalence class, the 
equational closure of $\mathcal{B}$ propagates all formulas and accessibility links satisfied by a label to all labels from the same equivalence class.

We now give the following definition:

DEFINITION 8.3 ([60]). A branch $\mathcal{B}$ is called evident if it satisfies all of the following conditions

$$
\begin{aligned}
& (\varphi \wedge \psi) i \in \mathcal{B} \quad \text { then } \quad \varphi i \in \tilde{\mathcal{B}} \wedge \psi i \in \tilde{\mathcal{B}}, \\
& (\varphi \vee \psi) i \in \mathcal{B} \quad \text { then } \quad \varphi i \in \tilde{\mathcal{B}} \vee \psi i \in \tilde{\mathcal{B}} \text {, } \\
& \diamond \varphi i \in \mathcal{B} \quad \text { then } \quad \exists j(R i j \in \tilde{\mathcal{B}} \wedge \varphi j \in \tilde{\mathcal{B}}), \\
& \square \varphi i \in \mathcal{B} \quad \text { then } \quad \forall j(R i j \in \tilde{\mathcal{B}} \Rightarrow \varphi j \in \tilde{\mathcal{B}}) \text {, } \\
& \mathrm{E}_{>n} \varphi i \in \mathcal{B} \quad \text { then } \operatorname{Card}\left(\left\{[j]_{\sim_{\mathcal{B}}} \mid \varphi j \in \tilde{\mathcal{B}}\right\}\right)>n \text {, } \\
& \mathrm{E}_{<n} \varphi i \in \mathcal{B} \quad \text { then } \operatorname{Card}\left(\left\{[j]_{\sim_{\mathcal{B}}} \mid \varphi j \in \tilde{\mathcal{B}}\right\}\right)<n \text {, } \\
& j i \in \mathcal{B} \quad \text { then } i \sim_{\mathcal{B}} j, \\
& \neg j i \in \mathcal{B} \text { then } i \psi_{\mathcal{B}} j \\
& j \neq i \in \mathcal{B} \text { then } i \psi_{\mathcal{B}} j \text {, } \\
& \neg p i \in \mathcal{B} \text { then } p i \notin \tilde{\mathcal{B}} \text {. }
\end{aligned}
$$

A formula $\varphi$ is called evident if it satisfies the right-hand side condition of the evidence definition

Intuitively, an evident branch contains a full description of a model that is to be constructed of it. Apparently, our notion of constructive completeness coincides with the notion of evidence in such a way that a calculus is constructively complete if for all tableaux every open and fully expanded branch is evident.

Now, the rules $(\Lambda)$ and $(\vee)$ are standard. To grasp the idea of the side condition for the rule $(\diamond)$ we need a definition of the notion of quasi-evidence, which we provide in the part concerning blocking. Before we apply the rule $(\square)$ we need to search through the equational closure of a branch $\mathcal{B}$ to verify whether a label under consideration has any $R$-successors. Obviously, a candidate for a successor must be already present on a branch. The rule $\left(E_{>n}\right)$ can only be applied provided that it is not already evident on a branch, i.e. there are no $n+1$ distinct worlds satisfying a formula in the scope of $E_{>n}$. This means that even if there exist $n$ worlds satisfying this formula, $\left(E_{>n}\right)$ will anyway introduce another $n+1$ fresh labels to a branch. The rule 
Rules for the connectives:
$(\wedge) \frac{(\varphi \wedge \psi) i}{\varphi i, \psi i}$
$(\vee) \frac{(\varphi \vee \psi) i}{\varphi i \mid \psi i}$
$(\diamond) \frac{\diamond \varphi i}{R i j, \varphi j}, j$ fresh, $\diamond \varphi i$ not quasi-evident on a branch $\mathcal{B}$
( $\square) \frac{\square \varphi i}{\varphi j}, j$ such that $R i j \in \tilde{\mathcal{B}}$

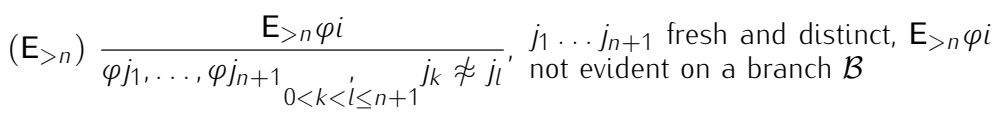

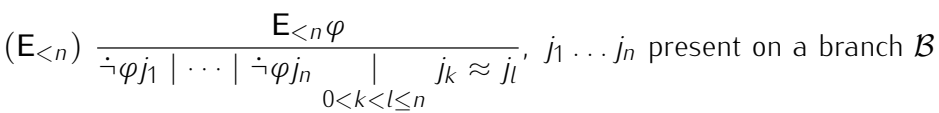

Rules for equality, closure rules:
(N) $\frac{j i}{j \approx i}$
(N) $\frac{\neg j i}{j \neq i}$
$\left(\perp_{\neg}\right) \frac{\neg p i}{\perp}, p i \in \tilde{\mathcal{B}}$
$\left(\perp_{\nsucc}\right) \frac{i \neq j}{\perp}, i \sim_{\mathcal{B}} j$

$\mathcal{B}$ stands for a branch on which a rule is applied.

$\tilde{\mathcal{B}}$ is the equational closure of a branch $\mathcal{B}$.

$\sim_{\mathcal{B}}$ is an equivalence relation over nominals on a branch $\mathcal{B}$, such that $i \sim_{\mathcal{B}} j$ iff $i \approx j \in \mathcal{B}$,

$\dot{\neg} \varphi$ stands for the negation-normal form of $\neg \varphi$.

Figure 28: Rules for the calculus $\mathcal{T}_{\mathcal{S H O Q}}$

$\left(\mathrm{E}_{<n}\right)$ works as follows: for each $n$-tuple of labels present on a branch it either merges two of them or it propagates to one of the labels the negation of a formula in the scope of $\mathrm{E}_{<n}$. Due to the universal range of $\mathrm{E}_{<n}$ we do not need to seek through the equational closure of a branch, since $\operatorname{lab}(\mathcal{B})=\operatorname{lab}(\tilde{\mathcal{B}})$, where $\operatorname{lab}(U)$ is a set of labels occurring in a set $U .(N)$ and $(\bar{N})$ are, in fact, rewriting rules that unify the notation within a branch and allow to apply the rule $\left(\perp_{\nsucc}\right)$. To adopt either of the closure rules we, again, need to examine the equational closure of a branch in search of possible inconsistencies.

It is apparent that the case of equality of labels within a branch in the aforementioned calculus is handled differently than in the calculus presented in Fig. 23. Whereas in our calculus we have several explicit rules responsible for propagating formulas, accessibility links 
and equalities among labels that have been merged during a derivation, in Kaminski's et al. calculus we need to compute and investigate the equational closure of a branch "on the fly", in parallel to the main derivation. We need to have the equational closure available at each stage of a derivation to be able to evaluate whether a particular rule is applicable to a formula.

Now we can formulate two important theorems:

THEOREM 8.6 (Soundness [60]). Let $\mathcal{B}_{1}, \ldots, \mathcal{B}_{n}$ be the branches obtained from a branch $\mathcal{B}$ by a rule of $\mathcal{T}_{\mathcal{S H O Q}}$. Then $\mathcal{B}$ is satisfiable iff there is some $i \in\{1, \ldots, n\}$ such that $\mathcal{B}_{i}$ is satisfiable.

THEOREM 8.7 (Evidence-completeness [60]). Every evident branch has a finite model.

Theorem 8.7 simultaneously establishes the finite model property for the logic $\mathcal{S H O \mathcal { Q }}$. However, even though the existence of a finite model of every evident branch is secured, the finiteness of each evident branch is not safe. In fact, if we leave the calculus as displayed in Fig. 28, it will lead to infinite derivations (and, respectively, infinite evident branches). A simple example of a formula generating such a derivation is $A(\nabla \varphi)$. What is interesting is that the example from Fig. 24 no longer applies as generating an infinite derivation, due to the side condition of the $\left(E_{<n}\right)$ rule, requiring for application the non-evidence of a formula.

To ensure the termination of the calculus, a blocking mechanism needs to be introduced. This mechanism is known as pattern-based blocking. First, we bring in the notion of a pattern. Let $\square(i)=$ $\{\square \varphi \mid \square \varphi i \in \mathcal{B}\}$ and $\diamond(i)=\{\diamond \varphi \mid \nabla \varphi i \in \mathcal{B}\}$.

Definition 8.4 (Pattern). Given a branch $\mathcal{B}$, by a pattern $P$ we understand a set of expressions of the form $\square \varphi$ or $\nabla \varphi$ occurring on $\mathcal{B}$. Without loss of generality, we assume that each pattern only contains one $\diamond$ formula. By $P_{\varphi}^{i}$ we denote a set $\{\nabla \varphi\} \cup \square(i)$ such that $\nabla \varphi \in \diamond(i)$. We call a pattern $P$ expanded if there exist labels $i$, j such that $P \subseteq P_{\varphi}^{i}$ for some $\varphi$, and $\varphi j, R i j \in \tilde{\mathcal{B}}$. We say that the label $i$ expands $P$ on $\mathcal{B}$.

A pattern is therefore a maximal set of modal formulas satisfied at a world that consistently generates a new world and propagates to it all formulas under the scope of modal operators. Clearly, for each label 
$i$ occurring on $\mathcal{B}$ there can be several distinct patterns $P^{i}$. At the same time, several distinct patterns might be expanded by the same label.

Now we are ready to explain the notion of quasi-evidence.

Definition 8.5 ([60]). A formula $\nabla \varphi i \in \mathcal{B}$ is quasi-evident on $\mathcal{B}$ if it is

1. either evident,

2. or has no successor on $\mathcal{B}$ and $P_{\varphi}^{i}$ is expanded on $\mathcal{B}$.

The rule $(\diamond)$ only applies to formulas that are not quasi-evident. It means that formulas satisfying the condition 2 from Definition 8.5 remain unexpanded, ipso facto breaking the evidence of a branch. However, we can easily complete a branch by just adding missing accessibility links. It is stated by the following:

THEOREM 8.8 (Evidence completion [60]). For every quasi-evident branch $\mathcal{B}$ there is an evident branch $\mathcal{B}^{\prime}$ such that $\mathcal{B} \subseteq \mathcal{B}^{\prime}$.

The pattern-based blocking mechanism, instead of generating new successor labels by application of the $(\diamond)$ rule, allows for searching among labels already present on a branch candidates for successors. If it finds one, it leaves a $\diamond$ formula unexpanded. Missing $R$-edges are added during the completion of a branch.

THEOREM 8.9 (Quasi-evidence [60]). Every open and fully expanded branch in $\mathcal{T}_{\mathcal{S H O Q}}$ is quasi-evident.

Theorems 8.7, 8.8 and 8.9 give us the completeness of the calculus.

As mentioned before, if we skip the quasi-evidence side condition from the rule $(\diamond)$, it can lead to infinite evident branches. On the contrary, quasi-evident branches are always finite. The following theorem establishes the termination of the calculus.

THEOREM 8.10 (Termination [60]). The size of every open and fully expanded branch $\mathcal{B}$ does not exceed $3 \cdot 2^{2 \mathcal{O}(\operatorname{Card}(\operatorname{Nom}(\varphi))+}$ $\operatorname{Card}(\operatorname{Sub}(\varphi)) \cdot 2^{\mathcal{O}(\operatorname{Card}(\operatorname{Nom}(\varphi))}$, where $\operatorname{NOM}(\varphi)$ is a set of all nominals occurring in the initial formula $\varphi$ and $\operatorname{Sub}(\varphi)$ is a set of all subformulas of $\varphi$.

Corollary 8.11. The calculus $\mathcal{T}_{\mathcal{S H O}} \mathcal{Q}$ runs in NEXPTIME. 
1.

2.

3.

4.

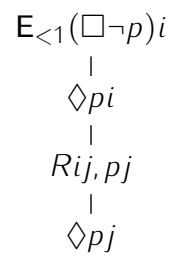

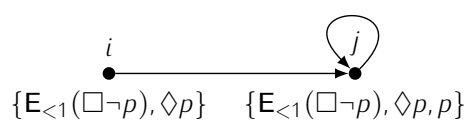

(b)

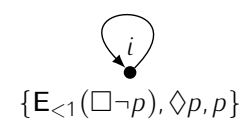

(c)

Figure 29: In (a) $\mathcal{T}_{\mathcal{S H O Q}}$-derivation of the formula $\mathrm{E}_{<1}(\square \neg p) i$ is displayed. Without loss of generality, we show at nodes of the derivation only those formulas that have been introduced by a preceding application of a rule. (b) presents a model generated from the branch in (a) (not minimal). In (c) we see a model generated by $\mathcal{T}_{\mathcal{M}\left(\mathrm{E}_{n}\right)}^{p}$ for the same formula (minimal).

The advantage of the pattern-based blocking mechanism lies in the fact that it does not lead to so many derivations as the unrestricted blocking which is a splitting rule. It means that the breadth of a tableau is larger in the case of $\mathcal{T}_{\mathcal{M}\left(\mathbf{E}_{n}\right)}^{p}$. However, if we take into account the length of the shortest open and fully expanded branch, $\mathcal{T}_{\mathcal{M}\left(\mathrm{E}_{n}\right)}^{p}$ is more efficient since it guarantees a generation of a minimal model, which $\mathcal{T}_{\mathcal{S H O Q}}$ does not. Figure 29 shows the difference in performance of both calculi.

A noticeable difference between $\mathcal{T}_{\mathcal{M}\left(\mathrm{E}_{n}\right)}^{p}$ and $\mathcal{T}_{\mathcal{S H O Q}}$ lies in the transparency of the rules. Whereas $\mathcal{T}_{\mathcal{M}\left(\mathrm{E}_{n}\right)}^{P}$ consists of explicit rules dealing with almost all aspects of a derivation (the only two side-conditions concern blocking applications of the rules $(\diamond)$ and $\left(E_{>n}\right)$ to labels that are not first in the appearance order), $\mathcal{T}_{\mathcal{S H O Q}}$ involves side-conditions that require constant examination of the equational closure of a branch. The profit brought by the second approach is, again, the restriction of the number of rule applications. For instance, in the case of the rule $\left(E_{>n}\right)$ the condition of the formula-evidence that blocks the application of the 
rule is weaker then the condition of non-precedence in the appearance order.

If we want to add some frame conditions, $\mathcal{T}_{\mathcal{M}\left(\mathrm{E}_{n}\right)}^{p}$ is definitely a more amenable calculus. We can freely restrict frame conditions, not jeopardizing the completeness. On the other hand, adding frame conditions to $\mathcal{T}_{\mathcal{S H O Q}}$ requires some serious modifications of the existing notions. We first need to extend the notion of evidence in a following way:

$$
\begin{aligned}
i \in \operatorname{NOM}(\mathcal{B}) & \text { then } & R i i \in \tilde{\mathcal{B}} \\
i, j, k & \in \operatorname{Nom}(\mathcal{B}) \quad \text { then } & R i j \in \tilde{\mathcal{B}} \wedge R j k \in \tilde{\mathcal{B}} \Rightarrow R i k \in \tilde{\mathcal{B}} .
\end{aligned}
$$

We notice that if we want to simply introduce an explicit rule for reflexivity:

$$
\overline{R i i}, i \in \operatorname{NOM}(\mathcal{B})
$$

it will lead to a trivial overlap of the notions of evidence and quasievidence, making the latter ineffective in blocking. To remedy it, we define a quasi-reflexive closure $R^{*}$ of the relation $R$ in the following way:

$$
R_{\mathcal{B}}^{*}=R \cup\left\{(i, j) \mid i, j \in \operatorname{NOM}(\mathcal{B}) \wedge i \sim_{\mathcal{B}} j\right\}
$$

Now we formulate the pre-evidence conditions. We do so by discarding the condition for reflexivity and modifying the conditions for $\diamond$ and $\square$ formulas, and transitivity as follows:

$$
\begin{array}{rll}
\nabla \varphi i \in \mathcal{B} & \text { then } & \exists j\left(R_{\mathcal{B}}^{*} i j \in \tilde{\mathcal{B}} \wedge \varphi j \in \tilde{\mathcal{B}}\right) \\
\square \varphi i \in \mathcal{B} & \text { then } & \forall j\left(R_{\mathcal{B}}^{*} i j \in \tilde{\mathcal{B}} \Rightarrow \varphi j \in \tilde{\mathcal{B}}\right) \\
i, j \in \operatorname{NOM}(\mathcal{B}) & \text { then } & \square \psi i \in \tilde{\mathcal{B}} \wedge R i j \in \tilde{\mathcal{B}} \Rightarrow \square \psi j \in \tilde{\mathcal{B}} .
\end{array}
$$

According to the foregoing, we re-construct the appropriate tableau rules:

$$
\begin{aligned}
& \text { ( } \square) \frac{\square \varphi i}{\varphi j}, j \text { such that } R^{*} i j \in \tilde{\mathcal{B}} \\
& (\text { Tran }) \frac{\square \varphi i}{\square \varphi j}, j \text { such that } R i j \in \tilde{\mathcal{B}}
\end{aligned}
$$

So equipped, we can establish the forthcoming theorems: 
THEOREM 8.12 (Evidence completion [60]). If $\mathcal{B}$ is pre-evident, then $\tilde{\mathcal{B}}$ is evident.

THEOREM 8.13 (Pre-evidence completion [60]). For every quasi-evident branch $\mathcal{B}$ there is a pre-evident branch $\mathcal{B}^{\prime}$ such that $\mathcal{B} \subseteq \mathcal{B}^{\prime}$.

Theorem 8.9 applies also to the calculus $\mathcal{T}_{\mathcal{S H} \mathcal{H} \mathcal{Q}}^{\text {Ref }}$, which, as a result, establishes the completeness. Termination as assured by the analog of Theorem 8.10.

\subsubsection{Constructive tableau calculus with pairwise blocking}

Numerous tableau calculi arose to deal with cardinality restrictions in such description logics as: $\mathcal{S H I Q ~}([44,45,49]), \mathcal{S R I} \mathcal{\mathcal { Q }}$ ([50]), SROIQ ([51]) and the most expressive and powerful logic $\mathcal{S H O I \mathcal { Q }}([46,47])^{3}$. Some of them, like $\mathcal{S R O I} \mathcal{Q}$ and $\mathcal{S H O I} \mathcal{L}$, as involving nominals, can be considered as notational variants of hybrid logics. The rest of the logics mentioned above lack the expressive power of hybrid logics (they neither contain nominals, nor global counting operators in their languages), however tableau calculi designed for these logics still remain in the area of our interest due to the way they deal with formulas containing cardinality constraints. All tableau algorithms from $[50,51,44,45,46,47,49]$ have a common core. They are based on the constructive approach to tableau calculi, which consists in, roughly speaking, defining a tableau not as a derivation tree, like in the analytical approach, but as an abstraction of a model, approximation of which is constructed by an algorithm.

A basis of the tableau calculus that we present below is the algorithm for the logic $\mathcal{S H \mathcal { I }}$ from $[44,45,49]$. The necessary modifications that we had to introduce to the algorithm concern global counting operators, which are not handled by the respective rules. The fact that no calculus within this approach deals with global counting modalities is not a coincidence. The reason for the poor algorithmic tractability of these modalities will become clear in the remainder of this section.

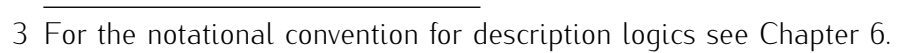


Like in the previous section, in presenting the tableau calculus we adapt the notation to make it coherent with the hybrid logic notation hitherto used.

We start from defining a closure of a formula $\varphi$. We assume all formulas to be in negation-normal form $(\operatorname{NNF})^{4}$. We abbreviate $\operatorname{NNF}(\neg \varphi)$ as $\dot{\neg} \varphi$.

$$
\operatorname{Clos}(\varphi)=\operatorname{Sub}(\varphi) \cup\{\dot{\neg} \psi \mid \psi \in \operatorname{Sub}(\varphi)\},
$$

where $\operatorname{Sub}(\varphi)$ is a set of all subformulas of $\varphi$. We therefore define the set Fclos as follows:

$\operatorname{Fclos}(\varphi)=\operatorname{Clos}(\varphi) \cup\left\{\square_{\mathcal{A}_{i}(q)} \psi \mid \square_{i} \psi \in \operatorname{Clos}(\varphi)\right.$ and $\mathcal{A}_{i}$ has a state $\left.q\right\}$, where $\mathcal{A}_{i}$ is a non-deterministic finite automaton associated with the accessibility relation $R_{i}$. Before we explain the notion of a non-deterministic finite automaton introduced in the foregoing definition, and its relevance to our considerations, we first provide the definition of a tableau.

Definition 8.6. $\mathrm{T}=\langle\mathrm{S}, \mathcal{L}, \mathcal{E}\rangle$ is a tableau for a formula $\varphi$ if, and only if:

$\mathrm{S}$ is a non-empty set,

$\mathcal{L}: \mathrm{S} \longrightarrow 2^{\mathrm{Fclos}(\varphi)}$ maps each element to a set of formulas,

$\mathcal{E}: \mathrm{R} \longrightarrow 2^{\mathrm{S} \times \mathrm{S}}$ maps each accessibility relation to a set of pairs of elements in $\mathbf{S}$.

Additionally, for all $s, t \in \mathrm{S}, \psi_{1}, \psi_{1}, \psi_{2} \in \operatorname{Fclos}(\varphi)$ and $R_{i} \in \mathrm{R}, \mathrm{T}$ satisfies the following conditions:

(P0) there is some $s \in \mathrm{S}$ with $\varphi \in \mathcal{L}(s)$,

(P1) if $\psi \in \mathcal{L}(s)$, then $\dot{\neg} \psi \notin \mathcal{L}(S)$,

(P2) if $\psi_{1} \wedge \psi_{2} \in \mathcal{L}(s)$, then $\psi_{1} \in \mathcal{L}(s)$ and $\psi_{2} \in \mathcal{L}(s)$,

(P3) if $\psi_{1} \vee \psi_{2} \in \mathcal{L}(s)$, then $\psi_{1} \in \mathcal{L}(s)$ or $\psi_{2} \in \mathcal{L}(s)$,

(P4a) if $\square_{\mathcal{A}_{i}(p)} \psi \in \mathcal{L}(s),\langle s, t\rangle \in \mathcal{E}\left(R_{i}\right)$, and $p \stackrel{R_{i}}{\longrightarrow} q \in \mathcal{A}_{i}(p)$, then $\square_{\mathcal{A}_{i}(q)} \psi \in \mathcal{L}(t)$,

$4 \overline{\text { That is the reason for introducing }} \vee$ instead of $\neg \wedge$ and $\square$ instead of $\neg \diamond$. 
(P4b) if $\square_{\mathcal{A}_{i}} \psi \in \mathcal{L}(s)$ and $\epsilon \in L\left(\mathcal{A}_{i}\right)^{5}$, then $\psi \in \mathcal{L}(t)$,

(P5) if $\nabla_{i} \psi \in \mathcal{L}(s)$, then there exists $t$ such that $\langle s, t\rangle \in \mathcal{E}\left(R_{i}\right)$ and $\psi \in \mathcal{L}(t)$,

(P6) if $\square_{i} \psi \in \mathcal{L}(S)$, then $\square_{\mathcal{A}_{i}} \psi \in \mathcal{L}(s)$,

(P7) if $\mathrm{E}_{>n} \psi \in \mathcal{L}(s)$, then $\operatorname{Card}(\{s \mid \psi \in \mathcal{L}(s)\})>n$,

(P9) if $\mathrm{E}_{<n} \psi \in \mathcal{L}(s)$, then $\operatorname{Card}(\{s \mid \psi \in \mathcal{L}(s)\})<n$,

(P10) if $\mathrm{E}_{<n} \psi \in \mathcal{L}(s)$, then $\psi \in \mathcal{L}(t)$ or $\neg \psi \in \mathcal{L}(t)$ for all $t \in \mathrm{S}$.

As we can see, a tableau is an abstraction of a model in a sense that a node $s \in \mathrm{S}$ does not need to contain a full description of itself, even with respect only to the signature consisting of propositional variables of an initial formula $\varphi$. Besides, as it will turn out soon, when we proceed to characterising additional accessibility relation conditions, a tableau does not have to provide a full, explicit information about a labelling of each edge $\langle s, t\rangle \in \mathrm{S}$.

First, however, we give a brief explication of a role played by nondeterministic finite automata introduced in definitions of Fclos and a tableau. Since we deal with a uni-modal logic with only one accessibility relation $R$ this exertion might appear superfluous. Nevertheless, let's imagine that we would like to introduce more accessibility relations $R_{1}, \ldots, R_{n}$ associated with respective modalities $\square_{1}, \ldots, \square_{n}$. We might also wish to impose some specific conditions on relations among $R_{1}, \ldots, R_{n}$.

EXAMPLE 8.1. For instance, let $R_{k}$ be a "knowledge" accessibility relation and let $R_{b}$ stand for a "belief" accessibility relation. The following inclusions express mutual relations between $R_{k}, R_{b}$ and their compositions:

1. $R_{k} \subseteq R_{b}$,

2. $R_{k} \circ R_{b} \subseteq R_{b}$,

3. $R_{b} \circ R_{k} \subseteq R_{b}$,

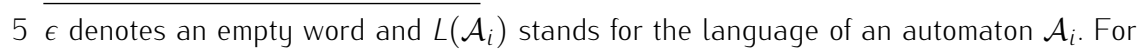
details see the remainder of the section. 
4. $R_{k} \circ R_{k} \subseteq R_{k}$

5. $R_{k}^{-} \subseteq R_{k}$.

We can intuitively understand the foregoing inclusions as follows:

1. "whenever a subject knows $x$, she also believes $x$ ",

2. "whenever a subject knows that she believes $x$, she believes $x$ ",

3. "whenever a subject believes that she knows $x$, she believes $x$ ",

4. "whenever a subject knows that she knows $x$, she knows $x$ ",

5. "a subject would know what is the case in the current world, if she found herself in a world that is knowledge-accessible to her" 6 .

Even if we want to keep a logic uni-modal, we still can take advantage of such inclusions in defining particular properties of an accessibility relation. For instance, a relation $R$ is transitive if, and only if it satisfies the condition:

$$
R \circ R \subseteq R
$$

Symmetry is given by the following:

$$
R^{-} \subseteq R
$$

where an inverse relation $R^{-}$is defined as the set $\{\langle x, y\rangle \mid\langle y, x\rangle \in R\}$. For Euclideaness of $R$ we fix:

$$
R^{-} \circ R \subseteq R
$$

This is a more abstract way of coping with properties of accessibility relations than introducing subsequent explicit conditions to the definition of a tableau:

(P11) if $\operatorname{Tran}\left(R_{i}\right),\langle s, t\rangle \in \mathcal{E}\left(R_{i}\right)$ and $\langle t, u\rangle \in \mathcal{E}\left(R_{i}\right)$, then $\langle s, u\rangle \in$ $\mathcal{E}\left(R_{i}\right)$

6 The presented, rather trivial, example only serves to illustrate how we can predefine mutual interferences between accessibility relations involved in a modal logic fashion (in Hilbert-style systems we can express them with suitable axioms). In description logics such a set of relation subsumptions is one of the basic tools known under the name of role hierarchy. 
(P12) if $\operatorname{Sym}\left(R_{i}\right),\langle s, t\rangle \in \mathcal{E}\left(R_{i}\right)$, then $\langle t, s\rangle \in \mathcal{E}\left(R_{i}\right)$,

(P13) if $\operatorname{Eucl}\left(R_{i}\right),\langle s, t\rangle \in \mathcal{E}\left(R_{i}\right)$ and $\langle s, u\rangle \in \mathcal{E}\left(R_{i}\right)$, then $\langle t, u\rangle \in$ $\mathcal{E}\left(R_{i}\right)$.

Unfortunately, there exists no inclusion of a desirable kind that would express reflexivity or irreflexivity. What follows, it is necessary to formulate and introduce explicit tableau conditions:

(P14) if $\operatorname{Ref}\left(R_{i}\right)$ and $s \in \mathrm{S}$, than $\langle s, s\rangle \in \mathcal{E}\left(R_{i}\right)$,

(P15) if $\operatorname{Irr}\left(R_{i}\right)$ and $s \in \mathrm{S}$, than $\langle s, s\rangle \notin \mathcal{E}\left(R_{i}\right)$.

Still, it is possible to handle accessibility relations' inclusions (and, consequently, certain properties of these relations as special cases of such inclusions) more abstractly. For this purpose, we engage nondeterministic finite automata (NFA) associated with accessibility relations. Intuitively, a NFA $\mathcal{A}_{i}$ ascribed to a relation $R_{i}$ "keeps track" of all (compositions of) relations subsumed by $R_{i}$. In the forthcoming part of the section, we briefly describe the construction of such an automaton and illustrate how it works in reference to Example 8.1.

Following [44], the construction of a NFA $\mathcal{A}_{R}$ assigned to a relation $R$ proceeds as below:

FIRST, we construct a non-deterministic finite pre-automaton $\mathcal{P}_{R}$ consisting of two distinct states: an initial state $i_{R}$ and a final state $f_{R}$ linked by a transition $\stackrel{R}{\longrightarrow}$. Now for each composition $S=R_{1} \circ \cdots \circ R_{n}$ of accessibility relations, such that $S \subseteq R$, we determine:

1. if $S=R \circ R$, then $\mathcal{P}_{R}$ contains $f_{R} \stackrel{R}{\longrightarrow} i_{R}$,

2. if $S=R_{1} \circ \cdots \circ R_{n}$ and $R_{1} \neq R \neq R_{n}$, then $\mathcal{P}_{R}$ contains

$$
i_{R} \stackrel{\epsilon}{\longrightarrow} i_{S} \stackrel{R_{1}}{\longrightarrow} f_{S}^{1} \stackrel{R_{2}}{\longrightarrow} \ldots \stackrel{R_{n}}{\longrightarrow} f_{S}^{n} \stackrel{\epsilon}{\longrightarrow} f_{R}
$$

3. if $S=R \circ R_{2} \circ \cdots \circ R_{n}$, then $\mathcal{P}_{R}$ contains

$$
f_{R} \stackrel{\epsilon}{\longrightarrow} i_{S} \stackrel{R_{2}}{\longrightarrow} f_{S}^{2} \stackrel{R_{3}}{\longrightarrow} \ldots \stackrel{R_{n}}{\longrightarrow} f_{S}^{n} \stackrel{\epsilon}{\longrightarrow} f_{R},
$$

4. if $S=R_{1} \circ \cdots \circ R_{n-1} \circ R$, then $\mathcal{P}_{R}$ contains

$$
i_{R} \stackrel{\epsilon}{\longrightarrow} i_{S} \stackrel{R_{1}}{\longrightarrow} f_{S}^{1} \stackrel{R_{2}}{\longrightarrow} \ldots \stackrel{R_{n-1}}{\longrightarrow} f_{S}^{n-1} \stackrel{\epsilon}{\longrightarrow} i_{R} .
$$


As usual, $\epsilon$ denotes an empty expression. We also define a mirroredcopy of $\mathcal{P}_{R}$ to be able to cope with the $R^{-}$accessibility relation. A mirrored-copy $\operatorname{MC}\left(\mathcal{P}_{R}\right)$ of $\mathcal{P}_{R}$ is obtained by flipping transition arrows, replacing each label $R_{i}$ for $R_{i}^{-}$and switching initial and final states.

SECOND, we distinguish between two cases:

1. if $R$ is not symmetric, i.e., $R^{-} \nsubseteq R$, then $\hat{\mathcal{P}}_{R}=\mathcal{P}_{R}$,

2. if $R$ is symmetric, i.e., $R^{-} \subseteq R$, then $\hat{\mathcal{P}}_{R}=\mathcal{P}_{R} \uplus \operatorname{MC}\left(\mathcal{P}_{R}\right) \cup$ $\left\{i_{R} \stackrel{\epsilon}{\longrightarrow} f_{R}^{\prime}, i_{R}^{\prime} \stackrel{\epsilon}{\longrightarrow} f_{R}, f_{R} \stackrel{\epsilon}{\longrightarrow} i_{R}^{\prime}, f_{R}^{\prime} \stackrel{\epsilon}{\longrightarrow} i_{R}\right\}$, where $i_{R}, f_{R}, i_{R}^{\prime}, f_{R}^{\prime}$ are initial and final states of, respectively, $\mathcal{P}_{R}$ and $\operatorname{MC}\left(\mathcal{P}_{R}\right)$, and $\uplus$ is a disjoint union. We also set $i_{R}$ as the only initial and $f_{R}$ as the only final state of $\hat{\mathcal{P}}_{R}$.

THIRD, if $R$ does not occur on the right-hand side of any accessibility relation inclusions, we set $\mathcal{A}_{R}=\hat{\mathcal{P}}_{R}$. Otherwise, if there exists $S=$ $R_{1} \circ \cdots \circ R_{n}$ such that $S \subseteq R$, then for each $p \stackrel{R_{i}}{\rightarrow} q \in \hat{\mathcal{P}}_{R}$, such that $R_{i} \neq R$, we attach to $\hat{\mathcal{P}}_{R}$ a copy of $\mathcal{A}_{R_{i}}$ and the transitions $p \stackrel{\epsilon}{\longrightarrow} i_{R_{i}}$, $f_{R_{i}} \stackrel{\epsilon}{\longrightarrow} q$. Informally, we can say that by that means we recursively close $\hat{\mathcal{P}}_{R}$ under sub-automata.

By a language $L\left(\mathcal{A}_{R}\right)$ of an automaton $\mathcal{A}_{R}$ we denote a set of all expressions acceptable by this automaton, i.e. all expressions of the form $R_{1} R_{2} \ldots R_{n}$, such that $R_{1} \circ R_{2} \circ \cdots \circ R_{n} \subseteq R$. By a language $L\left(\mathcal{A}_{R}(p)\right)$ of an automaton $\mathcal{A}_{R}$ in a state $p$ we denote a set of all expressions that, as an input in $p$, lead to the final state of $\mathcal{A}_{R}$ along transition edges.

In Fig. 30 a step-by-step construction of an automaton for the relation $R_{b}$ from Example 8.1 is presented.

Now we can get back to Definition 8.6 and briefly comment on points (P4a), (P4b) and (P6). (P6) introduces a NFA associated with $R_{i}$, when a formula of the form $\square_{i} \varphi$ is involved. Thenceforth $\varphi$ is propagated according to transitions in $\mathcal{A}_{i}$ - a NFA which keeps track of all inclusions in which $R_{i}$ occurs on the right hand-side. Obviously, it is implicitly assumed that an introduced NFA is in its initial state. (P4a) captures the way transitions in an automaton are performed. It allows to propagate NFA $\square$ formulas along $R_{i}$ edges whenever a current state of an automaton contains an outgoing $R_{i}$ transition arrow. The NFA changes 


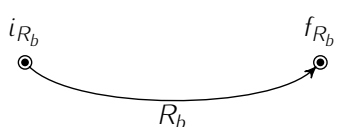

(a)

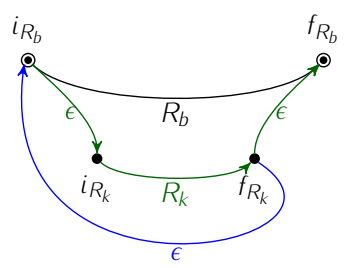

(c)

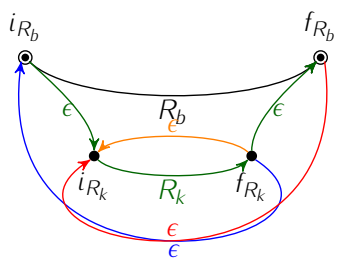

(e)

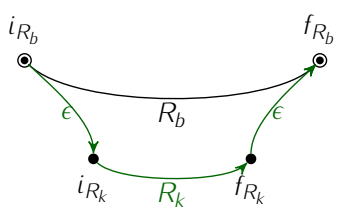

(b)

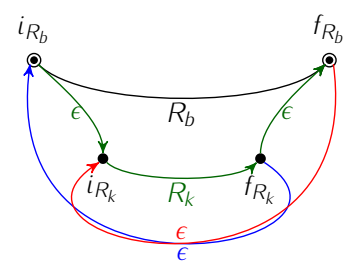

(d)

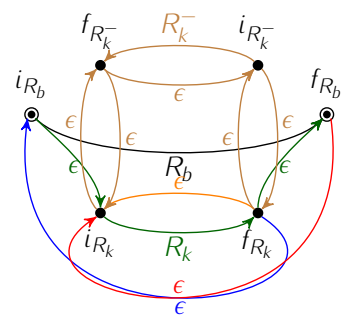

(f)

Figure 30: In the schema above, a step-by-step construction of an NFA $\mathcal{A}_{R_{b}}$ for the relation $R_{b}$ is displayed. In (a) we set the initial and the final state of the NFA and link them together using an $R_{b}$ transition arrow. In (b) we embed the subsumption of $R_{k}$ by $R_{b}$. In (c) and (d), the second and the third inclusions from Example 8.1 are complied according to the first point of the foregoing description of the construction of automata. In (e) and (f) we develop the $\mathcal{A}_{R_{k}}$ within $\mathcal{P}_{R_{b}}$. (e) shows how we handle the transitivity of $R_{k}$. In (f) we paste $\operatorname{MC}\left(\mathcal{P}_{R_{k}}\right)$ and link it to $\mathcal{P}_{R_{k}}$ within $\mathcal{P}_{R_{b}}$ a suitable way, thus taking care of the symmetry of $R_{k}$. 
its state to a state the transition arrow leads to. A box formula with the updated NFA is transferred to all $R_{i}$ successors of a current world. According to (P4b), if there is the $\epsilon$-transition arrow departing from the current state of the NFA and leading to its final state, a formula under the scope of $\square_{i}$ is expanded to a current world.

We are ready to formulate the following:

THEOREM 8.14 ([47]). A $\mathcal{M}\left(\mathrm{E}_{n}\right)$ formula $\varphi$ is satisfiable if, and only if there exists a tableau for $\varphi$, satisfying (P0)-(P10).

Constructing a tableau out of a model is rather trivial. It amounts to adding appropriate NFA $\square$ formulas to worlds that already contain ordinary $\square$ formulas. The reverse direction of the proof boils down to extending signatures of all worlds to $\operatorname{PROP}(\varphi)$ and adding all missing accessibility edges according to settings of transition edges in automata attached to $\square$ formulas in these worlds.

However, one can note that a tableau defined in this fashion does not have to be necessarily finite. It means that, in principle, if we want to directly construct an arbitrary tableau by means of a calculus, it might result in an infinite derivation. We need, therefore, a further abstraction of the notion of a model. This abstraction should approximate a tableau, but simultaneously it should be finite. We introduce such a concept and name it a completion graph.

Definition 8.7 (Completion graph). Let $\varphi$ be a $\mathcal{M}\left(\mathrm{E}_{n}\right)$-formula. A completion graph for $\varphi$ is a directed graph $G=\langle V, E, \mathcal{L}, \neq\rangle$ such that:

1. each node $v \in V$ is labelled with a set $\mathcal{L}(v) \subseteq \operatorname{Fclos}(\varphi)$,

2. each edge $\langle v, w\rangle \in E$ is labelled with a set of accessibility relations,

2. each inequality between nodes $v, w \in V$ is marked by a binary relation $\neq \subseteq V^{2}$.

If $\langle v, w\rangle \in E$ and $R \in \mathcal{L}(\langle v, w\rangle)$, then we call $w$ an $R$-successor of $v$, and $v$ is called an $R$-predecessor of $w^{7}$ a node belonging to the transitive closure of the $R$-successorship relation is called an $R$-descendant,

7 Note that as long as we consider only a uni-modal logic, we can confine ourselves to the notions of successor and predecessor, without any indices. 
whereas a node in the transitive closure of the $R$-predecessorship relation is an $R$-ancestor.

$W$ call a node $v$ blocked if it is directly or indirectly blocked. A node $v$ is directly blocked if none of its ancestors are blocked and $v$ has ancestors $v^{\prime}, w, w^{\prime}$ such that:

1. $v$ is a successor of $v^{\prime}$ and $w$ is a successor of $w^{\prime}$,

2. $\mathcal{L}(v)=\mathcal{L}(w)$ and $\mathcal{L}\left(v^{\prime}\right)=\mathcal{L}\left(w^{\prime}\right)$,

3. $\mathcal{L}(\langle w, v\rangle)=\mathcal{L}\left(\left\langle w^{\prime}, v^{\prime}\right\rangle\right)$.

A node $v$ is indirectly blocked if one of its ancestors is blocked.

We say that $G$ contains a clash if one of the following conditions is satisfied

1. $\perp \in \mathcal{L}(v)$ for some $v \in V$,

2. $\{\psi, \neg \psi\} \subseteq \mathcal{L}(v)$ for some $v \in V$ and $\psi \in \operatorname{Sub}(\varphi)$,

3. $\mathrm{E}_{<n} \psi \in \mathcal{L}(v)$ for some $v \in V$ and $\mathrm{E}_{<n} \psi \in \operatorname{Sub}(\varphi)$, and there exist $w_{1}, \ldots, w_{n} \in V$ such that $w_{i} \neq w_{j}$ and $\psi \in \mathcal{L}\left(w_{i}\right)$ for $1 \leq i<j \leq$ $n$.

Optionally, if we consider an irreflexive accessibility relation, we add the following:

4. $\langle v, v\rangle \in E$ and $R \in \mathcal{L}(\langle v, v\rangle)$ for some $R$ such that $\operatorname{Irr}(R)$.

A graph $G$ is called complete if no further rules are applicable to any of its nodes.

The foregoing definition of a completion graph introduces a new blocking mechanism, the so-called pairwise blocking. It evaluates pairs of nodes rather than single nodes in terms of occurring repetitions. Although in the case of the logic $\mathcal{M}\left(\mathrm{E}_{n}\right)$ pairwise blocking appears too powerful (the ordinary blocking for nodes suffices to ensure termination), it is suitable for termination of stronger logics involving nominals, graded modalities and graded inverse modalities. These logics do not enjoy the finite model property and the tree model property is almost completely lost [47], which can result in the loss of the completeness 
if we use particular blocking mechanisms for single nodes. ${ }^{8}$ An interesting feature of the pairwise blocking mechanism is its dynamic character. Nodes can get blocked, unblocked and blocked afresh during a derivation.

Figure 31 presents the rules of the calculus for $\mathcal{M}\left(E_{n}\right)$. The rules $(\wedge)$ and $(\vee)$ are rather straightforward. The rule $\left(\nabla_{i}\right)$ only introduces a new node to a graph if it is not the case that there already exists an $R_{i}$-successor satisfying a formula under the scope of $\nabla_{i}$. That prevents from creating superfluous vertices in a graph, which distinguishes the above calculus from $\mathcal{T}_{\mathcal{M}\left(\mathrm{E}_{n}\right)}$. ( $\square_{i}^{1}$ introduces to a node a NFA box formula, whenever an ordinary $\square$ formula is present in a node. ( $\left.\square_{i}^{2}\right)$ verifies whether a NFA indexing a $\square$ formula is in a state that enables performing a transition along the edge labelled by an accessibility relation whose edge outgoes from the current node. If that is the case, $\left(\square_{i}^{2}\right)$ performs the transition and propagates a suitably updated NFA $\square$ formula along the edge. $\left(\square_{i}^{3}\right)$ checks whether an automaton in a NFA box formula is in a state linked with a final state by an arrow labelled by the empty word $\epsilon$. If so, it propagates a formula under the scope of $\square \mathcal{A}_{i}$ to the current node. (ch) is a non-deterministic rule that accommodates the condition (P10) from Definition 8.6. $\left(E_{>n}\right)$ creates $n+1$ fresh and distinct nodes with a formula under the scope of $\mathbf{E}_{>n}$ in their labels, provided that such $n+1$-tuple is not already present in a graph. Note that if there exists no such tuple, the rule introduces all $n+1$ nodes, and not only $(n+1)-k$ nodes, where $k$ is the number of nodes containing a given formula and already present in the graph. Since $\left(E_{>n}\right)$ is already a rule scheme with $n$ as a parameter, if we want it to introduce the exact required number of nodes, for each $n$ we would need to set another formula scheme with $0 \leq k \leq n$ as a parameter. The non-deterministic rule $\left(\mathrm{E}_{<n}\right)$ merges worlds that so far have not been determined to be distinct and whose labels contain a given formula, if their number exceeds $n-1$.

The application of all rules in Fig. 31 is restricted to the nodes that the indicated form of blocking was not previously applied to. As mentioned above, even if a node was at some stage of derivation subjected

8 Unrestricted blocking does not jeopardize the completeness of such logic, since it subsumes all blocking mechanisms, including pairwise blocking. 


\section{Expansion rules:}

$(\wedge)$ - rule: if $\quad \psi_{1} \wedge \psi_{2} \in \mathcal{L}(v), v$ is not indirectly blocked, and $\left\{\psi_{1}, \psi_{2}\right\} \nsubseteq \mathcal{L}(v)$, then $\mathcal{L}(v) \longrightarrow \mathcal{L}(v) \cup\left\{\psi_{1}, \psi_{2}\right\}$.

$(\vee)$ - rule: if $\psi_{1} \vee \psi_{2} \in \mathcal{L}(v), \quad v \quad$ is not indirectly blocked, and $\left\{\psi_{1}, \psi_{2}\right\} \cap \mathcal{L}(v)=\varnothing$,

then $\quad \mathcal{L}(v) \longrightarrow \mathcal{L}(v) \cup\{\chi\}$ for some $\chi \in\left\{\psi_{1}, \psi_{2}\right\}$.

$\left(\nabla_{i}\right)$ - rule: if $\nabla_{i} \psi \in \mathcal{L}(v), v$ is not blocked, and $v$ has no $R_{i}$-successor $w$ of $v$ such that $\psi \in \mathcal{L}(w)$,

then create a new node $w$ such that $\mathcal{L}(\langle v, w\rangle)=\left\{R_{i}\right\}$, and $\mathcal{L}(w)=\{\psi\}$.

$\left(\square_{i}^{1}\right)$ - rule: if $\square_{i} \psi \in \mathcal{L}(v), v$ is not indirectly blocked, and $\square_{\mathcal{A}_{i}} \psi \notin \mathcal{L}(v)$,

then $\quad \mathcal{L}(v) \longrightarrow \mathcal{L}(v) \cup\left\{\square \square_{\mathcal{A}_{i}} \psi\right\}$.

$\left(\square_{i}^{2}\right)$ - rule: if $\square_{\mathcal{A}_{i}(p)} \psi \in \mathcal{L}(v), v$ is not indirectly blocked, $p \stackrel{R_{i}}{\longrightarrow} q \in \mathcal{A}_{i}$, and there is an $R_{i}$-successor $w$ of $v$ such that $\square_{\mathcal{A}_{i}(q)} \psi \notin \mathcal{L}(w)$,

then $\mathcal{L}(w) \longrightarrow \mathcal{L}(w) \cup\left\{\square_{\mathcal{A}_{i}(q)} \psi\right\}$.

$\left(\square_{i}^{3}\right)$ - rule: if $\square_{\mathcal{A}_{i}} \psi \in \mathcal{L}(v), \quad v$ is not indirectly blocked, $\epsilon \in L\left(\mathcal{A}_{i}\right)$, and $\psi \notin \mathcal{L}(v)$,

then $\quad \mathcal{L}(v) \longrightarrow \mathcal{L}(v) \cup\{\psi\}$.

(ch) - rule: if $\mathrm{E}_{<n} \psi \in \mathcal{L}(v), v$ is not indirectly blocked, and there is an $R_{i}$ successor $w$ of $v$ such that $\{\psi, \dot{\neg} \psi\} \cap \mathcal{L}(w)=\varnothing$,

then $\mathcal{L}(v) \longrightarrow \mathcal{L}(v) \cup\{\chi\}$ for some $\chi \in\{\psi, \dot{\neg} \psi\}$.

$\left(E_{>n}\right)$-rule: if $E_{>n} \psi \in \mathcal{L}(v), \quad v \quad$ is not blocked, and there are no $w_{1}, \ldots, w_{n+1} \in V$ such that $\psi \in \mathcal{L}\left(w_{i}\right)$ and $w_{i} \neq w_{j}$ for $1 \leq i \neq j \leq n+1$

then create $n+1$ new nodes $w_{1}, \ldots, w_{n+1}$ such that $\mathcal{L}\left(w_{i}\right)=\{\psi\}$, and $w_{i} \neq w_{j}$ for $1 \leq i \neq j \leq n+1$.

$\left(\mathrm{E}_{<n}\right)$-rule: if $\mathrm{E}_{<n} \psi \in \mathcal{L}(v), \quad v \quad$ is not indirectly blocked, $\operatorname{Card}(\{w \in V \mid \psi \in \mathcal{L}(w)\}) \geq n, \quad$ and for some $w, z \in\{w \in W \mid \psi \in \mathcal{L}(w)\}$ such that not $w \neq z$, and $w$ is not an ancestor of $z$,

then $\quad$ 1. $\mathcal{L}(z) \longrightarrow \mathcal{L}(z) \cup \mathcal{L}(w)$,

2. if $z$ is an ancestor of $v$, then $\mathcal{L}(\langle z, v\rangle) \longrightarrow \mathcal{L}(\langle z, v\rangle) \cup \operatorname{Inv}(\mathcal{L}(\langle v, w\rangle))$, else $\mathcal{L}(\langle v, z\rangle) \longrightarrow \mathcal{L}(\langle v, z\rangle) \cup \mathcal{L}(\langle v, w\rangle)$,

3. remove $w$ and the sub-tree below $w$.

Figure 31: Rules of the constructive tableau calculus for the logic $\mathcal{M}\left(E_{n}\right)$, based on the calculus for the logic $\mathcal{S H \mathcal { I }}$. 
to blocking, it can be unblocked if it does not satisfy the blocking conditions any more, e.g. as a result of merging another node into it that changed its label.

Remark 8.1. Usually, if no nominals in an explicit form are involved in a calculus, a completion graph resulting from a derivation is expected to be connected and forming a tree. In our version, however, the connectedness of a graph is lost - we obtain a forest rather than a tree. The reason for that is that we do not treat the $E_{>}, E_{<n}$ operators introducing explicit accessibility edges to a graph, even though it is implicitly associated with a total relation over $G$. Nodes introduced by $\left(E_{>n}\right)$ applied to a node $v$ are initially link-free, and might remain so till the end of a derivation. Introducing an explicit accessibility relation (or any approximation of such a relation, for details see [51]) for $\mathbf{E}_{>n}$ to the calculus would require adding some rules that would handle its totality, i.e. reflexivity, transitivity, symmetry and subsumption of all other accessibility relations. It was proven, however, that if we reason about logics with counting operators attached to non-simple accessibility relations, we lose decidability [48]. An accessibility relation $R$ is called simple, if it does not appear on the right hand-side of any of accessibility relation inclusions, unless for all $R_{i}$ such that $R_{i} \subseteq R, R_{i}$ is a simple accessibility relation. An inverse relation $R^{-}$is simple whenever $R$ is simple. Due to the transitivity of $R_{\mathrm{E}}$ (the accessibility relation attached to the $\mathrm{E}$ operator, i.e. the universal relation), the inclusion $R_{\mathrm{E}} \circ R_{\mathrm{E}} \subseteq R_{\mathrm{E}}$ is involved in making $R_{\mathrm{E}}$ non-simple. Thus, to preserve decidability we need to give up the connectedness of the graph.

$\left(\diamond_{i}\right)$ and $\left(E_{>n}\right)$ are generating rules, as they are introducing new nodes to a graph. $\left(E_{<n}\right)$ is the only shrinking rule which can remove nodes from a graph.

Point 2. of $\left(E_{<n}\right)$ describes merging of one node into another one. Intuitively, if it does not lead to a clash, one node can "inherit" a label and some out- and ingoing accessibility edges from another node. If a node was merged into another one, it is removed together with all its descendants. This process is called pruning and is captured by point 3. of $\left(\mathrm{E}_{<n}\right)$. 
The shrinking rule has the highest priority in application, which means that we apply it at the earliest convenience. The remaining rules are applied in arbitrary order.

THEOREM 8.15 ( [46]). The constructive tableau algorithm for the logic $\mathcal{M}\left(\mathrm{E}_{n}\right)$ terminates.

Following [46], we can estimate the maximal size of a graph for $m \cdot 2^{n_{\max }} \cdot 2^{2 m n}$, where $m=\operatorname{Card}(\operatorname{Fclos}(\varphi)), n_{\max }=\max \left\{n \mid \mathrm{E}_{>n} \in\right.$ Fclos $(\varphi)\}, n_{\max }$ coded in binary, and $n$ is a number of accessibility relations occurring in $\varphi$ and a set of accessibility relation inclusions. Such a bound follows from the fact that there are at most $2^{2 m n}$ different labellings of a pair of nodes and an edge between them, so when a length of a path in a graph exceeds $2^{2 m n}$, it immediately gets subjected to pairwise blocking. In every node of such a path, the rule $\left(E_{>_{n}}\right)$ can generate at most $2^{n_{\max }}$ nodes for each $\psi \in \operatorname{Fclos}(\varphi)$, and each accessibility relation, which means that we can have at most $n \cdot m \cdot 2^{n_{\max }}$ such formulas. This gives a NEXPTIME class for running of the algorithm. However, if we take into account the set of inclusions of accessibility relations and the construction of NFA attached to each relation occurring in this set, we obtain the 2NEXPTIME bound for the algorithm, which makes it practically ineffective. Of course, if we only involve one accessibility relation and express frame conditions by explicit rules (Fig. 32), we remain in NEXPTIME.

THEOREM 8.16 (Completeness [47]). The constructive tableau algorithm for the logic $\mathcal{M}\left(\mathrm{E}_{n}\right)$ yields a complete and clash-free completion graph for $\varphi$ as an input if, and only if, there exists a tableau for $\varphi$ that satisfies (P0)-(P10).

We boldly describe how we obtain a tableau out of a complete, clash-free completion graph $G$. We call all nodes in $G$, save an initial node $v$, blockable. Let $b(v)$ denote a node that blocks $v$. A path $p$ is a sequence of pairs of nodes: $\left\langle v_{1}, v_{1}^{\prime}\right\rangle, \ldots\left\langle v_{n}, v_{n}^{\prime}\right\rangle$. We set Tail $(p)=v_{n}$ and Tail' $(p)=v_{n}^{\prime}$. Let $\left\langle p \mid\left\langle v_{n+1}, v_{n+1}^{\prime}\right\rangle\right\rangle=\left\langle v_{1}, v_{1}^{\prime}\right\rangle, \ldots,\left\langle v_{n+1}, v_{n+1}^{\prime}\right\rangle$. We inductively define a path as follows:

1. for each root node $v$ (a node not having any predecessors), $\langle v, v\rangle \in \operatorname{Paths}(\mathrm{G})$, 
Frame conditions rules:

$$
\begin{aligned}
& \text { (ref) - rule: if } \operatorname{Ref}(R), v \text { is not blocked, and } R \notin \mathcal{L}(\langle v, v\rangle) \text {, } \\
& \text { then add an edge }\langle v, v\rangle \text { if it is not yet a part of a graph, and set }
\end{aligned}
$$

Figure 32: Explicit rules for frame conditions.

2. if $p \in \operatorname{Paths}(\mathbf{G}), w$ is a successor of Tail $(p)$ and $w$ is not blocked, then $\langle p \mid\langle w, w\rangle\rangle \in \operatorname{Paths}(\mathbf{G})$,

3. if $p \in \operatorname{Paths}(\mathbf{G}), w$ is a successor of Tail $(p)$ and $w$ is blocked, then $\langle p \mid\langle b(w), w\rangle\rangle \in \operatorname{Paths}(\mathbf{G})$.

Note that a blocked node is always a successor of a node that it is blocked by. This means that whenever a path ends with a pair $\langle v, b(v)\rangle$, we can infinitely extend it by consecutively adding replicated copies of $\langle b(v), v\rangle$. Since in such a path $p \operatorname{Tail}(p)=b(v)$, a pair $\langle b(v), v\rangle$ will indefinitely satisfy the condition 3 . We can define a tableau $T=$ $\left\langle\mathrm{S}, \mathcal{L}^{\prime}, \mathcal{E}\right\rangle$ in the following way:

$$
\mathrm{S}=\operatorname{Paths}(\mathrm{G}) \text {, }
$$

$\mathcal{L}^{\prime}(p)= \begin{cases}\text { Tail }(p) & \text { if } v \text { is a blockable node, } \\ \mathcal{L}(v) & \text { if } v \text { is a root node, }\end{cases}$

$\mathcal{E}(R)=\left\{\langle v, w\rangle \in \mathrm{S} \times \mathrm{S} \mid v=\left\langle p \mid\left\langle z, z^{\prime}\right\rangle\right\rangle, z^{\prime}-R\right.$-successor of Tail $(w)$, or $w=\left\langle q \mid\left\langle z, z^{\prime}\right\rangle\right\rangle$ and $z^{\prime}$ is an $R^{-}$-successor of Tail $\left.(v)\right\} \cup$ $\{\langle v, w\rangle \in \mathrm{S} \times \mathrm{S} \mid v, w$-root nodes, $w$-R-successor of $v\}$. 
The rest of this direction of the proof requires a rather tedious verification whether (P0)-(P10) are satisfied. For details see [51]. For the other direction, we need to suitably apply the non-deterministic rules $(\mathrm{ch})$ and $\left(\mathrm{E}_{>n}\right)$ to obtain a clash-free completion graph that will reflect our tableau.

Observe that a construction of a tableau $T$ out of a completion graph $G$ yields an infinite tableau whenever there are some blocked nodes in the graph, by "unravelling" pairs containing such a node into an infinite path. This means that, consequently, we will obtain a description of an infinite model even in relatively uncomplicated cases like the one presented in Fig. 29, where a blocking mechanism consisting in a simple loop-checking or pattern-checking would suffice to return a significantly reduced (in the case of unrestricted blocking even minimal), finite model. That could lead to a conclusion that pairwise blocking is too coarse and, in a sense, ineffective in comparison to other blocking mechanisms. This, however, would be too hasty. Pairwise blocking was designed to deal with logics that are more expressive than $\mathcal{M}\left(\mathrm{E}_{n}\right)$ and in which specific interferences between nominals, inverse modalities and counting subscripts in modal operators lead to a loss of the finite model property. In such logics, like in the description logic $\mathcal{S H O I \mathcal { ~ }}$ pairwise blocking is the only blocking mechanism that does not break the completeness of a whole calculus.

\subsubsection{Constructive tableau calculus with arithmetic reasoning}

In the previous section, we described a constructive tableau calculus for the logic $\mathcal{M}\left(E_{n}\right)$. The mechanism responsible for termination of the calculus was the pairwise blocking mechanism. One inconvenience that we pointed out was the size of a model yielded from a completion graph, which was infinite whenever there occurred a blocked node in a complete graph. ${ }^{9}$ The other problem is the way the nondeterministic rules (ch) and $\left(\mathrm{E}_{<n}\right)$ are applied. For let $v \in V$ and $\mathrm{E}_{>3} \varphi \wedge \mathrm{E}_{>4} \psi \wedge \mathrm{E}_{<5}(\neg \varphi \vee \neg \psi) \in \mathcal{L}(v)$. Then the application of the

9 Note that even if we take under consideration a graph itself, then if it contains blocked nodes, its size will be bigger than the size of a minimal model since the latter contains loops not searched for by pairwise blocking. 
rule $\left(E_{>n}\right)$ blindly introduces 7 new nodes to a graph. After that the rule (ch) non-deterministically assigns to each node (including the initial node) either $\neg \varphi \vee \neg \psi$ or $\varphi \wedge \psi$. Note that there are $2^{8}$ possible assignments. If either of the assignments results in the excess of 5 nodes in which $\neg \varphi \vee \neg \psi$ holds, then the rule $\left(\mathrm{E}_{<n}\right)$ non-deterministically merges two worlds. Each application of this rule to our formula yields $\left(\begin{array}{l}5 \\ 2\end{array}\right)=10$ possible mergings, so, in total, we have $10^{n-4}$ possible consecutive mergings, where $n=\operatorname{Card}(\{v \in V \mid \neg \varphi \vee \neg \psi \in \mathcal{L}(v)\})$.

The calculus presented in this section attempts to externalize the algebraic part of the reasoning, thus optimising the performance of the non-deterministic rules' application and, at the same time, reducing the size of a graph. Primarily, the calculus was designed for the description logic $\mathcal{A L C Q}$ in [31] and further extended on the logic $\mathcal{S H O Q}$ in [30]. However, the logic $\mathcal{M}\left(E_{n}\right)$ involves global counting operators which $\mathcal{A L C Q}$ lacks. This makes the calculus presented below significantly differ from the original version and, to our best knowledge, novel in the literature. It requires conducting independent soundness, completeness and termination proofs, which is done in the latter part of the section.

The algebraic part of the reasoning requires introducing the notion of atomic decomposition ([72]). In the forthcoming part of the section we briefly explain this technique. Consider a multi-modal logic with three accessibility relations $R_{i}, i=1,2,3$, and graded modalities $\nabla_{>n}^{i}$, $\nabla_{<n}^{i}$ assigned to each relation. Suppose that $\mathfrak{M}=\left\langle W,\left\{R_{i}\right\}_{i=1}^{3}, V\right\}$ is a model for this logic and $w \in W$. By $R_{i}$-fillers for $w$ we denote a set $\left\{v \in W \mid\langle w, v\rangle \in R_{i}\right\}$. Henceforth, we abbreviate a set of $R_{i}$-fillers for $w$ as $R_{i}(w)$. Observe that $R_{i}(w)$ and $R_{j}(w), i \neq j$ do not have to be necessarily disjoint, so it might be the case that $R_{i}(w) \cap R_{j}(w) \neq \varnothing$.

Regarding [72], we suitably formulate a formal definition of the atomic decomposition:

DEFINITION 8.8. Let $\mathfrak{M}=\left\langle W,\left\{R_{i}\right\}_{i=1}^{n}, V\right\rangle$ be a model of a logic, $w \in W, R_{i_{1}}, \ldots, R_{i_{m}}$ be accessibility relations such that $\exists v(\langle w, v\rangle \in$ $\left.R_{i_{m}}\right), i=1, \ldots, m$, and $\mathcal{P}=\mathcal{P}\left(\left\{R_{i_{m}}\right\}_{i=1}^{m}\right)$ Then by atomic decomposition of the set of all $R_{i}$-fillers for $w$ we denote the set:

$$
\left\{\bigcap_{R_{i} \in S} R_{i}(w) \backslash \bigcup_{R_{j} \notin S} R_{j}(w) \mid S \in \mathcal{P}\right\} .
$$


Intuitively, atomic decomposition is a partition of the set of all fillers for $w$ into disjoint sets of fillers with respect to all accessibility relations from certain subset of $R_{i_{1}}, \ldots, R_{i_{m}}$, and only them. The picture below exhibits all possible mutual relations between $R_{i}(w), i=1,2,3$.

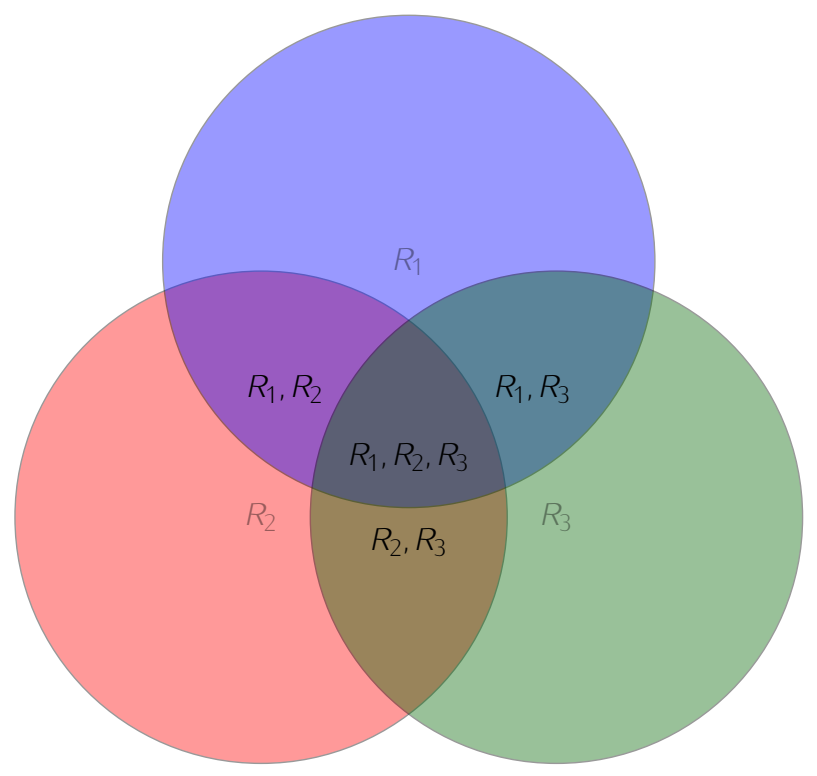

To make use of the whole atomic decomposition toolkit, we need to introduce a special rewriting function Tr for formulas involving cardinality constraints. We do it as follows. Assume that $\mathrm{E}_{>n} \varphi, \mathrm{E}_{<n} \varphi \in \mathcal{L}(v)$ for some $v \in V$. Then

$$
\begin{aligned}
& \operatorname{Tr}\left(\mathrm{E}_{>n} \varphi\right)=\diamond_{>n}^{(\varphi, v,>n)} \varphi \wedge \square^{(\varphi, v,>n)} \varphi \\
& \operatorname{Tr}\left(\mathrm{E}_{<n} \varphi\right)=\diamond_{<n}^{(\varphi, v,<n)} \varphi \wedge \square^{(\varphi, v,<n)} \varphi \wedge \square^{(\varphi, \tilde{v},<n)} \neg \varphi,
\end{aligned}
$$

where $\nabla_{>n}^{(\varphi, v,>n)}$ and $\nabla_{<n}^{(\varphi, v,<n)}$ are attached to freshly introduced accessibility relations $R^{(\varphi, v,>n)}, R^{(\varphi, v,<n)}$ which are not assumed to be of any particular kind. A rewriting of a formula $\mathrm{E}_{<n} \varphi$ also introduces the additional accessibility relation $R^{(\varphi, v,<n)}$ which is a complement of $R^{(\varphi, v,<n)}\left(R_{\mathrm{E}} \backslash R^{(\varphi, v,<n)}\right)$. Thus, every occurrence of a formula with cardinality restriction in a graph introduces an auxiliary accessibility relation $R^{(\varphi, v,<n)} \subseteq R_{\mathrm{E}}$ or $R^{(\varphi, v,>n)} \subseteq R_{\mathrm{E}}$, and, in the case of $\mathrm{E}_{<n^{-}}$ formulas, also $R^{(\underline{\varphi, v},>n)} \subseteq R_{\mathrm{E}}$. We only know that each introduced 
accessibility relation is subsumed by $R_{\mathrm{E}}$ but we do not know anything about mutual subsumptions between them. Note that since we have not assumed anything about these freshly introduced accessibility relations (e.g. that they are transitive or reflexive), we do not need to be concerned that reasoning with counting formulas will turn out undecidable as violating the conditions of simplicity of accessibility relations (for the exact definition of simple accessibility relation see Section 8.6.2 of this chapter).

Like in the previous section, we dispense with the explicit handling of $R_{\mathrm{E}}$ as an accessibility relation, due to its universal range. However, all auxiliary relations will be handled in a similar way to the $\diamond$-accessibility relation.

The last remark we need to make before we proceed to a formal definition of a tableau is that for the sake of completeness of the whole arithmetic reasoning and, what follows, the calculus, we will treat $\diamond$ as a graded modality. We therefore extend the function $\mathrm{Tr}$ in the following manner:

$$
\operatorname{Tr}(\diamond \varphi)=\diamond_{>0}^{(v, \varphi, \diamond)} \varphi \wedge \square^{(v, \varphi, \diamond)} \varphi,
$$

where $R^{(v, \varphi, \diamond)} \subseteq R$. As will turn out soon, there is no necessity for rewriting $\square$ formulas ${ }^{10}$.

Now we are ready to define a tableau (cf. Definition 8.6). We assume that models for our logic are defined over the signature $\langle\mathrm{PROP}, R\rangle$.

Definition 8.9. $\mathrm{T}=\langle\mathrm{S}, \mathcal{L}, \mathcal{E}\rangle$ is a tableau for a formula $\varphi$ if, and only if:

$\mathrm{S}$ is a non-empty set,

$\mathcal{L}: \mathrm{S} \longrightarrow 2^{\operatorname{clos}(\varphi)}$ maps each element to a set of formulas,

$\mathcal{E}: \mathrm{R} \longrightarrow 2^{\mathrm{S} \times \mathrm{S}}$ maps each accessibility relation to a set of pairs of elements in $\mathrm{S}$.

Additionally, for all $s, t \in \mathrm{S}, \psi, \psi_{1}, \psi_{2} \in \operatorname{clos}(\varphi)$ and $R_{i} \in \mathrm{R}, T$ satisfies the following conditions:

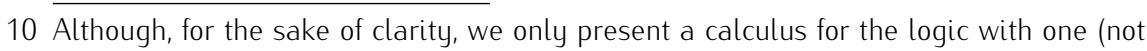
auxiliary) accessibility relation, it is clear from the construction of the calculus that it can be easily extended to the multi-modal case. 
(P0) there is some $s \in \mathrm{S}$ with $\varphi \in \mathcal{L}(s)$,

(P1) if $\psi \in \mathcal{L}(s)$, then $\dot{\neg} \psi \notin \mathcal{L}(s)$,

(P2) if $\psi_{1} \wedge \psi_{2} \in \mathcal{L}(s)$, then $\psi_{1} \in \mathcal{L}(s)$ and $\psi_{2} \in \mathcal{L}(s)$,

(P3) if $\psi_{1} \vee \psi_{2} \in \mathcal{L}(s)$, then $\psi_{1} \in \mathcal{L}(s)$ or $\psi_{2} \in \mathcal{L}(s)$,

(P4) if $\square^{i} \psi \in \mathcal{L}(s)$ and $\langle s, t\rangle \in \mathcal{E}\left(R^{i}\right)$, then $\psi \in \mathcal{L}(t)$,

(P5) if $\square^{\tilde{i}} \psi \in \mathcal{L}(s)$ and $\langle s, t\rangle \notin \mathcal{E}\left(R^{i}\right)$, then $\psi \in \mathcal{L}(t)$,

(P6) if $\diamond_{>n}^{i} \psi \in \mathcal{L}(s)$, then $\operatorname{Card}\left(\left\{t \mid\langle s, t\rangle \in \mathcal{E}\left(R^{i}\right)\right.\right.$ and $\psi \in$ $\mathcal{L}(t)\})>n$,

(P7) if $\diamond_{<n}^{i} \psi \in \mathcal{L}(s)$, then $\operatorname{Card}\left(\left\{t \mid\langle s, t\rangle \in \mathcal{E}\left(R^{i}\right)\right.\right.$ and $\psi \in$ $\mathcal{L}(t)\})<n$,

(P8) if $\langle s, t\rangle \in \mathcal{E}\left(R^{(i, \diamond)}\right)$, then $\langle s, t\rangle \in \mathcal{E}(R)^{11}$.

THEOREM 8.17 (Tableau completeness). A $\mathcal{M}\left(\mathrm{E}_{n}\right)$ formula $\varphi$ is satisfiable if, and only if there exists a tableau for $\varphi$, satisfying (P0)-(P8).

Proof. We proceed in two directions.

$(\Leftarrow)$ Suppose that $\mathrm{T}=\langle\mathrm{S}, \mathcal{L}, \mathcal{E}\rangle$ is a tableau for a formula $\varphi$. Let $\mathfrak{M}=\langle W, R, V\rangle$, where:

$W:=\mathrm{S}$,

$R:=R \in \mathrm{R}$,

$V(P)=\{w \in W \mid p \in \mathcal{L}(w)\}$, for each $p \in \operatorname{PROP}(\varphi)$.

We now prove that $\mathfrak{M}$ is a model for $\varphi$. We proceed by induction on $\psi \in \operatorname{Sub}(\varphi)$. Basic case and Boolean cases are trivial and follow immediately from (P0)-(P3) of Definition 8.9 and the definition of $V$.

$\psi=\diamond \chi$ Suppose that $\nabla_{x} \in \mathcal{L}(v)$ for some $v \in \mathrm{S}$. After rewriting chi turned into $\diamond_{>0}^{(v, x, \diamond)} \chi \wedge \square^{(v, x, \diamond)} \chi$. By (P6) it follows that there exists at least $1 w \in \mathrm{S}$ such that $\langle v, w\rangle \in \mathcal{E}\left(R^{(v, x, \diamond)}\right)$ and $\chi \in \mathcal{L}(w)$. Since by (P8) $\langle s, t\rangle \in \mathcal{E}(R)$, then by the inductive hypothesis we obtain $\mathfrak{M}, v \models \chi$.

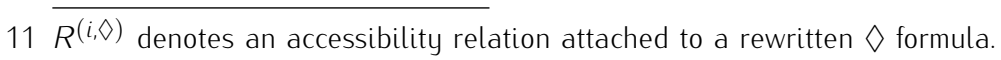


$\psi=\square \chi$ Suppose that $\square x \in \mathcal{L}(v)$ for some $v \in \mathrm{S}$. This formula was not subjected to rewriting. Then by (P4) we have that for all $v \in \mathrm{S}$, such that $\langle w, v\rangle \in \mathcal{E}(R), \chi \in \mathcal{L}(w)$. By the definition of $R$ in $\mathfrak{M}$ and by the inductive hypothesis we obtain $\mathfrak{M}, v \models \square$.

$\psi=\mathrm{E}_{>n} X$ Suppose that $\mathrm{E}_{>n} X \in \mathcal{L}(v)$ for some $v \in \mathrm{S}$. Applying $\operatorname{Tr}$ to this formula yields $\diamond_{>n}^{(x, v,>n)} \chi \wedge \square^{(x, v,>n)} \chi$. By (P6) we know that $\operatorname{Card}\left(\left\{w \mid\langle v, w\rangle \in \mathcal{E}\left(R^{i}\right)\right.\right.$ and $\left.\left.\chi \in \mathcal{L}(w)\right\}\right)>n$, and, what follows, $\operatorname{Card}(\{w \mid \chi \in \mathcal{L}(w)\})>n$. By the inductive hypothesis we conclude that $\mathfrak{M}, v \mid=\mathrm{E}_{>n X}$.

$\psi=\mathrm{E}_{<n} X$ Suppose that $\mathrm{E}_{<n} X \in \mathcal{L}(v)$ for some $v \in \mathrm{S}$. This formula was rewritten to $\diamond \stackrel{(x, v,<n)}{<n}^{(x} \wedge \square^{(x, v,<n)} \chi \wedge \square^{\left(x, v_{i}<n\right)} \chi$. Let $M=\left\{w \mid\langle v, w\rangle \in \mathcal{E}\left(R^{i}\right)\right.$ and $\left.\chi \in \mathcal{L}(w)\right\}$. By (P6) we know that $\operatorname{Card}(M)<n$. Additionally, by (P5) and (P1) we infer that $\operatorname{Card}(\{w \in S \backslash M \mid X \in \mathcal{L}(w)\})=0$. We can therefore conclude that $\operatorname{Card}(\{w \mid x \in \mathcal{L}(w)\})<n$. By the inductive hypothesis we obtain $\mathfrak{M}, v \mid=\mathrm{E}_{<n} X$.

$(\Leftarrow)$ Suppose that $\mathfrak{M}=\langle W, R, V\rangle$ is a model for $\varphi$. Then we construct a tableau $\mathrm{T}=\langle\mathrm{S}, \mathcal{L}, \mathcal{E}\rangle$ in the following manner. Let $\mathrm{S}=W$ and $\mathcal{L}(v)=\{\psi \in \operatorname{clos}(\varphi)|\mathfrak{M}, v|=\psi\}$. For $\mathcal{E}$ we do the following. For each $v \in \mathrm{S}$ such that $\mathrm{E}_{>n} \psi \in \mathcal{L}(v)$ we search for nodes $w_{1}, \ldots, w_{n+1}$ such that $\psi \in \mathcal{L}\left(w_{i}\right), i=1, \ldots n+1$. By the definition of $\mathbf{S}$ and $\mathcal{L}$, and the fact that $\mathfrak{M}$ is a model for $\varphi$, we are certain that such worlds exist. Then we set $\mathcal{E}\left(R^{(v, \psi,>n)}\right)=\left\{\left\langle v, w_{i}\right\rangle \mid\right.$ $i=1, \ldots n+1\}$. Next, for each $v \in \mathrm{S}$ such that $\mathrm{E}_{<n} \psi \in \mathcal{L}(v)$ we search for all nodes $w$ such that $\mathbf{E}_{>n} \psi \in \mathcal{L}(w)$. By the fact that $\mathfrak{M}$ is a model for $\varphi$, we know that there is at most $n-1$ such worlds. We set $\mathcal{E}\left(R^{(v, \psi,<n)}\right)=\{\langle v, w\rangle \mid \psi \in \mathcal{L}(w)\}$. Subsequently, for each $v \in \mathrm{S}$ such that $\nabla \psi \in \mathcal{L}(v)$ we seek for all nodes $w$ such that $\langle v, w\rangle \in R$ in $\mathfrak{M}$ and $\psi \in \mathcal{L}(w)$. We set $\mathcal{E}\left(R^{(v, \psi, \diamond)}\right)=\{w \in \mathrm{S} \mid\langle v, w\rangle \in R$ in $\mathfrak{M}$ and $\psi \in \mathcal{L}(w)\}$. Finally, we set $\mathcal{E}(R)=\{\langle v, w\rangle \mid\langle v, w\rangle \in R$ in $\mathfrak{M}\}$.

We need to verify that $\mathrm{T}$ satisfies (P0)-(P8). (P0)-(P3) straightforwardly follow from the definition of $\mathcal{L}$ and $S$, and the fact that $\mathfrak{M}$ is a model for $\varphi$. Satisfaction of (P4) is a consequence of the definition of $\mathcal{E}$ for 
$R^{(v, \psi,>n)}, R^{(v, \psi, \diamond)}$ and $R$ (all types of accessibility relations that can be attached to the $\square^{i}$ operator from (P4)). For (P5) observe that $\square^{\tilde{i}}$ operators only occur in rewritten $\mathrm{E}_{<n}$ formulas. By the definition of $\mathcal{E}$, the accessibility relations of the form $R^{(v, \psi,<n)}$ link $v$ with all worlds $w$ such that $\psi \in \mathcal{L}(w)$, and by the fact that $\mathfrak{M}$ is a model for $\varphi$, we conclude that (P5) holds. For (P6) note that formulas of the form $\nabla_{<n}^{i} \psi$ can only occur after rewriting of $\mathrm{E}_{>n}$ or $\diamond$ formulas. In the first case, (P6) easily follows from the definition of $\mathcal{E}$ for $R^{(v, \psi,>n)}$. In the latter case, it follows from the definition of $\mathcal{E}$ for $R^{(v, \psi, \diamond)}$ and the fact that $\mathfrak{M}$ is a model for $\varphi$. (P7) is a result of the definition of $\mathcal{E}$ for $R^{(v, \psi,<n)}$. (P8) is implied by the definition of $\mathcal{E}$ for $R$ and $R^{(v, \psi, \diamond)}$.

This concludes the proof.

The tableau algorithm which we present in the remainder of the section needs a suitably tailored notion of a completion graph. We provide it in the following definition:

Definition 8.10 (Completion graph). Let $\varphi$ is a $\mathcal{M}\left(\mathrm{E}_{n}\right)$-formula. A completion graph for $\varphi$ is a directed graph $G=\langle V, E, \mathcal{L}, E q\rangle$, such that:

1. each node $v \in V$ is labelled with a set $\mathcal{L}(v) \subseteq \operatorname{clos}(\varphi)$,

2. each edge $\langle v, w\rangle \in E$ is labelled with a set $\mathcal{L}(\langle v, w\rangle) \subseteq \mathrm{R}$, where $\mathrm{R}$ - the set of accessibility relations occurring in a graph during the derivation,

3. Eq is a set of tuples of the form $\xi=\left\langle v, \gtrless n, R^{i}\right\rangle$, and inequalities of the form $\mathrm{p}_{S}^{c} \gtrless n$, where $v \in V, \psi \in \operatorname{clos}(\varphi), \gtrless \in\{>,<\}, R^{i}$ is of the form $R^{(v, \psi, \gtrless n)}$ or $R^{(v, \psi, \diamond)}$, and $\mathrm{p}_{S}$ is a partition (for an explanation of the notion of a partition see a paragraph devoted to the inequality solver below.)

We say that $G$ contains a clash if one of the following conditions is satisfied:

1. $\perp \in \mathcal{L}(v)$ for some $v \in V$,

2. $\{\psi, \neg \psi\} \subseteq \mathcal{L}(v)$ for some $v \in V$ and $\psi \in \operatorname{Sub}(\varphi)$, 
3. There exists no non-negative solution of a set of inequalities Eq.

A graph $\mathrm{G}$ is called complete if no further rules are applicable to any of its nodes.

Before we proceed to a detailed description of how our algorithm performs, a word of explanation needs to be devoted to the set Eq and the inequality solver employed to cope with the arithmetical reasoning within our algorithm. Suppose that $\xi_{1}, \ldots, \xi_{n} \in$ Eq. Each $\xi_{i}$ is correlated with some $R^{i}$. Note that $R^{i} \neq R_{j}$ whenever $i \neq j$. To each $R^{i}$ there is attached a name of a node from $V$, let's denote it by $v^{i}$. First, the inequality solver counts the power set of the set of all names of accessibility relations occurring in Eq, enriched with $R^{\varnothing}$ which denotes the empty relation (intuitively, it covers the case of all nodes not being fillers of any accessibility relation), we call it $\mathcal{P}$. Now, it takes a function $\alpha$ which assigns to each $S \in \mathcal{P}$ a set $\mathrm{p}_{S}=\left\{\bigcap_{R^{i} \in S} R^{i}\left(v^{i}\right) \backslash \bigcup_{R^{j} \notin S} R^{j}\left(v^{j}\right)\right\}$. Obviously, $\mathrm{p}_{S_{i}} \cap \mathrm{p}_{S_{j}}=\varnothing$ for $S_{i} \neq S_{j}$. Pictorially, each non-empty set $S$ containing names of accessibility relations is mapped to the set $p_{S}$ of all nodes that are $R^{i}$-fillers for all and only $R^{i} \in S$. All sets $\mathrm{p}_{S_{i}}$ are mutually disjoint. Each $\xi_{1} \in \mathrm{Eq}$ imposes some cardinality constraints on all $\mathrm{p}_{S}$ such that $R^{i} \in S$. For instance, if $\mathrm{Eq}=\left\{\xi_{1}, \xi_{2}\right\}$, where $\left.\xi_{1}=\left\langle v_{1}, \psi_{1},\right\rangle 4, R^{1}\right\rangle$ and $\xi_{2}=\left\langle v_{2}, \psi_{2},<3, R^{2}\right\rangle$, then we know that $\operatorname{Card}\left(\mathrm{p}_{R^{1}} \cup \mathrm{p}_{R^{1}, R^{2}}\right)>4$. Let $\beta$ be a function mapping partitions $\mathrm{p}_{S}$ to their cardinalities denoted here by $\mathrm{p}_{S}^{c}$. Since all distinct partitions are mutually disjoint, $\beta$ is additive and we obtain $\mathrm{p}_{R^{1}}^{c}+\mathrm{p}_{R^{1}, R^{2}}^{c}>4$. Ultimately, if $\mathrm{Eq}=\left\{\xi_{1}, \ldots, \xi_{n}\right\}$, then each $\xi_{i}=\left\langle v_{i}, \gtrless m_{i}, R^{i}\right\rangle$ is turned into an inequality of the form $\mathrm{p}_{S_{1}}^{c}+\cdots+\mathrm{p}_{S_{2^{n-1}}}^{c} \gtrless m_{i}$ such that $R^{i} \in S_{j}$ for $j=1, \ldots, 2^{n-1}$. If a solver returns a solution of the form $\mathrm{p}_{S}^{c}=m$, then we denote $m$ by $\sigma\left(\mathrm{p}_{S}\right)$.

Now we can move to the explication of how the algorithm works. Given a formula $\varphi$ in question, the algorithm starts with a graph: $\left\langle V^{0}, E^{0}, \mathcal{L}^{0}, \mathrm{Eq}^{0}\right\rangle$ where:

$V^{0}=\{v\}$,

$E^{0}=\varnothing$

$\mathcal{L}^{0}(v)=\{\varphi\}$, 


\section{Expansion rules:}

$(\wedge)$ - rule: if $\quad \psi_{1} \wedge \psi_{2} \in \mathcal{L}(v)$, and $\left\{\psi_{1}, \psi_{2}\right\} \nsubseteq \mathcal{L}(v)$, then $\mathcal{L}(v) \longrightarrow \mathcal{L}(v) \cup\left\{\psi_{1}, \psi_{2}\right\}$.

$(\vee)$ - rule: if $\quad \psi_{1} \vee \psi_{2} \in \mathcal{L}(v)$, and $\left\{\psi_{1}, \psi_{2}\right\} \cap \mathcal{L}(v)=\varnothing$, then $\mathcal{L}(v) \longrightarrow \mathcal{L}(v) \cup\{\chi\}$ for some $\chi \in\left\{\psi_{1}, \psi_{2}\right\}$.

$\left(\square_{i}\right)$ - rule: if $\square^{i} \psi \in \mathcal{L}(v)$, there exists $w$ such that $R^{i} \in \mathcal{L}(\langle v, w\rangle)$ or $R^{j} \in$ $\mathcal{L}(\langle v, w\rangle)$ and $R^{j} \subseteq R^{i} \in \operatorname{lncl}$, and $\psi \notin \mathcal{L}(w)$,

then $\quad \mathcal{L}(w) \longrightarrow \mathcal{L}(w) \cup\{\psi\}$.

$\left(\square^{\tilde{i}}\right)$ - rule: if $\square^{\tilde{i}} \psi \in \mathcal{L}(v)$, there exists $w$ such that $R^{i} \notin \mathcal{L}(\langle v, w\rangle)$, and $\psi \notin \mathcal{L}(w)$,

then $\quad \mathcal{L}(w) \longrightarrow \mathcal{L}(w) \cup\{\psi\}$.

(ch)-rule: if there exists $\mathrm{p}_{S}$ occurring in $\mathrm{Eq}$ such that $\left\{\mathrm{p}_{S}>0, \mathrm{p}_{S}<1\right\} \cap \mathrm{Eq}=\varnothing$,

then $\mathrm{Eq} \longrightarrow \mathrm{Eq} \cup\{\eta\}$ for some $\eta \in\left\{\mathrm{p}_{S}>0, \mathrm{p}_{\mathrm{S}}<1\right\}$.

$\left(\diamond_{>n}^{i}\right)$-rule: if $\quad \diamond_{>n}^{i} \psi \in \mathcal{L}(v)$, and $\quad\left\{\left\langle v,>n, R^{(v, \psi,>n)}\right\rangle,\left\langle v,>n, R^{(v, \psi, \diamond)}\right\rangle\right\} \cap$

$\mathrm{Eq}=\varnothing$,

then if $\diamond_{>n}^{i} \psi$ was introduced by rewriting of a $\diamond$ formula,

then $\mathrm{Eq} \longrightarrow \mathrm{Eq} \cup\left\{\left\langle v,>n, R^{(v, \psi, \diamond)}\right\rangle\right\}$

else $\mathrm{Eq} \longrightarrow \mathrm{Eq} \cup\left\{\left\langle v,>n, R^{(v, \psi,>n)}\right\rangle\right\}$.

$\left(\diamond_{<n}^{i}\right)$ - rule: if $\diamond_{<n}^{i} \psi \in \mathcal{L}(v)$, and $\left\langle v,<n, R^{(v, \psi,<n)}\right\rangle \notin$ Eq,

then $\mathrm{Eq} \longrightarrow \mathrm{Eq} \cup\left\{\left\langle v,<n, R^{(v, \psi,>n)}\right\rangle\right\}$.

(fil) - rule: if there exist partitions $p_{S_{1}} \ldots p_{S_{n}}$ occurring in Eq which result from a division of a partition $\mathrm{p}_{S_{0}}$ such that $S_{0} \subseteq S_{i}$ for $i=1, \ldots, n$ and $\sigma\left(\mathbf{p}_{S_{i}}\right)=m_{i}$, and there are already $k \geq 0$ nodes $v_{1}, \ldots, v_{k}$ such that $\left\{R^{l} \mid\right.$ there exists $w \in V$ such that $\left.\left\langle w, v_{j}\right\rangle \in R^{l}\right\}=S_{0}$ for $j=1, \ldots, k$,

then 1. for each $v_{j}, j=1, \ldots, k$ set $\mathcal{L}\left(\left\langle w, v_{j}\right\rangle\right)=R^{r, w}$ for all $R^{r, w} \in S_{i}$ for some $i=1, \ldots, n^{*}$,

2. for each $S_{i}$ for $i=1, \ldots, n$,

if $\operatorname{Card}\left(\left\{v \in V \mid\left\{R^{l} \mid\right.\right.\right.$ there exists $w \in V$ such that $\langle w, v\rangle \in$ $\left.\left.\left.R^{l}\right\}=S_{i}\right\}\right)=c \leq m_{1}$,

then create $c-m_{i}$ new nodes $y_{1}, \ldots, y_{c-m_{i}}$ and for all $l=$ $1, \ldots, c-m_{i}$ set $\mathcal{L}\left(\left\langle w, v_{l}\right\rangle\right)=R^{r, w}$ for each $R^{\gamma, w} \in S_{i}$.

* $R^{\gamma, w}$ is an accessibility relation occurring in Eq and having $w$ in its superscript (it can therefore be of the form $R^{(w, \psi,>n)}, R^{(w, \psi,<n)}$, or $\left.R^{(w, \psi, \diamond)}\right)$.

Figure 33: Rules of the calculus for the logic $\mathcal{M}\left(\mathrm{E}_{n}\right)$, which involves an inequality solver. 


$$
\mathrm{Eq}^{0}=\left\{\mathrm{p}_{\varnothing}^{c}>0\right\}
$$

Directly after initiating the algorithm the only node in $\mathrm{G}$ is the initial node $v$. However, no accessibility relation has occurred yet that would have any fillers in $G$. It means that the only relation appearing in the inequality solver is $R^{\varnothing}$. To divide a graph into partitions, the solver accordingly counts $\mathcal{P}\left(\left\{R^{\varnothing}\right\}\right)$. Since it is a set $\left\{\left\{R^{\varnothing}\right\}, \varnothing\right\}$, the only partition resulting from applying $\alpha$ to $\mathcal{P}(\varnothing)$ is $\mathrm{p}_{R^{\varnothing}}$. Obviously, $v \in \mathrm{p}_{R^{\varnothing}}$ and since all remaining nodes are introduced to a graph as fillers of accessibility relations, the cardinality of $\mathrm{p}_{R^{\varnothing}}$ will never exceed 1 (it might decrease if meanwhile $v$ becomes a filler of some accessibility relation). Hence, $\mathrm{p}_{R^{\varnothing}}>0 \in \mathrm{Eq}$. It is worth stressing the essential difference between an empty partition $\mathrm{p}_{S}$ and a partition defined over the empty accessibility relation $\mathrm{p}_{R^{\varnothing}}$. Whereas the former is necessarily empty and indicates the fact that there are no nodes in $G$ that are simultaneously fillers of all accessibility relations in $S$, the latter might not be empty (as pointed, it can contain at most one node) and might include nodes that are not fillers of any accessibility relation. Note also that for all nodes $v_{1}, \ldots, v_{m}$, save the initial node, within the same partition, name it $\mathrm{P}_{S}, \mathcal{L}\left(v_{i}\right)=\mathcal{L}\left(v_{j}\right), 1 \leq i<j \leq m$. Indeed, due to the way nodes are introduced to a graph, their label is fully determined by a set of accessibility relations they are fillers for. $R^{\varnothing}$ has therefore only auxiliary character and is meant to involve the initial node into the arithmetic reasoning. Whenever a node is ascribed to the partition $\mathrm{p}_{S}$, where $R^{\varnothing} \in S$, it does not mean that it is simultaneously a filler of remaining accessibility relations and a filler of no accessibility relation, but, basically, that it is the initial node.

We apply all rules from Fig. 33, save the (fil)-rule, with equal priority. (fil) has lower priority of application which means that we only apply it when no other rule is applicable. Before we provide a thorough explanation of the tableau rules, two general comments on certain aspects of the algorithm's operation are in place.

First, rewriting is performed "on the fly", once a formula of the form $\mathrm{E}_{>n} \psi, \mathrm{E}_{<n} \psi$ or $\diamond \psi$ occurred in $\mathcal{L}(w)$ for some $w \in V$.

Second, "at side" we keep a set of inclusions of accessibility relations denoted by Incl. At start, Incl $=\varnothing$. Whenever a formula $\nabla_{>0}^{(v, \psi, \diamond)} \psi$ occurs in a graph, the inclusion $R^{(v, \psi, \diamond)} \subseteq R$ is added to Incl. 
The rules $(\wedge)$ and $(\vee)$ act in a standard way. $\left(\square^{i}\right)$ distributes a formula under the scope of $\square^{i}$ to all $R^{i}$ successors of a current node. Moreover, it utilises the set Incl and propagates the formula to all $R^{j}$ successors of a current node whenever $R^{j}$ is a sub-relation of $R^{i}$. The second condition was introduced to appropriately propagate formulas of the form $\square \psi$ which are not rewritten in the process. All accessibility relations introduced by $\diamond_{>0}^{(v, \psi, \diamond)}$ are of auxiliary character and are subsumed by $R$. By means of Incl we can keep track of these subsumptions and avoid them being violated by accordingly using $\left(\square^{i}\right)$. $(\square \tilde{i})$ only applies to formulas resulting from rewriting of $\mathrm{E}_{<n}$. These formulas store information about the upper-bound on the global number of nodes satisfying a formula under the scope of $\mathrm{E}_{<n}$, let's denote it by $\psi$. Therefore, $m$ nodes are picked $(m \leq n)$ in which the formula holds and $\left(\square^{i}\right)$ distributes $\neg \psi$ to all other worlds, so whenever a number of nodes satisfying $\psi$ exceeds $m$, it results in a clash. The (ch) rule is a very interesting instance of a non-deterministic rule. At each stage of derivation the inequality solver divides $V$ into partitions respectively to a set of accessibility relations occurring in Eq. Although certain constraints are imposed on cardinalities of partitions' unions by inequalities in Eq, we have no information about cardinalities of partitions themselves (the inequality solver is supposed to fill this gap). The (ch) rule non-deterministically decides whether a partition should be empty or whether it should contain some elements. One can easily calculate that for $n$ accessibility relations in $\mathrm{Eq}$ we have $2^{2^{n}-1}$ possible choices (there are $2^{n}-1$ partitions and each of them can be decided to be empty or non-empty). The rules $\left(\diamond_{>n}^{i}\right)$ and $\left(\diamond_{<n}^{i}\right)$ operate in a similar way. Instead of introducing new nodes to a graph, they only forward information about the at-least and at-most bounds to the inequality solver. A tuple $\left\langle v,>n, R^{i}\right\rangle$ encapsulates the inequality $\operatorname{Card}\left(R^{i}(v)\right)>n$. Similarly for $\left\langle v,<n, R^{i}\right\rangle$. A whole arithmetic reasoning is delegated to the inequality solver. The (fil) rule looks the most appalling. However, intuitions concealed under this guise are rather simple. Once $\left(\nabla_{>n}^{i}\right),\left(\diamond_{<n}^{i}\right)$ and $(\mathrm{ch})$ were applied, the inequality solver picks up the slack. It solves the set of inequalities present in Eq and yields a full arithmetic description of a graph, i.e. the information about the cardinality of each partition. We can program our solver in such 
a way that it only returns minimal non-negative solutions (where the cardinality of a whole graph is minimal). In fact, we have to do this to assure that all future at-most constraints are met (if a decided formula is satisfiable). The inequality solver is NP-complete (for further discussion on linear integer programming see $[73,75])$, however the number of minimal solutions of the set of inequalities can be in the worst case exponential in the number of partitions. We non-deterministically pick one. According to this solution the (fil) rule is applied. If some new accessibility relation $R^{i}$ was introduced to $\mathrm{Eq}$, the solver repartitions a graph with respect to a new set of accessibility relations. If a set of partitions $\mathrm{p}_{S_{1}}, \ldots, \mathrm{p}_{S_{n}}$ results from dividing an old partition $\mathrm{p}_{S_{S}}$, then (fil) non-deterministically distributes nodes of $p_{S}$ between $p_{S_{1}}, \ldots, p_{S_{n}}$. Afterwards, (fil) complements each $\mathrm{p}_{S_{i}}, i=1, \ldots, n$, by adding new nodes and edges, so that $\mathrm{p}_{S_{i}}^{c}=\sigma\left(\mathrm{p}_{S_{i}}\right), i=1, \ldots, n$. Contrary to $\mathbf{E}_{>n}$ rules in the calculi presented in the last two sections, we can only introduce so many nodes to a partition $\mathrm{p}_{S_{i}}$ that $\mathrm{p}_{S_{i}}^{c}=\sigma\left(\mathrm{p}_{S_{i}}\right)$, and cannot always blindly introduce $\sigma\left(\mathrm{p}_{S_{i}}\right)$ nodes. The reason is that our calculus is not supplied with shrinking rules that could get rid of superfluous nodes at a future stage of derivation.

Since the inequality solver partitions nodes in a graph globally, which means that any node can become a filler of any accessibility relation, it enables us to dispense with blocking. It becomes unnecessary, since whenever a formula is satisfiable, the inequality solver helps us find a finite completion graph for it! What follows, it makes the whole derivation finite. The foregoing, rather loose, remarks are expressed more formally in the following three theorems.

THEOREM 8.18 (Soundness). If the expansion rules from Fig. 33 can be applied to a formula $\varphi$ in such a way that they yield a complete and clash-free graph, then there exists a tableau for $\varphi$.

Proof. The proof is standard (see, e.g., [31, 51]). Assume that $\mathrm{G}=$ $\langle V, E, \mathcal{L}, E q\rangle$ is a graph for $\varphi$. Let $T=\left\langle S, \mathcal{L}^{\prime}, \mathcal{E}\right\rangle$ such that:

$\mathrm{S}=V_{\text {, }}$

$\mathcal{L}^{\prime}(v)=\mathcal{L}(v)$ for each $v \in \mathrm{S}$, 
$\mathcal{E}\left(R^{i}\right)=\left\{\langle v, w\rangle \in E \mid R^{i} \in \mathcal{L}(\langle v, w\rangle)\right\}$ and $\mathcal{E}(R)=\left\{\langle v, w\rangle \in R^{i} \mid\right.$ $\left.R^{i} \subseteq R \in \operatorname{lncl}\right\}$.

It suffices to show that $T$ satisfies conditions (P0)-(P8) from Definition 8.9. (P0) and (P1) hold by the fact that $G$ is a clash-free graph for $\varphi$ and by the definition of T. (P2) and (P3) are satisfied thanks to the rules $(\wedge)$ and $(\vee)$ and the fact that $G$ is complete. $(P 4)$ follows from the applications of $\left(\square^{i}\right)$ in a complete graph and from the definition of $R$ in $\mathrm{T}$. (P5) is a consequence of the fact that $\left(\square^{i}\right)$ was exhaustively applied in G. (P6) and (P7) are obtained as a result of the performance of the inequality solver which, in the face of clash-freeness of $G$, must have yielded a non-negative solution of a set of inequalities introduced by $\diamond_{>n}^{i}$ and $\nabla_{<n}^{i}$ operators. (P8) trivially holds due to the definition of $R$ in $T$.

THEOREM 8.19 (Completeness). Whenever there exists a tableau for a formula $\varphi$, the tableau rules can be applied in such a way that they return a complete and clash-free graph for $\varphi$.

Proof. The proof is conducted along the lines of the one in [31]. Assume that $\mathrm{T}=\left\langle\mathrm{S}, \mathcal{L}^{\prime}, \mathcal{E}\right\rangle$ is a tableau for $\varphi$ which is finite and minimal (in terms of the cardinality of $\mathbf{S}$ ). This assumption is justified since by Theorem $5.16 \mathcal{M}\left(\mathrm{E}_{n}\right)$ enjoys the finite model property and by Theorem 8.17 whenever $\varphi$ has a model, we can construct a tableau of the same size. Our task is to show that having $T$, we can apply the tableau rules in such a way that yields a complete and clash-free graph for $\varphi$. In order to reach that goal, we define inductively with introducing new nodes to a graph a mapping $\pi: V \longrightarrow S$ satisfying the following conditions:

1. for each node $v \in V \mathcal{L}(v) \subseteq \mathcal{L}^{\prime}(\pi(v))$,

2. for each pair of nodes $V, w \in V$ and each accessibility relation $R^{i}$, if $R^{i} \in \mathcal{L}(\langle v, w\rangle)$, then $\langle\pi(v), \pi(w)\rangle \in \mathcal{E}\left(R^{i}\right)$,

3. if $\left\langle v,>n, R^{i}\right\rangle \in \mathrm{Eq}$, then $\operatorname{Card}\left(R^{i}(\pi(v))\right)>n$,

4. if $\left\langle v,<n, R^{i}\right\rangle \in \mathrm{Eq}$, then $\operatorname{Card}\left(R^{i}(\pi(v))\right)<n$. 
We now show that as the derivation proceeds, we can extend our mapping $\pi$ not violating the conditions above.

Let $s_{0}$ be a node in $\mathrm{S}$ such that $\varphi \in \mathcal{L}^{\prime}\left(s_{0}\right)$. By (P0) from Definition 8.9 such a node exists. Let $v_{0}$ the initial node in $G$. We set $\pi\left(v_{0}\right)=s_{0}$. We show that no application of a rule can violate the conditions above. The rules $(\wedge),(\vee),\left(\square^{i}\right)$ and $\left(\square^{\tilde{i}}\right)$ follow the conditions due to their strict accordance with (P2)-(P5). We only need to choose a correct disjunct when $(V)$ is applied. (ch) non-deterministically decides whether a partition is empty or not, whenever a respective inequality is not present in Eq. Obviously, as we can check what is the case in T, we can follow it in ascribing inequalities to partitions. However, no condition written above can be violated by (ch). The application of the rules $\left(\diamond_{>n}^{i}\right)$ and $\left(\diamond_{<n}^{i}\right)$ to a formula $\psi$ at a node $v_{j}$ yields tuples $\left\langle v_{j},>n, R^{i}\right\rangle$ $\left(\left\langle v_{j},>n, R^{i}\right\rangle\right.$ respectively) added to Eq. It also indicates that formulas $\diamond_{>n}^{i} \psi \in \mathcal{L}^{\prime}\left(\pi\left(v_{j}\right)\right)\left(\nabla_{<n}^{i} \psi \in \mathcal{L}^{\prime}\left(\pi\left(v_{j}\right)\right)\right.$ respectively) and, what follows, $\operatorname{Card}\left(R^{i}\left(\pi\left(v_{j}\right)\right)\right)>n\left(\operatorname{Card}\left(R^{i}\left(\pi\left(v_{j}\right)\right)\right)<n\right)$. The conditions for $\pi$ are still preserved since there is no possibility of violating them by $\left(\nabla_{>n}^{i}\right)$ or $\left(\diamond_{<n}^{i}\right)$. If the (fil) rule is applied, it means that the solver provided us with a solution of a set of inequalities delivered to Eq by $\left(\nabla_{>n}^{i}\right)$, $\left(\diamond_{<n}^{i}\right)$ and $(\mathrm{ch})$. The solution returned by the solver is minimal. Since, by the assumption, $T$ is also minimal, the set-up of its nodes is also a minimal solution of respective inequalities (implied by $\nabla_{>n}^{i}$ and $\nabla_{<n}^{i}$ formulas present in $T$ ). We therefore choose the solution reflecting the set-up of our tableau. Afterwards, we deal with the non-deterministic part of (fil). It basically amounts to adding edges to distribute particular nodes between partitions that emerged from a non-empty partition. We do it in accordance with the set-up of nodes in $T$. The deterministic part of (fil) boils down to complementing partitions according to a solution provided by the solver. As indicated, the minimal solution reflects a distribution of nodes with respect to particular accessibility relations in T. After we exhaustively apply (fil), and suitably match respective nodes of $\mathbf{G}$ and $\mathrm{S}$, the last two conditions above are satisfied.

Since we showed that $\mathcal{L}(v) \subseteq \mathcal{L}^{\prime}(\pi(v))$, there is no possibility that a clash of the form $\{\psi, \neg \psi\} \in \mathcal{L}(v)$ for some $v \in V$ occurred in G. Also, it cannot be the case that at some stage of the arithmetical derivation there existed no non-negative solution for a set of inequalities from Eq. For it would mean that no distribution of accessibility relations' fillers 
was possible, which cannot be the case since it violates (P6) or (P7) from Definition 8.9. In picking a minimal solution of a set of inequalities we always followed the set-up of $\mathrm{T}$.

Hence, the calculus is complete.

THEOREM 8.20 (Termination). The hybrid algorithm for the logic $\mathcal{M}\left(\mathrm{E}_{n}\right)$ terminates.

Proof. Termination follows from the fact that whenever a formula $\varphi$ is satisfiable, a graph returned by the calculus is minimal (with respect to the size of the domain), and by Theorem 5.16 finite. The calculus does not contain shrinking rules that remove nodes from a graph. Consequently, no repetitive sequences of steps introducing and eliminating the same node are possible. As a result, all runs of applications of rules (before obtaining a clash or a complete, clash-free graph) are finite. The problem of linear integer programming is NP-complete, so the inequality solver always terminates. Hence, the conclusion.

Our external inequality solver not only deals with the arithmetical reasoning, but also, together with the finite model property of $\mathcal{M}\left(\mathrm{E}_{n}\right)$ serves as a blocking mechanism! The following example explores the formula $E_{=0}\left(E_{=0} \varphi\right)$ from Fig. 24, which yields the infinite derivation when no blocking mechanism is attached to the ordinary (non-hybrid) tableau calculus.

EXAmple 8.2. Let $\varphi=\mathrm{E}_{<1}\left(\mathrm{E}_{<1} p\right)$. We will use symbols $R^{1}$ and $R^{2}$ to abbreviate, respectively, $R^{\left(v_{0}, \mathrm{E}_{<1} p,<1\right)}$ and $R^{\left(v_{0}, p,<1\right)}$. We start the derivation in $v_{0}$ as the initial node of a graph. After rewriting we obtain

$$
\diamond_{<1}^{1}\left(\mathrm{E}_{<1} p\right) \wedge \square^{1}\left(\mathrm{E}_{<1} p\right) \wedge \square^{\tilde{1}} \neg\left(\mathrm{E}_{<1} p\right) \in \mathcal{L}\left(v_{0}\right),
$$

and

$$
\mathrm{p}_{R^{\varnothing}}<1 \in \mathrm{Eq} .
$$

After applying $\left(\nabla_{<n}^{i}\right)$, a tuple $\left\langle v_{0},<1, R^{1}\right\rangle$ is delivered to $\mathrm{R}$. The solver counts $\mathcal{P}(\mathrm{R})$, where $\mathrm{R}$ is a set of all accessibility relations currently occurring in Eq:

$$
\mathcal{P}(\mathrm{R})=\left\{\varnothing,\left\{R^{\varnothing}\right\},\left\{R^{1}\right\},\left\{R^{\varnothing}, R^{1}\right\}\right\} .
$$




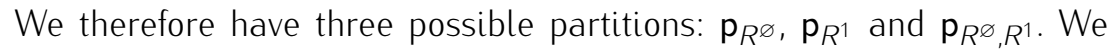
already have

$$
\mathrm{p}_{R^{\varnothing}}^{c}+\mathrm{p}_{R^{\varnothing}, R^{1}}^{c}>0 \text { and } \mathrm{p}_{R^{1}}^{c}+\mathrm{p}_{R^{\varnothing}, R^{1}}^{c}<1 \text {. }
$$

(ch) non deterministically adds

$$
\mathrm{p}_{R^{\varnothing}}^{c}>0, \mathrm{p}_{R^{1}}^{c}<1 \text { and } \mathrm{p}_{R^{\varnothing}, R^{1}}^{c}<1
$$

to Eq. The solver therefore returns the (unique) minimal solution:

$$
\sigma\left(\mathrm{p}_{R^{\varnothing}}\right)=1, \sigma\left(\mathrm{p}_{R^{1}}\right)=0 \quad \text { and } \quad \sigma\left(\mathrm{p}_{R^{\varnothing}, R^{1}}\right)=0 \text {. }
$$

The (fil) rule does not have to be employed. According to the solution provided by the solver, the only node that was an element of the old partition $\mathrm{p}_{R^{\varnothing}}$ is now ascribed to the new partition $\mathrm{p}_{R^{\varnothing}}$. No complement has to be made, since 1 indicated by $\sigma\left(\mathrm{p}_{R^{\varnothing}}\right)$ is already present. Subsequently, $\left(\square^{\tilde{i}}\right)$ is employed and propagates $\dot{\rightarrow} \mathrm{E}_{<1} p$ to all worlds that are not elements of $R^{1}\left(v_{0}\right)$. Since $R^{1}$ turned out to be empty, $\dot{\rightarrow} \mathrm{E}_{<1} \psi$ is distributed to all nodes in a graph, namely $v_{0}$. After rewriting we obtain

$$
\diamond_{<0}^{2} p \wedge \square^{2} p \in \mathcal{L}\left(v_{0}\right)
$$

Clearly, no clash occurs. Next, Eq $\longrightarrow$ Eq $\cup\left\{\left\langle v_{0},>0, R^{2}\right\rangle\right\}$. After recounting $\mathcal{P}(\mathrm{R})$ we obtain:

$$
\begin{aligned}
\mathcal{P}(\mathrm{R})= & \left\{\varnothing,\left\{R^{\varnothing}\right\},\left\{R^{1}\right\},\left\{R^{2}\right\},\left\{R^{\varnothing}, R^{1}\right\},\right. \\
& \left.\left\{R^{\varnothing}, R^{2}\right\},\left\{R^{1}, R^{2}\right\},\left\{R^{\varnothing}, R^{1}, R^{2}\right\}\right\} .
\end{aligned}
$$

So far we know that

$$
\begin{aligned}
& \mathrm{p}_{R^{\varnothing}}^{c}+\mathrm{p}_{R^{\varnothing}, R^{1}}^{c}+\mathrm{p}_{R^{\varnothing}, R^{2}}^{c}+\mathrm{p}_{R^{\varnothing}, R^{1}, R^{2}}^{c}>0, \\
& \mathrm{p}_{R^{1}}^{c}+\mathrm{p}_{R^{\varnothing}, R^{1}}^{c}+\mathrm{p}_{R^{1}, R^{2}}^{c}+\mathrm{p}_{R^{\varnothing}, R^{1}, R^{2}}^{c}<1, \\
& \mathrm{p}_{R^{2}}^{c}+\mathrm{p}_{R^{\varnothing}, R^{2}}^{c}+\mathrm{p}_{R^{1}, R^{2}}^{c}+\mathrm{p}_{R^{\varnothing}, R^{1}, R^{2}}^{c}>0 .
\end{aligned}
$$

The (ch) rule non-deterministically adds

$$
\begin{gathered}
\mathrm{p}_{R^{\varnothing}}^{c}<1, \quad \mathrm{p}_{R^{1}}^{c}<1, \quad \mathrm{p}_{R^{2}}^{c}<1, \quad \mathrm{p}_{R^{\varnothing}, R^{1}}^{c}<1, \\
\mathrm{p}_{R^{\varnothing}, R^{2}}^{c}>0, \quad \mathrm{p}_{R^{1}, R^{2}}^{c}<1, \quad \mathrm{p}_{R^{\varnothing}, R^{1}, R^{2}}^{c}<1
\end{gathered}
$$


to Eq. The inequality solver returns the (again unique) minimal solution:

$$
\begin{gathered}
\sigma\left(\mathrm{p}_{R^{\varnothing}}\right)=0, \quad \sigma\left(\mathrm{p}_{R^{1}}\right)=0, \quad \sigma\left(\mathrm{p}_{R^{2}}\right)=0, \quad \sigma\left(\mathrm{p}_{R^{\varnothing}, R^{1}}\right)=0, \\
\sigma\left(\mathrm{p}_{R^{\varnothing}, R^{2}}\right)=1, \quad \sigma\left(\mathrm{p}_{R^{1}, R^{2}}\right)=0, \quad \sigma\left(\mathrm{p}_{R^{\varnothing}, R^{1}, R^{2}}\right)=0 .
\end{gathered}
$$

The non-deterministic part of (fil) does not have to be employed, as there is only one world that, according to the calculations of the solver, has to be transferred from $\mathrm{p}_{R^{\varnothing}}$ to $\mathrm{p}_{R^{\varnothing}, R^{2}}$ resulting from a division of $\mathrm{p}_{R^{\varnothing}}$. This is done by adding $R^{2}$ to $\mathcal{L}\left(\left\langle v_{0}, v_{0}\right\rangle\right)$. The $\left(\square^{i}\right)$ does the rest of the job by propagating $p$ to all $R^{2}$-fillers of $v_{0}$, namely $v_{0}$ itself. Since no other rule is applicable, we obtain a complete, clash free graph for $\varphi$ only consisting of one node $v_{0}$ and one edge $\left\langle v_{0}, v_{0}\right\rangle$ with $R^{2} \in \mathcal{L}\left(\left\langle v_{0}, v_{0}\right\rangle\right)$.

The presented calculus is, to the best of our knowledge, the first hybrid calculus for a modal logic with global counting operators that involves external arithmetic reasoning. Faddoul and Haarslev ([30]) establish an algorithm for the logic $\mathcal{S H O Q}$ which involves some implicit form of global counting under the guise of nominals, however no such calculus was elaborated for the logic with explicit global counting modalities. The inspiration for the calculus presented in this section was the one for the logic $\mathcal{A L C Q}$ found in [31], nevertheless several serious changes had to be introduced to the algorithm to make it capable of dealing with global cardinality constraints.

We postpone some issues for future research. For instance, worth scrutinising is the question whether the non-deterministic part of the (fil) rule can be refined so that the the number of possible moves decreases. A similar question applies to the inequality solver that nondeterministically returns a minimal solution for a set of inequalities.

\subsection{CONCLUDING REMARKS}

The presentation of tableau calculi for the logic $\mathcal{M}\left(\mathrm{E}_{n}\right)$ in this chapter shows that there is a wide plethora of possible approaches. The tableau calculus $\mathcal{T}_{\mathcal{M}\left(\mathrm{E}_{n}\right)}^{P}$, the major part of the chapter is devoted to, is the example of a rather elegant and conceptually simple algorithm that is based on the ingenious idea of introducing as an explicit rule 
some form of analytical cut which serves as a blocking mechanism. This brings certain profits like transparency of the calculus, generation of minimal models from open and fully expanded branches of tableaux or adaptability to additional frame conditions introduced as explicit rules. Unfortunately, it also causes some trouble, mainly regarding the large branching factor implied by the unrestricted blocking splitting rule. $\mathcal{T}_{\mathcal{M}\left(\mathrm{E}_{n}\right)}^{p}$, though, remains complexity optimal. Novel in our considerations is the refinement that results in an internalized version $\mathcal{T}_{\mathcal{M}\left(\mathrm{E}_{n}\right)}^{i}$ of $\mathcal{T}_{\mathcal{M}\left(\mathrm{E}_{n}\right)}^{p}$ in which the rules are defined in the language of the object logic. This is possible as $\mathcal{M}\left(E_{n}\right)$ is expressive enough to define its own semantics. Interestingly, the termination proof for $\mathcal{T}_{\mathcal{M}\left(\mathrm{E}_{n}\right)}^{p}$ relies on the finite model property of the logic $\mathcal{M}\left(E_{n}\right)$ whereas for most other calculi the finite model property of a logic follows as a consequence from a termination proof. Possible directions for future work involve specialising the unrestricted blocking mechanism so it deals with counting operators more efficiently.

Other algorithms described in this chapter also bring some benefits. Kaminski's et al. system involving the pattern-based blocking mechanism proves very efficient in performance (see [60, 40]), albeit its extension on cases of other frame classes is not straightforward. Horrocks' et al. algorithm presents a constructive approach to tableaux. In terms of complexity, it does not yield the best results (however, one must remember that originally it was established for much more powerful logics which often even lack the finite model property) but it introduces an interesting tool to deal with accessibility relation inclusions, namely non-deterministic finite automata. They allow keeping track of these inclusions in an abstract and elegant way. The main advantage of the hybrid algorithm from Section 8.6.3 is externalising the arithmetical part of a reasoning within a tableau ${ }^{12}$. Due to the global character of cardinality constraints some additional factor of the non-determinism is involved when compared to the algorithm for $\mathcal{A L C Q}$ from [31], which puts in question the advantages in performance over non-hybrid algo-

12 In fact, we conjecture that the technique involving an inequality solver and arithmetical reasoning, rather than traditional blocking mechanisms, in potentially infinite derivations, when slightly modified could also be applied to the cases of other modal logics, not involving counting operators. 
rithms for $\mathcal{M}\left(\mathrm{E}_{n}\right)$. However, an extra profit of separating arithmetical reasoning is superfluity of any form of blocking, even though in other approaches it is indispensable to avoid infinite derivations. Investigating whether any reduction of non-determinism factor in the calculus is possible is a matter of future research. 



\section{SUMMARY}

\subsection{RESULTS}

The original results of this book split into two parts. This division reflects the division of the book itself. We can say that the first type of results concern model-theoretic and complexity properties of hybrid logics. Since hybrid logics which we call standard are quite well investigated, our efforts focused on hybrid logics referred to as non-standard in this book. By non-standard hybrid logics, we understand modal logics with global counting operators $\left(\mathcal{M}\left(E_{n}\right)\right)$ whose expressive power matches the expressive power of binder-free standard hybrid logics. The relevant results comprise:

1. Establishing a sound and complete axiomatization for the modal logic $K$ with global counting operators $\left(\mathcal{M}_{K}\left(E_{n}\right)\right)$, which can be easily extended onto other frame classes,

2. Establishing tight complexity bounds, namely NEXPTIME-completeness for the modal logic with global counting operators defined over the classes of arbitrary, reflexive, symmetric, serial and transitive frames $\left(\mathcal{M}_{K}\left(E_{n}\right), \mathcal{M}_{\top}\left(E_{n}\right), \mathcal{M}_{\mathrm{D}}\left(E_{n}\right), \mathcal{M}_{\mathrm{B}}\left(E_{n}\right), \mathcal{M}_{K 4}\left(E_{n}\right)\right)$ with numerical subscripts coded in binary. Establishing the exponential-size model property for logics defined over the classes of Euclidean and equivalential frames $\left(\mathcal{M}_{\mathrm{K} 5}\left(\mathrm{E}_{n}\right), \mathcal{M}_{\mathrm{S} 5}\left(\mathrm{E}_{n}\right)\right)$. In the second case, we currently lack complexity bounds which are tight. We only conjecture that the satisfiability problem for the modal logic with global counting operators over Euclidean and equivalential frames is NP-complete. Nevertheless, the computational complexity of non-standard hybrid logics turned out to be rather fixed and less dependant on the frame class it is defined over than standard hybrid logics.

Results of the second type consist of designing concrete deductive (tableau and sequent) systems for standard and non-standard hybrid logics. More precisely, they include: 
1. Devising a prefixed and an internalized tableau calculi which are sound, complete and terminating for a rich class of binder-free standard hybrid logics. An interesting feature of the indicated calculi is the nonbranching character of the rule $(\neg D)$,

2. Devising a prefixed and an internalized tableau calculi which are sound, complete and terminating for non-standard hybrid logics. The internalization technique applied to a tableau calculus for the modal logic with global counting operators is novel in the literature,

3. Devising the first hybrid algorithm involving an inequality solver for modal logics with global counting operators. Transferring the arithmetical part of reasoning to an inequality solver turned out to be sufficient for ensuring termination.

\subsection{FUTURE RESEARCH}

Three main topics emerge that should be a subject of our future research:

1. Research on the complexity theory for modal logics with global counting operators. Although certain results are established, there are still many blank spots left on the road-map. Tight complexity bounds should be established for these logics defined over Euclidean and equivalential frames. Further frame classes, like (the class of) transitive trees, linear orderings, or frames based on natural numbers, should be thoroughly investigated.

2. Broadening the scope of applicability of the unrestricted blocking rule as a blocking mechanism. The current condition that all underlying logics must satisfy, the finite model property, seems to be too strict, thus making the (ub) rule useless as a blocking mechanism for many decidable logics which do not enjoy this property. Loosening the condition to the finite quasi-structure property seems fair, especially that, as we strongly conjecture, most of the decidable hybrid logics lacking the finite model property have some form of the finite quasistructure property. It would require reinventing the whole construction of a tableau calculus which for each satisfiable formula would have 
to yield a quasi-structure rather than a model. In that case the (ub) rule would compare between the elements of such a quasi-structure. A good example of a tableau decision procedure which for each satisfiable formula returns a mosaic (rather than a model) is a calculus of Wolter, Zakharyaschev et al. designed for modal description logics in $[100,101,64]$.

3. Refinement of the hybrid calculus for modal logics with global counting operators. Currently, the calculus is designed in such a way that it does not need any particular blocking mechanism since termination is ensured by an appropriately performed arithmetical reasoning. However, in the present version of the calculus the non-determinism factor is quite high, which negatively affects practical performance of such an algorithm. It should be checked whether decreasing the level of nondeterminism involved is possible. 

[1] MetTEL website. http://mettel - prover .org.

[2] James Allen. Towards a general theory of action and time. Artificial Intelligence, 23(2):123-154, 1984.

[3] Carlos Areces, Patrick Blackburn, and Maarten Marx. A roadmap on complexity for hybrid logics. In J. Flum and M. RodríguezArtalejo, editors, CSL, volume 1683 of Lecture Notes in Computer Science, pages 307-321. Springer, 1999.

[4] Carlos Areces, Patrick Blackburn, and Maarten Marx. The computational complexity of hybrid temporal logics. Logic Journal of the IGPL, 8(5):653-679, 2000.

[5] Carlos Areces, Patrick Blackburn, and Maarten Marx. Hybrid logics: Characterization, interpolation and complexity. Journal of Symbolic Logic, 66(3):977-1010, 2001.

[6] Carlos Areces, Guillaume Hoffmann, and Alexandre Denis. Modal logics with counting. In Dawar and de Queiroz [27], pages 98-109.

[7] Guillaume Aucher, Bastien Maubert, and François Schwarzentruber. Tableau method and NEXPTIME-completeness of DELsequents. Electronic Notes in Theoretical Computer Science, 278:17-30, 2011.

[8] Franz Baader, Diego Calvanese, Deborah L. McGuinness, Daniele Nardi, and Peter F. Patel-Schneider, editors. The description logic handbook: theory, implementation, and applications. Cambridge University Press, New York, NY, USA, 2003.

[9] Patrick Blackburn. Representation, reasoning, and relational structures: a hybrid logic manifesto. Logi Journal of the IGPL, 8 (3):339-365, 2000. 
[10] Patrick Blackburn. Internalizing labelled deduction. Journal of Logic and Computation, 10(1):137-168, 2000.

[11] Patrick Blackburn. Arthur prior and hybrid logic. Synthese, 150 (3):329-372, 2006.

[12] Patrick Blackburn and Jerry Seligman. Hybrid languages. Journal of Logic Languaga and Information, 4(3):251-272, 1995.

[13] Patrick Blackburn and Jerry Seligman. What are hybrid languages? In M. Kracht, M. de Rijke, H. Wansing, and M. Zakharyaschev, editors, Advances in Modal Logic, volume 1, pages 41-62. CSLI Publications, 1998.

[14] Patrick Blackburn and Balder ten Cate. Pure extensions, proof rules, and hybrid axiomatics. Studia Logica, 84(2):277-322, 2006.

[15] Patrick Blackburn, Maarten de Rijke, and Yde Venema. Modal logic. Cambridge University Press, New York, NY, USA, 2001.

[16] Thomas Bolander and Patrick Blackburn. Termination for hybrid tableaus. Journal of Logic and Computation, 17(3):517-554, 2007.

[17] Thomas Bolander and Patrick Blackburn. Terminating tableau calculi for hybrid logics extending k. Electronic Notes in Theoretical Computer Science, 231:21-39, 2009.

[18] Thomas Bolander and Torben Braüner. Two tableau-based decision procedures for hybrid logic. In H. Schlingloff, editor, 4th Methods for Modalities Workshop (M4M), Informatik-Bericht Nr. 194, pages 79-96. Humboldt-Universität zu Berlin, 2005.

[19] Thomas Bolander and Torben Braüner. Tableau-based decision procedures for hybrid logic. Journal of Logic and Computation, 16(6):737-763, 2006.

[20] Torben Braüner. Two natural deduction systems for hybrid logic: A comparison. Journal of Logic, Language and Information, 13 (1):1-23, 2004. 
[21] Torben Braüner. Hybrid logic and its proof-theory. Springer, Dordrecht, Netherlands, 2011.

[22] Robert Bull. An approach to tense logic. Theoria, 36(3):282-300, 1970.

[23] Francesco Caro. Graded modalities II (canonical models). Studia Logica, 47(1):1-10, 1988.

[24] Balder ten Cate. Model theory for extended modal languages. PhD thesis, University of Amsterdam, 2005.

[25] Claudio Cerrato. Decidability by filtrations for graded normal logics (graded modalities V). Studia Logica, 53(1):61-73, 1994.

[26] Marcello D'Agostino. Tableau methods for classical propositional logic. In M. D’Agostino, D. Gabbay, R. Hahnle, and J. Posegga, editors, Handbook of Tableau Methods, pages 45-123. Kluwer Academic Publishers, Dordrecht, Netherlands, 1999.

[27] A. Dawar and R. de Queiroz, editors. Logic, Language, Information and Computation, 17th International Workshop, WoLLIC 2010, Brasilia, Brazil, July 6-9, 2010. Proceedings, volume 6188 of Lecture Notes in Computer Science, 2010. Springer.

[28] Maarten de Rijke. The modal logic of inequality. Journal of Symbolic Logic, 57(2):566-584, 1992.

[29] Stéphane Demri. A simple tableau system for the logic of elsewhere. In P. Miglioli, U. Moscato, D. Mundici, and M. Ornaghi, editors, TABLEAUX, volume 1071 of Lecture Notes in Computer Science, pages 177-192. Springer, 1996. ISBN 3-540-61208-4.

[30] Jocelyne Faddoul and Volker Haarslev. Algebraic tableau reasoning for the description logic $\mathcal{S H O Q}$. Journal of Applied Logic, 8 (4):334-355, 2010.

[31] Jocelyne Faddoul, Nasim Farsinia, Volker Haarslev, and Ralf Möller. A hybrid tableau algorithm for $\mathcal{A L C Q}$. In F. Baader, C. Lutz, and B. Motik, editors, Description Logics, volume 353 of 
CEUR Workshop Proceedings, pages 725-726. CEUR-WS.org, 2008.

[32] Maurizio Fattorosi-Barnaba and Francesco Caro. Graded modalities I. Studia Logica, 44(2):197-221, 1985.

[33] Kit Fine. In so many possible worlds. Notre Dame Journal of Formal Logic, 13(4):516-520, 1972.

[34] Melvin Fitting. Modal proof theory. In P. Blackburn, F. Wolter, and J. van Benthem, editors, Handbook of Modal Logic, pages 85-138. Elsevier, Amsterdam, 2006.

[35] Dov Gabbay. Labelled Deductive Systems. Oxford University Press, New York, NY, USA, 1996.

[36] Jean Gallier. Logic for computer science: foundations of automatic theorem proving. Harper \& Row Publishers, Inc., New York, NY, USA, 1985.

[37] George Gargov and Valentin Goranko. Modal logic with names. Journal of Philosophical Logic, 22:607-636, 1993.

[38] Stefan Göller, Arne Meier, Martin Mundhenk, Thomas Schneider, Michael Thomas, and Felix Weiß. The complexity of monotone hybrid logics over linear frames and the natural numbers. In T. Bolander, T. Braüner, S. Ghilardi, and L. Moss, editors, Advances in Modal Logic, pages 261-278. College Publications, 2012.

[39] Valentin Goranko. Hierarchies of modal and temporal logics with reference pointers. Journal of Logic, Language and Information, 5(1):1-24, 1996.

[40] Daniel Götzmann, Mark Kaminski, and Gert Smolka. Spartacus: A tableau prover for hybrid logic. Electronic Notes in Theoretical Computer Science, 262:127-139, 2010.

[41] Erich Grädel, Phokion Kolaitis, and Moshe Vardi. On the decision problem for two-variable first-order logic. Bulletin of Symbolic Logic, 3(1):53-69, 1997. 
[42] Guillaume Hoffmann. Lightweight hybrid tableaux. Journal of Applied Logic, 8(4):397-408, 2010.

[43] lan Horrocks and Ulrike Sattler. A description logic with transitive and inverse roles and role hierarchies. Journal of Logic and Computation, 9(3):385-410, 1999.

[44] Ian Horrocks and Ulrike Sattler. Decidability of $\mathcal{S H \mathcal { Q }}$ with complex role inclusion axioms. In G. Gottlob and T. Walsh, editors, IJCAI, pages 343-348. Morgan Kaufmann, 2003.

[45] Ian Horrocks and Ulrike Sattler. Decidability of $\mathcal{S H \mathcal { Q }}$ with complex role inclusion axioms. Artificial Intelligence, 160(1-2): 79-104, 2004.

[46] Ian Horrocks and Ulrike Sattler. A tableaux decision procedure

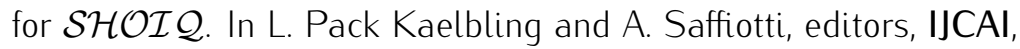
pages 448-453. Professional Book Center, 2005.

[47] Ian Horrocks and Ulrike Sattler. A tableau decision procedure

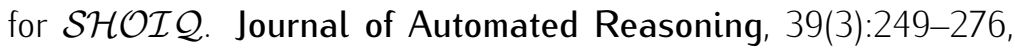
2007.

[48] Ian Horrocks, Ulrike Sattler, and Stephan Tobies. Practical reasoning for expressive description logics. In $\mathrm{H}$. Ganzinger, D. McAllester, and A. Voronkov, editors, LPAR, volume 1705 of Lecture Notes in Computer Science, pages 161-180. Springer, 1999.

[49] Ian Horrocks, Ulrike Sattler, and Stephan Tobies. Reasoning with individuals for the description logic $\mathcal{S H \mathcal { I }}$. In McAllester [65], pages 482-496.

[50] Ian Horrocks, Oliver Kutz, and Ulrike Sattler. The irresistible

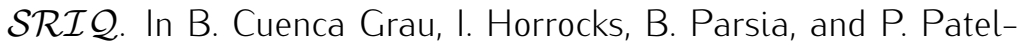
Schneider, editors, OWLED, volume 188 of CEUR Workshop Proceedings, pages 1-11. CEUR-WS.org, 2005.

[51] Ian Horrocks, Oliver Kutz, and Ulrike Sattler. The even more irresistible $\mathcal{S R O \mathcal { Q }}$. In P. Doherty, J. Mylopoulos, and Ch. Welty, editors, KR, pages 57-67. AAAI Press, 2006. 
[52] Andrzej Indrzejczak. Modal hybrid logic. Logic and Logical Philosophy, 16(2-3):147-257, 2007.

[53] Andrzej Indrzejczak. Natural Deduction, Hybrid Systems and Modal Logics. Springer, Dordrecht, Netherlands, 2010.

[54] Andrzej Indrzejczak and Michat Zawidzki. Decision procedures for some strong hybrid logics. Logic and Logical Philosophy, 22 (4), 2013. ISSN 2300-9802. URL http://wydawnictwoumk.pl/ czasopisma/index. php/LLP/article/view/LLP. 2013.022.

[55] Mark Kaminski. Incremental Decision Procedures for Modal Logics with Nominals and Eventualities. PhD thesis, Saarland University, 2012.

[56] Mark Kaminski and Gert Smolka. Terminating tableau systems for hybrid logic with difference and converse. Journal of Logic, Language and Information, 18(4):437-464, 2009.

[57] Mark Kaminski and Gert Smolka. Terminating tableau systems for hybrid logic with difference and converse. Journal of Logic, Language and Information, 18(4):437-464, 2009.

[58] Mark Kaminski and Gert Smolka. Terminating tableaux for hybrid logic with eventualities. In J. Giesl and R. Hähnle, editors, IJCAR, volume 6173 of Lecture Notes in Computer Science, pages 240254. Springer, 2010.

[59] Mark Kaminski, Sigurd Schneider, and Gert Smolka. Terminating tableaux for graded hybrid logic with global modalities and role hierarchies. In M. Giese and A. Waaler, editors, TABLEAUX, volume 5607 of Lecture Notes in Computer Science, pages 235249. Springer, 2009.

[60] Mark Kaminski, Sigurd Schneider, and Gert Smolka. Terminating tableaux for graded hybrid logic with global modalities and role hierarchies. Logical Methods in Computer Science, 7(1):1-21, 2011.

[61] Yevgeny Kazakov and lan Pratt-Hartmann. A note on the complexity of the satisfiability problem for graded modal logics. In 
LICS, pages 407-416. IEEE Computer Society, 2009. ISBN 9780-7695-3746-7.

[62] Mohammad Khodadadi, Renate Schmidt, Dmitry Tishkovsky, and Michat Zawidzki. Terminating tableau calculi for modal logic $k$ with global counting operators. 2012. URL http://www. mettel-prover. org/papers/KEn12. pdf.

[63] Saul Kripke. A completeness theorem in modal logic. Journal of Symbolic Logic, 24(1):1-14, 1959.

[64] Carsten Lutz, Holger Sturm, Frank Wolter, and Michael Zakharyaschev. A tableau decision algorithm for modalized $\mathcal{A L C}$ with constant domains. Studia Logica, 72(2):199-232, 2002.

[65] D. McAllester, editor. Automated Deduction - CADE-17, 17th International Conference on Automated Deduction, Pittsburgh, PA, USA, June 17-20, 2000, Proceedings, volume 1831 of Lecture Notes in Computer Science, 2000. Springer.

[66] Arne Meier, Martin Mundhenk, Thomas Schneider, Michael Thomas, Volker Weber, and Felix Weiss. The complexity of satisfiability for fragments of hybrid logic - part I. Journal of Applied Logic, 8(4):409-421, 2010.

[67] Martin Mundhenk and Thomas Schneider. Undecidability of multi-modal hybrid logics. Electronic Notes in Theoretical Computer Science, 174(6):29-43, 2007.

[68] Martin Mundhenk and Thomas Schneider. The complexity of hybrid logics over equivalence relations. Journal of Logic, Language and Information, 18(4):493-514, 2009.

[69] Martin Mundhenk, Thomas Schneider, Thomas Schwentick, and Volker Weber. Complexity of hybrid logics over transitive frames. Journal of Applied Logic, 8(4):422-440, 2010.

[70] Robert Myers and Dirk Pattinson. Hybrid logic with the difference modality for generalisations of graphs. Journal of Applied Logic, 8(4):441-458, 2010. 
[71] Sara Negri and Jan von Plato. Structural proof theory. Cambridge University Press, Cambridge, UK, 2001.

[72] Hans Jürgen Ohlbach and Jana Koehler. Role hierarchies and number restrictions. In R. Brachman et. al., editor, Description Logics, volume 410 of URA-CNRS, pages 1-5, 1997.

[73] Christos Papadimitriou. On the complexity of integer programming. Journal of ACM, 28(4):765-768, 1981.

[74] Christos Papadimitriou. Computational complexity. Academic Internet Publ., 2007.

[75] Christos Papadimitriou and Kenneth Steiglitz. Combinatorial optimization: algorithms and complexity. Prentice-Hall, Inc., Upper Saddle River, NJ, USA, 1982.

[76] Rohit Parikh. The completeness of propositional dynamic logic. In J. Winkowski, editor, Mathematical Foundations of Computer Science 1978, volume 64 of Lecture Notes in Computer Science, pages 403-415. Springer Berlin Heidelberg, 1978.

[77] Solomon Passy and Tinko Tinchev. Quantifiers in combinatory pdl: completeness, definability, incompleteness. In L. Budach, editor, FCT, volume 199 of Lecture Notes in Computer Science, pages 512-519. Springer, 1985.

[78] Solomon Passy and Tinko Tinchev. An essay in combinatory dynamic logic. Information and Computation, 93(2):263-332, 1991.

[79] Ian Pratt-Hartmann. The two-variable fragment with counting revisited. In Dawar and de Queiroz [27], pages 42-54.

[80] Arthur Prior. Past, Present and Future. Oxford University Press, New York, NY, USA, 1967.

[81] Arthur Prior. Papers on Time and Tense. Oxford University Press, New York, NY, USA, 2003.

[82] Renate Schmidt. Synthesising terminating tableau calculi for relational logics (invited paper). In H. de Swart, editor, RAMICS, 
volume 6663 of Lecture Notes in Computer Science, pages 4049. Springer, 2011

[83] Renate Schmidt and Dmitry Tishkovsky. Using tableau to decide description logics with full role negation and identity. Manuscript, available at http://www. mettel-prover. org/papers/ALB0id.pdf, 2011.

[84] Renate Schmidt and Dmitry Tishkovsky. Automated synthesis of tableau calculi. Logical Methods in Computer Science, 7(2): 1-32, 2011.

[85] Thomas Schneider. The Complexity of Hybrid Logics over Restricted Classes of Frames. PhD thesis, University of Jena, 2007.

[86] Jerry Seligman. A cut-free sequent calculus for elementary situated reasoning. Technical Report HCRC-RP 22, HCRC, Edinburgh, 1991.

[87] Jerry Seligman. Situated consequence in elementary situation theory. Technical Report IULG-92-16, Indiana University, 1992.

[88] Jerry Seligman. Internalization: The case of hybrid logics. Journal of Logic and Computation, 11(5):671-689, 2001.

[89] Raymond Smullyan. First-order logic. Dover Publications, Toronto, ON, Canada, 1995.

[90] Dmitry Tishkovsky, Renate Schmidt, and Mohammad Khodadadi. METTER: Towards a prover generation platform (system description). Manuscript, available at http://www. mettel-prover. org/papers/MetTeL2SysDesc. pdf, 2012.

[91] Stephan Tobies. The complexity of reasoning with cardinality restrictions and nominals in expressive description logics. Journal of Artificial Intelligence Research, 12:199-217, 2000.

[92] Stephan Tobies. Complexity Results and Practical Algorithms for Logics in Knowledge Representation. PhD thesis, RWTH Aachen, 2001. 
[93] Miroslava Tzakova. Tableau calculi for hybrid logics. In N. Murray, editor, TABLEAUX, volume 1617 of Lecture Notes in Computer Science, pages 278-292. Springer, 1999.

[94] Johan van Benthem. Modal Correspondence Theory. PhD thesis, Universiteit van Amsterdam, 1977.

[95] Johan van Benthem. Modal frame classes revisited. Fundamenta Informaticae, 18:307-317, 1993.

[96] Johan Van Benthem. Modal Logic for Open Minds. University of Chicago Press, Chicago, IL, USA, 2010.

[97] Wiebe van der Hoek and Maarten de Rijke. Generalized quantifiers and modal logic. Journal of Logic, Language and Information, 2:19-58, 1993.

[98] Wiebe van der Hoek and Maarten de Rijke. Counting objects. Journal of Logic and Computation, 5(3):325-345, 1995.

[99] Peter van Emde Boas. The convenience of tiling. In Andrea Sorbi, editor, Complexity, Logic and Recursion Theory, volume 187 of Lecture Notes in Pure and Applied Mathematics, pages 331-363. Marcel Dekker Inc., 1997.

[100] Frank Wolter and Michael Zakharyaschev. On the decidability of description logics with modal operators. In A. Cohn, L. Schubert, and S. Shapiro, editors, KR, pages 512-523. Morgan Kaufmann, 1998.

[101] Frank Wolter and Michael Zakharyaschev. Modal description logics: Modalizing roles. Fundamenta Informaticae, 39(4):411438, 1999.

[102] Michat Zawidzki. Adequacy of the logic $K\left(E_{n}\right)$. Bulletin of the Section of Logic, 43(3-4):155-172, 2012.

[103] Michat Zawidzki. Tableau-based decision procedure for hybrid logic with satisfaction operators, universal modality and difference modality. In R. Rendsvig and S. Katrenko, editors, ESSLLI, 
volume 954 of Workshop Proceedings, pages 191-201. CEUR, 2012.

[104] Michat Zawidzki, Renate Schmidt, and Dmitry Tishkovsky. Satisfiability problem for modal logic with global counting operators coded in binary is NEXPTIME-complete. Information Processing Letters, 113(1-2):34-38, 2013. 



\section{INDEX}

(t) condition, 109

MetTeL, 151, 152

Agostino (de), M., 120

Allen, J., 37

analytical cut, 103, 111, 113, 192

Areces, C., 1, 2, 38, 84

atomic concept, 87

atomic decomposition, 176

background theory, 98, 138

Benthem, J. van, 10, 28

binder, 24

Blackburn, P., 1, 2, 9, 20, 37, 38, $95,110,126,133$

blocking, 4, 21, 126

ancestor, 132

equality, 131

everywhere, 133

pairwise, 169

pattern-based, 132, 157

subset, 128, 129

unrestricted, 4, 103, 119, 134, 141

Bolander, T., 38, 95, 110, 126, 133

bounded tiling problem, 15, 59

exponential, 60, 73

branch, 154

closed, 103

evident, 155

fully-expanded, 103 open, 103

pre-evident, 160

quasi-evident, 158

branching factor, 103, 108, 111, 120, 126

Braüner, T., 2, 38, 94, 95, 126, 133

Bull, R., 37

canonical model, 52

canonical projection valuation, 104 , 140

Caro, F., 45, 84

Cate, B. ten, 37

Cerrato, C., 84

clash, 169, 181

closure, 162

co-problem, 12

colon notation, 149

completeness

of a Hilbert-style calculus, 45

of a tableau calculus, 21, 103

completion graph, 168, 181

complexity class

EXPTIME, 13

NEXPTIME, 13

NP, 13

NeXPTime, 13

PSPACE, 13

$P, 13$ 
hardness, 14

hierarchy, 13

membership, 14

computational complexity, 12

of $\mathcal{M}_{\mathrm{B}}\left(\mathrm{E}_{n}\right), 66$

of $\mathcal{M}_{\mathrm{D}}\left(\mathrm{E}_{n}\right), 71$

of $\mathcal{M}_{\mathrm{S} 5}\left(\mathrm{E}_{n}\right), 82$

of $\mathcal{M}_{\mathrm{K} 5}\left(\mathrm{E}_{n}\right), 82$

of $\mathcal{M}_{\mathrm{K}}\left(\mathrm{E}_{n}\right), 59$

of $\mathcal{H}(\mathrm{E}), 35$

of $\mathcal{H}_{K}(\diamond, \downarrow), 35$

of $\mathcal{H}_{K}(@), 35$

of $\mathcal{M}_{\mathrm{T}}\left(\mathrm{E}_{n}\right), 64$

of $\mathcal{M}_{\mathrm{K} 4}\left(\mathrm{E}_{n}\right), 72$

of temporal logic with nominals, 35

conservative extension, 42

constructive completeness, 103, 104, 155

decidability, 12

decision procedure, 12, 115, 145

deductive system, 17

description logic, 87

$\mathcal{A L C Q}, 176$

$\mathcal{S H I} \mathcal{I}, 161$

$\mathcal{S H O Q}, 152,176$

domain sort, 97, 99, 147

domain sort internalization, 112

equational closure, 132, 154

Existence Lemma, 53

expressive power

hierarchy, 29

of $\mathcal{M}\left(E_{n}\right), 41$

of binder-free standard hybrid logics, 27
Faddoul, J., 85, 191

Fattorosi-Barnaba, M., 45, 84

filler, 176

Fine, K., 84

formula

evident, 155

quasi-evident, 158

frame condition

first-order, 27

second-order, 28

function $\lambda, 48$

Gabbay, D., 20

Gargov, G., 37

general frame, 31

compact, 31

descriptive, 31

differentiated, 31

tight, 31

two-sorted, 31

two-sorted descriptive, 32

two-sorted strongly descriptive, 32

Gentzen, G., 18

global satisfiability problem, 12

Goranko, V., 37

grid, 59

grid-like structure, 64, 67, 72,

77

Götzmann, D., 134

Haarslev, V., 191

Hilbert-style calculus

for $\mathcal{M}_{\mathrm{K}}\left(\mathrm{E}_{n}\right), 42$

for $\mathcal{H}_{K}(\mathrm{D}), 31$

for $\mathcal{H}_{\mathrm{K}}, 32$

for $\mathcal{H}_{K}(@), 32$ 
for $\mathcal{H}_{K}(\mathrm{E}), 33$

Hoek, W. van der, 84

Horrocks, I., 84, 192

hybrid calculus, 191

hybrid logic

non-standard, 42

standard, 23

hybrid translation, 29, 147

hybridization, 36

individual, 89

Indrzejczak, A., 17, 38, 94

inequality solver, 182

interpretation, 87

Kaminski, M., 19, 38, 95, 132$134,152,153,157,192$

Kazakov, Y., 73

knowledge base, 88

Kripke, S., 9

lazy branching, 133

Lindenbaum's Lemma, 47

many-sorted first-order language, 96-98, 136

Marx, M., 1, 38

maximal consistent set, 45

merging, 172

mirrored-copy, 166

modal description logic, 197

modal logic, 9

modality

difference, 30

global counting, 39, 84

graded, 39, 84, 178

universal, 30, 37

model branch induced, 104, 151

Kripke, 9

model checking problem, 12

model property

exponential size, 15

exponential-size, 83, 146

finite, 57, 64, 66, 71, 72, 82, 124,157

polysize, 83, 126, 146

tree, 169

Mondadori, M., 120

Mundhenk, M., 38

Myers, R., 119

negation-normal form, 153

Negri, S., 119

nominal, 23, 26

non-deterministic finite automaton, 163, 165, 167

non-deterministic finite pre-automaton, 165

number coding

binary, 40

unary, 40

operator

$\downarrow, 26$

$\exists, 27$

Holds, 37

satisfaction, 23, 27

singleton set, 96

partition, 181, 182

Passy, S., 37

path, 173

pattern, 132, 157

Pattinson, D., 119

Plato, J. von, 119 
polynomial time reduction, 15 possible world propositions, 36

Pratt-Hartmann, I., 73

prefix, 95

Prior, A., 1, 36, 37

pruning, 172

reflection

of a branch, 104, 105

of a formula, 104

rewriting, 177

Rijke (de), M., 9

role, 87

role inclusion, 88

rule refinement, 108, 109, 146

satisfaction relation, 10

satisfiability problem, 11

satisfying family of an MCS, 51

Schmidt, R., 4, 110

Schneider, S., 152

Schneider, T., 1, 36, 38

Seligman, J., 1, 2, 37, 38

sequent, 18

sequent calculus, 18

$$
\mathcal{S}_{\mathcal{H}_{\text {b-f }}, 118}
$$

sequent rule, 18

simple accessibility relation, 172

sink point, 77

Skolem

function, 101, 113

functions, 149

term, 101, 149

Skolemization, 98

Smolka, G., 38, 95, 132-134, 152

Smullyan, R., 19

sort, 96, 136 soundness

of a Hilbert-style calculus, 43

of a tableau calculus, 21, 103

standard translation, 10, 26

state variable, 26

tableau, 20, 104, 154, 161, 162,

178

calculus

internalized, 95

prefixed, 96

closed, 103

tableau calculus, 19

$\mathcal{T}_{\mathcal{H}_{\text {b-f }}}^{\mathrm{i}} 113$

$\mathcal{T}_{\mathcal{H}_{\text {b-f }}^{\mathbf{p}^{\text {b-f }}}}^{\mathrm{T}^{\prime}} 99,101$

$\mathcal{T}_{\mathcal{M}\left(\mathrm{E}_{n}\right)}^{i}, 149$

$\mathcal{T}_{\mathcal{M}\left(\mathrm{E}_{n}\right)}^{P}, 137$

$\mathcal{T}_{\mathcal{S H O Q}}, 155$

analytical, 20

constructive, 20, 170, 182

cumulative, 154

internalized, 20, 112, 149

prefixed, 20, 99, 137

tableau rule

auxiliary, 19

branching, 20

clash, 19

decomposition, 19

deterministic, 20

generating, 172

shrinking, 172

Tarskian semantics, 26

tense logic

fourth grade, 37

third grade, 37 
Index 215

termination, 20, 121, 125, 142, 145, 158, 173, 189

tile-type, 59

tiling, 59, 64, 71

Tinchev, T., 37

Tishkovsky, D., 4, 110

Tobies, S., 84

Truth Lemma, 56

Turing Machine polynomially space bounded, 14

polynomially time bounded, 14

twins, 131

two-dimensional binary counter, 62

type, 153

Tzakova, M., 2, 38, 95, 127

unravelling, 175

urfather

identity, 131

inclusion, 129

nominal, 128

quasi-, 131

validity problem, 12

variable

path, 24, 37

Venema, Y., 9

Wolter, F., 197

Zakharyaschev, M., 197 
REVIEWER

Marek Nasieniewski

PROOFREADING

Mark Tardi

TYPESETTING

Michat Zawidzki

ATEX style classthesis, (c) copyright by André Miede

COVER DESIGN

Magdalena Papiernik-Korczak

The book "Deductive systems and the decidability problem for hybrid logics" is a part of the project financed from the funds of the National Science Centre of Poland awarded by the decision number DEC-2011/01/N/HS1/01979

Printed directly from camera-ready materials provided to Łódź University Press by the Department of Logic and Methodology of Sciences

PRINT AND SETTING

Quick Druk 